\title{
Mapping the primate brain with network analysis
}

\author{
Citation for published version (APA):
}

Goulas, A. (2013). Mapping the primate brain with network analysis. [Doctoral Thesis, Maastricht University]. Maastricht University. https://doi.org/10.26481/dis.20130419ag

Document status and date:

Published: 01/01/2013

DOI:

10.26481/dis.20130419ag

Document Version:

Publisher's PDF, also known as Version of record

\section{Please check the document version of this publication:}

- A submitted manuscript is the version of the article upon submission and before peer-review. There can be important differences between the submitted version and the official published version of record.

People interested in the research are advised to contact the author for the final version of the publication, or visit the DOI to the publisher's website.

- The final author version and the galley proof are versions of the publication after peer review.

- The final published version features the final layout of the paper including the volume, issue and page numbers.

Link to publication

\footnotetext{
General rights rights.

- You may freely distribute the URL identifying the publication in the public portal. please follow below link for the End User Agreement:

www.umlib.nl/taverne-license

Take down policy

If you believe that this document breaches copyright please contact us at:

repository@maastrichtuniversity.nl

providing details and we will investigate your claim.
}

Copyright and moral rights for the publications made accessible in the public portal are retained by the authors and/or other copyright owners and it is a condition of accessing publications that users recognise and abide by the legal requirements associated with these

- Users may download and print one copy of any publication from the public portal for the purpose of private study or research.

- You may not further distribute the material or use it for any profit-making activity or commercial gain

If the publication is distributed under the terms of Article $25 \mathrm{fa}$ of the Dutch Copyright Act, indicated by the "Taverne" license above, 


\section{Mapping the primate brain with network analysis}

\section{Alexandros Goulas}





\section{Mapping the primate brain with network analysis}

Ter verkrijging van de graad van doctor aan de Universiteit Maastricht, op gezag van de Rector Magnificus, Prof. Dr. L.L.G. Soete volgens het besluit van het College van Decanen,

in het openbaar te verdedigen

op vrijdag 19 april 2013 om 12:00 uur

door

Alexandros Goulas

geboren op 3 mei 1982 te Athene, Griekenland 


\section{Promotor:}

prof. dr. Elia Formisano

prof. dr. Harry BM Uylings (VU Medical Center)

\section{Co-promotor:}

dr. Peter Stiers

\section{Reading committee:}

prof. dr. Rainer Goebel (chair)

prof. dr. Hilleke Hulshoff Pol (UMC Utrecht)

dr. Alard Roebroeck

dr. Kâmil Uludağ 


\section{Contents}

Chapter 1

Introduction

Chapter 2

Unravelling the intrinsic functional organization of the human lateral frontal cortex: a parcellation scheme based on resting-state fMRI

Chapter 3

Mapping the hierarchical layout of the structural network of the macaque prefrontal cortex

Chapter 4

Quantitative comparative analysis of structural connectivity patterns in the macaque and human brain

Chapter 5

Maturation of task-induced brain activation and long range functional connectivity in adolescence revealed by multivariate pattern classification

Chapter 6

Discussion

Samenvatting 

Chapter 1

Introduction 


\section{Early views on the architecture of the cerebral cortex: cortical fields and connectivity}

The brain constitutes the substrate of our mental capacities and it has been identified as such at least as early as the late Middle Age and early Renaissance (Finger, 2000).

Groundbreaking studies constituting the more immediate precursors of modern neuroscience at the end of the $19^{\text {th }}$ and beginning of the $20^{\text {th }}$ century have unravelled the complex architecture of the brain of humans and animals, suggesting that the cortical sheet is a mosaic of distinct cortical fields (Campbell, 1905; Vogt, 1910; Brodmann, 1909; von Economo and Koskinas, 1925) (see Fig. 1). Such cortical fields, i.e. distinct subregions, can be differentiated through microstructural criteria. Criteria commonly used are cyto-architecture, i.e. the organization of cells across the layers of the cerebral cortex, and myelo-architecture, i.e. the myelination of nerve fibers across the layers of the cerebral cortex. Additionally, recepto-architecture, i.e. the distribution across the cortical sheet of specific receptors that constitute part of the molecular underpinnings of brain functioning, is also used for delineating cortical fields (Zilles et al., 2002). These cortical fields can be conceived as the basic "organs of the cerebral cortex" (Brodmann, 1909).
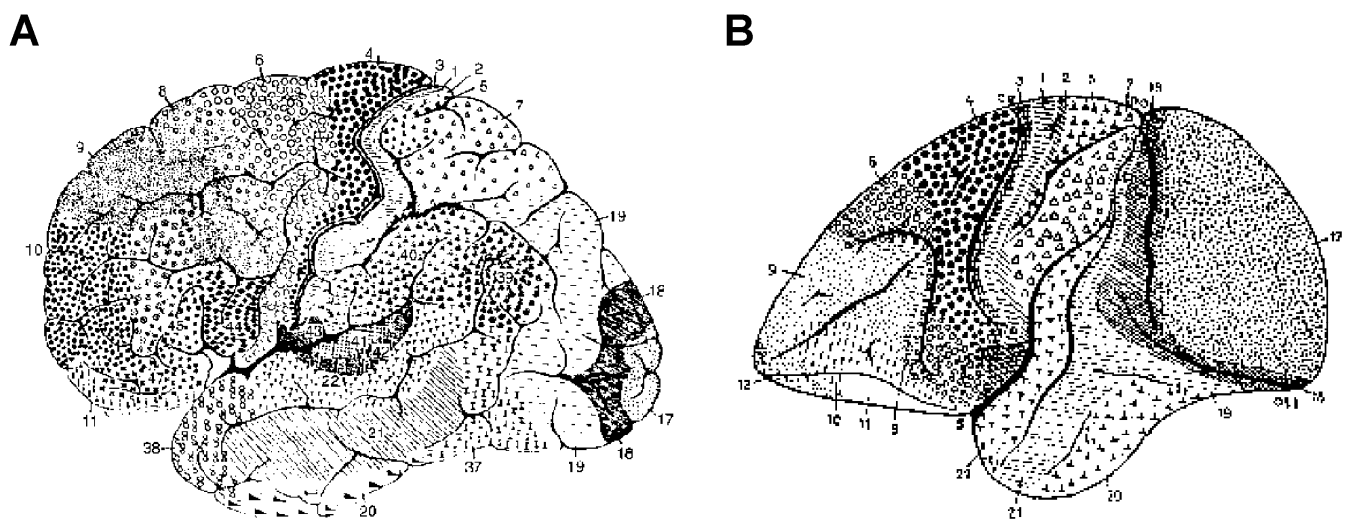

Figure 1. Maps depicting distinct cortical fields differentiated through their cytoarchitectonic features. Cytoarchitectonic maps depict the A. human (Brodmann, 1909) and B. monkey (cercopithicus) (Brodmann, 1905) cerebral cortex.

These basic organs/units of the brain exhibit a complex intrinsic and extrinsic connectivity pattern. The later type of connectivity refers to long distance connections/fibers between the distinct subregions of the brain. These fibers constitute the main part of the white matter. The white matter has been conceived as highly orderly and structured as early as the $17^{\text {th }}$ century, as reflected in the observations of Nicolaus Steno. In the late $19^{\text {th }}$ century observations unravelled orderly fiber bundles, like the cingulum bundle. Such observations started to unravel the fiber pathways of the cerebral cortex. The discovery that these fiber bundles are actually myelinated axons originating from nerve cells located at the grey matter established them as the anatomical substrate of long distance communication of brain regions (Schmahmann and Pandya, 2007) (see Fig 2). 


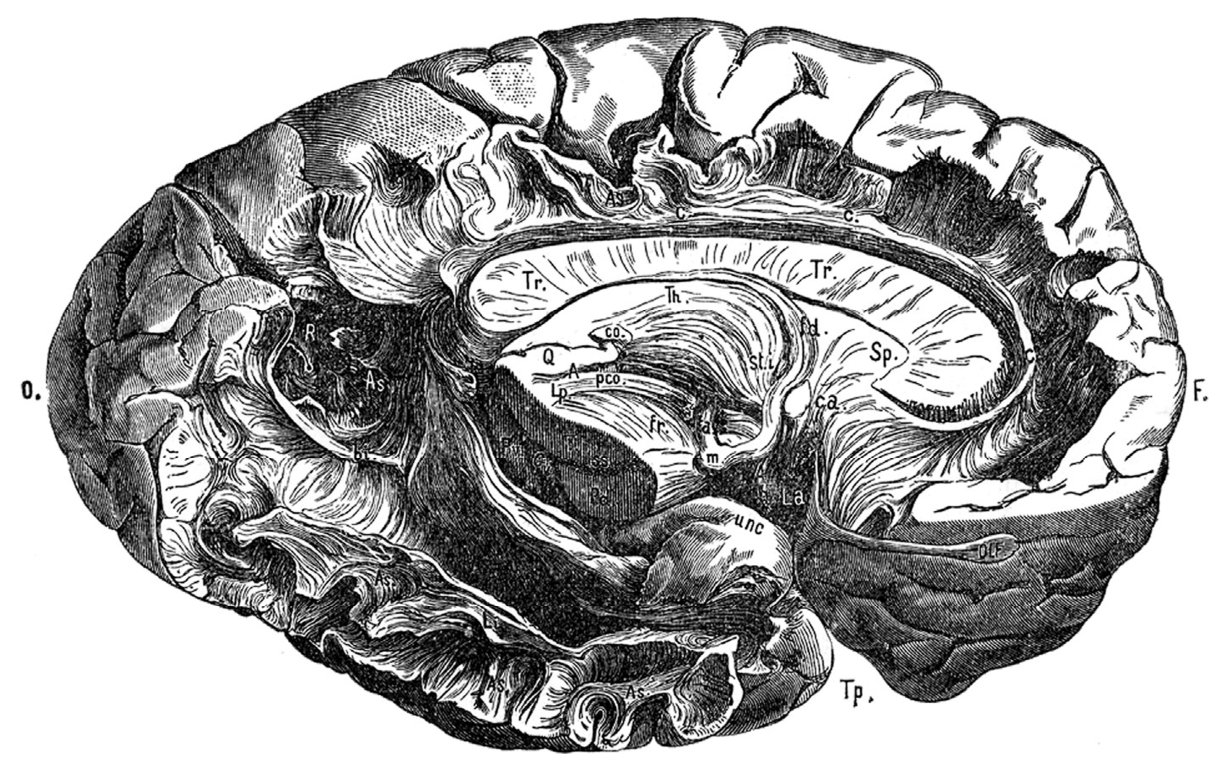

Figure 2. Medial view of the human cerebral cortex. Various fiber bundles linking the distinct brain regions are visible, e.g. the cingulum bundle (labelled "C"). Figure published originally in Meynert, 1885.

Early observations highlight the importance of the connections of a region to the function performed by that region. The following passage is illustrative: “...to begin with, my own observations have definitely proved that immediately investing the visuosensory area there exists a moderately extensive field of cortex, possessing a specialized type of arrangement of the nerve cells and nerve fibres, entirely different both from that in the visuo-sensory area and that in the more outlying parts: and granted that the calcarine area is solely devoted to the reception of primary visual stimuli, the mere existence of a second area placed in such immediate contiguity suggests the likelihood that it is concerned with the sorting out and further elaboration of these stimuli. More than this, the arrangement of the fibers in this investing area suggests that they carry corticifugal instead of corticipetal impressions, that they are the fibres, in other words, which combine to form the strands joining the visual with other centres and helping to make the visual function so complex." (Campbell, 1905, p. 146). Such observations and conceptualizations establish the ground of structure-function dependencies and highlighting the importance of brain connectivity for fundamental and clinical research (Ffytche and Catani, 2005).

The aforementioned early findings conjointly indicate that the brain can be conceived as a complex system, i.e. a network. In this network the distinct subregions constitute the nodes of the network while the long distance fiber bundles constitute its connections. Despite the powerful insights provided from early investigations, many of them were characterized by limitations such as lack of observer-independent techniques, quantification, storing and availability of data, invasive nature of the techniques used, and lack of computational and analytic tools. Hence, various aspects of early investigations fall short of the challenges posed by a realistic, fine grained examination of such a complex structure as the cerebral cortex. 


\section{Modern neuroscience, the re-emergence of old theories and the development of new views}

In recent years key advancements in the field of neuroscience offered the opportunity to tackle older problems and face new challenges with a fresh perspective. These key advancements are:

1. New in vivo brain imaging methods, i.e. functional Magnetic Resonance Imaging (fMRI) and diffusion weighted imaging (DWI) (e.g. Huettel et al., 2004; Johansen-Berg and Behrens, 2009).

2. Analytic tools, usually borrowed from other scientific disciplines, and increased computational resources (e.g. Rubinov and Sporns, 2010; Formisano et al., 2008).

3. Data storage and sharing through the implementation of neuroinformatics databases (e.g. Akil et al., 2011; Kötter, 2004).

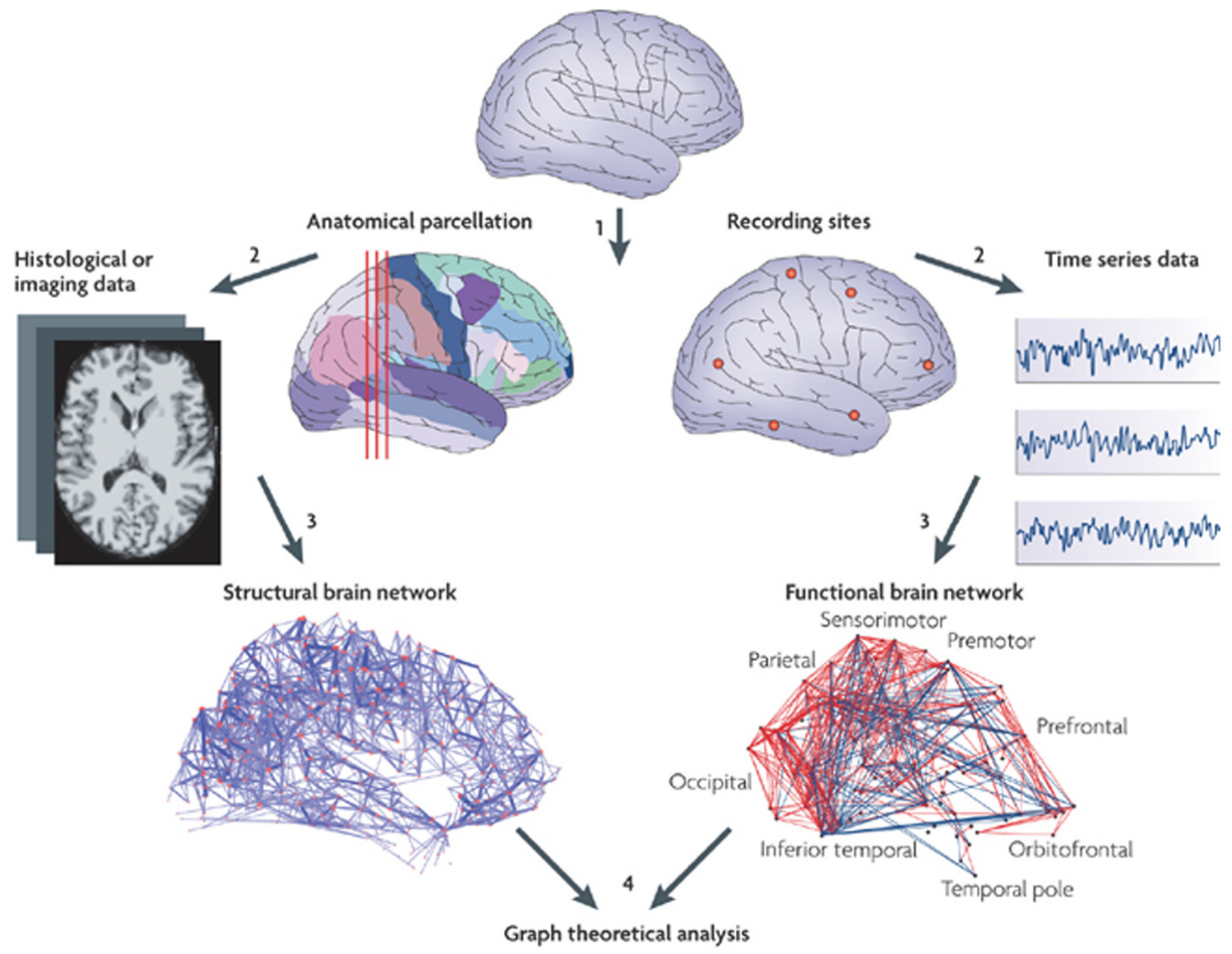

Figure 3. Construction of the human connectome by measuring structural and functional connectivity. The figure depicts the necessary steps to be taken for such a construction. The brain of a subject can be parcellated, e.g. based on an atlas and subsequently the inter-regional connections can be traced with DWI (steps 2 and 3 in the left analysis stream). In a similar fashion brain activity measured e.g. with fMRI is recorder from certain brain regions or from the whole brain and the computation of the functional connectivity between the time series of these recording sites results in the construction of the functional connectome (steps 2 and 3 in the right analysis stream). The functional and structural connectomes can be subsequently analyzed with tools from network science. Figure originally published in Bullmore and Sporns, 2009. 
Such advancements rendered possible the re-emergence of older ideas to the forefront. For instance, the conceptualization of the brain as a complex network and the subsequent effort of collating and mapping connections of the brain of different species at multiple levels resulted in the field refered to as connectome research (Sporns et al., 2005) (see Fig. 3). Assembling the connectome of brains of humans and animals with the boost offered by the aforementioned key advancements, new avenues were opened for fundamental, comparative and clinical studies. Moreover, the "network view" towards the brain is also strengthened by the discovery of large scale functional networks. Such networks can be detected in task-independent intrinsic neuronal activation measured during "rest". Hence, such networks are commonly known as resting state networks (De Luca et al., 2006) and reflect the intrinsic functional architecture of the brain. Hence, two types of connectivity can be discerned and will be used for our research purposes. First, structural connectivity between two regions refers to the existence of a fiber bundle that connects them. Secondly, functional connectivity refers to the temporal dependence of the neural activation of two regions, e.g. as measured with fMRI. Both types of networks can be analyzed with tools from network science (see Fig. 3).

The value of the network approach in order to study the brain is reflected in recent studies that have unravelled basic properties of the architecture of the brain, such as its small world structure and the presence of a rich club organization (Sporns and Zwi, 2004; van den Heuvel and Sporns, 2011). Additionally, in vivo delineation of distinct subregions was rendered possible with the aid of both structural (Johansen-Berg et al., 2004) and functional (Cohen et al., 2008) connectivity. Topological changes at the whole brain large scale level were found to be associated with mental disorders (van den Heuvel et al., 2010). Furthermore, interspecies comparisons of brain connectivity started to provide a valuable link between human and macaque research (Croxson et al., 2005), comprehensive maps of the large scale connectivity of the macaque brain started to be assembled (Modha and Singh, 2010), and structure-function dependency was demonstrated (Kötter and Sommer, 2000; Passingham et al., 2002). Moreover, data clustering techniques, exploiting the wealth of data gathered in neuroinformatics databases, revealed that each distinct brain region has a unique connectivity. Hence, the connectivity of a region can be conceived as its "connectivity fingerprint", which in turn is linked with its "functional fingerprint". Consequently, the connectivity of a region can constrain what it "can do" (Passingham et al., 2002). Such findings led to a reappraisal of large scale connectivity and its importance to brain function. Consequently, it has become clear that large scale brain connectivity can be used as a guide to cognition (Bressler and Menon, 2010).

\section{Correspondance between structure and function in the brain}

In order to illustrate the interdependency of connectivity and function, we will briefly discuss studies, which by exploiting the key advancements previously mentioned, offer a quantitative assessment of structure-function correspondence.

Based on a collation of data describing the connectivity of the cat brain (Scannell and Young, 1993; Scannell et al., 1995), and with the aid of network analysis tools, it has been demonstrated that the cat cortex can be partitioned in 4 "modules" which correspond to distinct anatomical structures with relative functional specializations. Regions belonging to the same module exhibit higher degree of similarity of their whole 
brain connectivity patterns when compared to regions belonging to different modules (Zamora- López et al., 2010).

A distinct class of studies have addressed structure-function correspondences by examining more systematically the relation of the large scale functional connectivity and the underlying structural connectivity. Computational modelling using realistic neuroanatomical connections of the macaque brain highlights the very good correspondance of structural and functional connectivity, especially evident when neuronal interactions occur in time-scales comparable to the ones measured with fMRI during "rest" (Honey et al., 2007). Moreover, empirical studies demonstrate that the large scale functional networks are structurally linked (van den Heuvel et al., 2009) and that functional connectivity between structurally unconnected regions can be explained by the patterns of efferents of the involved regions (Adachi et al., 2011).

Exploiting DWI for the in vivo mapping of the structural connections of the human brain, Johansen-Berg et al. 2004 parcellated the medial wall in two distinct regions. This parcellation was based on the abrupt change of the whole brain connectivity profiles of these regions. Such differences were detectable by a clustering algorithm. This study constitutes one of the first examples of the feasibility of in vivo connectivity based parcellation of brain regions. The resulting regions not only corresponded to known anatomical regions, i.e. SMA and pre-SMA, but also exhibited different functional profiles. While SMA appeared motor sensitive, the pre-SMA had a "cognitive" functional profile since it was active in a serial subtraction task. Hence, regions that could be delineated by exploiting transitions of their large scale connectivity profiles, also exhibit distinct functional/cognitive signatures.

A recent elegant study provides further evidence for the structure-function correspondance. By using machine learning algorithms for regression, the connectivity of the fusiform gyrus with the rest of brain, as assessed with DWI, appeared to be an excellent predictor of the fusiform gyrus activation during observation of faces (Saygin et al., 2012).

The above computational and empirical studies in the human and non-human brain demonstrate that structural connectivity and functional connectivity are closely linked, connectivity constraints function, and distinct anatomical regions exhibit distinct connectivity profiles. Such properties can be used for the in vivo connectivity based parcellation of the brain and the elucidation of their role in cognition.

\section{Challenges and open issues: in vivo cartography and connectivity}

Despite methodological advancements and the accumulated novel findings, there are striking gaps in our knowledge about the architecture and function of the brain.

Currently there is no contemporary comprehensive map of the totality of the human cerebral cortex which can summarize its distinct regions based on cyto- myelo- and recepto-architecture, along with their distinctive connectivity and functional properties. Consequently, maps from early investigators of the previous century are still used, but such maps are not always compatible, are observer dependent and do not quantify interindividual variability (Uylings et al., 2005). Efforts during the last decade try to fill this gap through quantitative mapping of cortical areas and the creation of cytoarchitectonic probabilistic maps (Eickhoff et al., 2005; Rajkowska and Goldman-Rakic, 1995), receptor based mapping (Amunts et al., 2010), task based functional maps (Frost and Goebel, 2011), in vivo myelin based mapping (Glasser and Van Essen, 2011) and connectivity based mapping (Cohen et al., 2008). Such combined efforts will finally 
lead to a multimodal atlas which is necessary to describe the multilayered architecture of the brain (Toga et al., 2006). However, for large parts of the brain probabilistic maps of the distinct cortical fields are currently lacking. This is particularly evident for the frontal lobes (see Fig. 4). This fact combined with excessive intersubject variability makes problematic the inference of a cortical field in an individual brain. Consequently, there is the need for the mapping of the subregions of parts of the brain that are relatively unexplored, such as the frontal lobes, and the examination of their connectivity. Such mapping should also be performed in vivo, offering numerous possibilities for clinical applications, structure-function examination and neurosurgical pre-operative mapping.

Due to methodological and ethical reasons brain research in animals constitutes a major source of our knowledge on brain function. Electrophysiological, connectivity, and parcellation studies performed in the macaque brain that span several decades, offer a wealth of information, unparalleled in the human brain. Hence, the macaque brain functions as a model for the human brain (Passingham, 2009). Extrapolating findings from one species to the other can also introduce inaccuracies due to unique features of the human brain that emerged during primate evolution (Preuss, 2000).

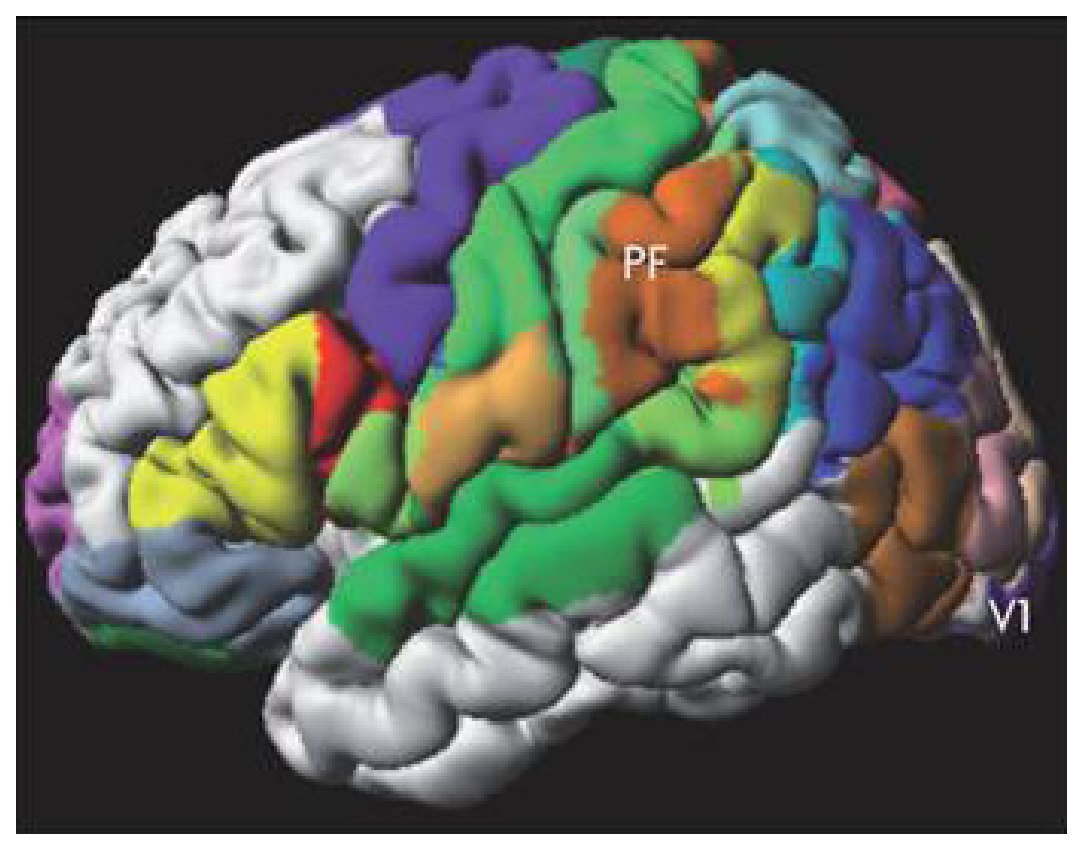

Figure 4. The Jülich-Düsseldorf atlas of cytoarchitectonically defined cortical fields (Eickhoff et al., 2005). Each colour denotes a distinct cortical field and the spatial extend corresponds to its maximum probability. Note the incompleteness of the atlas, especially in the prefrontal cortex.

Two major and interrelated problems can be discerned with respect to models based on the macaque. First, evidence in favour of specific models and suggested architectures of brain regions usually rely on descriptive approaches of qualitative data, without rigorous quantification of the assumed principles. This usually involves a restricted set of data involving a limited amount of studies. Second, extrapolations from the macaque to the human brain rely on assumptions of homology. In some cases, homology for a small subset of cortical regions is established on functional, topological and cytoarchitectonic 


\section{Chapter 1}

criteria (Fig. 5). However, establishment of homology, or absence thereof, does not always stem from a direct testing of the fulfilment of additional homology criteria like similarity of connectivity patterns (Uylings et al., 2003). Thus, there is the need for quantification of the fulfilment of such connectivity based homology criteria, especially in the light of evidence suggesting that connectivity constraints function. Such direct comparisons should focus on the connectivity of the brains of macaques and humans (Passingham, 2009; Croxson et al., 2005). Such investigations will help establish a "translational bridge" between macaque and human brain research by unravelling preservation of connectivity features, or absence thereof, during primate evolution.

The conceptualization of the brain as a complex network resulted in considerable progress for the understanding of large scale reconfigurations in connectivity during brain development (e.g. Supekar et al., 2009). These reconfigurations are also evident in the functional domain, since task based paradigms reveal age related differences in BOLD responses (e.g. Keulers et al., 2012). However, the majority of developmental studies rely on univariate statistical analysis which might be inappropriate for detecting subtle large scale differences across age groups. This also forces the choice of comparing age groups with considerably large age spans. Moreover, very few studies examine function and connectivity conjointly in order to uncover potential relations. Taken together the above illustrate the need for a more sophisticated approach allowing to track subtle developmental changes and thus investigate the development trajectory without the limitations posed by large spans, e.g. during adolescence. Such investigations should ideally encompass both the functional and connectivity domain.

The aforementioned open issues set the context for the research goals of the present thesis.

\section{Research goals of the thesis}

In Chapter 2 an in vivo parcellation scheme of the lateral frontal cortex of the human brain will be presented. This study aims at contributing to the growing efforts for the in vivo parcellation of the cortex. More specifically, the two main goals of the study are the following: What are the distinct subregions/cortical fields of the lateral frontal cortex that can be deciphered in vivo? What is the connectivity profile of these cortical fields? The results from these two main research goals have several important implications. The connectivity of each region can inform us about its functionality. The relation of the connectivity profiles of the distinct subregions constitute the intrinsic architecture of the lateral frontal cortex and can thus inform proposed models. The connectivity of the subregions of the proposed parcellation, which are assigned to putative anatomical substrates, can be associated with the connectivity of assumed homologues in the macaque. Hence, the parcellation scheme and connectivity of the lateral frontal cortex can be used as a link between human and macaque research. Additionally, the in vivo parcellation can pave the way for more accurate targeting of these regions for purposes like pre-operative imaging for neurosurgery. Lastly, the feasibility of an in vivo parcellation renders possible the examination of the functional properties of the delineated cortical fields, i.e. their responsiveness to various tasks tapping into a diverge set of cognitive domains.

As it is already mentioned, the findings of the previous chapter can also inform popular models of the frontal cortex. However, the study described in the previous chapter was not specifically designed for addressing specific models. In Chapter 3 we will specifically investigate the principles and predictions of the so called anterior- 
posterior hierarchical model, as articulated in the context of the structural connectivity of the macaque prefrontal cortex. In doing so, we will adopt an approach that overcomes the problems illustrated in previous paragraphs, i.e. lack of quantification, usage of a limited set of data. More specifically, we will use data from the neuroinformatics database CoCoMac (http://cocomac.g-node.org/). This database contains information about the connectivity of the macaque brain by collating findings from numerous tracing studies. Hence, the data used for the study presented in Chapter 3 constitute a best estimate of macaque prefrontal connectivity, representing a manifold of studies spanning several decades. Additionally, for assessing the principles and predictions of the model, formal tools from network science will be employed. This approach will allow us to establish if the anterior-posterior hierarchical model is tenable and further gain insight into the architecture of the macaque prefrontal cortex.

In the aforementioned chapters certain links between human and macaque research are visible. However, in both cases data are obtained from either humans (Chapter 2) or macaques (Chapter 3) and any relation between the species is established with the aid of findings from the literature. In Chapter 4 we will perform a direct interspecies comparison. To this end, we assemble the connectome of each species. For humans we employ diffusion imaging and for macaques data collated in the CoCoMac database. We perform quantitative analysis of the whole brain connectivity of the two species. Chapter 4 describes key findings such as the embedding of the various brain regions in the two species, the preservation of their connectivity patterns and global topological features like the rich club organization of both connectomes. Through rigid quantitative analysis of the connectomes of the two species, a research line that could be named comparative connectomics, novel findings on the structural organization during primate evolution are brought forth. Such findings are valuable for building a translational bridge between human and macaque research.

In Chapter 5 we approach connectivity of the human brain from a developmental point of view. We study the maturation of short and long range functional connections during adolescence. Moreover, apart from connectivity, we examine task induced activation patterns. The study involves age groups with a minimal age difference $(13,17$ and 21 years). In that way we can investigate maturation changes in adolescence without being forced to adopt huge age spans. Such differences are subtle and hence appropriate analytic tools are needed. To this end we use multivariate machine learning techniques to decipher age specific connectivity and task related discriminative patterns. Conjointly the above approach permits tracing and interrelating subtle connectivity and task related changes in adolescence. Chapter 6 constitutes the Discussion section of the present thesis. 


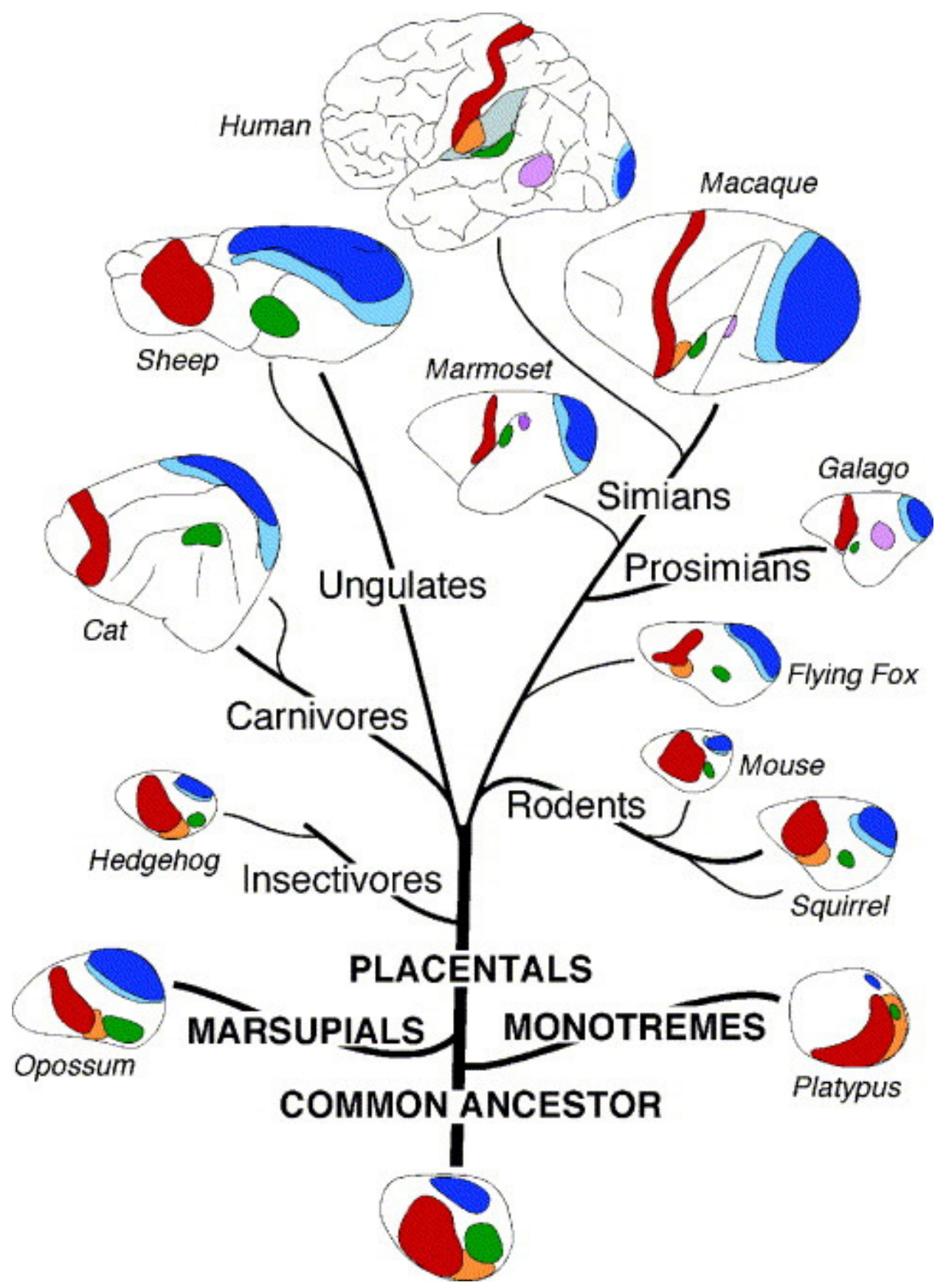

Figure 5. Evolutionary tree of the mammalian brain. Different colors delineate cortical fields that are considered homologous. Homology is inferred from anatomical and functional features. Cortical fields represent basic sensory fields: visual (V1 dark blue V2 light blue), auditory (A1 green), and somatosensory (S1 red S2 orange). Figure originally published in Krubitzer and Kahn, 2003.

\section{$\underline{\text { Modalities and methods }}$}

The discovery of the Blood Oxygen Level Dependent (BOLD) contrast gave birth to the fMRI (Huettel et al., 2004). fMRI allows the in vivo examination of the totality of the brain with very good spatial resolution and a temporal resolution allowing the investigation of numerous neuroscientific questions. The BOLD signal is an indirect 
measurement of neuronal activity. It relies on the relation of cerebral blood flow, cerebral blood volume and the rate of metabolism of oxygen, all of which constitute a cascade of physiological responses accompanying neuronal activity (Huettel et al., 2004). The BOLD signal correlates with underlying neuronal activity (Logothetis et al., 2001) and its exact neurovascular mechanisms are currently under investigation (Goense et al., 2012). fMRI has been extensively used in order to examine cognitive mechanisms. Additionally, the discovery of large scale functional networks, detected at low fluctuations of the BOLD signal while the subject is not performing an explicit task - thus at "rest" - established resting state fMRI as a popular tool for studying the functional connectivity and architecture of the brain. For the aforementioned reasons we chose fMRI as the modality appropriate for addressing certain research goals of this thesis.

In order to study the structural connections of the brain techniques like DWI can be used. DWI relies on the diffusion of water molecules in brain tissue which is detectable with an MR apparatus. The diffusion is constrained by the geometrical structure of the brain tissue, i.e. in the current context, the fiber bundles (Johansen-Berg and Behrens, 2009). Various models can be applied to assess intra-voxel diffusion direction and several tractography methods can be used for unravelling the fiber bundles that constitute the structural connectivity architecture of the brain (Bastiani et al., 2012). DWI has been used for constructing the human connectome, comparing connections of the human and the macaque brain, as well as in clinical contexts (Johansen-Berg and Behrens, 2009). Such features render DWI suitable for fulfilling some of the research goals of the present thesis.

Modern neuroscience is equipped with neuroinformatics databases that allow the storage, handling, and extraction of meaningful information from large datasets. One such database is CoCoMac which consists of collations of results from tracing studies performed in the macaque brain. The hundreds of studies collated span several decades and thus the information stored in CoCoMac represent the most complete set of data on connectivity of the macaque. The massive amount of information involved led to the development of tailored algebra and algorithms for the exploitation of the wealth of this information (Kötter, 2004; Stephan et al., 2000). Hence, datasets assembled from the CoCoMac database reflect knowledge accumulated over several decades and renders them valuable for studying the connectional architecture of the macaque brain as well as for inter-species comparisons. Consequently, we will use such datasets for addressing research goals of this thesis.

If the brain can be conceived as a complex network then it must also be treated as one. In other words, there is the need for appropriate tools for studying this network. Such tools are mostly borrowed from other scientific disciplines like computational/systems biology and network science. Such disciplines have also faced the same class of problems that neuroscientists now face, namely understanding complex systems, a procedure involving the analysis of a vast amount of data. Consequently, tools were developed for appropriately addressing such issues. In the context of neuroscience such tools have been successfully adopted in both the human (Hagmann et al., 2008) and the macaque brain (Modha and Singh, 2010). We will follow the same rationale and also borrow tools from network science in order to address the research questions of this thesis. 


\section{Chapter 1}

\section{$\underline{\text { References }}$}

Adachi Y, Osada T, Sporns O, Watanabe T, Matsui T, Miyamoto K, Miyashita Y (2011) Functional connectivity between anatomically unconnected areas is shaped by collective network-level effects in the macaque cortex. Cereb Cortex 22: 1586-1592.

Akil H, Martone ME, Van Essen D (2011) Challenges and Opportunities in Mining Neuroscience Data. Science 331:708-711.

Amunts K, Lenzen M, Friederici AD, Schleicher A, Morosan P, Palomero-Gallagher N, Zilles K (2010) Broca's region: novel organizational principles and multiple receptor mapping. PLoS Biol 8:e1000489.

Bastiani M, Shah NJ, Goebel R, Roebroeck A (2012) Human cortical connectome reconstruction from diffusion weighted MRI: The effect of tractography algorithm. NeuroImage 62:1732-1749.

Bressler SL, Menon V (2010) Large-scale brain networks in cognition: emerging methods and principles. Trends Cog Sci 14:277-290.

Brodmann K (1905) Beiträge zur histologischen Lokalisation der Grosshirnrinde: dritte Mitteilung: Die Rindenfelder der niederen Affen. Journal für Psychologie und Neurologie 4: 177-226.

Brodmann, K (1909) Vergleichende Localisationslehre der Grosshirnrinde in ihren Prinzipien dargestellt auf Grund des Zellenbaues. Leipzig: Barth.

Bullmore E, Sporns O (2009) Complex brain networks: graph theoretical analysis of structural and functional systems. Nat Rev Neurosci 10:186-98.

Campbell, AW (1905) Histological studies on the localisation of cerebral function. Cambridge University Press.

Cohen AL, Fair D, Dosenbach NUF, Miezin FM, Dierker D, Van Essen DC, Schlaggar BL, Petersen SE (2008) Defining functional areas in individual human brains using resting functional connectivity MRI. NeuroImage 41:45-57.

Croxson PL, Johansen-Berg H, Behrens TEJ, Robson MD, Pinsk M, Gross CG, Richter W, Richter MC, Kastner S, Rushworth MFS (2005) Quantitative investigation of connections of the prefrontal cortex in the human and macaque using probabilistic diffusion tractography. J Neurosci 25:8854-66.

De Luca M, Beckmann CF, De Stefano N, Matthews PM, Smith SM (2006) fMRI resting state networks define distinct modes of long-distance interactions in the human brain. NeuroImage 29:1359-67.

Eickhoff SB, Stephan KE, Mohlberg H, Grefkes C, Fink GR, Amunts K, Zilles K (2005) A new SPM toolbox for combining probabilistic cytoarchitectonic maps and functional imaging data. NeuroImage 25:1325 - 1335.

Ffytche DH, Catani M (2005) Beyond localization: from hodology to function. Philos Trans R Soc Lond B Biol Sci 360:767-779.

Finger S (2000) Minds behind the brain: a history of the pioneers and their discoveries. New York: Oxford University Press.

Formisano E, De Martino F, Valente G (2008) Multivariate analysis of fMRI time series: classification and regression of brain responses using machine learning. Magn Reson Imaging 26:921-934.

Frost MA, Goebel R (2012) Measuring structural - functional correspondence: Spatial variability of specialised brain regions after macro-anatomical alignment. NeuroImage 59:1369-1381.

Goense J, Merkle H, Logothetis NK (2012) High-resolution fMRI reveals laminar differences in neurovascular coupling between positive and negative BOLD responses. Neuron 76:629-639.

Glasser MF, Van Essen DC (2011) Mapping human cortical areas in vivo based on myelin content as revealed by T1- and T2-weighted MRI. J Neurosci 31:11597-11616. 


\section{Chapter 1}

Hagmann P, Cammoun L, Gigandet X, Meuli R, Honey CJ, Wedeen VJ, Sporns O (2008) Mapping the structural core of human cerebral cortex. PLoS Biol 6:e159.

Honey CJ, Sporns O, Cammoun L, Gigandet X, Thiran JP, Meuli R, Hagmann P (2009) Predicting human resting-state functional connectivity from structural connectivity. Proc Natl Acad Sci U S A 106:2035-40.

Huettel SA, Song AW, McCarthy G (2004) Functional Magnetic Resonance Imaging, Sinauer Associates Inc, Publishers Sunderland, Massachusetts, USA.

Johansen-Berg H, Behrens TEJ (eds) (2009) Diffusion MRI: From quantitative measurement to in-vivo neuroanatomy. Academic Press.

Johansen-Berg H, Behrens TEJ, Robson MD, Drobnjak I, Rushworth MFS, Brady JM, Smith SM, Higham DJ, Matthews PM (2004) Changes in connectivity profiles define functionally distinct regions in human medial frontal cortex. Proc Natl Acad Sci U S A 101:13335-40.

Keulers EHH, Goulas A, Jolles J, Stiers P (2012) Maturation of task-induced brain activation and long range functional connectivity in adolescence revealed by multivariate pattern classification. NeuroImage 60:12501265 .

Kötter R, Sommer FT (2000) Global relationship between anatomical connectivity and activity propagation in the cerebral cortex. Philos Trans R Soc Lond B Biol Sci 355:127-34.

Kötter R. (2004) Online retrieval, processing, and visualization of primate connectivity data from the CoCoMac database. Neuroinformatics 2:127:144.

Krubitzer L, Kahn DM (2003) Nature versus nurture revisited: an old idea with a new twist. Prog Neurobiol $70: 33-52$.

Logothetis NK, Pauls J, Augath M, Trinath T, Oeltermann A (2001) Neurophysiological investigation of the basis of the fMRI signal. Nature 412:150-7.

Meynert T (1885) Psychiatry. A clinical treatise on diseases of the forebrain based upon its structure, function and nutrition. Putnam's Sons, London.

Modha DS, Singh R (2010) Network architecture of the long-distance pathways in the macaque brain. Proc Natl Acad Sci U S A 107:13485-90.

Passingham RE, Stephan KE, Kötter R (2002) The anatomical basis of functional localization in the cortex. Nat Rev Neurosci 3:606-16.

Passingham R (2009) How good is the macaque monkey model of the human brain? Curr Opin Neurobiol 19:6-11.

Preuss TM (2000) Taking the measure of diversity: Comparative alternatives to the model-animal paradigm in cortical neuroscience. Brain Behav Evol 55:287-299.

Rajkowska G, Goldman-Rakic PS (1995) Cytoarchitectonic definition of prefrontal areas in the normal human cortex: II Variability in locations of areas 9 and 46 and relationship to the Tailarach coordinate system. Cereb Cortex 5:323-337.

Rubinov M, Sporns O (2010) Complex network measures of brain connectivity: uses and interpretations. NeuroImage 52:1059-69.

Saygin ZM, Osher DE, Koldewyn K, Reynolds G, Gabrieli JDE, Saxe RR (2011) Anatomical connectivity patterns predict face selectivity in the fusiform gyrus. Nat Neurosci 15:321-327.

Scannell, JW, Blakemore, C, Young, MP (1995) Analysis of connectivity in the cat cerebral cortex. J Neurosci 15:1463-1483. 


\section{Chapter 1}

Schmahmann JD, Pandya DN (2007) Cerebral white matter - historical evolution of facts and notions concerning the organization of the fiber pathways of the brain. J Hist Neurosci 16:237-67.

Sporns O, Zwi J (2004) The small world of the cerebral cortex. Neuroinformatics 2:145-162.

Sporns O, Tononi G, Kötter R (2005) The Human Connectome: a structural description of the human brain. PloS Comput Biol 1:e42.

Stephan KE, Zilles K, Kötter R (2000) Coordinate-independent mapping of structural and functional data by objective relational transformation (ORT). Philos Trans R Soc Lond B Biol Sci 355:37-54.

Supekar K, Musen M, Menon V (2009) Development of large-scale functional brain networks in children. PLoS Biol 7:e1000157.

Toga AW, Thompson PM, Mori S, Amunts K, Zilles K (2006) Towards multimodal atlases of the human brain. Nat Neurosci Rev 7:952-966.

Uylings HBM, Groenewegen HJ, Kolb B (2003) Do rats have a prefrontal cortex? Behav Brain Res 146:3-17.

Uylings HBM, Rajkowska G, Sanz-Arigita E, Amunts K, Zilles K (2005) Consequences of large interindividual variability for human brain atlases: converging macroscopical imaging and microscopical neuroanatomy. Anat and Embryol 210:423-31.

van den Heuvel MP, Mandl RCW, Kahn RS, Hulshoff Pol HE (2009) Functionally linked resting-state networks reflect the underlying structural connectivity architecture of the human brain. Human Brain Mapp 30:3127-41.

van den Heuvel MP, Mandl RCW, Stam CJ, Kahn RS, Hulshoff Pol HE (2010) Aberrant frontal and temporal complex network structure in schizophrenia: a graph theoretical analysis. J Neurosci 30:15915-26.

van den Heuvel MP, Sporns O (2011) Rich-club organization of the human connectome. J Neurosci 31:1577515786.

Vogt O (1910) Die myeloarchitektonische Felderung des Menschlichen Stirnhirns. J Psychol Neurol 15:221232.

Von Economo C, Koskinas GN (1925) Die Cytoarchitektonik der Hirnrinde des erwachsenen Menschen. Textband und Atlas. Wien, Springer.

Young P, Scannell JW (1993) The connectional organization cat cerebral of neural systems in the cortex. Curr Biol 3:191-200.

Zamora-López G, Zhou C, Kurths J (2010) Cortical hubs form a module for multisensory integration on top of the hierarchy of cortical networks. Front Neuroinform 4:1-12.

Zilles K, Palomero-Gallagher N, Grefkes C, Scheperjans F, Boy C, Amunts K, Schleicher A, (2002) Architectonics of the human cerebral cortex and transmitter receptor fingerprints: reconciling functional neuroanatomy and neurochemistry. Eur Neuropsychopharmacol 12:587-599. 
Chapter 2

\section{Unravelling the intrinsic functional organization of the human lateral frontal cortex: a parcellation scheme based on resting-state fMRI}

Goulas A, Uylings HBM, Stiers P (2012) Unravelling the intrinsic functional organization of the human lateral frontal cortex: a parcellation scheme based on resting state fMRI. J Neurosci 32:10238 -10252. 


\begin{abstract}
Human and non-human primates exhibit flexible behaviour. Functional, anatomical and lesion studies indicate that the lateral frontal cortex (LFC) plays a pivotal role in such behaviour. LFC consists of distinct subregions exhibiting distinct connectivity patterns that possibly relate to functional specializations. Inference about the border of each subregion in the human brain is performed with the aid of macroscopic landmarks and/or cytoarchitectonic parcellations extrapolated in a stereotaxic system. However, the high inter-individual variability, the limited availability of cytoarchitectonic probabilistic maps and the absence of robust functional localizers, renders the in vivo delineation and examination of the LFC subregions challenging. In this study we use resting state fMRI (rsfMRI) for the in vivo parcellation of the human LFC, on a subjectwise and data-driven manner. This approach succeeds in uncovering neuroanatomically realistic subregions, with potential anatomical substrates including BA 46, 44, 45, 9 and related (sub)divisions. Ventral LFC subregions exhibit different functional connectivity (FC) which can account for different contributions in the language domain, while more dorsal adjacent subregions marked a transition to visuospatial/sensorymotor networks. Dorsal LFC subregions participate in known large-scale networks obeying an external/internal information processing dichotomy. Furthermore, we traced "families" of LFC subregions organized along the dorsal-ventral and anterior-posterior axis with distinct functional networks also encompassing specialized cingulate divisions. Similarities with the connectivity of macaque candidate homologues were observed, like the premotor affiliation of presumed BA 46. The current findings partially support dominant LFC models.
\end{abstract}




\section{$\underline{\text { Introduction }}$}

Human and non-human primates are characterized by flexible behaviour. Processes such as learning, integration of information and rule following, are essential for the successful interaction with the environment and accomplishment of everyday tasks. The frontal lobe has been identified as the brain structure that plays a pivotal role in these "higher order" processes (Goldman-Rakic, 1996; Miller and Cohen, 2001). Especially the LFC is implicated in diverse aspects of task execution (e.g. Koechlin et al., 2003; Brass et al., 2005; Stiers et al., 2010).

The LFC, like the rest of the cortex, is far from homogenous. Several LFC cytoarchitectonic parcellation schemes have appeared for the human brain, with variations in the number and extent of LFC divisions (Brodmann, 1909; Sarkissov et al., 1955; Petrides and Pandya, 1994) (Fig. 1). Moreover, tracing studies in the monkey and rodent frontal cortex have demonstrated that regions differentiated through their cytoarchitecture, also exhibit different connectivity patterns (e.g. Yeterian et al., 2012; Uylings et al., 2003). This "connectivity fingerprint" of each region seems to reflect a functional specialization, in line with evidence from lesion studies (Passingham et al., 2002; Petrides, 2005). The proper function and extensive repertoire of the LFC appears to rely on the interaction of these specialized regions (Wilson et al., 2010; Miller, 2000). Consequently their delineation and the further characterization of their connectivity properties are crucial for the better understanding of the LFC.

Several approaches have been adopted for the delineation of distinct regions in the human brain non-invasively. One approach uses macroscopic landmarks as predictors of the extent of a region but such an approach seems problematic for frontal regions, since discrepancies between the actual and predicted extent are observed (Fischl et al., 2008). Another common approach uses crisp cytoarchitectonic parcellation schemes, e.g. Brodmann's map, extrapolated in a standard stereotaxic system. However, such approaches seem inadequate since excessive interindividual variability has been demonstrated with respect to the exact location, shape and size of cytoarchitectonically defined regions (Uylings et al., 2005). Probabilistic maps have been introduced in order to express interindividual variability, but such maps are available only for a handful of (pre)frontal regions, namely regions 44 and 45 (Amunts et al., 1999) and regions 9 and 46 (Rajkowska and Goldman-Rakic, 1995).

Thus, it remains challenging to delineate and examine in vivo the distinct subregions of the LFC, especially in the absence of robust functional localizers. In the current study we capitalize on findings that demonstrate that rsfMRI can be used in order to functionally parcellate the cortex (Cohen et al., 2008; Shen et al., 2010). We employ a data-driven approach that unveils distinct subregions within the LFC on an individual basis, which seem to correspond well with subregions identified in cytoarchitectonic studies. Moreover, the whole-brain FC of these subregions could segregate them into "families". Additional analyses of neighbouring subregions located at the ventral/dorsal LFC elucidate the distinct large-scale functional networks that can relate to functional specializations.

\section{Materials and Methods}

Participants and data collection Twelve healthy right-handed participants were scanned for this study (8 females: mean age 22.5 years SD 2.4 year). Data were collected on a Siemens MAGNETOM Allegra 3T MRI head-only scanner. Head motion 
was constrained by the use of foam padding. A total number of 32 axial slices covering the whole-brain including the cerebellum were acquired by using a $\mathrm{T} 2 *$-weighted gradient echo planner pulse sequence $(\mathrm{TR}=2000 \mathrm{~ms}, \mathrm{TE}=30 \mathrm{~ms}, \mathrm{FOV}=224$, slice thickness $=4 \mathrm{~mm}$, matrix size $=64 \times 64$, flip angle $=90^{\circ}$ ). Voxel size was $3.5 \times 3.5 \times 4$ $\mathrm{mm}$. A gradient echo image $\left(\mathrm{TR}=704 \mathrm{~ms}\right.$, TE 5.11 and $7.57 \mathrm{~ms}$; flip angle $=60^{\circ}$ ) with the same grid and slice orientation as the functional images was acquired to generate a field map for correcting susceptibility-related distortions in the functional images. A T1weighted anatomical scan was also acquired $(\mathrm{TR}=2250 \mathrm{~ms}, \mathrm{TE}=2.6 \mathrm{~ms}$, flip angle $=$ $90^{\circ}, \mathrm{FOV}=256 \mathrm{~mm}$, slice thickness $=1 \mathrm{~mm}$, matrix size $=256 \times 256$, number of slices =192). Voxel size was 1 x 1 x $1 \mathrm{~mm}$. Each participant was scanned in a task free run that lasted 10 minutes. The participant was instructed to fixate on a cross at the center of the screen, relax, and avoid movement.
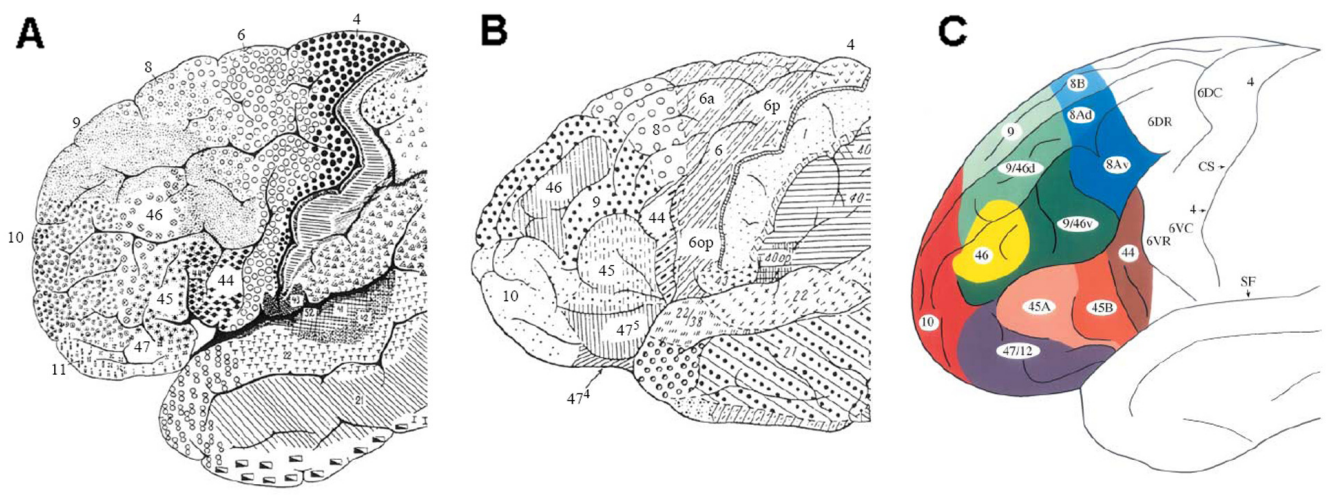

Figure 1. Cytoarchitectonic parcellations of the human lateral frontal cortex based on: A. Brodmann, 1909 B. Sarkissov et al., 1955 and C. Petrides and Pandya, 1994. Note the variability in the shape and extent of the same region in each parcellation scheme (e.g. region 46 in A and B). Note also the differences in the number of divisions (e.g. region 9 appears homogeneous in B, whereas it is further subdivided in C).

Preprocessing The fMRI data were preprocessed using the SPM software (Welcome Trust Center for Neuroimaging, London). Data were re-aligned, spatially corrected using the field map, slice time corrected, and co-registered with the anatomical scan. The individual T1-weighted anatomical scan from each subject was used for the coregistration with the functional volumes collected from the same subject. The T1weighted anatomical scans were segmented into 3 tissue types: grey matter, white matter and cerebrospinal fluid. The functional volumes were subsequently re-sampled to $3 \mathrm{~mm}$ isotropic voxels and smoothed with a $6 \mathrm{~mm}$ FWHM kernel. Normalization was not applied and the parcellation step (see below) was performed at the native space for each subject. The resting state data were additionally subject to the following preprocessing steps: 1) Removal of nuisance variables through multiple regression. Theses variables included the six motion parameters estimated at the re-alignment step, signal from the ventricles and signal from the white matter. 2) The residuals from this multiple regression were subsequently band passed $(0.01-0.1 \mathrm{~Hz})$. These preprocessing steps (1 and 2) aim at minimizing physiological effects such as respiration and heart rate and removing signal that is unlikely to have neural origin (Cordes et al., 2000; Van Dijk et al., 2010; De Luca et al., 2006). It should be noted that regression of the whole brain signal was not performed. 
Delineating the LFC patch The bias corrected anatomical scan from each subject was imported in Caret software. Each hemisphere was segmented and inflated and flat surfaces were generated. Each cortical patch was identified for each participant based on their individual anatomy. For the delineation of the patch the following boundaries were taken into account: Posterior boundary: the middle line of precentral sulcus (prCS). We chose a slight extension of the mask posterior to the prCS in order to ensure the full inclusion of the prefrontal regions that are positioned anterior to the premotor cortex. Superior boundary: the fundus of the superior frontal sulcus (SFS). Inferior boundary: To ensure the inclusion of Broca's area (BA 44 and BA45) the inferior end of the region of interest was defined as halfway between the lateral aspect of the Sylvian fissure and the circular sulcus. This compromise was chosen to accommodate substantial intersubject variability in the location of the inferior part of BA 44 and 45 in the Sylvian fissure, which ranges from just inside the fissure to its fundus at circular sulcus (Amunts et al., 1999, Uylings et al., 2005). The orbital part of the inferior frontal gyrus (IFG) was excluded by using the horizontal ramus as a macroscopic landmark (Uylings et al., 2010). Anterior boundary: The anterior boundary roughly followed pragmatic cut-offs previously proposed that aim at the identification of the posterior border of the frontopolar cortex (parts of region 10), namely a vertical plane in between the anterior part of the cingulate sulcus and the anterior part of the olfactory sulcus (Uylings et al., 2010). With the specified borders, the LFC patch excluded regions that extend medially (superior frontal gyrus (SFG) / area 9 and 8), the most anterior part of the frontal lobes and the orbital part of the IFG. The extent of the cortical patch included frontal regions that are reported as major nodes of networks responsible for the execution of diverse tasks (Stiers et al., 2010; Duncan and Owen, 2000).

Functional connectivity based parcellation It has been demonstrated that rsfMRI can be used in order to delineate subregions within a large anatomical structure. Despite that FC based on rsfMRI does not necessarily reflect structural connectivity it is constrained by the later (Honey et al., 2007, 2009). Moreover, multimodal parcellation based on both structural and functional connectivity leads to converging results (Zhang et al., 2010). The usefulness of rsfMRI data for the parcellation of a cortical region is reflected for instance in Margulies et al., 2009 where the time course of regions of interest (ROIs) placed in the precuneus of the human and macaque brain, and the subsequent application of spectral clustering, was able to reveal subdivisions of the precuneus by grouping together ROIs that had similar time courses. A similar approach can be applied to the voxel level, namely creating subregions by grouping voxels together based on the similarity of their rsfMRI time course. Such an approach has been demonstrated to lead to meaningful divisions of the visual cortex and intraparietal sulcus (Shen et al., 2010). In this study we adopt a voxel wise high resolution approach. Such an approach does not require the a priori specification and placement of a set of ROIs, and thus allows the investigation of the structure of a cortical patch in an unbiased and data-driven fashion. Moreover, the parcellation was conducted separately for each subject and hemisphere. The subject-wise approach avoids potential biases from across subject averaging usually applied prior to the parcellation procedure. The cortical patch (one for each hemisphere) from each subject was used as a mask in order to extract the rsfMRI time course of every voxel included in the mask. A $\mathrm{NxN}$ correlation matrix, where $\mathrm{N}$ is the number of voxels included in the mask, was computed by correlating the rsfMRI time course of each voxel with the time course of every other voxel in the mask. The number of voxels $\mathrm{N}$ varied between 2000 to 2500 approximately from participant to participant. As a measure of correlation Pearson's correlation coefficient was used. The correlation 
matrix was subsequently used in order to trace distinct groups of voxels. It should be noted that an alternative way to formulate this correlation matrix, is to compute the correlations between the connectivity profiles of each voxel with the rest of the brain (e.g. Kim et al., 2010). In this way the correlation matrix reflects a "second order" similarity, and not the direct quantification of the similarity of the time course of each pair of voxels in the mask. This approach in formulating the correlation matrix led to similar results as the ones reported below.

There are several ways to trace distinct groups from the correlation matrix. Approaches previously used include the k-means clustering algorithm (Kim et al., 2010; Beckman et al., 2009), spectral clustering (Kelly et al., 2010), and spectral reordering (Klein et al., 2007). Here we chose to employ an algorithm that belongs to the so called module detection algorithms. Module detection algorithms stem from graph theory. A correlation matrix can be considered as a graph where the $\mathrm{N}$ voxels can be considered as the nodes of the graph, and the correlation coefficient between the rsfMRI time courses of the ith and the jth voxel can be considered as an undirected edge between the ith and jth node. Module detection algorithms seek to maximize the modularity value $Q$ (Newman, 2006):

$$
Q=\sum_{i=1}^{k}\left[\frac{e_{i}}{m}-\left(\frac{d_{i}}{2 m}\right)^{2}\right]
$$

with $e_{i}$ standing for the number of edges within module $i, d_{i}$ for the total degree, i.e. number of functional connections/edges, of the nodes belonging to module $\mathrm{i}$, and $\mathrm{m}$ for the total number of edges in the graph. Hence, by seeking to maximize the value of Q, the algorithm seeks to trace communities/modules that exhibit more links/edges, i.e. in the current context functional connections, than the ones expected by chance. The modularity maximization is an NP-complete problem and various algorithms have been proposed in order to approximate the maximum Q value (Fortunato, 2010). Module detection algorithms have been applied in various networks including biological and social (Fortunato, 2010). Recently such algorithms have also been applied to functional networks derived from neuroimaging data (Meunier et al., 2009; Rubinov and Sporns, 2010; Barnes et al., 2010). Here we employ the so called Louvain module detection algorithm (Blondel et al., 2008) for the following reasons: 1) It is a data driven algorithm, like all the module detection algorithms, and hence it does not require the a priori specification of groups to be identified (as it is required e.g. with the k-means algorithm). This is particularly useful when no clear evidence exist with respect to the distinct groups that must underlie the data, like it is the case in the current study. 2) This algorithm was identified among the best performing ones in a comparative study that included a large variety of different community/module detection algorithms (Lancichinetti and Fortunato, 2009). 3) The algorithm is designed for fast and efficient module detection in large networks (Blondel et al., 2008). We used a Matlab implementation of the algorithm that is part of the Brain Connectivity Toolbox (Rubinov and Sporns, 2010).

Prior to the application of the algorithm, the correlation matrix needs to be thresholded in order to retain the stronger edges/correlation coefficients and form the graph to be used in the subsequent analysis (e.g. Barnes et al., 2010). No "gold standard" exists with respect to the threshold applied and a common tactique is the application of a range of thresholds. Here we use 3 "threshold levels" corresponding to 
0.5, 0.6 and 0.7 threshold values. At each level only the correlation coefficients above the corresponding threshold are retained. In all of these threshold levels the graph connectedness was equal to 1, i.e. no fragmentation of the graph occurred. For each threshold level the Louvain algorithm was applied. Due to the stochastic nature of the algorithm several runs $(=50)$ were applied. Thus, for each threshold level 50 parcellations were obtained with a corresponding Q value. In order to select the "best" threshold level we focused on the level that yielded the highest $Q$ values and hence leads to a more modular description of the data. Subsequently the "best" partition out of the 50 partitions corresponding to the "best" threshold level was selected as the one with the maximal Q value (e.g. Sporns et al., 2007). This partition was used for the results reported below. Since the analysis was performed at the voxel level, each voxel in the specified cortical patch was assigned a unique label indicating the module that has been assigned to. Hence by mapping the obtained partition to 3D space we obtain the results in the form of a "module map" for each participant and hemisphere separately. By overlaying this "module map" on the individual anatomy of each subject we can have a detailed view of the distinct subregions of the LFC patch (see Results). We will use the terms module(s) and subregion(s) interchangeably.

Control analyses In order to check the significance and robustness of the obtained parcellations a series of control analyses took place. For each threshold level the module detection algorithm was also applied to 10 matched random graphs, i.e. matched to the original graph in number of nodes, edges and degree distribution. The resulting $\mathrm{Q}$ value, i.e. the average value obtained in the 10 random graphs, offers a "baseline" value that expresses the expected null Q value. A similar control analysis has been employed in recent neuroimaging studies that employ module detection algorithms (e.g. Meunier et al., 2009).

Another approach that can be followed in order to investigate the quality of the partition that was recovered is to examine the robustness of this partition to perturbations of the graph (Karrer et al., 2008). The underlying rationale of this approach is that perturbations of the graph, which are accomplished through rewiring of the edges with a certain rewiring probability, should not have a detrimental effect on the structure of the graph. Hence the parcellations obtained from the original and perturbed graph should not vary considerably. As a reference point in this approach matched random graphs that function as null models are used. The variation of the parcellations obtained before and after the perturbation is quantified as more severe perturbations occur, i.e. edges are rewired with higher probability. The variation of information is used in order to compare the similarity of the parcellations (Meila, 2007). If the parcellations of the increasingly perturbed original graph exhibit less variation compared to the ones that correspond to the increasingly perturbed random graphs, we can conclude that the identified partition in the original graph is a robust structure.

The above control analyses concern the obtained partition as a whole. In order to examine the significance of each detected module at the individual level we employed the method proposed in Lancichinetti et al., 2010. Given a graph and a detected module, this method estimates the likelihood of finding such a module in an equivalent random graph. The so called B-score expresses this likelihood, with a value of less than $5 \%$ indicating a significant community in real world applications (Lancichinetti et al., 2010). An interesting aspect of this approach is that given a level of significance q, we can derive the largest subset of a module satisfying this level of significance by "peeling off" the worst nodes of the module. In our case, the modules detected by the Louvain algorithm were subject to this module-wise test of significance at 0.05 . Modules that 


\section{Chapter 2}

failed to meet this score were "peeled off" until they complied with the specified level of significance $(<0.05)$. All modules reported below satisfy this significance level.

The modularity optimization is characterized by degeneracy, i.e. many topologically distinct partitions can correspond to high modularity values (Good et al., 2010; Rubinov and Sporns, 2011). The detailed exploration and enumeration of these "degenerate partitions" is beyond the scope of the paper. Instead, a basic control analysis was conducted in order to examine the degree of similarity of the "best" partition and a distribution of "control partitions" (Liang et al., 2011). For each participant we computed $2^{\mathrm{m}}$ control partitions, which is near the order of the low bound of the degeneracy of Q (Liang et al., 2011; Good et al., 2010), where $\mathrm{m}$ is the number of modules of the "best" partition. Subsequently the similarity of the "best" with the "control" partitions was quantified with the variation of information. We also examined the distribution of the number of modules and the $\mathrm{Q}$ values, along with the variation of each module in the "best" partition, across the "control" partitions.

Some extra control analyses also took place in order to examine the effect of the threshold level applied to the correlation matrix, and the smoothing applied to the fMRI data. To this end, the variation of information was calculated between the parcellations reported below and 1) parcellations obtained at different threshold levels and 2) parcellations derived from unsmoothed rsfMRI data.

Group wise clustering In order to summarize the parcellation results across participants we employed a clustering approach that aimed to group similar modules across participants together. Since the parcellation was performed at the native space for each subject separately, prior to the group wise clustering, the module map of each subject was normalized to the MNI space. In order to preserve discrete labelling the nearest neighbour interpolation was used. As a measure of similarity between module $\mathrm{i}$ and $\mathrm{j}$ a "mixed distance" measure can be employed that combines spatial and connectivity similarity. As a measure of spatial similarity the Euclidean distance of the center of mass (COM) of module $i$ and $j$ was used. For connectivity similarity we first computed the whole-brain FC for each module. In order to avoid size biases, since the size of the identified modules was not exactly the same, we centred a spherical ROI (4 $\mathrm{mm}$ radius) at the voxel with the highest within module $\mathrm{z}$-score. The within module $\mathrm{z}$ score is defined as the number of connections $\mathrm{k}$, in our case functional connections, that a node $i$, in our case a voxel, has with its assigned module $\mathrm{m}$, minus the average of the within module connections $\mathrm{K}$ of all nodes of module $\mathrm{m}$, divided by the standard deviation of K (Guimera et al., 2005). Hence, higher values of the within module zscore for a voxel indicates that this voxel is more tightly connected with its assigned module and therefore constitutes a "good member" of the module. Consequently, the average rsfMRI time course of the spherical ROI centred at the voxel exhibiting the maximum within module z-score is a "representative rsfMRI time course" of each module. Pearson correlation coefficients between the "representative rsfMRI time course" of each module and the rsfMRI time courses of the N voxels in the brain were computed. Hence, for each module, a 1XN vector was obtained that describes the FC of each module with the rest of the brain. Such vectors will be refered to as module FC profiles. The connectivity similarity between module $i$ and $j$ was estimated as 1-r, where $r$ is the Pearson correlation coefficient between the Fischer $r$ to $z$ transformed values of the FC profile of module $i$ and $j$. Hence smaller values between module $i$ and $j$ indicate more similar FC with the rest of the brain.

By computing all pair wise spatial and connectivity similarities for all the modules $\mathrm{M}$ we obtain 2 MXM similarity matrices: D spatial and D connectivity. We can combine 
the 2 measures in 1 by forming a mixed similarity matrix $D$ that is a weighted sum of $D$ spatial and $\mathrm{D}$ connectivity. The relative contribution of each type of similarity is controlled by the parameter $\mathrm{w}$, with $\mathrm{w}=0$ leading to pure connectivity similarity and $\mathrm{w}=1$ leading to pure spatial similarity (eq. 2).

$$
D_{i j}=w \cdot \operatorname{Dspat}_{i j}+(1-w) \cdot \operatorname{Dconn}_{i j}
$$

Hence entry Dij captures the final similarity measure between module $\mathrm{i}$ and $\mathrm{j}$. Here we used as default the pure spatial similarity $(\mathrm{w}=1)$ and all the results are based on this type of similarity. The spatial similarity, i.e. Euclidean distance, between detected subregions/modules has also been recently used as a "cost" for grouping similar subregions across groups, albeit with a different algorithm than the one we employed in this study (Barnes et al., 2011). Explorations of a range of values of parameter $w$ showed no detrimental effects on the group clustering procedure (data not shown). It should be noted that in case of a mixed similarity measure the 2 measures must be normalized so that their relative contribution relies solely on parameter $\mathrm{w}$ and not on differences of the range of values.

The resulting similarity matrix was used in order to construct a dendrogram with an average linkage procedure (Unweighted Pair Group Method with Arithmetic Mean). Subsequently, group wise clusters of modules were formed in the following way: Modules with the smaller distance were put in the same cluster if they belonged to different subjects. No more than one module per subject was allowed to be a member of the same cluster. The dendrogram was progressively "climbed up" and in that way modules were visited in descending order of similarity. A cluster was finalized when the number of modules included reached a pre-specified number of subjects (here we used the total number of participants) or when there were no more modules to consider. The modules belonging to the finalized cluster were excluded and the procedure was repeated until no modules were left. The approach that is followed here for grouping similar modules across participants resembles other approaches employed to group Independent Component Analysis components (e.g. Esposito et al., 2005). In that way clusters of modules were formed in a data driven way. The COM of each cluster of modules was calculated as the mean $\mathrm{COM}$ of the modules belonging to the cluster.

\section{Examination and characterization of subregions}

Whole-brain FC In order to gain insight into the FC of each identified cluster of modules, and consequently its potential identity and role, exploratory data analysis took place by computing whole-brain FC maps for the identified clusters of modules (e.g. Kelly et al., 2010). The values of the FC profiles of each module belonging to the same cluster were transformed using Fischer's $\mathrm{r}$ to $\mathrm{z}$ formula and stored as a niftii image. Subsequently these images were smoothed with a $6 \mathrm{~mm}$ FWHM kernel and were subject to a one sample t-test against the null hypothesis. The resulting maps were thresholded at a False Discovery Rate (FDR) level $(\mathrm{q}<0.05)$ and represent the wholebrain FC of each cluster of modules. It should be noted that these maps are derived from "single" regression models, i.e. no regressors accounting for variability explained by other clusters of modules were used.

LFC families Additionally, an exploratory data analysis was performed in order to trace distinct LFC "families" (Passingham et al., 2002) through the quantification of the similarity of the whole-brain FC of the clusters of modules and thus unveil the intrinsic 


\section{Chapter 2}

LFC architecture at a higher level. A representative FC profile was computed for each cluster of modules by averaging the $\mathrm{r}$ to $\mathrm{z}$ transformed FC profiles of the modules constituting each cluster. As a distance measure we used 1-r and the clusters were arranged with the Kamada-Kawai spring embedding algorithm (e.g. Nelson et al., 2010) as implemented in the Pajek software. This results in the placement of clusters with (dis)similar whole-brain FC (apart) close to the Euclidean space. Thus, this arrangement offers a "raw" representation of whole-brain FC similarities. Moreover, for the formal identification of separate groups (families) with similar whole-brain FC, an average linkage was employed in order to construct a dendrogram that captures the similarity of the whole-brain FC of the clusters of modules. In order to assess how good the dendrogram captures the raw similarities of the data the cophenetic correlation coefficient was used (e.g. Palomero-Gallagher et al., 2009) with higher values indicating more faithful representations of the raw data. Families of clusters were identified if the intra-family distance was less than $70 \%$ (default value in Matlab) of the largest distance in the dendrogram. Moreover, in order to ensure the robustness of the traced families, we grouped the correlation matrix derived from all pair-wise correlations of all the cluster representative FC profiles with the k-means algorithm (e.g. Kim et al., 2010). Since the number of clusters $\mathrm{k}$ has to be defined in advance, we run the algorithm for $\mathrm{k}=2,3 \ldots 6$. Variations of the solutions obtained after the application of the k-means algorithm can be observed due to the random initiation of the cluster means. Hence, for each k, 1000 solutions were computed. Subsequently, the silhouette metric was used in order to assess the quality of the clustering (e.g. Kelly et al., 2010). The silhouette metric quantifies the quality of the clustering by assessing how dissimilar a data point is with respect to the cluster that is has been assigned to and all the other clusters, with values ranging for -1 (low quality) to 1 (high quality). Hence, the average silhouette across the 1000 solutions for each $\mathrm{k}$ was computed and the $\mathrm{k}$ that gave rise to the higher silhouette was selected. It should be noted that we only took into account the number of $\mathrm{k}$ clusters that resulted in solutions with no singletons, i.e. all clusters should contain at least 2 items. This is a reasonable constrain since a $\mathrm{k}$ equal to the number of data points will result in a trivial solution, with a silhouette of 1 , with every data point constituting a cluster on each own. Finally, in order to assess the stability of the families revealed by the k-means algorithm, a "frequency of co-clustering" matrix was constructed, where its entry $\mathrm{i}, \mathrm{j}$ denotes how many times across the 1000 solutions the cluster of modules $i$ was part of the same family with the cluster of modules j. Similar techniques were used for the grouping of regions in the medial wall of the macaque brain (Hutchison et al., 2012). The current approach, i.e. the parcellation into modules and the subsequent grouping into families, which offers a view of the LFC intrinsic architecture at multiple levels (e.g. Doucet et al., 2011), resemble techniques used for the parcellation of the human parietal cortex (Nelson et al., 2010).

To assess differences in the FC of different clusters, and thus illustrate potential distinct functional roles, separate t-tests were used. Since exhaustive pair-wise contrasts between all clusters are impractical we used evidence from the literature for the selection of clusters. More specifically, we focus on clusters on the ventral and dorsal LFC. We contrast clusters that seem to correspond to the so called Broca's region and seem to have different roles in the language domain (e.g. Amunts et al., 1999; Kelly et al., 2010). At the ventral part, we also examine clusters located within the triangular part of the inferior frontal gyrus (IFG) and the inferior frontal sulcus (IFS), to elucidate FC transitions from Broca's region to more dorsolateral subregions of the LFC (e.g. Amunts et al., 2010; Rajkowska and Goldman-Rakic, 1995; Kelly et al., 2010). Finally, 
we focus on clusters of modules located at the dorsal LFC at a location were there is evidence for the interfacing of distinct major large-scale networks (e.g. Corbetta et al., 2008; Buckner et al., 2008) for which the borders of their respective LFC subregions are not well delineated and examined.

For a schematic overview of the analyses pipeline see Fig. 2. All the above analyses were performed with a combination of custom software written in Matlab (Mathworks) and freely available software.

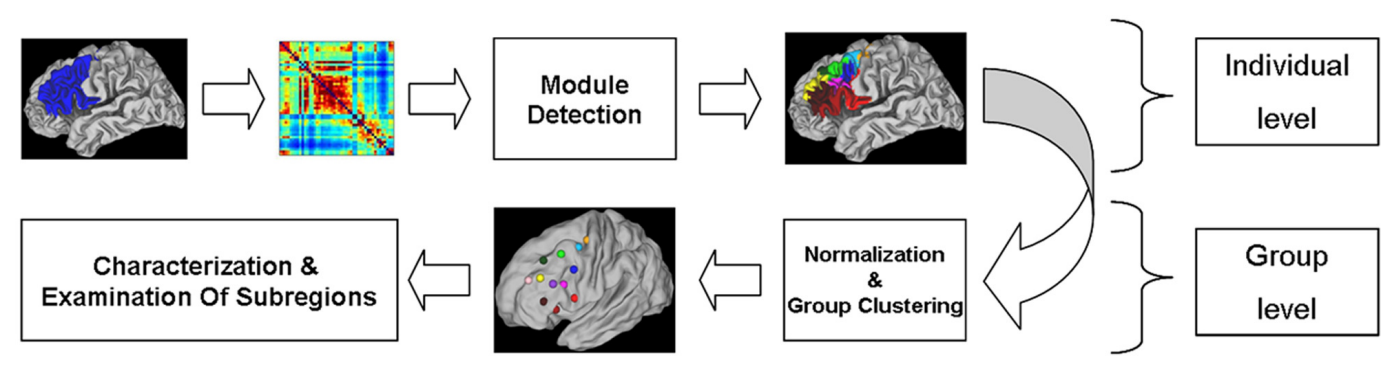

Figure 2. Schematic representation of the analysis pipeline followed in the current study (see Materials and Methods for details).

\section{$\underline{\text { Results }}$}

Functional connectivity based parcellation and control analyses Results reported below concern the left hemisphere due to practical reasons and the existence of cytoarchitectonic probabilistic maps for regions 9 and 46 only for this hemisphere (Rajkowska and Goldman-Rakic, 1995). This also makes possible the comparison of the current results in the light of previous parcellations (Kelly et al., 2010). Quantification and discussion of potential differences between the hemispheres, e.g. the left hemisphere seems to be more specialized (Iturria-Medina et al., 2011), are out of the scope of the current study. Hence, despite that comparable results were obtained for the right hemisphere, the intrinsic functional architecture of the LFC should not be considered identical for the two hemispheres.

The parcellation resulted in contiguous distinct subregions/modules within the LFC patch for all the participants. The resulting module maps are depicted in Fig. 3 overlaid on the individual anatomy of 4 participants. Despite the differences in size and extent, the module maps had a similar layout across participants.

Higher Q values were obtained at the highest threshold level (Fig. 4 A) and the results reported reflect the parcellation obtained at this threshold level. The number of modules detected varied from participant to participant (mean: $9.83 \mathrm{sd}: 0.93$ ). This variation can stem from the fact that pragmatic borders were used for the delineation of the LFC patch based on macroscopic landmarks. Hence, bits of the frontopolar and the premotor cortex, the most anterior and posterior boundaries of the patch, could be included in some participants and deemed as a separate subregion (see also Discussion).

The obtained $Q$ values were high $(>>0.3)$ which is indicative of the presence of a modular structure (Fortunato, 2010) (mean: $0.680 \mathrm{sd}$ : 0.074). Moreover, the $\mathrm{Q}$ values were much higher than the ones obtained from null models. These results are consistent with studies employing similar methods (e.g. Barnes et al., 2010; Meunier et al., 2009). The above held true for all threshold levels (see Fig. 4 A for a group summary). 
The perturbation analysis revealed that the obtained partition is a robust structure. As it is depicted in Fig. $4 \mathrm{~B}$, the similarity of partitions, quantified with the variation of information, obtained for the original graph at increasing levels of rewiring probability (more severe perturbations) in relation to the partition obtained from the unperturbed original graph, is much higher when compared to the ones obtained for a matched random graph.

Moreover, the detected modules are significant on an individual basis, as suggested from the B-scores $(<0.05)$ of each module. Only very few of the detected modules $(\sim 10 \%)$ were subject to a "peeling off" procedure in order to comply with the prespecified level of significance. Even in these cases only a handful of voxels were "peeled off" from each module.

The "control" partitions resulted in high Q values (mean: $0.662 \mathrm{sd}$ : 0.097). Despite the fact that these partitions were not identical with the "best" one, the variation of information was relatively low (mean: $0.065 \mathrm{sd}$ : 0.049). Furthermore, the number of modules of the "best" partition was the most frequent one, as assessed by the frequency distribution of the number of modules of the "control" partitions. Additionally, a very high percentage of the voxels constituting each module was always part of the same module across the "control" partitions (mean: $84.74 \%$ sd: 0.11 ). All values reported are across the participants. Finally, the parcellation results appear robust in choices of threshold level and the amount of smoothing of the fMRI data (data not shown).

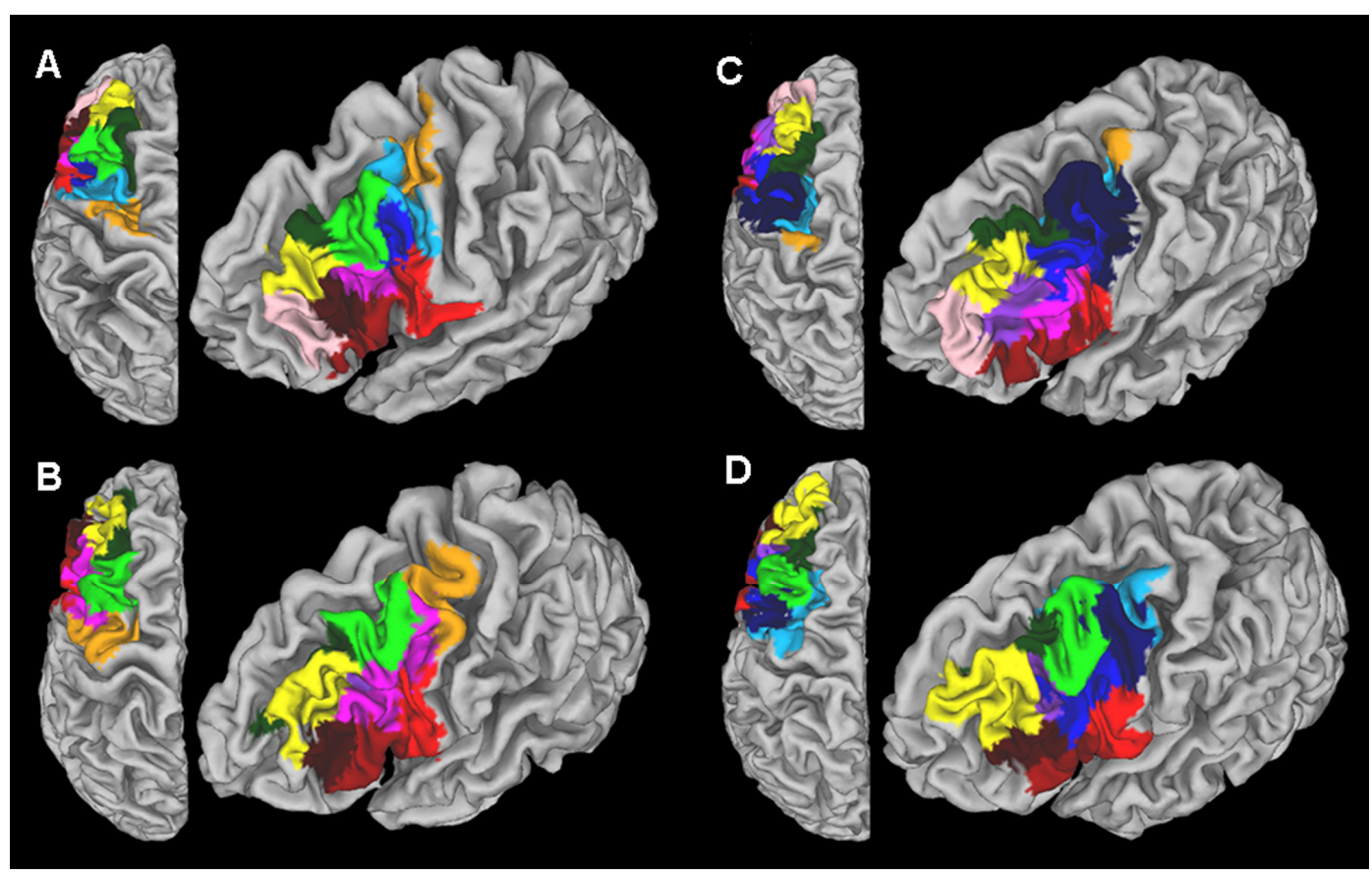

Figure 3. A-D. Parcellation results obtained for 4 subjects. Each module map is overlaid on the individual anatomy of each subject displayed in a lateral and dorsal view. Each module is coloured with a unique colour. Modules that are part of the same cluster of modules are coloured with the same colour (see Fig. 5). This colour coding will be followed throughout the paper. Note the similar layout of the modules across the subjects and the variability in the exact shape and extent of modules that belong to the same cluster of modules. Modules that did not end up in a "group representative" cluster of modules are coloured in dark (navy) blue (see Materials and Methods and Results). 
Taken together the above control analyses dictate that the parcellation results reported are robust and not heavily dependent on various methodological decisions and parameter selection.

Group wise clustering, examination and characterization of subregions The data driven clustering of the modules identified at the individual level was able to group similar modules across subjects. In order to summarize the results we will focus on this group wise clustering. In total 12 "group representative" clusters of modules were formed with each cluster containing at least modules from 7 out of 12 subjects (Table 1). Each cluster is assigned with a unique number from 1 until 12 (Cluster 1 (C1), Cluster 2 $(\mathrm{C} 2) \ldots$, Cluster $12(\mathrm{C} 12)$ ) in an arbitrary way (see Fig. 5). First we will describe the detected families of clusters of modules. This will offer a "family-wise" grouping wherein each individual cluster will be discussed. We will subsequently present the cluster comparisons that highlight FC differences and finally document each cluster in order to assign to each one a potential anatomical substrate.
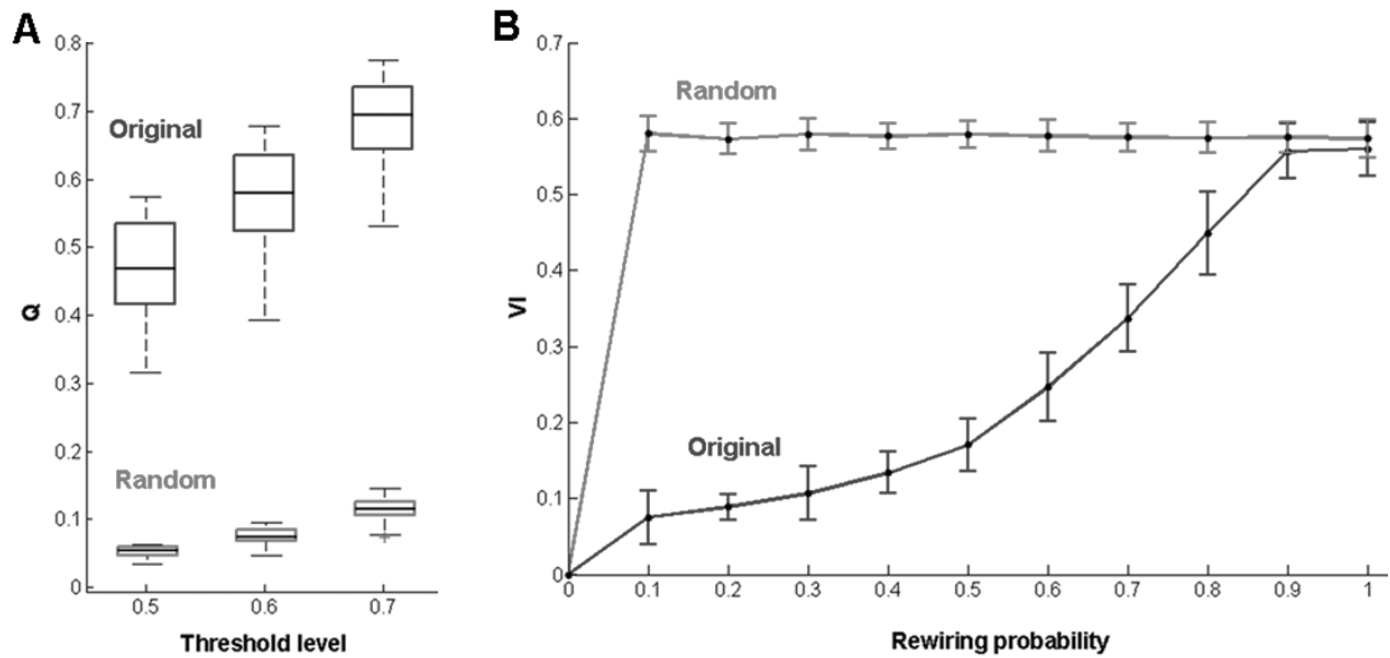

Figure 4. A. Modularity Q obtained at different threshold values for the original and equivalent random graphs. The box plots depict the median along with the $25^{\text {th }}$ and $75^{\text {th }}$ percentiles of the values. Whiskers represent minimum and maximum values and crosses stand for values marked as outliers. B. Variation of information between the parcellations obtained from the unperturbed and the increasingly perturbed graph, for the original and equivalent random graphs. Error bars represent standard deviations. Depicted values are across subjects (see Materials and Methods and Results).

LFC families The quantification of the similarity of the whole-brain FC of the clusters of modules resulted in 4 families, organized across the dorsal-ventral and anteriorposterior axis (Fig. 6). The degree of similarity within and between the families is also evident in the "raw" pairwise whole-brain FC similarities of the clusters of modules constituting each family (Fig. 6 A). Both clustering techniques (hierarchical and kmeans) identified the same 4 families (Fig. 6 B C). The dendrogram obtained from the hierarchical clustering (Fig. $6 \mathrm{~B}$ ) corresponds to a cophenetic correlation coefficient of 0.685 and hence it does not severely distort the original distances of the raw data. With respect to the k-means clustering the highest silhouette (0.693) for non singleton solutions was observed for $\mathrm{k}=4$ corresponding the exact same families traced by the 
hierarchical clustering (Fig. 6 C). Solutions for $\mathrm{k}=2$ and 3 gave rise to silhouette values of 0.540 and 0.563 respectively. Solutions for $\mathrm{k}>4$ contained singletons and thus were not taken into account. Moreover, the traced families always constituted a family across 1000 solutions as indicated by the frequency of co-clustering matrix which is dominated exclusively by entries with a value of 0 (never co-clustered) or 100 (always coclustered) (Fig. $6 \mathrm{C}$ ) illustrative of the robustness of the findings. Taken together the above results highlight the presence of 4 distinct families within the LFC, organized across the dorsal-ventral and anterior-posterior axis, that can be differentiated through their whole-brain FC. This differentiation is also evident in the FC maps of the clusters of modules constituting each family (Fig. 7).

Table 1. Summary of the clusters of modules for the left hemisphere.

\begin{tabular}{|c|c|c|c|c|c|c|c|c|c|}
\hline \multirow{3}{*}{$\begin{array}{l}\text { Cluster } \\
\mathrm{Nr}\end{array}$} & \multicolumn{6}{|c|}{ Center of mass } & & & \multirow{3}{*}{ Candidate anatomical substrate } \\
\hline & \multicolumn{2}{|l|}{$\mathrm{x}$} & \multicolumn{2}{|c|}{ 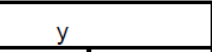 } & \multicolumn{2}{|l|}{$z$} & \multicolumn{2}{|c|}{ Volume $\left(\mathrm{mm}^{3}\right)$} & \\
\hline & mean & std & mean & std & mean & std & mean & std & \\
\hline 1 & $-54,90$ & 2,34 & 8,85 & 3,02 & 13,74 & 3,54 & $6.459,75$ & $2.289,03$ & BA 44 (Amunts et al., 1999) \\
\hline 2 & $-52,67$ & 1,23 & 25,09 & 3,74 & 3,32 & 2,52 & $4.949,10$ & $1.819,10$ & BA 45 (Amunts et al., 1999) \\
\hline 3 & $-46,42$ & 2,31 & 38,86 & 3,09 & 11,13 & 7,29 & $4.819,50$ & $2.449,03$ & $\begin{array}{c}46-45,9-45 \\
\text { (Rajkowska \& Goldman-Rakic, } \\
1995) \\
\text { 9/46v (Petrides \& Pandya, } \\
1994 \text { ) } \\
\end{array}$ \\
\hline 4 & $-32,28$ & 1,63 & 47,82 & 2,12 & 28,6 & 3,95 & $7.587,00$ & $2.668,66$ & BA 10 (Uylings et al., 2010) \\
\hline 5 & $-45,78$ & 4,57 & 19,40 & 5,62 & 23,29 & 4,89 & $7.503,00$ & $2.790,01$ & $\begin{array}{c}/ 46 v \text { (Petrides \& Pandya, } \\
\text { 1994) }\end{array}$ \\
\hline 6 & $-38,36$ & 3,26 & 17,73 & 4,95 & 52,42 & 2,43 & $6.361,87$ & $2.192,30$ & 8Ad (Petrides \& Pandya, 1994) \\
\hline 7 & $-35,60$ & 6,83 & $-9,02$ & 3,33 & 62,24 & 5,26 & $6.783,75$ & $3.154,68$ & Premotor BA 6 (Geyer, 2004) \\
\hline 8 & $-38,11$ & 5,21 & 37,17 & 4,83 & 31,27 & 3,00 & $6.169,50$ & $2.476,02$ & $\begin{array}{c}\text { BA 46 } \\
\text { (Rajkowska \& Goldman-Rakic, } \\
1995 \text { ) } \\
\end{array}$ \\
\hline 9 & $-34,17$ & 4,27 & $-0,94$ & 2,31 & 55,17 & 1,49 & $7.652,57$ & $2.597,65$ & FEF (Koyoma et al., 2004) \\
\hline 10 & $-29,42$ & 1,58 & 30,43 & 2,62 & 45,44 & 3,43 & $5.893,71$ & $1.875,26$ & $\begin{array}{c}\text { Lateral 9, 9-46 } \\
\text { (Rajkowska \& Goldman-Rakic, } \\
1995) \\
\text { 9/46d (Petrides \& Pandya, } \\
1994 \text { ) }\end{array}$ \\
\hline 11 & $-44,29$ & 4,19 & 7,15 & 2,44 & 38,02 & 4,48 & $7.205,14$ & $1.407,40$ & 8Av (Petrides \& Pandya, 1994) \\
\hline 12 & $-45,31$ & 3,56 & 25,95 & 10,35 & 26,77 & 6,86 & $6.861,85$ & $3.159,63$ & 9/46v (Petrides \& Pandya, 1994 \\
\hline
\end{tabular}

Cluster number refers to the corresponding cluster of modules (Fig. 5). The reported mean and standard

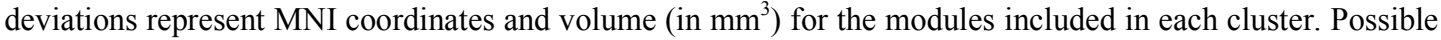
anatomical correlates of the modules of each cluster are provided along with relevant key reference(s).

A family located at the ventral part of the LFC was formed from the clusters of modules located across the IFS (C3, C5, C11, C12). These regions exhibit FC with regions that are part of the multiple demand/frontoparietal network recruited in various cognitive tasks (Fig. 7) (Duncan, 2010; Stiers et al., 2010; Vincent et al., 2008). The clusters located at the more dorsal parts of the LFC (C6, C10) formed a family with a "default mode" signature (Fig. 7) associated with internal processes (Buckner et al., 2008). The cluster of modules likely to correspond to region 46 (C8) (see Cluster documentation) formed a family with the more posterior clusters $(\mathrm{C} 1, \mathrm{C} 7, \mathrm{C} 9)$, with a premotor/occulomotor signature (Fig. 7), and not the immediately adjacent more 
anterior ones (e.g. Modules of C3, C10). This likely reflects the assumed role of region 46 in high levels of motor control (Goldman-Rakic, 1987; see Discussion). Finally, the more anterior clusters, i.e. C2 and C4, also formed a family exhibiting FC with the temporal cortex and the medial wall (Fig. 7) and are possibly implicated in audiovisual and semantic processing (see Discussion).

Interestingly, the families do not seem completely constrained by Euclidean distance, i.e. the clusters of modules of the same family can be remote from one another. For instance $\mathrm{C} 8$ is located more anterior than the rest of the clusters of the family that it belongs to (Fig. 6). Hence, subregions with similar whole-brain FC can be dispersed throughout the LFC (Yeo et al., 2011).

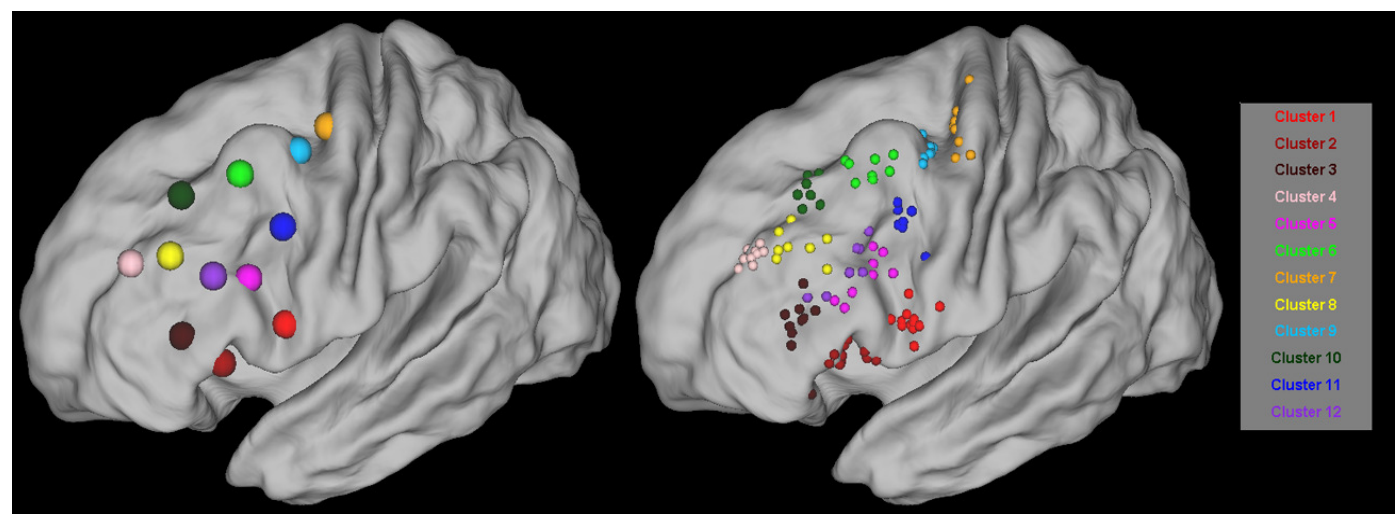

Figure 5. Large spheres represent the COM of the 12 clusters of modules. The COM of these clusters is the mean of the COM of the modules that constitute each cluster. Small spheres represent the COM of the modules of each cluster detected at each subject (see also Fig. 3). Only group representative clusters are depicted which contain modules from at least 7 out of 12 subjects. Each sphere is colour coded with a unique colour and the COM of modules belonging to the same cluster are coloured in the same colour.

\section{Cluster comparisons}

C1 vs $C 2$ Direct comparisons between the $\mathrm{FC}$ associated with $\mathrm{C} 1$ and $\mathrm{C} 2$, revealed significant differences. $\mathrm{C} 1$ exhibited more pronounced $\mathrm{FC}$ with the anterior part of the supramarginal gyrus (SMG), the intraparietal sulcus (IPS), the anterior part of the IFS, the medial part of the insula, the junction of the IFS with the superior frontal sulcus (SFS), and the supplementary motor area (SMA). C2 exhibited more pronounced FC with the anterior medial PFC, the angular gyrus (AG), the anterior temporal cortex and the posterior cingulate cortex (PCC) (Fig. 8 A, Fig. 7).

$C 2$ vs $C 3$ The direct contrast between $\mathrm{C} 3$ and $\mathrm{C} 2$ revealed higher $\mathrm{FC}$ of $\mathrm{C} 2$ with the triangular and orbital part of the IFG and the middle temporal cortex. Moreover, higher FC was observed with the superior frontal gyrus (SFG) extending towards the medial wall. C3 showed pronounced higher FC with the IFS with an extension towards the junction with the prCS, the junction of the SFS and the prCS, the anterior and posterior SMG, the IPS with an extension towards the occipital lobes, and the mid-cingulate cortex (MCC) (Fig. 8 B, Fig. 7).

C9 vs C6 and C10 Direct comparisons of the $\mathrm{FC}$ of $\mathrm{C} 9$ with $\mathrm{C} 6$ and $\mathrm{C} 10$ revealed pronounced differences. $\mathrm{C} 9$ compared to $\mathrm{C} 10$ was more functionally connected with the 
ventral prCS, the dorsolateral PFC, the IPS and parts of the occipital-parietal cortex (involving higher visual areas). Moreover, pronounced FC was observed with the anterior insula, the SMA and the cingulate motor regions (CMR) in the MCC, and parts of the postero-medial cortex likely to include Brodmann's region 7. The immediately adjacent $\mathrm{C} 10$, located anterior to $\mathrm{C} 9$, exhibited higher $\mathrm{FC}$ with the posterior part of the MFG, the AG, the SFG, the anterior medial PFC with parts of the ACC, the limbic region PCC and the orbitofrontal cortex (Fig. 8 C, Fig. 7). A very similar pattern of FC differences emerged when $\mathrm{C} 9$ was compared to adjacent C6 (data not shown).

These FC differences of adjacent clusters at the ventral and dorsal part of the LFC are consistent with observations of structural and FC in the human brain (Frey et al., 2008; Ford et al., 2010; Vincent et al., 2008) and connectivity of suggested homologues in the monkey brain (Petrides and Pandya, 2009; Pandya and Yeterian, 1996). They illustrate the FC differences, which are also observable in Fig. 7, that might relate to the different functional specializations associated with the subregions of each cluster (see Discussion).

\section{Cluster documentation}

In this section we will assign a potential anatomical substrate to each cluster. To this end, macroscopic landmarks, cytoarchitectonic maps, and the whole-brain FC of each cluster will be used in combination with known structural and functional connectivity of subregions of the human brain and candidate homologues in the macaque brain. The results reported concern the left hemisphere.

C1 The COM of the cluster is located anterior to the ventral part of the premotor cortex, in the depths of the prCS (Fig. 5). The modules of this cluster occupy the prCS and extent towards the opercular part of the IFG (Fig. $3 \mathrm{~B}$ red modules, 9 A, B). The coordinates of the COM are very near to the ones reported from a Diffusion Weighted Imaging (DWI)-based parcellation of BA 44 (Anwander et al., 2007) and a couple of millimetres caudally to the ROI used in a rsfMRI study investigating the FC of BA 44 (Kelly et al., 2010) (see Table 1). In addition, the COM falls within the region of the probabilistic map of BA 44 that exhibits high probability (Amunts et al., 1999) (see also Fig. 9 B). The FC map of $\mathrm{C} 1$ unveils extensive $\mathrm{FC}$ with regions located ventral to the IFS (Fig. 7). Extensive FC of $\mathrm{C} 1$ was observed with pre-SMA, SMA, and the anterior mid-cingulate cortex (MCC). Evidence from tracing studies suggest that region 44 in the macaque is connected with assumed homologues, more specifically with dorsal region MII, caudal 24 and the CMR (Paus, 2001; Pickard and Strick, 2001; Pandya and Yeterian, 1996). Moreover, a recent DWI study in humans also reveals structural connections of the posterior part of Broca's region (region 44) with SMA and preSMA in contrast the anterior part (region 45) which is anatomically linked with more anterior parts of the medial wall (Ford et al., 2010). Concerning the post-rolandic regions, prominent FC involved a big portion of the supramarginal gyrus (SMG), in line with connectivity of assumed homologues in the monkey brain (Petrides and Pandya, 2009, 2004). Lastly, FC was also observed with the intraparietal sulcus (IPS). This is consistent with evidence from the connectivity of the monkey brain (Petrides and Pandya, 2009). In addition, tracts connecting region 44 and the IPS have been traced in the human brain with diffusion weighted imaging (Frey et al., 2008).

C2 Modules belonging to this cluster occupy the triangular part of the IFG associated with BA 45 (Amunts et al., 1999) (Fig. 3 A dark red module, Fig. 9 A, B). The COM of this cluster (Fig. 5) is located within the probabilistic map of BA 45 (see also Fig. 9 B) 
and is close to the COM of a region identified as BA 45 in a DWI based parcellation (Anwander et al., 2007) and the coordinates of a ROI used to trace the FC of BA 45 (Kelly et al., 2010) (see Table 1). C2 exhibited extensive FC with a large part of the LFC (Fig. 7). With respect to regions outside the LFC, FC was pronounced with the lateral temporal lobe, the anterior part of the medial prefrontal wall and parts of the anterior cingulate cortex (ACC), and the posterior SMG extending towards the angular gyrus (AG). The FC of $\mathrm{C} 2$ resembles the connectivity of the assumed homologue of region 45 in the monkey brain.

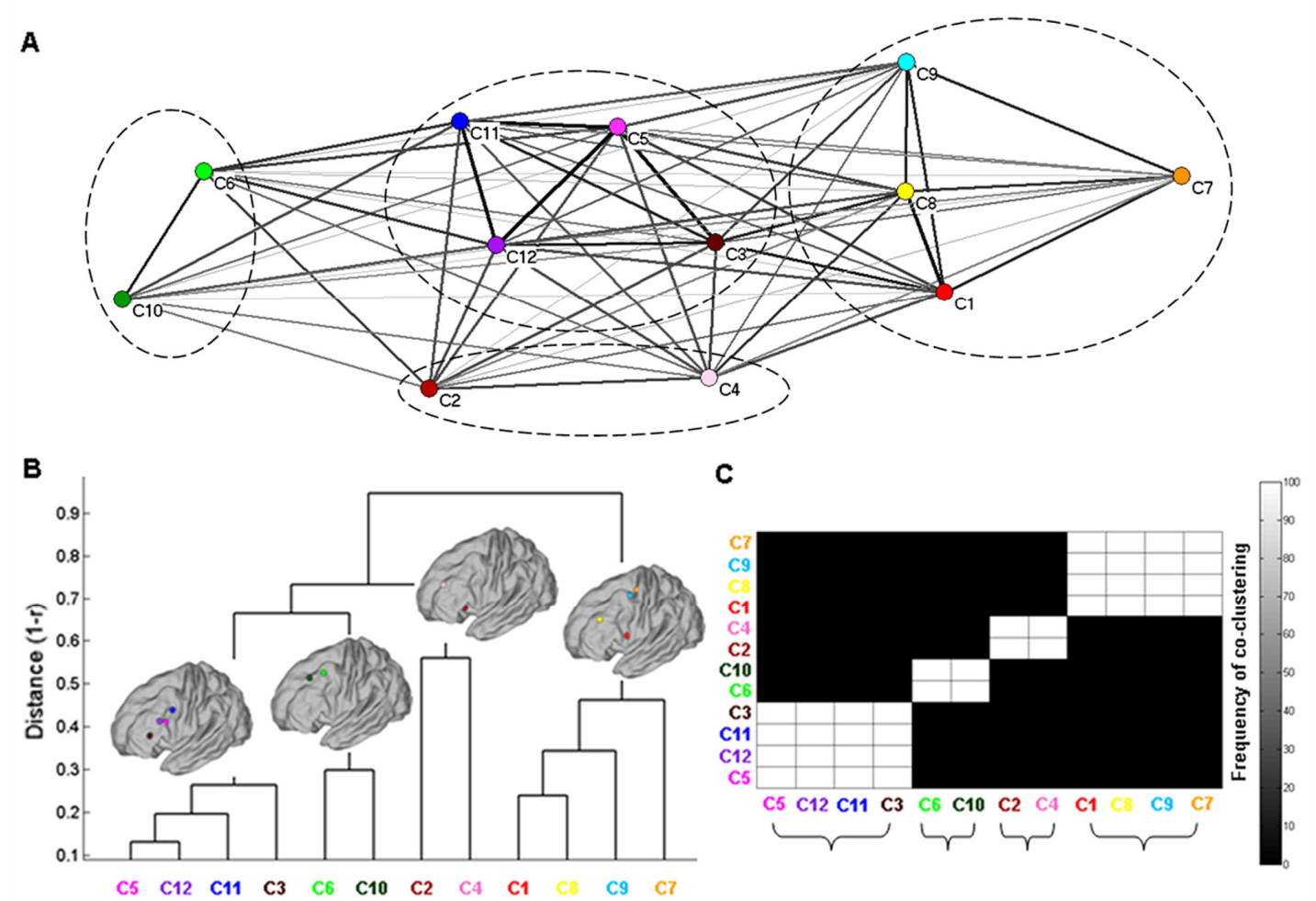

Figure 6. A. Representation of the "raw" similarity of the whole-brain FC between the traced clusters of modules. Clusters are arranged in space, by using the Kamada-Kawai spring embedding algorithm, based on the similarity of their whole-brain FC. Thick (thin) lines indicate high (low) similarity and circles delineate the families traced with hierarchical and k-means clustering. B. Dendrogram representing the similarity of the whole-brain FC of the cluster of modules. Each brain depicts the COM of the clusters of modules that form a "family", i.e. have similar whole-brain FC. A set of clusters of modules formed a family if the "intra-family" distance was less than the $70 \%$ of the largest distance in the dendrogram. C. Clusters of modules grouped according to their whole-brain FC similarity with the k-means algorithm. Brackets denote the grouping of the clusters of modules to families. The frequency of co-clustering matrix indicates the high stability of the families across $1000 \mathrm{k}$-means solutions (see Materials and Methods and Results for details).

The colour coding for each cluster of modules follows Fig. 5. More precisely, strong connections of macaque region 45 exist with various visual, auditory and multimodal areas located at the temporal cortex (Gerbella et al., 2010; Petrides and Pandya, 2009; Pandya and Yeterian, 1996). Tracers have also revealed connectivity of region 45 with area PFG in the macaque, which is the assumed homologue of the posterior part of the SMG in the human brain (Petrides and Pandya, 2009). Prominent connectivity of region 45 in the macaque has also been reported with the anterior part of the medial wall 
(Pandya and Yeterian, 1996). Interestingly, in line with the results of our study, a recent DWI study in humans revealed a posterior to anterior gradient with respect to connectivity of Broca's regions, i.e. regions 44 and 45, and the medial wall, with region 45 exhibiting structural connectivity with anterior parts of the medial wall (Ford et al., 2010).

C3 The COM of this cluster is located at the most anterior part of the IFS, dorsal to the triangular part of the IFG (Fig. 5). Modules belonging to this cluster are located at the tip of the IFS, extending dorsally towards the middle frontal gyrus (MFG) and ventrally towards the IFG (Fig 3 D brown modules, Fig. 9 A). Different cytoarchitectonic parcellation schemes have labelled differently the region adjacent to the dorsal part of the pars triangularis. In Brodmann's map this part of the cortex is labelled as region 46, whereas in Sarkissov et al., 1955 this part is labelled as region 9 (Fig. 1 A, B). Regions 46 and 9 exhibit considerable inter-individual variability which, along with the presence of many transitional zones with mixed cytoarchitectonic features, can contribute to the diversity of the various parcellation schemes (Rajkowska and Goldman-Rakic, 1995). In the later study, transition zones referred to as 46-45, 9-45 were observed at the fundus of the IFS dorsal to region 45 that resembles the position where C3 is located. Moreover, this patch of the cortex might correspond to the most anterior tip of region 9/46v of the Petrides and Pandya, 994 parcellation (Fig. 1 C). C3 exhibits FC mainly with the IPS, extending towards the parieto-occipital complex and the SMG. Extensive FC was also observed with the ventrolateral prefrontal cortex, IFS, the preSMA, and MCC (with parts of the CMR) (Fig. 7). Interestingly, a distinct cluster located dorsally to BA 45, extending across the IFS and exhibiting similar FC was traced in an rsfMRI-based parcellation (Kelly et al., 2010).

C4 The COM of the cluster is located most anterior to any other clusters identified (Fig. 5). The modules constituting this cluster are extending around the pragmatical posterior border for the frontal pole proposed in Uylings et al., 2010 (Fig 3 A light pink modules, Fig. 9 A). Consequently it seems the case that the identified modules of this cluster correspond to bits of the lateral frontopolar cortex, i.e. region 10 (Fig. 1 A). It exhibits FC with anterior MCC and posterior part of SMG (Fig. 7).

C5 The COM of this cluster is located at the IFS prior to the junction with the prCS, dorsal to the triangular and opercular part of the IFG (Fig. 5). The cluster consists of modules that primarily occupy the fundus of the IFS (e.g. Fig $3 \mathrm{C}$ fuchsia module, Fig. 9 A). The position of the modules corresponds to the cortical region labelled as region 9 (Sarkissov et al., 1955) (Fig. 1 B), and to transitional zones of region 9 (Rajkowska and Goldman-Rakic, 1995). Parcellation schemes that exploit the mixed cytoarchitectonic features of these transitional zones of region 9 have labelled this part of the cortex as region 9/46v (Petrides and Pandya, 1994) (Fig. 1 C). Extensive FC was observed with regions that are implicated in the execution of diverse tasks (Duncan, 2010) and are part of a network unveiled with rsfMRI termed as fronto-parietal control network (Vincent et al., 2008) (Fig. 7).

C6 The COM of this cluster is located at the posterior part of the MFG (Fig. 5). The modules that constitute this cluster occupy the most posterior part of the MFG (Fig. $3 \mathrm{C}$ light green module, Fig. 9 A). This part of the cortex is labelled as region 8 and it has as an anterior border region 9 (Brodmann, 1909; Sarkissov et al., 1955) (Fig. 1 A B). Cytoarchitectonic and variability analysis of region 9 indicates that the vertical plane located at $\mathrm{y}=+26$ (approximate coordinate in MNI space) can function as a conservative posterior limit of region 9 (Rajkowska and Goldman-Rakic, 1995). The identified modules of this cluster extent predominantly posterior to this plane. Moreover, the 
parcellation scheme of Petrides and Pandya 1994, labels the part of the cortex where the modules are located as region 8, and more specifically, as region 8Ad (Fig. 1 C). C6 exhibits extensive $\mathrm{FC}$ with the $\mathrm{AG}$, the anterior medial wall and $\mathrm{ACC}$, the orbital part of the IFG, the inferior temporal sulcus and the posterior part of the cingulate cortex (PCC) (Fig. 7). This FC map is very similar to the one characteristic of the so called default mode network (Buckner et al., 2008).

$C 7$ The COM of this cluster is located at the precentral gyrus and constitutes the most posterior cluster (Fig. 5). The modules constituting this cluster are primarily localized in parts of the cortex that exhibit high probability of belonging to region 6 (Geyer, 2004) (Fig. 3 A orange module, Fig. 9 A). The FC map of C7 includes large parts of the premotor and motor cortex, and is similar to maps derived from seed based approaches that involve motor related regions like the SMA (e.g. Kim et al., 2010) and posterior MCC (Fig. 7). Thus, it seems that modules that are part of this cluster correspond to bits of premotor regions in the most posterior part of the LFC mask.

C8 The COM of this cluster is located at the anterior part of the MFG (Fig. 5). The modules that constitute this cluster consistently occupy the convolutions of the anterior part of the MFG, with an occasional very moderate extension towards the SFS and the IFS (Fig. 3 A yellow module, Fig. 9 A, C). The position of the modules corresponds well with the location of region 46 delineated according to cytoarchitectonic properties and falls within conservative boundaries specified for this region (Rajkowska and Goldman-Rakic, 1995) (Fig. 9 C). Moreover, other cytoarchitectonic parcellation schemes also label this part of the prefrontal cortex as region 46, despite differences in the exact extent and shape of the region (Sarkissov et al., 1955; Petrides and Pandya, 1994; Brodmann, 1909) (Fig. 1). C8 exhibits extensive FC with various parietal (SMG, IPS), prefrontal (IFS), premotor and medial regions (MCC, SMA, preSMA, CMR, ventral premotor) (Fig. 7). This diffuse pattern of FC might give rise to the role that this region seems to play in diverge aspects of motor control and "higher order" processes (Goldman-Rakic, 1987; Lu et al., 1994; Petrides, 2005).

C9 This cluster consists of modules located near the junction of the SFS with the prCS (Fig. $3 \mathrm{C}$ cyan modules, Fig. 9 A). The junction of the prCS with the SFS has been identified as the locus of the frontal eye fields (FEF) in the human brain (Koyama et al., 2004). Its COM (Fig. 5) is very near to reported peaks of task induced activations involving a goal-directed visual search of a target stimulus (Asplund et al., 2010), attending, pointing and looking at a peripheral visual location (Astafiev et al., 2003) (Table 1). Moreover, the location of the modules belonging to this cluster is also consistent with the region that has been localized as the human FEF via electrical cortical stimulation (Blanke et al., 2000). It should be noted however that within this region there might be functional sub-specializations, as suggested by the presence of visuospatial maps (e.g. Hagler et al., 2007). C9 is located anterior to C7, which exhibits a more motor related FC, and posterior to clusters 6 and 10, which occupy portions of the posterior MFG and the SFS respectively. Hence, C9 is located in between portions of cortex most likely belonging to the anterior premotor cortex (BA 6) and portions of cortex that could correspond to regions region 9, 9-46 (Rajkowska and Goldman-Rakic, 1995), 8Ad, 9-46d (Petrides and Pandya, 1994) (Fig. 1 C). The FC map of C9 is highly similar to the FC map that is obtained by seeding from the FEF (Fox et al., 2006) and with the map corresponding to the dorsal attention system of which the FEF constitutes a core region (Vincent et al., 2008) (Fig. 7). This set of regions is similar to the network involved in smooth eye pursuit and saccades in the monkey brain (Tian and Lynch, 1996). 
C10 The COM of this cluster is located more anterior and dorsal to the one of C6 (Fig. 5). The modules of $\mathrm{C} 10$ extend across the SFS, occupying its fundus (Fig. 3 A dark green module, Fig. 9 A, D). The anatomical location of the modules matches the location of (lateral) region 9 and the transition zone 9-46, with the largest part of the modules falling within the conservative boundaries of region 9 (Rajkowska and Goldman-Rakic, 1995) (Fig. 9 D). Other cytoarchitectonic parcellation schemes label this part of the cortex as region (lateral) 9, 9/46d (Petrides and Pandya, 1994) (Fig. 1 C). The FC map of $\mathrm{C} 10$ was very similar to the FC map of C6, resembling the default mode network, and it encompasses parts of the PCC/retrosplenial cortex, dorsal and ventral medial PFC, ACC, AG, SFS and ventral temporal cortex (Fig. 7). This FC pattern resembles connectivity, as revealed by tracer studies, involving assumed homologues of region 9/46d in the monkey brain (e.g. Pandya and Yeterian, 1996).

C11 The COM of this cluster is located dorsal to the one of C1 (Fig. 5). The modules occupy the meeting point of the IFS and the prCS (Fig. 3 A blue module, Fig. 9 A). Different cytoarchitectonic parcellation schemes label this part of the cortex differently. In Brodmann's map this part of the prefrontal cortex is labeled as region 9 (Fig. 1 A). According to Sarkissov et al., 1955 the region adjacent to the dorsal part region 44 is labeled as region 8 (Fig. 1 B). Other parcellation schemes exploit cytoarchitectonic inhomogeneities of region 8 and introduce further subdivisions (Petrides and Pandya, 1994) (Fig. 1 C). According to the latter parcellation the region adjacent dorsal to area 44 is labeled as 8Av. The modules of C11 in our study do not resemble to region 9 since they do not extend towards the superior frontal gyrus and neither do they follow the pattern of region 8. Instead they have similar location and spatial extent as region 8Av according to the Petrides and Pandya, 1994 parcellation (Fig. 1 C). FC for C11 was observed with regions located ventrally (across the IFG) and dorsally (in the posterior part of the MFG extending to the posterior fundus of the SFS). FC was also observed with the IPS extending towards the AG, with the dorsal anterior MCC and with the temporal region just anterior to the MT+ complex (Fig. 7). It is interesting to note that the position of the modules of $\mathrm{C} 11$ is at the vicinity of the recent functionally defined region that is termed inferior frontal junction (Brass et al., 2005) and is recruited during diverse cognitive tasks (Stiers et al., 2010). This region has also been demonstrated to exhibit distinct recepto- and cyto-architectonic properties (Amunts et al., 2004a).

C12 The COM of this cluster is located anterior to the one of C5 (Fig. 5). The modules constituting this cluster were located dorsal to the triangular/opercular part of the IFG (Fig. 3 D violet module, Fig. 9 A). The possible anatomical correlates resemble the ones described for C5. Its FC is also very similar with the one of C5 (Fig. 7).

The fact that $\mathrm{C} 5$ and $\mathrm{C} 12$ seem to correspond to the same cytoarchitectonic substrate might reflect functional subdifferentiations within cytoarchitectonically homogenous regions. This is in-line with deviations from a 1:1 relation of cytoarchitectonic and receptor, closely linked with functional aspects, based parcellations (Zilles and Amunts, 2010). Interestingly, task-based paradigms (Stiers et al., 2010; Badre and D' Esposito, 2009) and receptor mapping (Amunts et al., 2010) support the presence of separate regions across the IFS (possibly corresponding to region 9/46v). The investigation of these issues and the differences between functional sub-divisions of regions $9 / 46 \mathrm{v}$ can constitute the topic of future studies. 


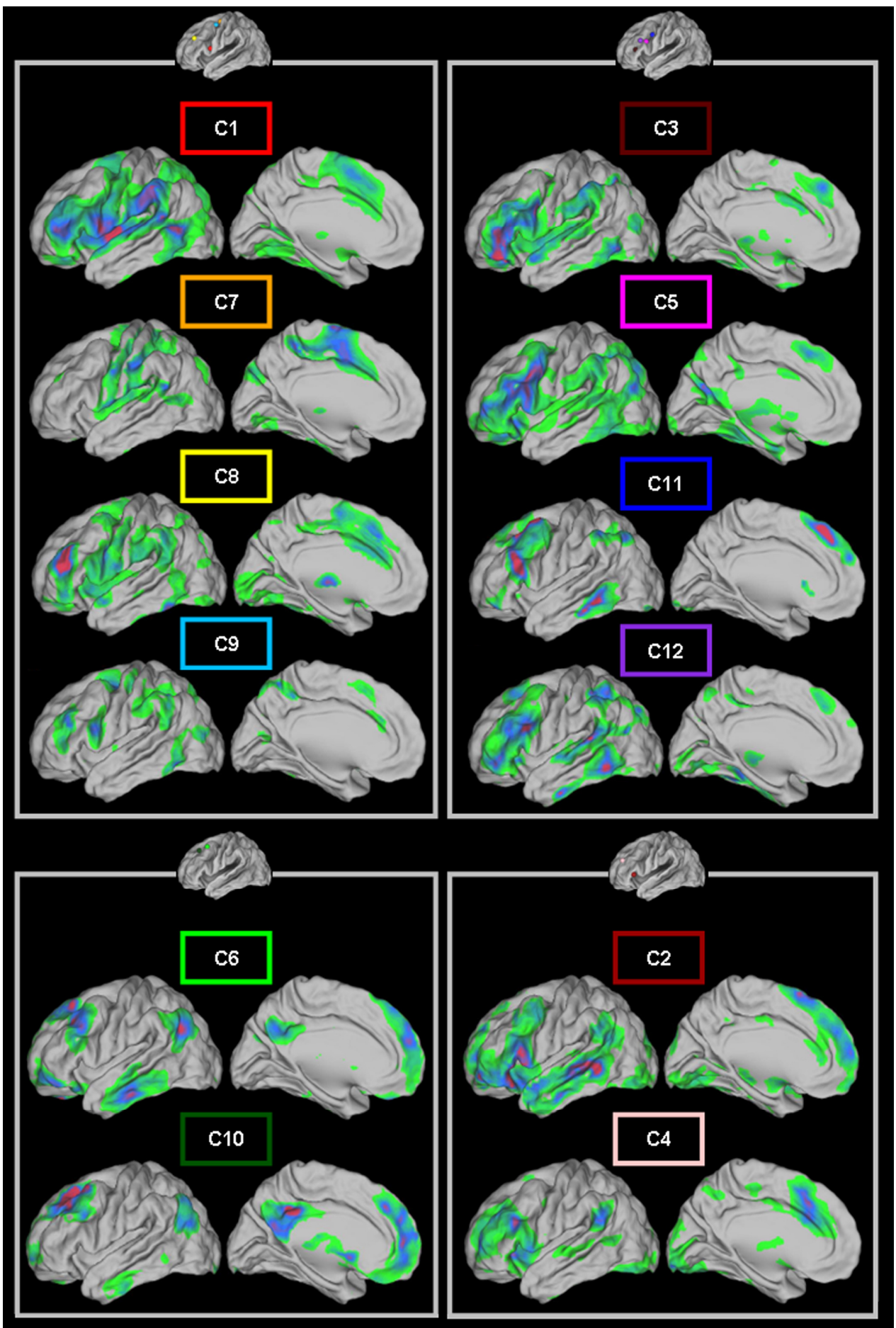

Figure 7. Whole-brain FC of the 12 cluster of modules grouped according to the 4 LFC families. Clusters are grouped according to the LFC family that they belong to (see Fig. 6) following the colour coding of Fig. 5. Darker (violet/red) regions represent higher $t$ values. All maps are thresholded at $\mathrm{q}<0.05$ (FDR level). Note the FC similarities (or dissimilarities) between clusters of modules that belong to the same (or different) family. For the computation of these "2nd level" maps different ROIs derived from the parcellation obtained from each subject were used (see Materials and Methods). 


\section{Discussion}

In the current study we delineated in vivo and on an individual basis subregions of the LFC with the aid of rsfMRI and data-driven algorithms. The subregions traced at the individual level were grouped with a data-driven across-subjects clustering, allowing the examination of the subregions at the "group level", which is a step forward from observer-dependent approaches (Barnes et al., 2010).

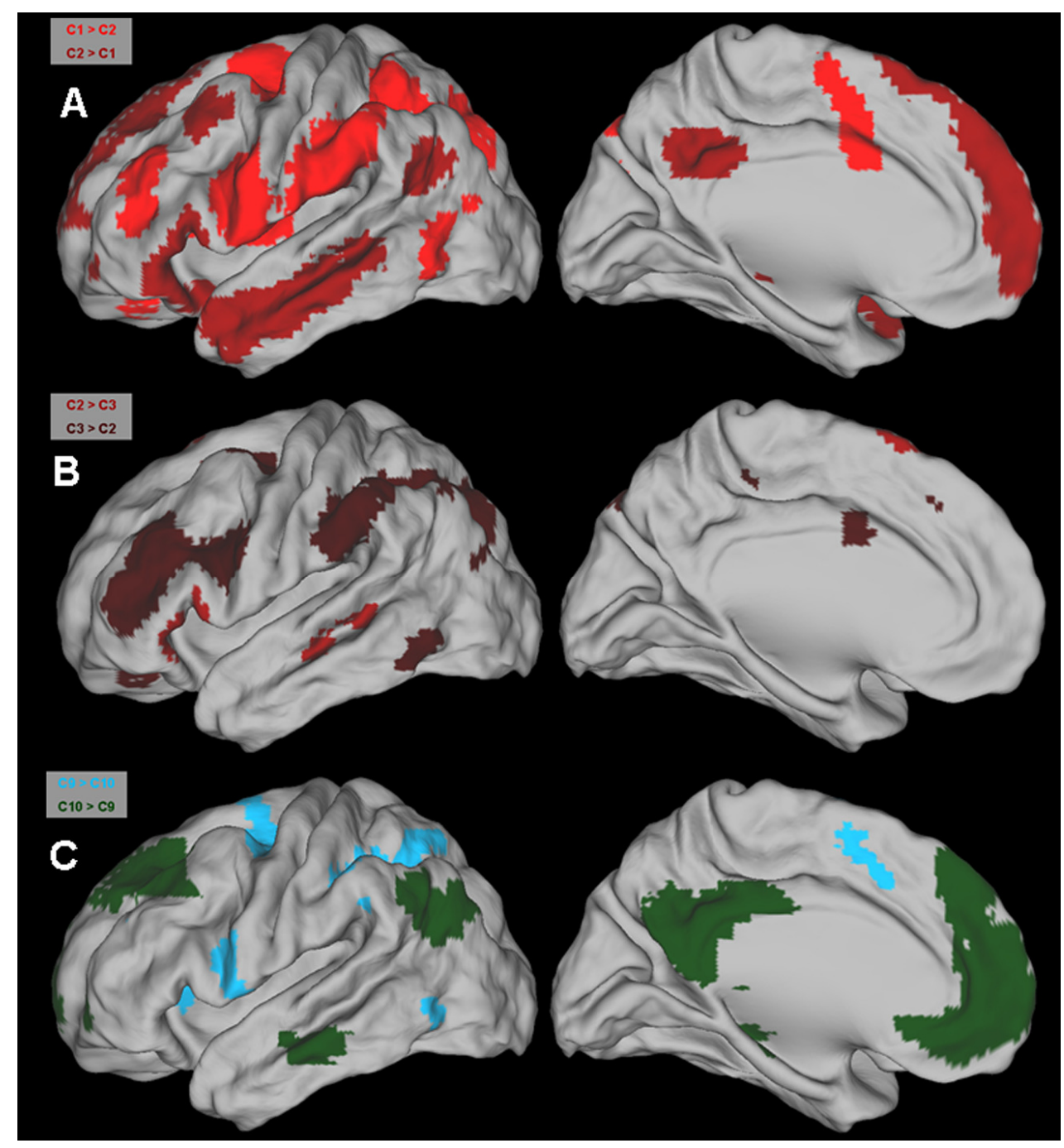

Figure 8. Direct comparisons through t-tests reveal significant differences in the whole-brain FC of clusters located at the A-B. ventral and C. dorsal part of the LFC. Significant differences associated with a particular cluster are coloured by using the colour coding of Fig. 5. Maps depict regions that are significant at $\mathrm{q}<0.05$ (FDR level).

Ventral and dorsal LFC clusters

Subregions of the ventral LFC, that seem to correspond to cytoarchitectonic divisions, namely regions $44(\mathrm{C} 1), 45(\mathrm{C} 2)$, i.e. Broca's regions, and transitional zones 46-45, 9- 
45 (C3) (Amunts et al., 1999; Rajkowska and Goldman-Rakic, 1995), exhibit distinct FC placing them into different families (Fig. 6, 7).

$\mathrm{C} 1$ and $\mathrm{C} 2$ show FC differences consistent with distinct connectivity patterns of assumed homologues in the monkey (Pandya and Yeterian, 1996) and structural connections in the human brain (Ford et al., 2010; Frey et al., 2008) (see Results and Fig. 8 A) that can account for separate functional roles in the language domain. Region 44 is involved in a neural circuitry responsible for hand and orofacial control and generation of speech acts (Ford et al., 2010; Petrides et al., 2005). Region 45 is connected with multimodal, auditory and visual areas (Gerbella et al., 2010; Pandya and Yeterian, 1996) and involved in semantic, audiovisual processing, and emotional embedding of auditory stimuli (Amunts et al., 2004b; Gerbella et al., 2010; Barbas, 2000).

C2 and C3 show significant FC differences (Fig. 8 B). Cytoarchitectonic analysis of region 45 (C2) indicates its occasional extension towards the ventral and dorsal banks of the IFS (Amunts et al., 1999). Cytoarchitectonic and receptor mapping studies pinpoint a separate region at the IFS, termed transition zone 46-45, 9-45 (Rajkowska and Goldman-Rakic, 1995) and ifs 1 (Amunts et al., 2010). The current parcellation is consistent with these studies, suggesting the presence of a subregion (C3) occupying the IFS, dorsal to the triangular IFG, with a distinct FC pattern from the one of region 45 (C2), involving a neural circuitry spanning visuospatial and sensory-occulomotor regions (Fig. 8 B).

Subregions of the dorsal LFC seem to correspond to FEF (C9), and regions 8Ad (C6) and lateral 9, 9/46d, 9-46 (C10) (Petrides and Pandya, 1994; Rajkowska and GoldmanRakic, 1995; Koyama et al., 2004), and belong to different families (Fig. 6).

Comparison of the FC of C9, located at the junction of the SFS and the prCS, to C10, located at the fundus of the SFS, reveal FC with regions of the dorsal attention system (Fox et al., 2006) (Fig. 8 C) involved in externally guided attention and occulomotor functions (Corbetta et al., 2008; Barbas, 2000). Comparison of the FC of C10 to C9 reveals FC with regions of the default network (Fig. $8 \mathrm{C}$ ) involved in "internal" processes like episodic memory retrieval (Raichle et al., 2001; Buckner et al., 2008). Similar FC differences emerged when contrasting the FC of C9 and C6 (not shown). The above illustrate the transition of whole-brain FC along the anterior-posterior axis at the dorsal LFC, involving distinct subregions belonging to different large-scale networks that obey a broad "externally/internally oriented" dichotomy (Vincent et al., 2008).

LFC families and FC with the cingulate cortex

Based on receptor profiles the cingulate cortex can be divided in 3 gross parts (Pallomero-Galagher et al., 2009) also characterized by functional specializations (Beckman et al., 2009): ACC, MCC and PCC/retrosplenial. Interestingly, the different LFC families exhibit preferential $\mathrm{FC}$ with different cingulate divisions.

C6 and C10 exhibit FC with the PCC/retrosplenial and ACC (Fig. 7). The PCC is associated with memory tasks (Beckman et al., 2009) and, compared to more anterior divisions, exhibits higher densities of acetylocholine which is implicated in memory formation (Pallomero-Galagher et al., 2009; Miranda et al., 2003). The ACC is associated with processes like mentalizing, linked with the default mode network (Amodio and Frith, 2006; Buckner et al., 2008). C3, C5, C11, C12, which correspond to regions engaged in diverse cognitive tasks (Duncan, 2010), exhibit FC only with MCC, 
mostly with its anterior part (Fig. 7), and not the ACC and PCC/retrosplenial. MCC is associated with cognitive and, mostly its posterior part exhibiting high $\mathrm{GABA}_{\mathrm{B}}$ densities resembling to motor regions, motor tasks (Beckman et al., 2009; Ridderinkhof et al., 2004; Pallomero-Galagher et al., 2009). The family formed from C1, C7-C9, which are linked to motor/occulomotor processes (see Results), exhibit FC only with the MCC, encompassing it almost entirely, including also its more posterior part (Fig. 7). Lastly, $\mathrm{C} 2$ and $\mathrm{C} 4$ exhibit FC with anterior MCC and the ACC (Fig. 7) that might relate to functions in the language domain.

Thus, the neural circuitry of each LFC family also encompasses distinct specialized cingulate divisions that correspond to the functional roles attributed to the LFC families (Duncan, 2010; Buckner, 2008; Barbas, 2000; Amunts et al., 2004b).

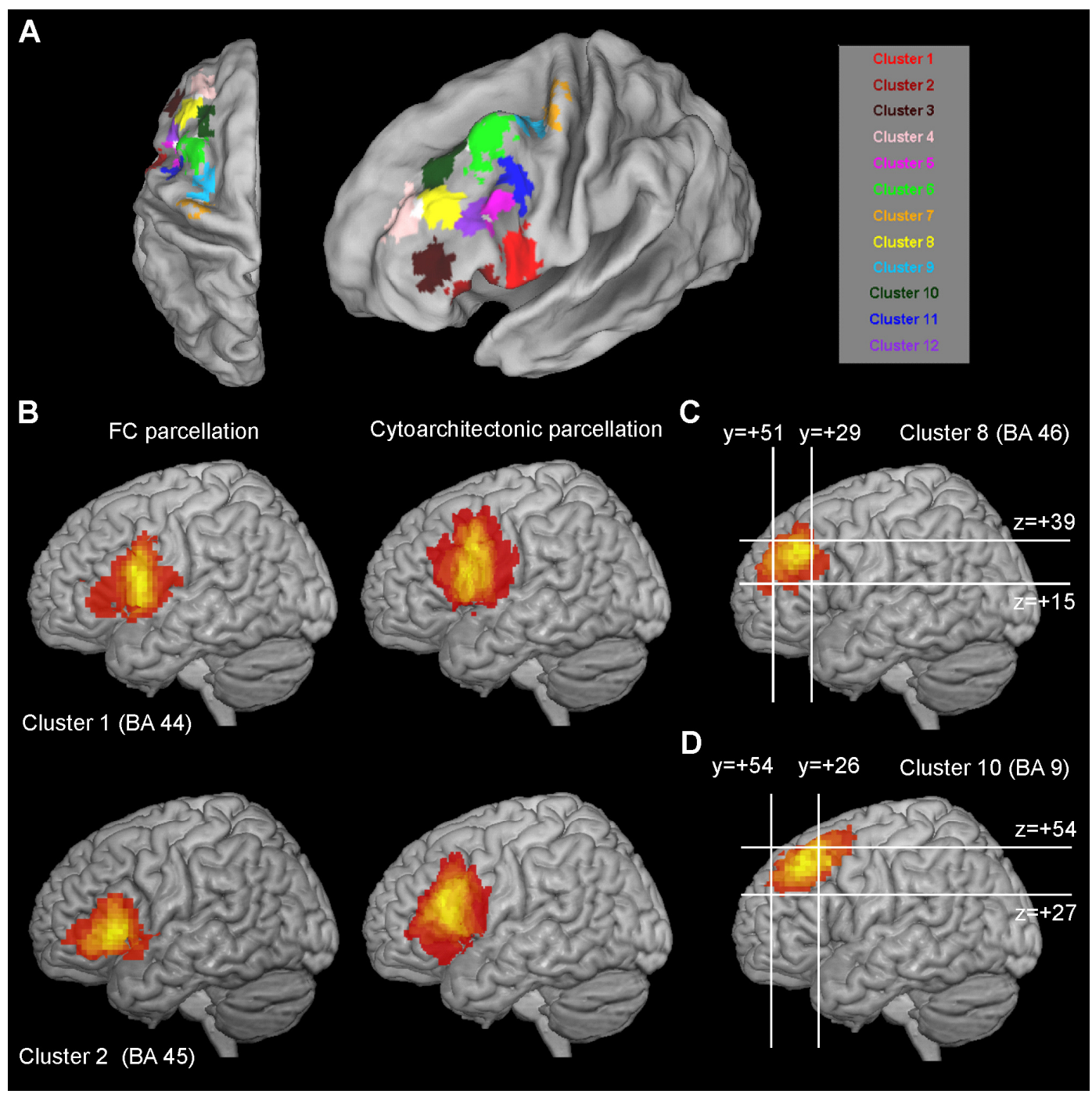

Figure 9. Variability maps of the clusters of modules across subjects. A. Cortical patches indicating the overlap of the clusters of modules across subjects. Each cortical patch denotes the extent of the variability (thresholded at $60 \%$ ) of each cluster of modules. The colour coding scheme is the same as the rest of the figures and white colour denotes intersection of cortical patches. B. Variability maps for C1 and C2 (putative BA 44 and 45 respectively). Variability maps of FC based parcellation (left column) are depicted along with variability maps from cytoarchitectonic based parcellation of putative BAs (right column). Cytoarchitectonic 
maps are part of the SPM anatomy toolbox (Eickhoff et al., 2005). Variability maps for C. C8 (putative BA 46) and D. C10 (putative (lateral) BA 9). Bounding boxes in panels C and D define conservative estimates of the location of BAs 46 and 9. These conservative estimates are derived from a cytoarchitectonic based parcellation (Rajkowska and Goldman-Rakic, 1995). Coordinates for the formation of the bounding boxes were approximated through a conversion from Tailarach to MNI space. Variability maps in panels B-D are unthresholded and bright yellow colours indicate higher overlap across subjects. Note the good correspondance between FC and cytoarchitectonic based parcellation.

Region 46 and motor control

Interestingly $\mathrm{C} 8$, i.e. presumed region 46 , formed a family with the most posterior LFC clusters. C8 exhibits FC with the SMA, preSMA, the ventral premotor cortex and the CMR (see Results, Fig. 6, 7) resembling connections among assumed macaque homologues (Lu et al., 1994; Goldman-Rakic, 1987; Barbas, 2000; Pickard and Strick, 2001). This connectivity pattern, along with additional functions of region 46 like the integration of goals maintained "on-line" and visuospatial information, can render possible the coordination of movement (Lu et al., 1994; Goldman-Rakic, 1987). The LFC family that C8 belongs to, its extensive and diffuse FC with premotor/occulomotor and visuospatial regions (Fig. 7) suggests that in the human brain there is a relative perseverance of the neural circuitry present in the monkey brain, involving region 46, wherein the latter seems involved in high levels of motor behaviour.

\section{Relation to LFC models}

The organization and FC of the LFC families along the anterior-posterior and dorsalventral axis partially comply with previous LFC models (Petrides, 2005): The dorsal family $(\mathrm{C} 6, \mathrm{C} 10)$ exhibits FC with the PCC/retrosplenial cortex through which access to memory related structures is accomplished (Fig. 7) (Morris et al., 1999). The ventral family $(\mathrm{C} 3, \mathrm{C} 5, \mathrm{C} 11, \mathrm{C} 12)$ is involved in a functional circuitry spanning audiovisual and somatomotor regions at the parietal and temporal cortex (Fig. 7). The posterior family (C1, C7-C9) includes regions involved in response selection and stimulus-response mappings (Badre and D'Esposito, 2009; Corbetta et al., 2008). However, members of this family are not constrained by Euclidean distance and despite that $\mathrm{C} 8$ is located at mid-dorsal LFC it does not exhibit similar whole-brain FC with its spatially adjacent subregions (Petrides, 2005). Instead it is affiliated with premotor regions, consistent with proposed LFC models (Miller and Cohen, 2001). The violation of a strict ordering according to Euclidean distance seemingly contradicts models advocating a hierarchical anterior-posterior gradient (Badre and D'Esposito, 2009). However, these models are compatible with the traced families since regions with a similar functional circuitry might be differentiated through their oscillation frequency (Baria et al., 2011) which is related to integrative capacities (Von Stein and Sarnthein, 2000) and/or the degree of rule abstraction and regulatory effective connectivity (Koechlin et al., 2003). Interestingly, the regions of the Koechlin model roughly coincide with the ones constituting a family (C1, C7-C9), with $\mathrm{C} 8$, also involved in high-order mechanisms of motor control, located more anterior than the rest, consistent with a potential hierarchical anterior-posterior gradient. A combination of rsfMRI-based parcellation and task-based paradigms can further inform the various LFC models. 


\section{Chapter 2}

Limitations and caveats

The resolution limit inherent in the module detection algorithm employed constraints the number of modules that can be resolved (Fortunato and Berthelemy, 2007). Consequently the modules obtained might be further subdivided. For instance, further subdivision of modules corresponding to presumed region 45 may reveal the anatomical subdivisions of this region (Amunts et al., 1999). Advances in network analysis might be employed to overcome the resolution limit (Ruan and Zhang, 2008). Moreover, the limited spatial resolution and preprocessing steps can lead to "signal bleeding" to adjacent regions. For instance, modules of $\mathrm{C} 1$ (region 44) can extend posterior to the prCS (Fig. 3 D) contrary to evidence from cytoarchitectonic studies. A parcellation that incorporates cortical distance information and higher acquisition resolution might ameliorate the results.

\section{Conclusions}

The present study unravels the intrinsic functional organization of the LFC. With rsfMRI we could trace neuroanatomically realistic LFC subregions and elucidate their distinct FC patterns that could segregate them into familes. This segregation seems related to functional specializations (Passingham et al., 2002) and reflects the view that the LFC is a constellation of specialized information processing systems (Miller, 2000). Similarities with known connectivity of assumed LFC monkey homologues were observed despite differences due to expansion and/or rewiring (Van Essen and Dierker, 2007; Semendeferi et al., 2002), extending and complying with recent evidence and LFC models (Kelly et al., 2010; Petrides et al., 2012; Petrides, 2005). Future comparative studies will offer a closer interspecies comparison. The current parcellation can guide and be complemented by other studies focusing on the LFC that could examine task-related properties and potential inter-hemispheric differences. Lastly, the methods used can be the basis for preoperative neurosurgery mapping.

\section{References}

Amodio DM, Frith CD (2006) Meeting of minds: the medial frontal cortex and social cognition. Nat Rev Neurosci 7:268-77.

Amunts K, Lenzen M, Friederici AD, Schleicher A, Morosan P, Palomero-Gallagher N, Zilles K (2010) Broca's region: novel organizational principles and multiple receptor mapping. PLoS Biol 8:e1000489.

Amunts K, Schleicher A, Burgel U, Mohlberg H, Uylings HBM, Zilles K (1999) Broca's region revisited: cytoarchitecture and intersubject variability J Comp Neurol 412:319-341.

Amunts K, Palomero-Gallagher N, Brass M, Derrfuss J, Zilles K, Cramon DYV (2004a) A receptor- and cytoarchitectonic correlate of the functionally defined inferior-frontal junction area. NeuroImage 22 (Suppl.), 50 .

Amunts K, Weiss PH, Mohlberg H, Pieperhoff P, Eickhoff S, Gurd JM, Marshall JC, Shah NJ, Fink GR, Zilles K (2004b) Analysis of neural mechanisms underlying verbal fluency in cytoarchitectonically defined stereotaxic space-the roles of Brodmann areas 44 and 45. NeuroImage 22:42-56.

Anwander A, Tittgemeyer M, Von Cramon DY, Friederici D, Knösche TR (2007) Connectivity-Based Parcellation of Broca's Area. Cereb Cortex 17:816-25. 
Asplund CL, Todd JJ, Snyder AP, Marois R (2010) A central role for the lateral prefrontal cortex in goaldirected and stimulus-driven attention. Nat Neurosci 13:507-12.

Astafiev SV, Shulman GL, Stanley CM, Snyder AZ, Essen DC Van, Corbetta M (2003) Functional organization of human intraparietal and frontal cortex for attending, looking, and pointing. J Neurosci 23:4689-99.

Badre D, D'Esposito M (2009) Is the rostro-caudal axis of the frontal lobe hierarchical? Nat Rev Neurosci 10:659-69.

Barbas H (2000) Proceedings of the Human Cerebral Cortex: From Gene to Structure and Function Connections underlying the synthesis of cognition, memory, and emotion in primate prefrontal cortices. Brain Res 52:319 -330.

Baria AT, Baliki MN, Parrish T, Apkarian V (2011) Anatomical and Functional Assemblies of Brain BOLD Oscillations. J Neurosci 31:7910-9.

Barnes KA, Cohen AL, Power JD, Nelson SM, Dosenbach YBL, Miezin FM, Petersen SE, Schlaggar BL (2010) Identifying Basal Ganglia divisions in individuals using resting-state functional connectivity MRI. Frontiers in Systems Neuroscience 4:18.

Barnes KA, Nelson SM, Cohen AL, Power JD, Coalson RS, Miezin FM, Vogel AC, Dubis JW, Church J a, Petersen SE, Schlaggar BL (2011) Parcellation in Left Lateral Parietal Cortex Is Similar in Adults and Children. Cereb Cortex 1-11.

Beckmann M, Johansen-Berg H, Rushworth MFS (2009) Connectivity-based parcellation of human cingulate cortex and its relation to functional specialization. J Neurosci 29:1175-90.

Blondel VD, Guillaume JL, Lefebvre E (2008) Fast unfolding of communities in large networks. J. Stat. Mech. P10008.

Blanke O, Spinelli L, Michel CM, Thut G, Landis T, Seeck M (2000) Location of the human frontal eye field as defined by electrical cortical stimulation: anatomical, functional andelectrophysiological characteristics. Neuroreport 11:1907-1913

Brass M, Derrfuss J, Forstmann B, Crammon YD von (2005) The role of the inferior frontal junction area in cognitive control. Trends Cogn Sci 9:314-316.

Brodmann, K. (1909) Vergleichende Localisationslehre der Grosshirnrinde in ihren rinzipien dargestellt auf Grund des Zellenbaues. Leipzig: Barth.

Buckner RL, Andrews-Hanna JR, Schacter DL (2008) The brain's default network: anatomy, function, and relevance to disease. Ann NY Acad Sci 1124:1-38.

Cohen AL, Fair D a, Dosenbach NUF, Miezin FM, Dierker D, Essen DC Van, Schlaggar BL, Petersen SE (2008) Defining functional areas in individual human brains using resting functional connectivity MRI. NeuroImage 41:45-57.

Corbetta M, Patel G, Shulman GL (2008) The reorienting system of the human brain: from environment to theory of mind. Neuron 58:306-24.

Cordes D, Haughton VM, Arfanakis K, Wendt GJ, Turski P, Moritz CH, Quigley M, Meyerand ME (2000) Mapping functionally related regions of brain with functional connectivity MR imaging. Americ J Neuroradio 21:1636-44. 


\section{Chapter 2}

De Luca M, Beckmann CF, Stefano N De, Matthews PM, Smith SM (2006) fMRI resting state networks define distinct modes of long-distance interactions in the human brain. NeuroImage 29:1359-67.

Doucet G, Naveau M, Petit L, Delcroix N, Zago L, Crivello F, Jobard G, Tzourio-Mazoyer N, Mazoyer B, Mellet E, Joliot M (2011) Brain activity at rest: A multi-scale hierarchical functional organization. J Neurophysiol. 105:2753-63.

Duncan J (2010) The multiple-demand (MD) system of the primate brain: mental programs for intelligent behaviour. Trends Cogn Sci 14:172-9.

Duncan J, Owen M (2000) Common regions of the human frontal lobe recruited by diverse cognitive demands. Trends Neurosci 23:475-83.

Eickhoff SB, Stephan KE, Mohlberg H, Grefkes C, Fink GR, Amunts K, Zilles K (2005) A new SPM toolbox for combining probabilistic cytoarchitectonic maps and functional imaging data. NeuroImage 25:1325 - 1335.

Esposito F, Scarabino T, Hyvarinen A, Himberg J, Formisano E, Comani S, Tedeschi G, Goebel R, Seifritz E, Salle F Di (2005) Independent component analysis of fMRI group studies by self-organizing clustering. NeuroImage 25:193-205.

Fischl B, Rajendran N, Busa E, Augustinack J, Hinds O, Yeo BTT, Mohlberg H, Amunts K, Zilles K (2008) Cortical folding patterns and predicting cytoarchitecture. Cereb Cortex 18:1973-80.

Ford A, McGregor KM, Case K, Crosson B, White KD (2010) Structural connectivity of Broca's area and medial frontal cortex. NeuroImage 52:1230-7.

Fox MD, Corbetta M, Snyder AZ, Vincent JL, Raichle ME (2006) Spontaneous neuronal activity distinguishes human dorsal and ventral attention systems. Proc Natl Acad Sci USA 103:10046-51.

Fortunato S (2010) Community detection in graphs. Phys Rep 486:75-174.

Fortunato S, Barthélemy M (2007) Resolution limit in community detection. Proc Natl Acad Sci U S A 104:36-41.

Frey S, Campbell JSW, Pike GB, Petrides M (2008) Dissociating the human language pathways with high angular resolution diffusion fiber tractography. J Neurosci 28:11435-44.

Gerbella M, Belmalih A, Borra E, Rozzi S, Luppino G (2010) Cortical connections of the macaque caudal ventrolateral prefrontal areas 45A and 45B. Cereb Cortex 20:141-68.

Geyer S (2004) The microstructural border between the motor and the cognitive domain in the human cerebral cortex. Advances in anatomy embryology and cell biology, vol 174. Springer, Berlin.

Goldman-Rakic PS (1987) Motor control function of the prefrontal cortex. In: Motor areas of the cerebral cortex Wiley, Chichester (Ciba Foundation Symposium 132) pp187-200.

Goldman-Rakic PS (1996) The prefrontal landscape: implications of functional architecture for understanding human mentation and the central executive. Philos Trans R Soc Lond B Biol Sci 351:1445-53.

Good BH, Montjoye YA De, Clauset A (2010) The performance of modularity maximization in practical contexts Phys. Rev. E 81, 046106.

Guimera R (2005) Functional cartography of complex metabolic networks. Nature 433:895-900. 
Hagler DJ, Riecke L, Sereno MI (2007) Parietal and superior frontal visuospatial maps activated by pointing and saccades. NeuroImage 35:1562-77.

Honey CJ, Kötter R, Breakspear M, Sporns O (2007) Network structure of cerebral cortex shapes functional connectivity on multiple time scales. Proc Natl Acad Sci USA 104:10240-5.

Honey CJ, Sporns O, Cammoun L, Gigandet X, Thiran JP, Meuli R, Hagmann P (2009) Predicting human resting-state functional connectivity from structural connectivity. Proc Natl Acad Sci USA 106:2035-40.

Hutchison RM, Womelsdorf T, Gati JS, Leung LS, Menon RS, Everling S (2012) Resting-state connectivity identifies distinct functional networks in macaque cingulate cortex. Cereb Cortex 22:1294-1308.

Iturria-Medina Y, Pérez Fernández A, Morris DM, Canales-Rodríguez EJ, Haroon HA, García Pentón L, Augath M, Galán García L, Logothetis N, Parker GJM, Melie-García L (2011) Brain hemispheric structural efficiency and interconnectivity rightward asymmetry in human and nonhuman primates. Cereb Cortex 21:5667.

Karrer B, Levina E, Newman MEJ (2008) Robustness of community structure in networks. Phys Rev E 77:19.

Kelly C, Uddin LQ, Shehzad Z, Margulies DS, Castellanos FX, Milham MP, Petrides M (2010) Broca's region: linking human brain functional connectivity data and non-human primate tracing anatomy studies. Eur J Neurosci 32:383-98.

Kim J-H, Lee J-M, Jo HJ, Kim SH, Lee JH, Kim ST, Seo SW, Cox RW, Na DL, Kim SI, Saad ZS (2010) Defining functional SMA and pre-SMA subregions in human MFC using resting state fMRI: functional connectivity-based parcellation method. NeuroImage 49:2375-86.

Klein JC, Behrens TEJ, Robson MD, Mackay CE, Higham DJ, Johansen-Berg H (2007) Connectivity-based parcellation of human cortex using diffusion MRI: Establishing reproducibility, validity and observer independence in BA 44/45 and SMA/pre-SMA. NeuroImage 34:204-11.

Koechlin E, Ody C, Kouneiher F (2003) The architecture of cognitive control in the human prefrontal cortex. Science 302:1181-5.

Koyama M, Hasegawa I, Osada T, Adachi Y, Nakahara K, Miyashita Y (2004) Functional magnetic resonance imaging of macaque monkeys performing visually guided saccade tasks: comparison of cortical eye fields with humans. Neuron 41:795-807.

Lancichinetti A, Fortunato S (2009) Community detection algorithms: a comparative analysis. Phys Rev E 80, 056117.

Lancichinetti A, Radicchi F, Ramasco JJ (2010) Statistical significance of communities in networks. Phys Rev E 81:046110.

Liang Z, King J, Zhang N (2011) Uncovering intrinsic connectional architecture of functional networks in awake rat brain. J Neurosci 31:3776-83.

Lu MT, Preston JB, Strick PL (1994) Interconnections between the prefrontal cortex and the premotor areas in the frontal lobes. J Comp Neurol 341:375-392. 


\section{Chapter 2}

Margulies DS, Vincent JL, Kelly C, Lohmann G, Uddin LQ, Biswal BB, Villringer A, Castellanos FX, Milham MP, Petrides M (2009) Precuneus shares intrinsic functional architecture in humans and monkeys. Proc Natl Acad Sci USA 106:20069-74.

Meila M (2007) Comparing clusterings—an information based distance. J Multivar Analysis 98:873-895.

Meunier D, Lambiotte R, Fornito A, Ersche KD, Bullmore ET (2009) Hierarchical modularity in human brain functional networks Front Neuroinformatics 3:1-12.

Miller EK (2000) The prefrontal cortex and cognitive control. Nat Rev Neurosci 1:59-65.

Miller EK, Cohen JD (2001) An integrative theory of prefrontal cortex function. Ann Rev Neurosci 24:167202.

Miranda M (2003) Role of cholinergic system on the construction of memories: Taste memory encoding. Neurobiology of Learning and Memory 80:211-222.

Morris R, Petrides M, Pandya DN (1999) Architecture and connections of retrosplenial area 30 in the rhesus monkey (macaca mulatta). Eur J Neurosci 11:2506-2518.

Nelson SM, Cohen AL, Power JD, Wig GS, Miezin FM, Wheeler ME, Velanova K, Donaldson DI, Phillips JS, Schlaggar BL, Petersen SE (2010) A parcellation scheme for human left lateral parietal cortex. Neuron 67:156-70.

Newman MEJ (2006) Modularity and community structure in networks. Proc Natl Acad Sci USA 103:857782 .

Palomero-Gallagher N, Vogt B a, Schleicher A, Mayberg HS, Zilles K (2009) Receptor architecture of human cingulate cortex: evaluation of the four-region neurobiological model. Hum Brain Mapp 30:2336-55.

Pandya DN, Yeterian EH (1996) Comparison of prefrontal architecture and connections. Philos Trans R Soc Lond B Biol Sci 351:1423-32.

Passingham RE, Stephan KE, Kötter R (2002) The anatomical basis of functional localization in the cortex. Nat Rev Neurosci 3:606-16.

Paus T (2001) Primate anterior cingulate cortex: where motor control, drive and cognition interface. Nat Rev Neurosci 2:417-24.

Petrides M, Pandya, DN (1994) Comparative architectonic analysis of the human and the macaque frontal cortex. In Handbook of neuropsychology (F. Boller J. Grafman eds), vol. 9, pp. 17-58. Amsterdam: Elsevier

Petrides M, Pandya D.N. (2004) The frontal cortex. In: The human nervous system. 2nd edition. (Paxinos G, Mai J.K, ed) pp. 950-972. Elsevier Academic Press San Diego.

Petrides M (2005) Lateral prefrontal cortex: architectonic and functional organization. Philos Trans R Soc Lond B Biol Sci 360:781-95.

Petrides M, Cadoret G, Mackey S (2005) Orofacial somatomotor responses in the macaque monkey homologue of Broca's area. Nature 435:1235-8. 
Petrides M, Pandya DN (2009) Distinct parietal and temporal pathways to the homologues of Broca's area in the monkey. PLoS Biology 7:e1000170.

Petrides M, Tomaiuolo F, Yeterian EH, Pandya DN (2012) The Prefrontal Cortex: Comparative Architectonic Organization in the Human and the Macaque Monkey Brains. Cortex 48:46 -57.

Picard N, Strick PL (2001) Imaging the premotor areas. Curr Opin Neurobiol 11:663-72.

Rajkowska G, Goldman-Rakic PS (1995) Cytoarchitectonic definition of prefrontal areas in the normal human cortex: II Variability in locations of areas 9 and 46 and relationship to the Tailarach coordinate system. Cereb Cortex 5:323-337.

Raichle ME, MacLeod M, Snyder Z, Powers WJ, Gusnard D, Shulman GL (2001) A default mode of brain function. Proc Natl Acad Sci USA 98:676-82.

Ridderinkhof KR, Ullsperger M, Crone EA, Nieuwenhuis S (2004) The Role of the Medial Frontal Cortex in Cognitive Control. Science 306:443-447.

Ruan J, Zhang W (2008) Identifying network communities with a high resolution. Phys. Rev. E 77:016104.

Rubinov M, Sporns O (2010) Complex network measures of brain connectivity: uses and interpretations. NeuroImage 52:1059-69.

Rubinov M, Sporns O (2011) Weight-conserving characterization of complex functional brain networks. NeuroImage 56:2068-79.

Sarkissov SA, Filimonoff I.N, Kononowa EP, Preobraschenskaja IS, Kukuew LA (1955) Atlas of the cytoarchitectonics of the human cerebral cortex. Moscow:Medgiz.

Semendeferi K, Lu A, Schenker N, Damasio H (2002) Humans and great apes share a large frontal cortex. Nat Neurosci 5:272-6.

Shen X, Papademetris X, Constable RT (2010) Graph-theory based parcellation of functional subunits in the brain from resting-state fMRI data. NeuroImage 50:1027-35.

Sporns O, Honey CJ, Kötter R (2007) Identification and Classification of Hubs in Brain Networks. PLoS ONE 2(10): e1049.

Stiers P, Mennes M, Sunaert S (2010) Distributed task coding throughout the multiple demand network of the human frontal-insular cortex. NeuroImage 52:252-62.

Tian JR, Lynch JC (1996) Corticocortical input to the smooth and saccadic eye movement subregions of the frontal eye field in Cebus monkeys. J Neurophys 76:2754-7.

Uylings HBM, Groenewegen HJ, Kolb B (2003) Do rats have a prefrontal cortex? Behavioural Brain Res 146:3-17.

Uylings HBM, Rajkowska G, Sanz-Arigita E, Amunts K, Zilles K (2005) Consequences of large interindividual variability for human brain atlases: converging macroscopical imaging and microscopical neuroanatomy. Anat and Embryol 210:423-31. 


\section{Chapter 2}

Uylings HBM, Sanz-Arigita EJ, Vos K de, Pool CW, Evers P, Rajkowska G (2010) 3-D cytoarchitectonic parcellation of human orbitofrontal cortex correlation with postmortem MRI. Psych Res 183:1-20.

Van Dijk KR, Hedden T, Venkataraman A, Evans KC, Lazar SW, Buckner RL (2010) Intrinsic functional connectivity as a tool for human connectomics: theory, properties, and optimization. J Neurophys 103:297321.

Van Essen DC, Dierker DL (2007) Surface-based and probabilistic atlases of primate cerebral cortex. Neuron 56:209-25.

Vincent JL, Kahn I, Snyder AZ, Raichle ME, Buckner RL (2008) Evidence for a frontoparietal control system revealed by intrinsic functional connectivity. J Neurophys100:3328-42.

Von Stein A, Sarnthein J (2000) Different frequencies for different scales of cortical integration: from local gamma to long range alpha/theta synchronization. Intern J Psychophysiol 38:301-13.

Wilson CRE, Gaffan D, Browning PGF, Baxter MG (2010) Functional localization within the prefrontal cortex: missing the forest for the trees? Trends Neurosci 33:533-40.

Yeo BTT, Krienen FM, Sepulcre J, Sabuncu MR, Lashkari D, Hollinshead M, Roffman JL, Smoller JW, Zollei L, Polimeni JR, Fischl B, Liu H, Buckner RL (2011) The Organization of the Human Cerebral Cortex Estimated By Functional Connectivity. J Neurophysio.

Yeterian EH, Pandya DN, Tomaiuolo F, Petrides M (2012) The cortical connectivity of the prefrontal cortex in the monkey brain. Cortex 48:58 -81.

Zhang D, Snyder AZ, Shimony JS, Fox MD, Raichle ME (2010) Noninvasive functional and structural connectivity mapping of the human thalamocortical system. Cereb Cortex 20:1187-94.

Zilles K, Amunts K (2010) Centenary of Brodmann's map--conception and fate. Nat Rev Neurosci 11:139-45 


\section{Chapter 3}

\section{Mapping the hierarchical layout of the structural network of the macaque prefrontal cortex}

Goulas A, Uylings HBM, Stiers P (in press) Mapping the hierarchical layout of the structural network of the macaque prefrontal cortex. Cereb Cortex. 


\begin{abstract}
A consensus on the prefrontal cortex (PFC) holds that it is pivotal for flexible behaviour and the integration of the cognitive, affective, and motivational domains. Certain models have been put forth and a dominant model postulates a hierarchical anteriorposterior gradient. The structural connectivity principles of this model dictate that increasingly anterior PFC regions exhibit more efferent connections toward posterior ones than vice versa. Such hierarchical asymmetry principles are thought to pertain to the macaque PFC. Additionally, the laminar patterns of connectivity of PFC regions can be used for defining hierarchies. In the current study, we formally tested the asymmetry based hierarchical principles of the anterior-posterior model by employing an exhaustive dataset on macaque PFC connectivity and tools from network science. On the one hand, the asymmetry based principles and predictions of the hierarchical anterior-posterior model were not confirmed. The wiring of the macaque PFC does not fully correspond to the principles of the model and its asymmetry based hierarchical layout does not follow a strict anterior-posterior gradient. On the other hand, our results suggest that the laminar based hierarchy seems a more tenable working hypothesis for models advocating an anterior-posterior gradient. Our results can inform models of the human PFC.
\end{abstract}




\section{$\underline{\text { Introduction }}$}

The prefrontal cortex (PFC) is associated with flexible and goal directed behaviour, integrating information from various modalities and bridging the cognitive, affective, and motivational domain (Barbas, 2000; Groenewegen and Uylings, 2000; Miller and Cohen, 2001; Kouneiher et al., 2009). The PFC, as the rest of the brain, can be conceived as a complex system with its distinct subregions interacting through their efferent and afferent structural connections. Such a conceptualization, along with the employment of network analysis, has been previously used to elucidate organization principles of the PFC (Kötter et al., 2001), the visual system (Hilgetag et al., 2000) and the large scale brain network of the macaque (Modha and Singh, 2010). In order to understand the organization of a complex system the concept of hierarchy is often employed. In general terms, "hierarchy" describes the relation among a set of elements indicating which element lies "below" or "above" another (Bond, 2004). The concept of hierarchy and appropriate analytic tools have been used for instance in systems biology to elucidate regulatory pathways in protein interaction networks (Ispolatov and Maslov, 2008).

In neuroscience, the concept of hierarchy has been employed in order to examine the structural architecture of the visual system of the macaque (Felleman and Van Essen, 1991). The principles of this hierarchical layout are based on the laminar patterns of connections between the regions constituting the visual system. The laminar origin and termination of a connection is used in order to classify a connection as "feedforward" (FF), "feedback" (FB) and "lateral". FF connections are efferents from regions lower in the hierarchy, e.g. V1, towards regions higher in the hierarchy, e.g. V4. FB connections follow the reverse order and lateral connections link regions at approximately the same level within the visual processing architecture. Several computational tools have been fruitfully adopted in order to find the hierarchical arrangement of the regions of the visual system (Hilgetag et al., 1996). Similar hierarchical principles seem to also hold for the somatosensory and motor cortices (Felleman and Van Essen, 1991).

The definition of hierarchy based on laminar patterns of connections is a popular concept mainly applied to the visual system. Other definitions of hierarchy have been employed in order to formulate principles and models of the PFC. One such model postulates a hierarchical anterior-posterior gradient (Badre and D'Esposito, 2009). This model is the culmination of theories and evidence on the hierarchical nature of the PFC build primarily on task-based studies (Koechlin et al., 2003; Koechlin and Summerfield, 2007). Task-based fMRI paradigms involving humans revealed that increasingly abstract rules engage more anterior regions (Badre and D'Esposito, 2007; Koechlin et al., 2003). With such function-related findings as a starting point, additional PFC features such as the structural connections of the PFC of the macaque have been suggested to support a hierarchical anterior-posterior gradient (Badre and D'Esposito, 2009). According to the hierarchical anterior-posterior model the structural connectivity of the PFC adheres to the principles of contiguity and asymmetry. We shall refer to this type of hierarchy as asymmetry based hierarchy $(\mathrm{ABH})$. The asymmetry principle suggests that regions higher in the hierarchy exhibit a "surplus" of efferents, i.e. more connections, to regions lower in the hierarchy. The contiguity principle dictates that spatially adjacent regions can exhibit bidirectional connections. The principle of contiguity is likely adopted in order to accommodate the fact that excessive reciprocal connections exist between adjacent, i.e. spatially contiguous, PFC regions (Koechlin and Summerfield, 2007; Badre and D'Esposito, 2009). Such reciprocicity and decrease 


\section{Chapter 3}

of connectivity between PFC regions with increased spatial distance, i.e. contiguous or separated by other regions, is demonstrated in computational analysis of the connectivity of the macaque PFC (Kötter and Stephan, 2003; Averbeck et al., 2008).

The hierarchical anterior-posterior model postulates that region 10 , i.e. the most anterior region, lies at the top of the hierarchy, while posterior regions, like region 8 , lie at the bottom of the hierarchy. This is inferred from the observation that there are more efferent connections from anterior to posterior regions than vice versa. In other words, according to the model, the dominant direction of the PFC network obeys an anteriorposterior gradient. Consequently, anterior regions are favoured to have a "regulatory" role by broadcasting signals to more posterior regions and thus lie higher in the hierarchy. In terms of control theory, regions high in such a hierarchy function as broadcasters, and thus are more ideally positioned for influencing other regions than getting influenced by other regions, while regions low in the hierarchy function as receivers, exhibiting the reverse properties (Hilgetag et al., 2002; Kötter et al., 2001).

The hierarchical anterior-posterior model is characterized by some limitations/open issues: Only data from a couple of tracing studies were used (data limitation). Moreover, a descriptive approach was adopted and no formal analyses were employed (quantification limitation). Furthermore, there is no clear consensus about which exact regions should be selected for a subsequent examination of their structural connectivity. The various hierarchical models, employing task-based fMRI paradigms, involve a partially overlapping but not identical set of regions engaged by experimental manipulations (Koechlin al., 2003; Badre and D'Esposito, 2007), and due to excessive inter-subject variability (Uylings et al., 2005), no clear unique anatomical substrate can be attributed to them. Additionally, the aforementioned experimental manipulations involved human subjects. Hence, an extrapolation from the human to the macaque PFC can introduce further inaccuracies (localization limitation).

In the current study we will test the principles and predictions of the hierarchical anterior-posterior model concerning the structural connectivity of the macaque PFC, in a formal way and by employing an exhaustive dataset. We should stress that we chose to base our analysis on the principles of the hierarchical anterior-posterior model, i.e. asymmetry and contiguity, and not some other hierarchical principles for the very reason that such principles were put forth in (Badre and D'Esposito, 2009). Hence, the core analyses employed in our study are tailored for the evaluation of the hierarchical anterior-posterior model and aim at mapping the $\mathrm{ABH}$ layout of the PFC. In order to unravel the $\mathrm{ABH}$ layout of the macaque PFC we employ an algorithm previously used in systems biology (Ispolatov and Maslov, 2008). Moreover the data currently used represent a manifold of tracing studies collated in the CoCoMac neuroinformatics database (Kötter, 2004) and constitute our current best knowledge on macaque connectivity. The localization limitation in conjunction with studies pinpointing a holistic approach for the understanding of the PFC (Barbas, 1995, 2000; Kouneiher et al., 2009; Taren et al., 2011) led us to investigate the PFC as a whole. This approach also makes plausible the investigation of a possible anterior-posterior hierarchical gradient in the medial and orbital parts of the PFC (O'Reilly, 2010).

As a complementary analysis, we aimed to investigate how the aforementioned $\mathrm{ABH}$ layout corresponds to a hierarchical layout of the PFC based on a different concept and principles, i.e. the laminar patterns of connections of the PFC regions (Barbas and Rempel-Clower, 1997). Within this schema, PFC connections can be termed as FB and FF in analogy with the patterns observed in the visual system. Hence, the laminar patterns of the connections can be used for defining the hierarchical arrangement of the 
PFC regions. This analysis, that we shall refer to as laminar based hierarchy (LBH), will elucidate if and how the two different hierarchical concepts and principles are related and examine if an anterior-posterior hierarchical gradient is tenable by either definitions of hierarchy.

\section{Materials and Methods}

Datasets

The connectivity of the macaque PFC is represented as an $\mathrm{NxN}$ directed graph $\mathrm{G}(\mathrm{N}$, $\mathrm{E}$ ), where $\mathrm{N}$ represents the number of $\mathrm{PFC}$ regions and $\mathrm{E}$ the edges (or arcs) in between them. Hence, a directed connection from region $i$ to region $j$ is represented with an entry $\mathrm{G}(\mathrm{i}, \mathrm{j})$. The connectivity matrix used in the current study is a binary version of the connectivity matrix used in (Kötter et al., 2001). Therefore, entries $G(i, j)$ can be 1 , if a connection exists, or 0 , if a connection is absent. These connectivity data were collated from 148 tracing studies available in the CoCoMac database (Kötter, 2004) and mapped to Walker's parcellation scheme (Walker, 1940) with Objective Relational Transformation. This technique permits assembling data from different cortical parcellations in one "reference" parcellation scheme. Such transformations are based on spatial relations between regions from different parcellations schemes, e.g. region A from parcellation $\mathrm{X}$ is identical to region $\mathrm{B}$ from parcellation $\mathrm{Y}$, and dedicated algorithms and algebra that have been developed for this purpose (Stephan et al., 2000, 2001). We shall refer to the first dataset as "Walker 1" (Table 1, Fig. 1 A). Walker's parcellation scheme is coarse but it allows the gathering of a vast number of data, it is largely compatible with other parcellation schemes and still widely used (Kötter et al., 2001, 2007). Moreover, the adoption of the aforementioned dataset allows results derived from the current study to be combined with insights from previous studies (Kötter et al., 2001; Kötter and Stephan, 2003). The connectivity matrix in (Kötter et al., 2001) contains entries/connections of unknown status which were treated as absent. Since the assumption that connections of unknown status are absent ones can bias the results (Kötter and Stephan, 2003) we will also use an updated connectivity dataset based on Walker's parcellation scheme (Kötter et al., 2007) that we will refer to as "Walker 2" (Table 1, Fig. 1 B). In the later dataset only one connection is of unknown status and was treated as absent. Additionally, in order to examine the influence that the adoption of a particular parcellation scheme might have, we will use data on PFC connectivity as presented in a recent network analysis of the data collated in the CoCoMac database involving more than 400 tracing studies (Modha and Singh, 2010). We shall refer to this connectivity dataset as "Modha and Singh PFC". We should note that we treated this dataset "as is", i.e. as presented by the authors of the later study: no further regions, e.g. cingulate region 24 (see Uylings and Van Eden, 1990), were added as part of the PFC. The usage of different datasets and/or parcellation schemes allows us to assess their impact on the results and conclusions of the analyses.

\section{Problem formulation}

An $\mathrm{ABH}$ organization is characterized by the principles of asymmetry and contiguity dictating that bidirectional connections are allowed between contiguous regions, while for non-contiguous ones an asymmetry should hold. In other words, asymmetry dictates that more efferent connections exist from regions higher in the hierarchy towards 


\section{Chapter 3}

regions lower in the hierarchy than vice versa (Fig. 2). Hence, the dominant direction within the structural network obeys an anterior-posterior gradient. In order to formulate the problem at hand in a rigorous way, we represent the connectivity of the PFC as a directed graph $G(N, E)$ as previously described. Hence, the problem of $A B H$ arrangement of the PFC regions can be formulated as converting the graph to an acyclic one, i.e. with no paths that start and end at the same node, in such a way that there is a minimum deletion of "anti-hierarchical" arcs/connections that form loops/cycles (Ispolatov and Maslov, 2008).

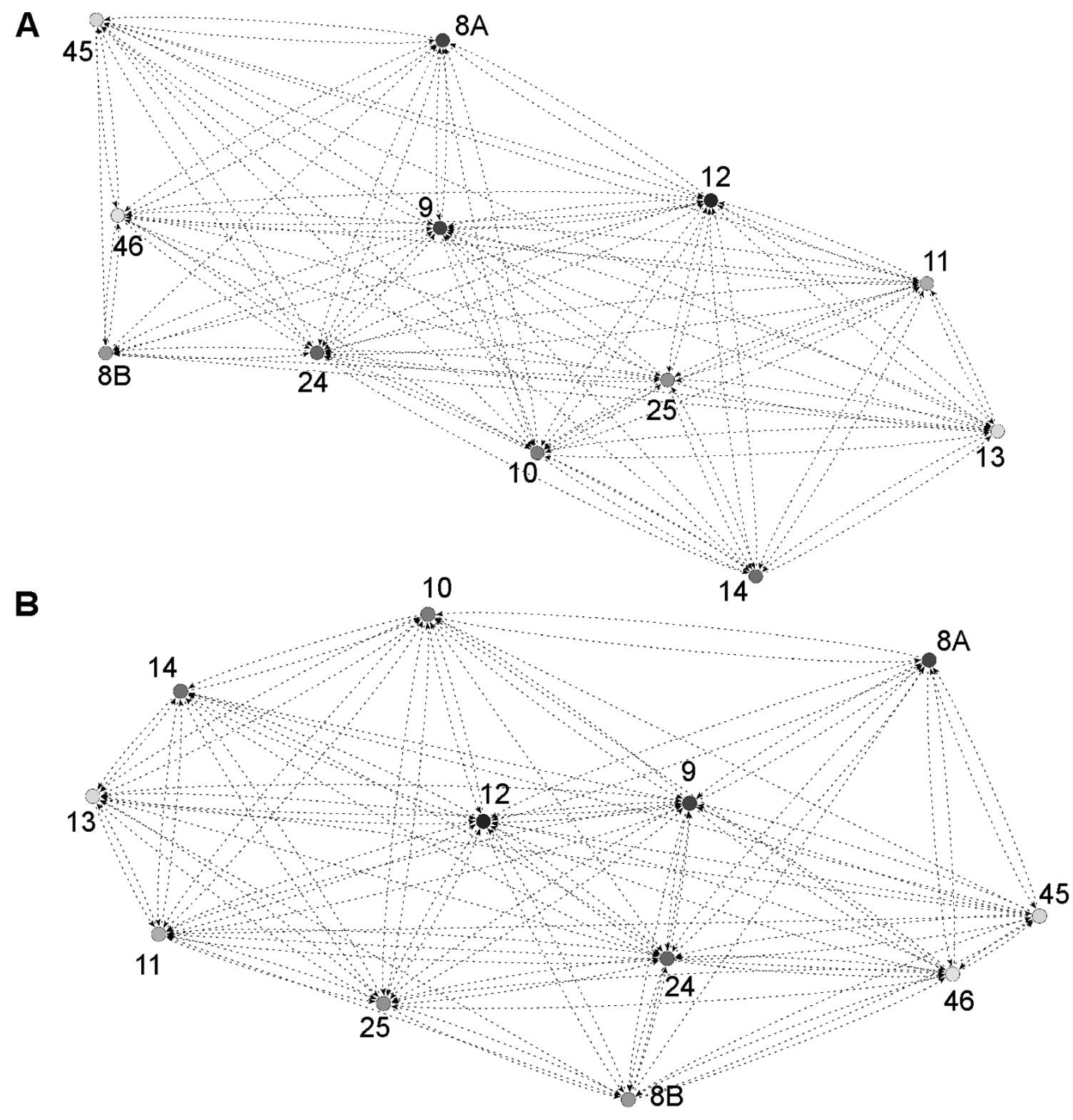

Figure 1. Graph representation of the PFC connectivity datasets. The PFC connectivity based on Walker's parcellation scheme is represented as a directed graph. Dataset A. Walker 1 and B. Walker 2. The directed graphs are arranged with the Kamada-Kawai spring embedding algorithm. 
This problem is known in graph theory as the minimum feedback arc set problem (e.g. Eades et al., 1993). Such approaches have been employed for the analysis of complex networks in systems biology in order to produce a hierarchical layout and trace nodes functioning as regulators and targets (Ispolatov and Maslov, 2008). Other recent applications include the analysis of large-scale biological systems, i.e. gene regulatory networks (Soranzo et al., 2012). Similar concepts and the appropriate network metrics have been employed for unravelling PFC regions that are anatomically embedded in such way to predominantly influence rather than get influenced by other regions (Kötter et al., 2001). While the primate cortex is clearly not "naturally" acyclic, e.g. it possesses a high clustering coefficient (e.g. Sporns et al., 2007), and consequently the existence of "anti-hierarchical" arcs/connections is certain, its conversion to an acyclic graph with the minimal possible arc deletion will allow us to reveal an hierarchical arrangement of the PFC regions (see below). We should note that such connections should not be considered as "redundant", since their absence would not correspond to a neuroanatomically realistic network. Instead they should be conceived as connections opposing the dominant direction within the macaque PFC network.

Table 1. Summary of basic features of the datasets used.

\begin{tabular}{|l|r|r|r|r|}
\hline & $\begin{array}{c}\text { Nr of } \\
\text { nodes }\end{array}$ & \multicolumn{1}{|c|}{$\begin{array}{c}\text { Nr of } \\
\text { connections }\end{array}$} & $\begin{array}{c}\text { Edge } \\
\text { density }\end{array}$ & $\begin{array}{c}\text { Nr of connections of } \\
\text { unknown status (treated } \\
\text { as absent) }\end{array}$ \\
\hline Walker 1 & 12 & 97 & 0.73 & \\
\hline Walker 2 & 12 & 101 & 0.76 & \\
\hline
\end{tabular}

Basic network features for the main datasets used. While the number of nodes is the same for both datasets, the number of connections is different. This stems from the fact that Walker 2 is an updated connectivity matrix where information about the connections with a previous unknown status has been incorporated. Note that while Walker 1 has 8 connections with unknown status, Walker 2 has only 1 . In both cases such connections were treated as absent. Edge density is the number of existing connections divided by the number of all possible connections in each dataset.

In order to hierarchically arrange the PFC regions we adopt a probabilistic approach based on simulated annealing that has shown good performance in synthetic and natural graphs (Ispolatov and Maslov, 2008). We shall refer to this procedure as ABH optimization. Below we give a brief description of the various steps of the algorithm (for details see Ispolatov and Maslov, 2008).

\section{Optimization procedure}

Simulated annealing is a stochastic procedure for the approximation of global optima (Metropolis et al., 1953; Kirkpatrick et al., 1983). In our case the global optimum is an arrangement of the PFC connectivity, represented as a graph $\mathrm{G}(\mathrm{N}, \mathrm{E})$, in such a way that an acyclic graph is produced with the fewest possible removals of "antihierarchical" arcs/connections. To this end, the $\mathrm{N}$ nodes of the graph assembled from CoCoMac data are assigned to $M$ levels randomly, where $M=2,3, \ldots N$. Higher numbers indicate levels higher in the hierarchy. The sum of the connections from nodes placed at low hierarchical levels to nodes in higher or equal levels are declared "anti-hierarchical" 


\section{Chapter 3}

arcs/connections, and constitute the cost, or energy (En), of the system. Thus the goal is to arrange the nodes in a way that would result in the minimization of En (eq. 1 and 2).

During the optimization procedure two different cost functions, which quantify the energy of the system, are minimized: 1) Sum of "anti-hierarchical" connections (Fig. 2 B) (eq. 1):

$$
E n=\sum_{i \rightarrow j}\left[H\left(m_{j}-m_{i}\right)\right]
$$

where $i \rightarrow j$ denotes a structural directed connection (or arc/edge) from node (region) $\mathrm{i}$ to node $\mathrm{j}, \mathrm{m}_{\mathrm{i}}$ and $\mathrm{m}_{\mathrm{j}}$ is the hierarchical level of node $\mathrm{i}$ and $\mathrm{j}$, and $\mathrm{H}$ is the discrete Heaviside step function. 2) Sum of "anti-hierarchical" connections that involve noncontiguous regions (Fig. 2 C) (eq. 2):

$$
E n=\sum_{i \rightarrow j}\left[H\left(m_{j}-m_{i}\right) \cdot \delta_{i, j}\right]
$$

where:

$$
\delta_{i, j}=\left\{\begin{array}{l}
1, C(i, j)=0 \\
0, C(i, j)=1
\end{array}\right.
$$

with $\mathrm{C}$ denoting the contiguity matrix. The first cost function represents the asymmetry principle and the second cost function also incorporates the contiguity principle and allows connections to originate from low and terminate to high hierarchical levels, provided that they involve contiguous regions. Thus, in order to code if two regions are contiguous or not, a contiguity matrix $\mathrm{C}$ was compiled with 0 and 1 as entries, where entry $\mathrm{C}(\mathrm{i}, \mathrm{j})=1$ denotes that regions $\mathrm{i}$ and $\mathrm{j}$ are contiguous. To this end Walker's map was consulted and entries formulated accordingly.

The minimization of En is achieved by the simulated annealing procedure. The initial value for $\mathrm{T}$ was set to the average degree of the network, i.e. $\mathrm{E} / \mathrm{N}$, as suggested in (Ispolatov \& Maslov, 2008). 1) After the initial random assignment of the nodes to $M$ hierarchy levels, En is computed. 2) A new assignment takes place and is accepted with a probability of $\min \left(1, \exp ^{\left(-\frac{\Delta E n}{T}\right)}\right)$ where $\Delta E n$ is the difference in the energy and $\mathrm{T}$ is the temperature. 3) After several reassignments at each temperature level $\mathrm{T}$, here set to $1000, \mathrm{~T}$ is decreased to $\mathrm{T}^{\prime}$ based on $\mathrm{T}^{\prime}=\mathrm{c}^{*} \mathrm{~T}$, where $\mathrm{c}$ is the cooling factor (here set to $0.9)$. 4) The procedure ends when $T<10^{-3}$ and thus the system has "cooled down". The resulting assignment offers an arrangement of the nodes over the $\mathrm{M}$ hierarchy levels. Hence, the dominant direction of the network originates from nodes placed in high hierarchical levels towards nodes placed in low hierarchical levels. Due to the stochastic nature of the approach, the procedure is repeated many times (here 1000) and the resulting arrangements/solutions can be used for the estimation of the frequency of assignment of each node to a particular hierarchical level.

The temperature $\mathrm{T}$ is set high enough in order to allow the exploration of all the states of the system and avoid getting stuck in local minima. The gradual cooling leads to less probable acceptance of configurations that do not minimize En and forces the system to 
move towards a global optimum. Moreover, the number of hierarchical levels $\mathrm{M}$ has to be defined empirically if no a priori information exists. This entails the application of the algorithm several times by varying the number of hierarchical levels. Subsequently the optimal number of levels is selected as the one that from which any further increase in the number of levels does not lead to a decrease in the cost/energy (Ispolatov and Maslov, 2008).

The ABH optimization arranges the nodes in levels but no direct information is provided for the hierarchical and anti-hierarchical connections of the network. In order to uncover such connections the following procedure was followed. For each of the 1000 solutions the hierarchical, from high to low levels, and anti-hierarchical, from low to high or same levels, connections were uncovered. Hence, this procedure produces a connectivity diagram quantifying the frequency that each connection is hierarchical or anti-hierarchical. In this way, we can assess the frequency with which each connection constitutes the dominant direction in the anatomical network (hierarchical connectivity diagram) and the frequency with which it goes against this dominant direction (antihierarchical connectivity diagram).

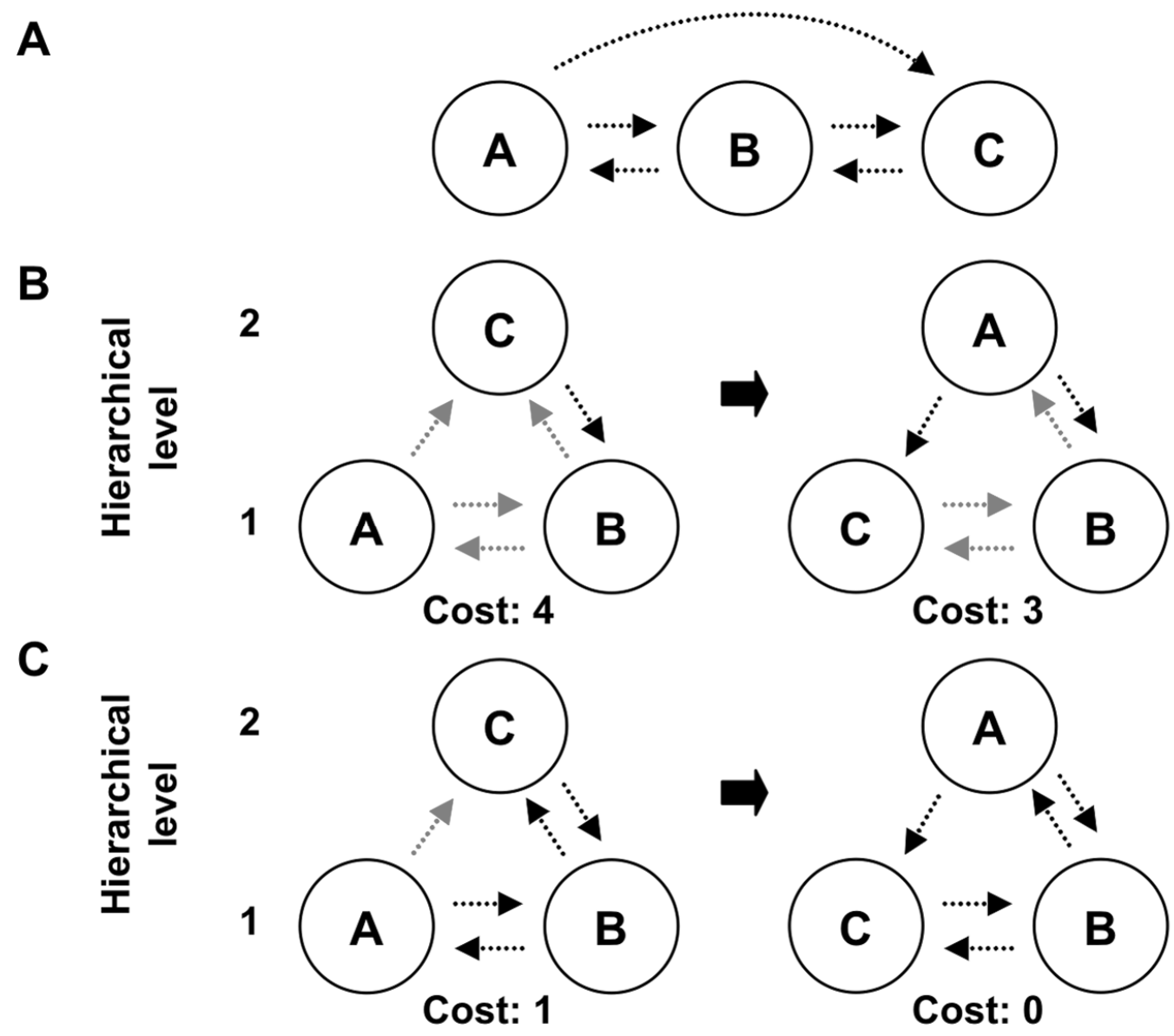

Figure 2. Example of the ABH optimization in a toy network. Given a directed network depicted in panel A the nodes are randomly assigned to $\mathrm{M}$ levels. Here for the sake of simplicity $\mathrm{M}=2$. B. Cost function 1 is used to compute the cost, i.e. number of anti-hierarchical arcs (coloured in light grey), at state 1 (left) leading to $\cos t=4$. After shuffling the nodes randomly (state 2 on the right) the cost is recomputed, i.e. cost $=3$. Hence, this state is favoured since it possesses a lower cost and corresponds to a "more hierarchical" arrangement of the nodes. C. The same example as in panel B but cost function 2 is employed, i.e. only anti-hierarchical arcs between non-contiguous regions are taken into account. This toy network is based on the illustration of the 


\section{Chapter 3}

principles of the anterior-posterior hierarchical model (see Fig. 4 in Badre and D'Esposito, 2009). For the sake of simplicity, only 2 hierarchical levels are used.

Network metrics

Finally network metrics (Kötter and Stephan, 2003; Rubinov and Sporns, 2010) will be employed in order to examine how each region is embedded within the PFC network. This will allow us to examine how and if network properties are related to the hierarchical position of each region. Two basic network properties will be examined, i.e. centrality and segregation. The centrality of each region will be assessed with the betweenness centrality $(\mathrm{BC})$. Higher $\mathrm{BC}$ values for a node indicate that the node participates in a large fraction of shortest paths between the nodes of the network. Hence, such nodes can be viewed as an "intermediate" station of network traffic. The segregation of the regions will be quantified with the $\mathrm{CC}$ (CC). The $\mathrm{CC}$ of a node quantifies the probability of the existence of direct connections between its neighbouring, i.e. directly connected, nodes. Hence, higher values indicate "cooperative" relations between the neighbours of a node and are thought to reflect higher levels of segregation (Rubinov and Sporns, 2010). Moreover, we will use the transmission index that captures the efferents to afferents relation (Kötter and Stephan, 2003). Higher values indicate that a region exhibits more efferent than afferent connections. We use the transmission index since it is closely linked to the current concept of $\mathrm{ABH}$. Regions with higher transmission index values are more suited for higher levels within the hierarchy. All the above measures were computed by using the formulas for directed networks described in (Kötter and Stephan, 2003; Rubinov and Sporns, 2010).

The significance of the $\mathrm{CC}$ and the $\mathrm{BC}$ will be assessed by comparisons with the values obtained from 1000 random networks matched in the number of connections, nodes and degree, i.e. number of connections, of nodes (e.g. Honey et al., 2007) resulting in a $\mathrm{z}$-score and $\mathrm{p}$ value for the $\mathrm{CC}$ and the $\mathrm{BC}$ for each region.

The network metrics of each region will be correlated with their $\mathrm{ABH}$ level in order to assess potential relations. For instance, regions higher in the hierarchy, and thus functioning as regulators, might possess lower CC and thus appear less segregated, influencing a diffuse set of regions, while regions successively lower in the hierarchy might exhibit an increased level of segregation. We expect that the transmission index will exhibit high correlation with the hierarchy level, since higher values dictate a "surplus" of efferents, and such regions should end up in high hierarchical levels during the $\mathrm{ABH}$ optimization procedure.

\section{Reference "maximally" and "minimally" ABH networks}

The previous analysis aims at answering the following questions: How are the PFC regions distributed in the hierarchical levels produced by the $\mathrm{ABH}$ optimization procedure? How do these results relate to the predictions of the hierarchical anteriorposterior model (Badre and D'Esposito, 2009)?

We next sought to answer a different question: Does the PFC structural network possess a "natural" topology that corresponds to the hierarchy principles of the hierarchical anterior-posterior model? Hence, we examined if the PFC structural network corresponds to a topology with the minimal possible amount of antihierarchical connections, and in that sense, if it differs from a "maximally" ABH 
network. Moreover, we wanted to examine if the macaque PFC topology differs from the other "extreme", i.e. a "minimally" ABH network exhibiting a maximal amount of anti-hierarchical connections. To this end, we constructed ad hoc reference "maximally" and "minimally" ABH networks by rewiring the connections of the original network.

The maximally and minimally $\mathrm{ABH}$ networks were constructed by an annealing procedure. Initially, a "seed" network was generated by rewiring the edges of the original network and preserving the node degree. Subsequently the number of antihierarchical connections was calculated as the cost of the "seed" network. By using this "seed" network a simulated annealing process was used but in this case the energy to be minimized is defined as:

$$
E n=\left(\text { Cost }_{\text {Desired }}-\text { Cost }_{\text {Actual }}\right)^{2}
$$

Hence, for the generation of the maximally (minimally) $\mathrm{ABH}$ networks we set as the desired cost in eq. 3 a number much lower (higher) than the cost obtained in the hierarchy optimization of the original network. At each step of the annealing procedure a new rewired network is generated, with a wiring probability of $\min \left(1, \exp ^{\left(-\frac{\Delta E n}{T}\right)}\right)$ and accepted with probability of $\min \left(1, \exp ^{\left(-\frac{\Delta E n}{T}\right)}\right)$. We should note that the annealing procedure previously described, i.e. the $\mathrm{ABH}$ optimization that minimizes eq. 1 and 2, is "embedded" within the annealing procedure minimizing eq. 3 , in order to calculate the cost associated with each step. In this way, we search for networks with the exact same number of nodes, edges and node degree as the original one but with a maximum or minimum number of anti-hierarchical connections. The whole procedure was repeated 100 times and thus 100 maximally and minimally $\mathrm{ABH}$ reference networks were generated. Similar approaches have been used in order to construct networks that optimize certain features such as transitivity (Maslov et al., 2007). Moreover, the underlying rationale of this approach resembles strategies used to assess the underlying wiring principles of the macaque cortex, i.e. if it is wired in such a way to minimize wiring length (Kaiser and Hilgetag, 2006).

By comparing the amount of anti-hierarchical connections of the original network with the ones of the maximally and minimally $\mathrm{ABH}$ reference networks we are able to examine how the original network is related to these two "extremes", i.e. if it exhibits a significantly different amount of anti-hierarchical connections.

Laminar based hierarchy optimization

As a final analysis step, we performed a LBH optimization based on a different concept of hierarchy based on laminar patterns of connections (Barbas and RempelClower, 1997).

It has been shown that the PFC regions can be differentiated with respect to their degree of eulamination (Barbas and Rempel-Clower, 1997; Dombrowski et al., 2001). The PFC regions can be ranked in 5 levels of structural type with 1 denoting the less eulaminated ones, e.g. region 13, and 5 denoting the more eulaminated, e.g. region 45. With this classification a connection from region $\mathrm{i}$ to region $\mathrm{j}$ can be associated with an index Delta defined as: Delta $=$ Structural type origin - Structural type destination .Hence, negative (positive) Delta values denote connections from less (more) eulaminated to 


\section{Chapter 3}

more (less) eulaminated regions. This structural model is an excellent predictor of the laminar patterns of connections between the PFC regions (Barbas and Rempel-Clower, 1997). More specifically, an almost perfect positive correlation was observed between the Delta index associated with a connection from region $\mathrm{i}$ to region $\mathrm{j}$ and the percentage of anterograde labelling in layers IV-VI (\% AL IV-VI). Consequently, the structural type of two connected regions is an excellent predictor of the observed \% AL IV-VI with a higher (lower) \% AL IV-VI denoting an increasingly FF (FB) connection. Hence, connections originating from less (more) eulaminated regions and targeting more (less) eulaminated ones are comparable to FB (FF) connections. Thus, this information can be used for a $\mathrm{LBH}$ arrangement of the PFC regions. We should note that the FF and FB connections among PFC regions bear a resemblance with FF and FB connections in the sensory cortices. However, while FF connections terminate in layer IV in sensory cortices, the PFC FF connections appear more diffuse by terminating in layers IV-VI (Barbas and Rempel-Clower, 1997).

For the LBH the following procedure was adopted. We based our analysis on the findings reported in (Barbas and Rempel-Clower, 1997). First, since no direct information for the \% AL IV-VI of each PFC connection was available, we relied on a summary based on the corresponding Delta index of the PFC connections (Barbas and Rempel-Clower, 1997). We assigned the various regions of Walker's parcellation schemes to a structural type level. As an intermediate step, an approximate correspondance was established between Walker's parcellation scheme and the PFC parcellation scheme used in Barbas and Rempel-Clower, 1997 by using the mapping relations from the CoCoMac database. Second, we used the classification scheme of 5 levels of structural type to assign a level to each region of Walker's parcellation (Supplementary Table 4). Third, for each connection between the PFC regions a Delta index was computed as described above and assigned to the connection. The Delta index was used to assign a \% AL IV-VI value to each connection by using the values reported in (Barbas and Rempel-Clower, 1997). For Delta values that were not available in the aforementioned study, the \% AL IV-VI values were "quasi-interpolated". For instance for Delta values of 2.5 we assigned a \% AL IV-VI value below the one corresponding to Delta ranges of 3 and above the ones corresponding to 2. Finally, in order to use the \% AL IV-VI values to denote FF and FB connections, a "Laminar Bias" (LB) index was calculated as LB=50 - \% AL IV-VI (Supplementary Fig. 1). Hence, with the current definitions, the LB index ranges from -50 to +50 , with values towards 50 denoting increasingly $\mathrm{FF}$ connections and values toward +50 increasingly $\mathrm{FB}$ connections. The goal of a LBH arrangement is to produce a layout of the PFC regions with as many as possible FF connections pointing upwards and FB connections point downwards. In more formal terms we seek to minimize the cost/energy defined as:

$$
E n=\sum_{i \rightarrow j}\left[g\left(\left(m_{i}-m_{j}\right) \cdot L B_{i \rightarrow j}\right) \cdot \operatorname{abs}\left(L B_{i \rightarrow j}\right)\right]
$$

where:

$$
g(x)= \begin{cases}0, & x>0 \\ 1, & x \leq 0\end{cases}
$$


where $i \rightarrow j$ denotes a structural connection from region $\mathrm{i}$ to region $\mathrm{j}, L B_{i \rightarrow j}$ denotes the laminar bias associated with this connection, and $\mathrm{m}_{\mathrm{i}}$ and $\mathrm{m}_{\mathrm{j}}$ is the hierarchical level of node $\mathrm{i}$ and $\mathrm{j}$. The minimization of the energy and the optimal choice of levels were performed as described in the ABH optimization. A schematic depiction of the approach is illustrated in Fig. 3. Similar approaches have been adopted for the laminar based hierarchical arrangement of the cat visual cortex (Hilgetag and Grant, 2010). We should note that we based the above procedure on the connectivity of PFC regions as indicated by the dataset Walker 1 .

In order to relate the results from the $\mathrm{ABH}$ and $\mathrm{LBH}$ optimization, the correlation between the hierarchical levels for the two optimization procedures will be assessed. Moreover, for each efferent termed as hierarchical or anti-hierarchical during the $\mathrm{ABH}$ optimization, a corresponding LB index will be calculated as the mean of the values of the positive and negative LB values for each efferent. In this way, information on the laminar patterns of hierarchical or anti-hierarchical PFC connections according to the $\mathrm{ABH}$, and thus how they correspond to the $\mathrm{FF}$ and $\mathrm{FB}$ classifications scheme, is readily available and offer a richer picture of the PFC connectivity.

All analyses were performed with functions from the Brain Connectivity Toolbox (Rubinov and Sporns, 2010), custom software written in Matlab and modified freely available Matlab functions (http://www.cmth.bnl.gov/ maslov/programs.htm). All datasets and scripts used are available upon request.

\section{$\underline{\text { Results }}$}

\section{ABH optimization of the PFC regions}

The ABH optimization with Walker 1 and cost function (1) resulted in solutions with 8 hierarchical levels and equal cost (Table 2, Fig. 4 A, 5 A). Regions 25 and 45, medial and lateral respectively, were assigned at the top of the hierarchy with a very stable assignment across the estimated solutions, i.e. in all solutions these regions were placed at the top level (Fig. 5 A). These were followed by lateral region 46 and orbital region 13 and 12 with a peak of the frequency of assignment at level 7. All the other regions had unique peaks and narrow distributions over the solutions, with the exception of region 14 (Fig. 5 A). Interestingly, region 10 was placed at lower levels with a peak at level 4, much lower than the more posterior regions 46 and 45. Moreover, lateral region $8 \mathrm{~A}$ was placed towards the bottom of the hierarchy with a peak at level 2 . Hence, the hierarchical arrangement does not follow an anterior-posterior gradient with anterior regions placed higher in the hierarchy than posterior ones. A rather similar picture, with the exception that region 8A was assigned to higher levels, emerged when Walker 1 and cost function (2) was used for the ABH optimization. This combination led to solutions with equal cost and 4 hierarchical levels (Table 2, Fig. 4 B, 5 B). Very similar results were obtained when the hierarchy optimization with cost function (1) was applied to the Walker 2 dataset resulting in 8 hierarchical levels (Table 2, Fig. 4 C, 5 C). Despite certain variations in the resulting hierarchical lay out, lateral regions 45 and 46 dominated again the hierarchy and were placed with high consistency at the higher level, while the more anterior region 10 was placed at lower levels (Fig. 5 C). Even when using a different connectivity matrix, i.e. Modha and Singh PFC (Supplementary Table 1), for the $\mathrm{ABH}$ optimization, lateral region 45 was consistently placed at the higher levels of the hierarchy and never placed below the more anterior region 10 . The 
Modha and Singh PFC dataset is more fine grained since it contains multiple subdivisions of the gross Walker parcellation scheme. Hence, we can gain insights about lateral, medial and orbital parts of e.g. region 10 and their position in the $A B H$ arrangement. The only subdivision of region 10 that was placed high in the hierarchy was subdivision 10m (Carmichael and Price, 1994). Even in that case it was placed consistently lower than subdivisions of more posterior region 45, with the rest of its subdivisions placed lower than the (sub)divisions of the more posterior region 46 (Supplementary Table 1). Taken together the above suggest that a strict anteriorposterior gradient does not hold. Regions 45 and 46 were consistently placed at the top levels of the hierarchy, surpassing the most anterior region 10. Only regions 46 and $8 \mathrm{~A}$ seems to have a hierarchical relation in line with the predictions of the hierarchical anterior-posterior model (Badre and D'Esposito, 2009). The above held true irrespective of cost function, connectivity dataset and parcellation scheme.

A
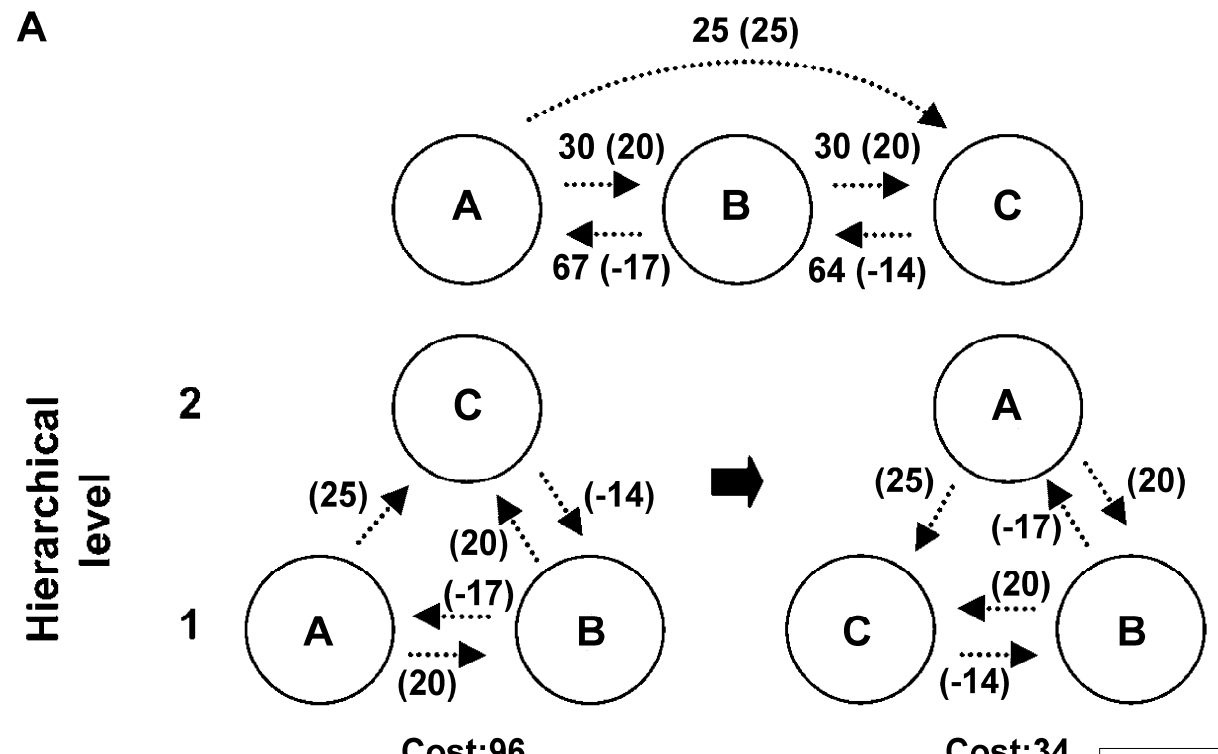

Cost:96

Cost:34

B

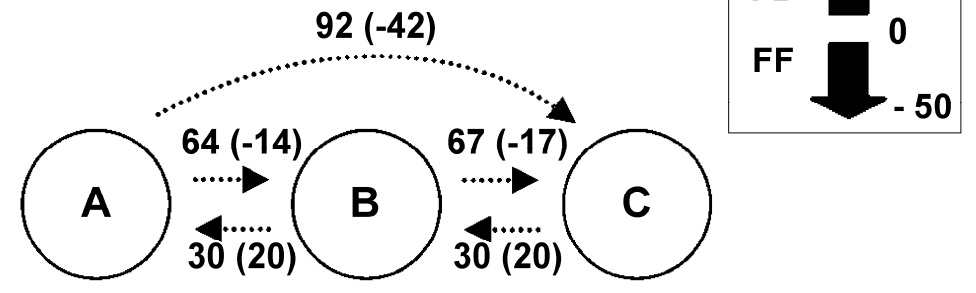

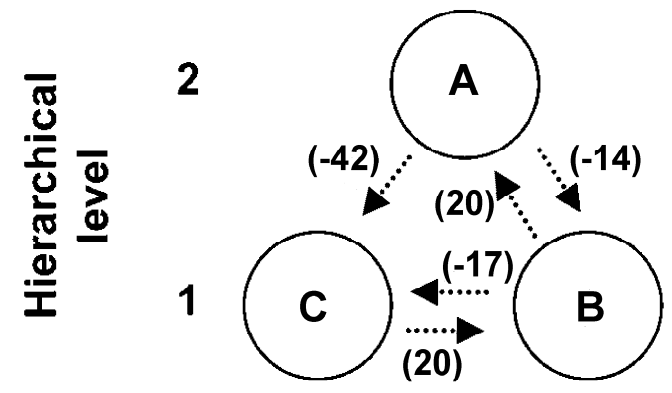

Cost:113

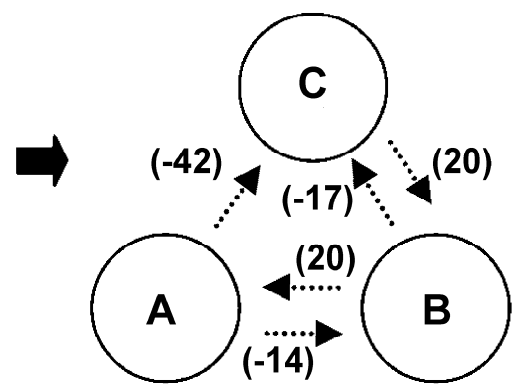

Cost:34 
Figure 3. Example of the LBH optimization in a toy network. The toy network involves the same set of regions (A, B and C) and connections as in Fig. 2. Since the LBH relies on the laminar patterns of connections, a \% AL IV-VI value must be assigned to the connections. We used the structural model as described in (Barbas and Rempel-Clower, 1997) to assign such a value based on the level of differentiation of each region. Numbers above each connection represent \% AL IV-VI values and numbers in parenthesis the corresponding LB indexes. A. In this scenario regions $\mathrm{A}, \mathrm{B}$ and $\mathrm{C}$ belong to the structural types 2,3 , and 5 respectively. Hence, $\mathrm{A}$ is the least and $\mathrm{C}$ the most differentiated region. $\mathrm{B}$. In this scenario regions $\mathrm{A}, \mathrm{B}$ and $\mathrm{C}$ belong to the structural types 5, 3, and 2 respectively. In both scenarios the LBH optimization seeks to arrange the regions in levels in such a way that connections with negative LB index (FF) point upwards and connections with positive LB index (FB) point downwards. Hence, the "optimal" arrangement corresponds to the ones depicted on the right side of each panel, since they exhibit a lower cost that the ones depicted on the left side of each panel. Note that this toy network is the same as in Fig 2 involving the exact same set of regions and connections, but according to the structural type of each region, illustrated in the above scenarios, the LBH might coincide (panel A scenario 1) or differ (panel B scenario 2) with the $\mathrm{ABH}$ layout.

By taking into account the PFC as a whole we could assess the hierarchical role of orbital and medial regions. Orbital region 13 was consistently assigned to high levels in the hierarchy, dominating most of the other orbital regions. This was observed when using dataset Walker 1 and cost function (1) (Fig. 5 A), cost function (2) (Fig. 5 B), dataset Walker 2 (Fig 5 C) and the Modha and Singh PFC dataset (Supplementary Table 1). In the later analyses region 13 was only surpassed by the orbital regions 12, OFap (orbital periallocortex, Morecraft et al., 1992) and Gu (gustatory cortex, Suzuki and Amaral, 1994) which are located at approximately the same or lower level along the anterior-posterior axis. With respect to the medial part of the PFC, region 25 was always placed in higher levels than the more posterior region 24 (Fig. 5). When using the Modha and Singh PFC dataset, region 32, corresponding to region 25 in Walker map (Kötter et al., 2001), was also consistently placed at high levels of the hierarchy (Supplementary Table 1). Hence, with respect to the orbital PFC the more posterior regions occupy high levels of the hierarchy. Regions in the medial wall follow another pattern, i.e. region 25 is placed in higher levels than the more posterior region 24.

In order to investigate if the choice of a particular set of regions changes the results we selected only the lateral PFC regions, the "cognitive" part of the PFC emphasized in (Badre and D'Esposito, 2009), and performed the ABH optimization. No anteriorposterior gradient was observed and region 10 was not assigned to the higher levels (Supplementary Tables 2, 3). Hence, the choice of only the lateral PFC regions does not alter the results and conclusions.

In sum, the $\mathrm{ABH}$ optimization does not confirm an anterior-posterior hierarchical gradient with region 10 on top of the hierarchy. The choice of connectivity dataset and cost functions does not significantly alter the results (Table 3). Moreover, out of the 1000 solutions there were different solutions with a high degree of dissimilarity and the exact same cost (Table 2). In other words, no unique arrangement best describes the $\mathrm{ABH}$ ordering of the PFC, a phenomenon analogous to the indeterminacy of the visual system (Hilgetag et al., 1996). 
Table 2. Solutions obtained for the ABH optimization.

\begin{tabular}{|l|c|c|c|c|}
\hline & $\begin{array}{c}\text { Nr of } \\
\text { unique } \\
\text { solutions }\end{array}$ & $\begin{array}{c}\text { Similarity of unique solutions } \\
\text { (Hamming distance) }\end{array}$ & $\begin{array}{c}\text { Cost (\% of } \\
\text { edges deleted) }\end{array}$ \\
\hline & & Mean & Std & \\
\hline Walker 1 & 251 & 0.594 & 0.164 & 0,422 \\
\hline Walker 1 cost 2 & 19 & 0.426 & 0.194 & 0,383 \\
\hline Walker 2 & 474 & 0.598 & 0.153 & 0,442 \\
\hline
\end{tabular}

Characteristics of the 1000 solutions obtained from the hierarchy optimization. Note that all solutions correspond to the exact same cost. Out of the 1000 solutions there were unique solutions exhibiting a high degree of dissimilarity, as indicated by the Hamming distance, and equal cost. The cost is expressed as the number of anti-hierarchical connections/edges over all the existing PFC connections/edges.

Hierarchical and anti-hierarchical diagrams for $\mathrm{ABH}$ optimization

The hierarchical diagram demonstrates that the regions at the top of the hierarchy, i.e. $25,45,46,13,12$, exhibit diffuse and extensive projections with all the PFC groups as specified in (Kötter et al., 2007), i.e. orbitomedial, lateral and intermediate (Fig. 6). We will use this PFC grouping nomenclature throughout the text. The only exception is region 13 that does not send efferents to the lateral group. The efferents of these regions were termed as hierarchical with very high frequency over the 1000 solutions. Hence, they constitute the dominant direction towards regions at lower hierarchical levels. Regions at the lower parts of the hierarchy are 9, 24 and 11 and they constitute the "end points" of the dominant direction in the PFC anatomical network (Fig. 6). The latter regions also exhibit massive anti-hierarchical efferents that influence the regions of all the PFC groups, i.e. orbitomedial, lateral and intermediate (Fig. 7). The only exception is the less diffuse pattern of efferents of region 11 that does not exhibit anti-hierarchical efferents with the lateral group. The efferents of the regions low at the hierarchy were termed as anti-hierarchical with a high frequency over the 1000 solutions and thus constitute the main anatomical routes through which information can be sent back to regions that dominate the hierarchy. Both the hierarchical and anti-hierarchical efferents traverse several levels and can thus directly link regions at the opposite sides of the hierarchy, i.e. high with low regions and vice versa, without the necessity of intermediate regions. Thus, the structural architecture does not imply a cascade-like way of information flow (see also Discussion). 
Table 3. Similarity of ABH scores across datasets and cost functions.

\begin{tabular}{|c|c|c|c|}
\hline & Walker1 & Walker1 cost 2 & Walker 2 \\
\hline Walker 1 & NA & 0.66 & 0.95 \\
\hline Walker 1 cost 2 & $\mathrm{p}<0.05$ & NA & 0.60 \\
\hline Walker 2 & $\mathrm{p}<0.001$ & $\mathrm{p}<0.05$ & NA \\
\hline
\end{tabular}

Quantification of the similarity of the results of the hierarchy optimization with different datasets and cost functions. The hierarchy score from each combination was calculated as the mean hierarchical levels attributed to a region across the 1000 solutions. Subsequently all pairwise correlations between the hierarchical scores were computed and the significance of the observed correlation was assessed with randomization statistics.

Network metrics and hierarchy

The network metrics revealed that the only regions with significant $\mathrm{BC}$ (BC $\mathrm{z}$-score $>$ $2, \mathrm{p}<0.001$ ), and consequently highly central in the PFC network, belong to the intermediate PFC group, i.e. regions 9 and 24 (Fig. 8 A). This finding is in line with analysis of the PFC using Shapley ratings which revealed regions 9 and 24 as among the ones with the higher contribution to global PFC connectivity (Kötter et al., 2007). The $\mathrm{CC}$ involved only regions of the orbitomedial group, i.e. 11 and 14 (C z-score $>2$, $\mathrm{p}<0.001$ ) (Fig. 8 B). Thus, these regions appear to be more segregated/specialized. The transmission index has been computed before for all the regions for the current dataset (Kötter and Stephan, 2003) and hence the current results are identical: the lateral group exhibits the highest transmission values above 0.5 (regions 45 and 46), exhibiting a "surplus of efferents", while the intermediate group exhibited values below 0.5, indicating a predominance of afferents. The orbitomedial group exhibited transmission values around 0.5 (Fig. $8 \mathrm{C}$ ). In order to directly examine the relation of the networks metrics and the $\mathrm{ABH}$ level of the region, the hierarchy score, i.e. the mean hierarchical level across 1000 solutions that a region was assigned to, of each region was correlated with each network metric. The only metric that exhibited a significant correlation was the transmission index $(\mathrm{r}=0.72 \mathrm{p}<0.05)$. This is expected since the transmission index quantifies the efferents to afferents relation, with values higher than 0.5 denoting a surplus of efferents. Hence, it is logical that the regions with high (or low) transmission index end up in high (or low) hierarchical levels during the ABH optimization.

We should also note that no significant correlation was observed for the LBH and any of the network metrics.

\section{Relation to "maximally" and "minimally" ABH networks}

The costs obtained from the maximally $\mathrm{ABH}$ networks resulted in a modest but significant reduction in the cost compared to the original networks (Fig. 9). These results indicate that the original network topology has an "overabundance" of antihierarchical connections since the reference maximally $\mathrm{ABH}$ networks exhibited significantly less anti-hierarchical connections. The costs obtained from the minimally $\mathrm{ABH}$ networks were on average slightly higher than the cost of the original network and did not reach significance (Fig. 9). The above results held true for all datasets, i.e. Walker, Walker 2 as well as for both cost functions (Fig. 9). Taken together the above 
results dictate that the topology of the PFC is wired in such a way that does not comply to an "optimal" maximally ABH topology. Instead it lies close to networks exhibiting an "overabundance" of anti-hierarchical connections and thus the PFC wiring could be considered as minimally hierarchical. Importantly, the ABH arrangement of the regions of the "maximally" and "minimally" ABH networks was highly similar as the one of the original network. Hence, no hierarchical anterior-posterior gradient, with region 10 on top, was observed for these networks either.

\section{Laminar based hierarchy}

The LBH optimization resulted in a layout with 6 levels with, as expected given the way the LB index was estimated, the more (less) differentiated regions occupying low (high) levels of the hierarchy (Supplementary Table 5).

In order to assess how the $\mathrm{ABH}$ and $\mathrm{LBH}$ optimization results relate, we computed the correlation between the mean hierarchical level for each region over the 1000 solutions for the $\mathrm{ABH}$ and the $\mathrm{LBH}$. The results from the two hierarchy optimization procedures exhibited a near 0 correlation that was not significant $(\mathrm{P}>0.1)$.

For a visual depiction of the results and in order to gain more information from the results of the two hierarchy optimizations, we plotted the $\mathrm{ABH}$ against the $\mathrm{LBH}$ mean level for every PFC region. Moreover, a plane was drawn at the mean of the ABH and LBH mean levels dividing the scatterplot in 4 parts (Fig. 10). This dissection can be used as a "classification scheme" for the PFC regions. For instance, regions like 25 and 45 are located at high $\mathrm{ABH}$ levels and thus function as broadcasters. The LBH optimization further differentiates these broadcasting structures: while region 25 is also located at high levels according to the LBH, region 45 is located at very low ones (Fig 10). Thus, the hierarchical efferents, according to the $\mathrm{ABH}$, that region 25 exhibits constitute FB connections, whereas the hierarchical efferents of region 45 constitute FF connections (Fig. 11 A). FB and FF connections target different laminae and thus influence a different microenvironment containing distinct types of neurons (Dombrowski et al., 2001; Barbas and Hilgetag, 2002) resulting in a complex dialogue between the PFC regions. The $\mathrm{ABH}$ in conjunction with the $\mathrm{LBH}$ can summarize and pinpoint aspects of this complex dialogue (see Discussion). 

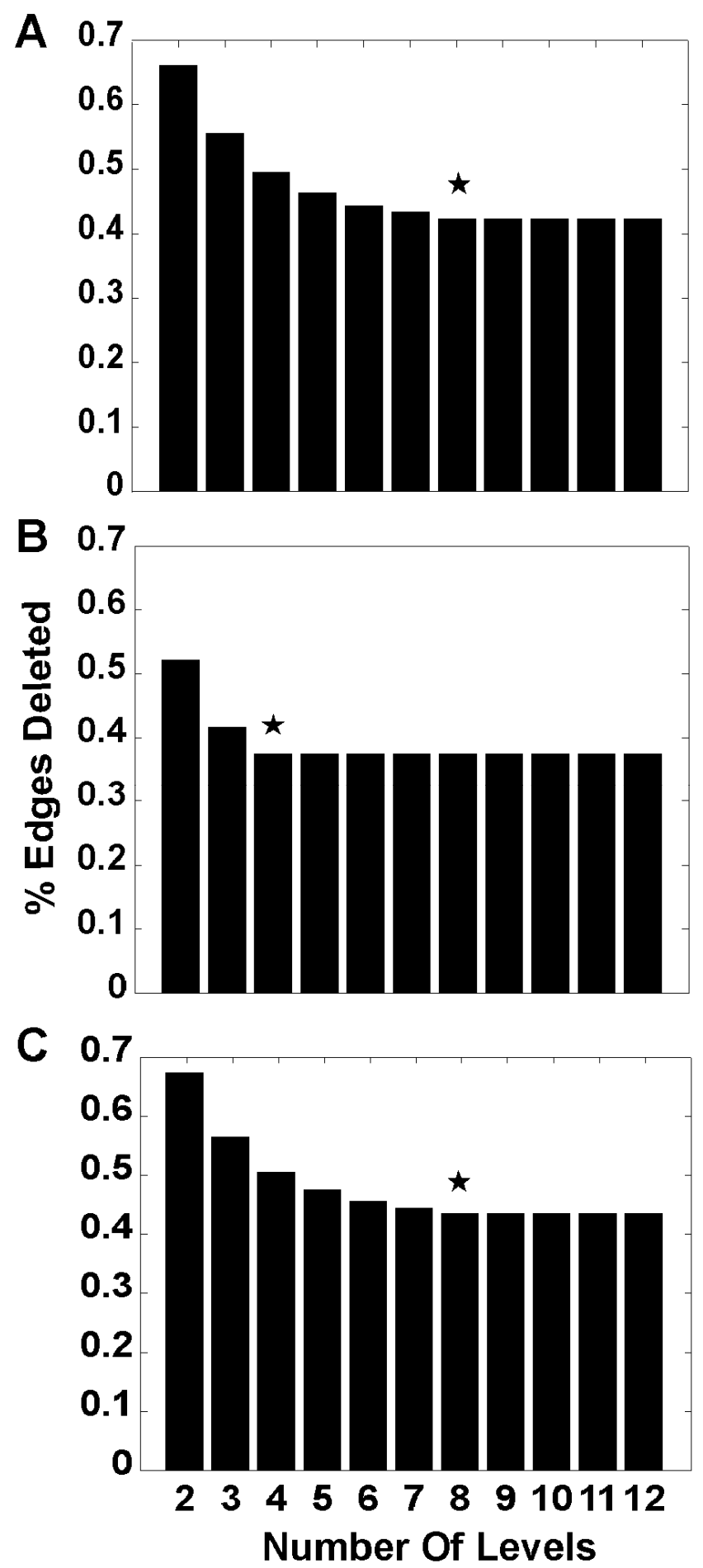

Figure 4. Empirical establishment of the optimal number of levels for the ABH optimization. Walker 1 and A. Cost function $1 \mathrm{~B}$. Cost function $2 \mathrm{C}$. Walker 2 and Cost function 1 . The stars point to the levels from which any further increase of the number of levels does not result in a reduction of the cost. These levels were used for the $\mathrm{ABH}$ optimization. The cost is expressed as a percentage of the edges deleted over the total number of edges contained in the graph. 


\section{Chapter 3}

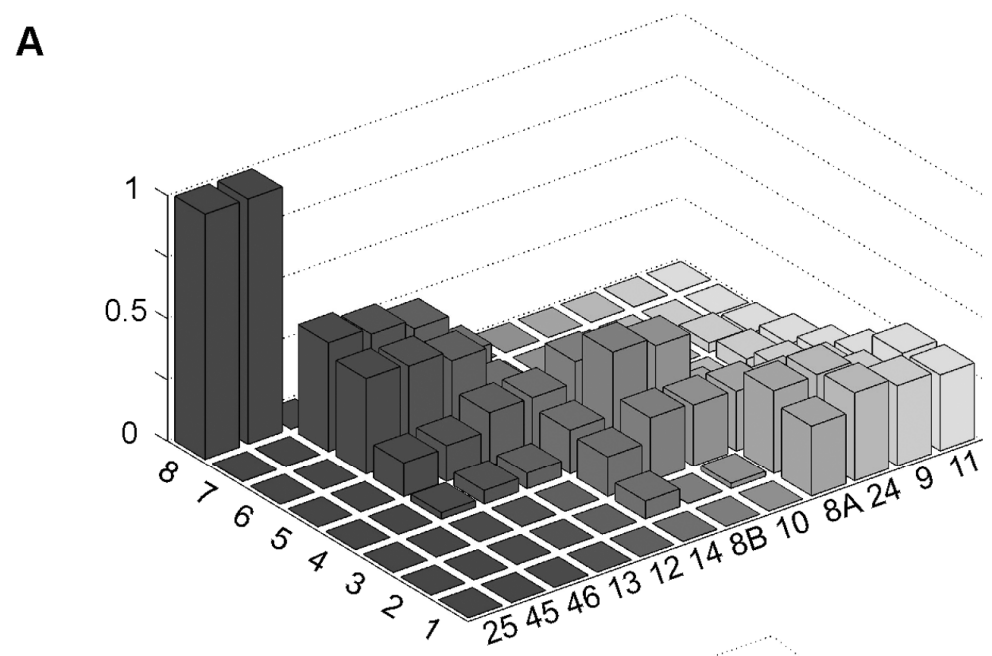

B
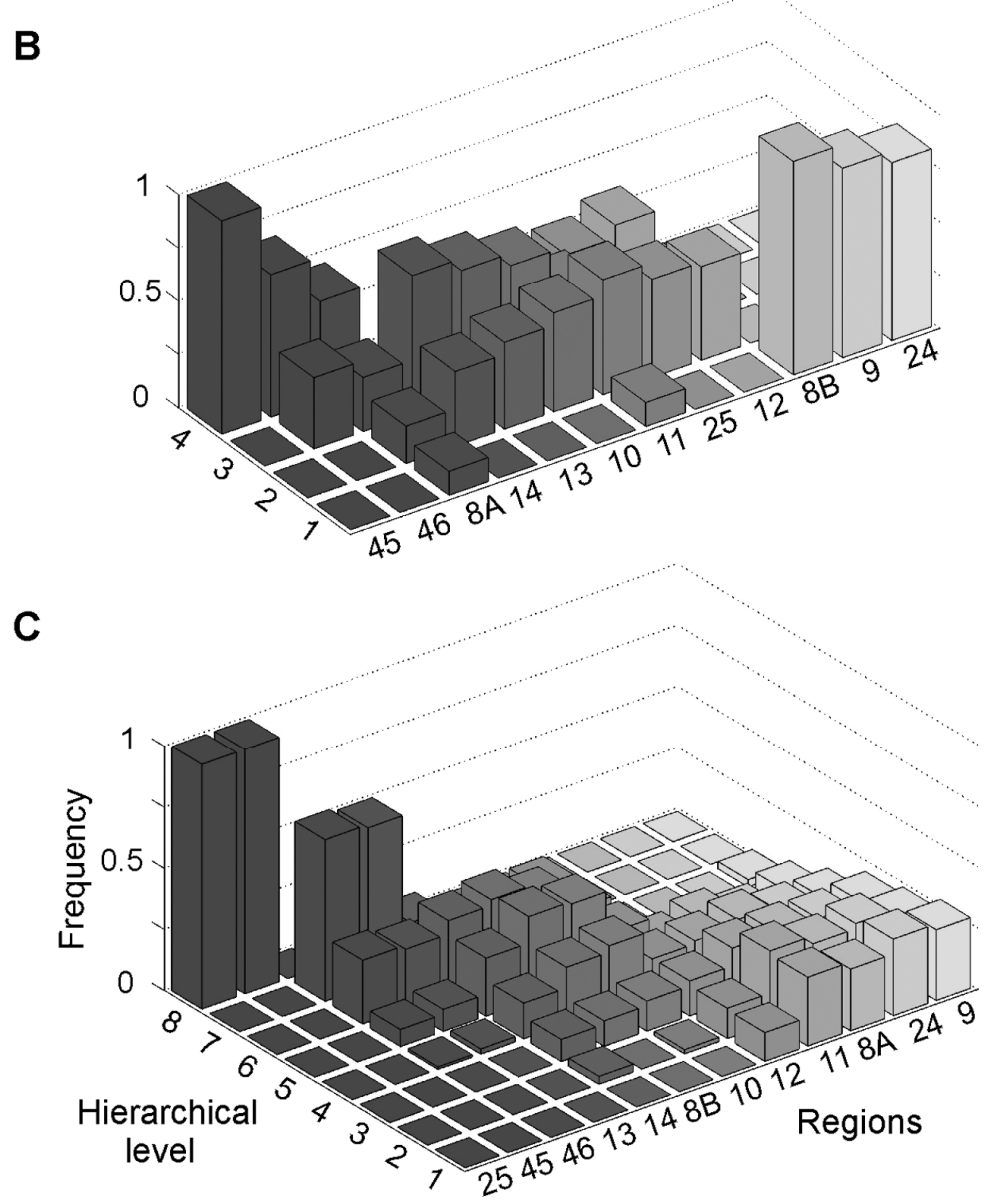

Figure 5. Distribution of solutions of the ABH optimization. Dataset Walker 1 and A. Cost function 1 B. Cost function 2 C. Dataset Walker 2 and Cost function 1. Figures depict the frequency of assignment of the PFC regions at each hierarchical level over the 1000 solutions. The regions are sorted according to the level where they exhibited the highest frequency peak. Regions with the frequency peak involving the same level are further sorted according to their frequency. Darker (lighter) shades of grey denote regions that occupy high (low) levels of the hierarchy. Note that the distribution of the solutions appears segregated with distinct picks. Such distribution differs from the "flat" distribution of solutions that would be obtained from networks lacking a prominent dominant direction, e.g. networks with symmetrical topology (all connections are bidirectional). 


\section{$\underline{\text { Discussion }}$}

In the current study we took a formal stance and used a wealth of data on the anatomical connections of the macaque PFC in order to quantify the principles and predictions of the hierarchical anterior-posterior model (Badre and D'Esposito, 2009). Below we discuss the main findings of our analyses.

\section{Relation to the hierarchical anterior-posterior model and contribution to PFC function}

The $\mathrm{ABH}$ results suggest that the dominant direction in the PFC stems primarily from regions $45,25,46$ and 13 , since they were consistently placed at the higher hierarchical levels. Region 45 has been identified as a broadcasting structure influencing the entire PFC (Kötter et al., 2001). Our analysis illustrates that regions lying on high hierarchical levels, i.e. regions 46, 25, 13, also function as "outflow hubs" or broadcasting structures (Kötter and Stephan, 2003) (Fig. 6). Contrary to the hierarchical anterior-posterior model region 10 did not lie on top of the hierarchy. This region was always surpassed by more posterior lateral PFC regions 45 and 46 (Fig. 5). Thus the most anterior region 10 and its lateral subdivisions (Supplementary Table 1) do not belong to the top of the hierarchy and more posterior lateral regions, i.e. 45, 46 (and anterior subdivisions of the later, i.e. 46v) appear more hierarchical (Fig. 5, Supplementary Table 1). Hence, the hierarchy optimization revealed a set of highly influential regions that are dispersed in the lateral, medial and orbital surfaces of the PFC. These broadcasting regions can have an analogous role as regulatory nodes in metabolic networks (Ispolatov and Maslov, 2008). Hence, with the asymmetry based concept of hierarchy and by taking into account the structural connectivity of the macaque PFC, the hierarchical arrangement of the PFC regions do not support an anterior-posterior hierarchical gradient with more anterior regions higher in the hierarchy.

The broadcasting structures are dispersed throughout the PFC surfaces, which have been differentially implicated in the cognitive, motivational and affective domain (Groenewegen and Uylings, 2000). Hence, the influence exerted by each of these structures can have a different contribution to PFC function. Regions in the posterior part of the orbital PFC, e.g. region 13, are multimodal and receive information from gustatory, visual, auditory, somatosensory and olfactory areas (Barbas, 2007). Thus, the dominant direction within the PFC that stems from posterior orbital PFC regions, placed high in the hierarchy, can propagate information from diverse modalities. Moreover, regions at the posterior orbital PFC are characterized by dense projections from the amygdala and might be responsible for the attribution of emotional valence to stimuli (Barbas, 2007; Ghashghaei et al., 2007; Murray, 2007). Consequently, the influence that region 13 exerts as a broadcasting structure might also subserve the propagation of motivational/reward signals through the rest of the PFC. Additionally, the hierarchical efferents, as revealed by the $\mathrm{ABH}$, have a positive $\mathrm{LB}$ index and thus constitute $\mathrm{FB}$ connections (Fig 11 A). The topology of Walker's region 45 and the so called Broca regions in the human brain (Petrides and Pandya, 1994) might suggest possible homologies. Consequently, its connectivity might constitute a neural circuitry that functions as a predecessor of language related circuitry in the human PFC (Kötter and Stephan, 2003). However, it should be noted that Walker's parcellation scheme was not performed on a comparative manner, and thus Walker's region 45 might not coincide with what is demarcated as region 45 in (Petrides and Pandya, 1994). With respect to the medial PFC, region 25 was consistently placed at high hierarchical levels (Fig. 5). 


\section{Chapter 3}

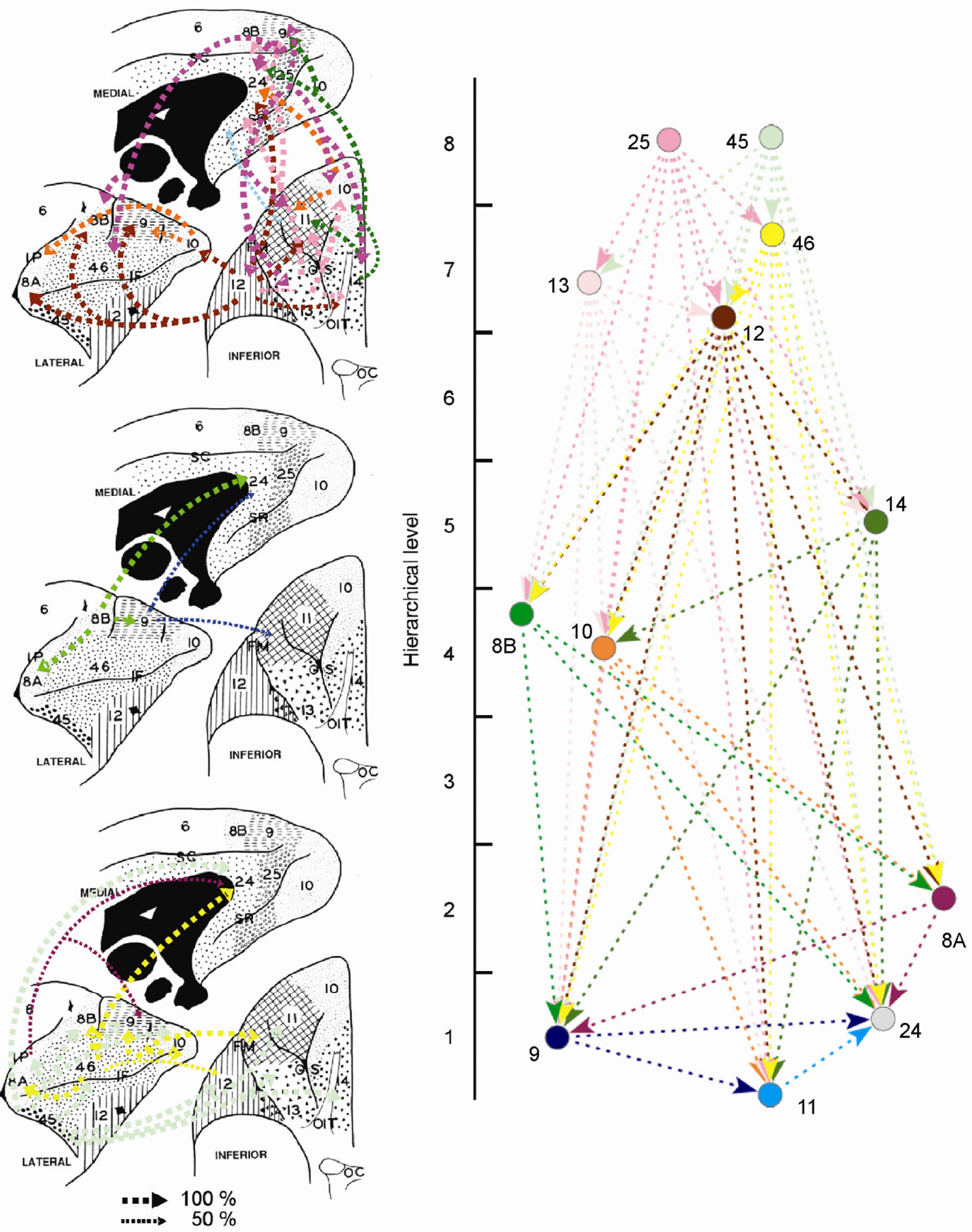

Figure 6. Hierarchical diagram based on 1000 solutions obtained from the ABH optimization for dataset Walker 1 and cost function 1. Each edge represents the frequency that an efferent was termed hierarchical with thicker lines representing the most frequent ones. Only efferents with a frequency higher than $50 \%$ were included. The representation overlaid on Walker's parcellation scheme offers an anatomical view of the regions involved in the more frequent hierarchical efferents and offers an overview of the ABH layout. The graph representation offers an overview of the distribution of regions over the ABH levels. 

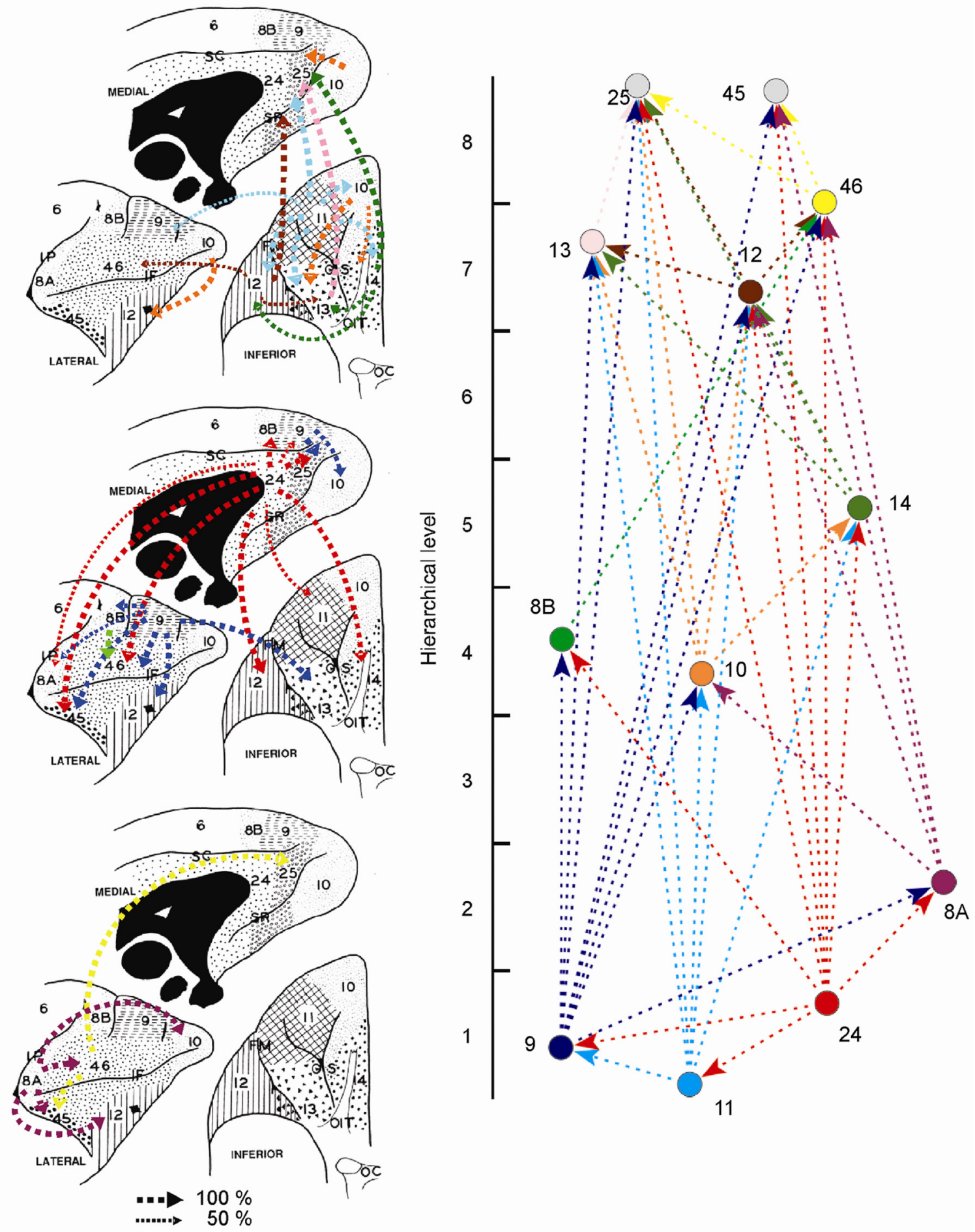

11

Figure 7. Anti-hierarchical diagram based on 1000 solutions obtained from the ABH optimization for dataset Walker 1 and cost function 1. Notation and arrangement of regions as described in Fig. 6. 

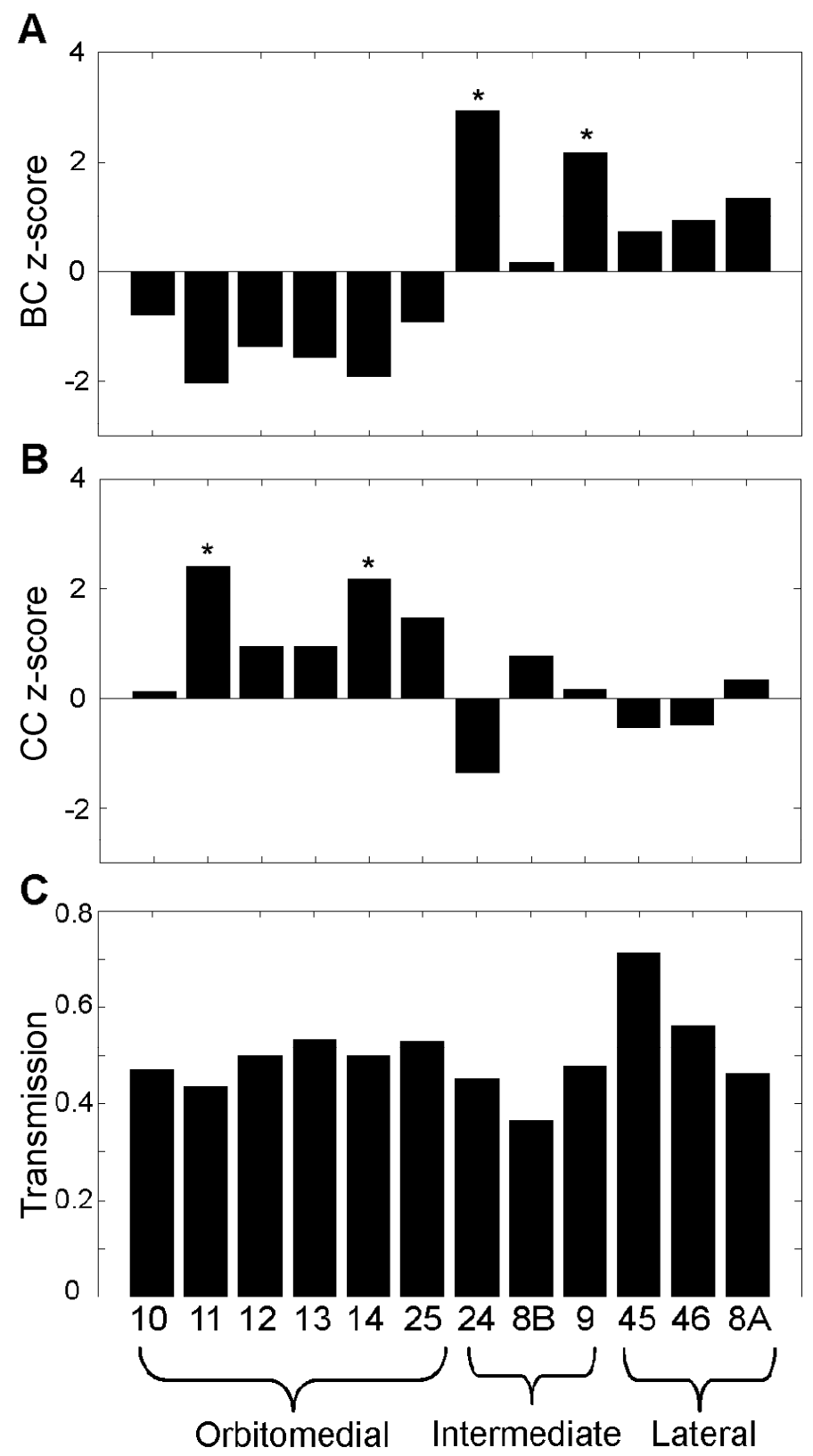

Figure 8. Summary of the network metrics for each separate PFC regions and PFC group: orbitomedial, intermediate, and lateral. A. z-scores of the $\mathrm{BC} \mathrm{B}$. CC and C. transmission index of the regions constituting each PFC group. Asterisks indicate significance ( $\mathrm{z}$-score $>2, \mathrm{p}<0.001$ ) in relation to values obtained from 1000 matched random networks. Note that these metrics were based on dataset Walker 1, with highly similar results obtained for dataset Walker 2 .

Region 25 in Walker's parcellation scheme is equivalent with Broadman's region 32 and region 32 in Barbas and Pandya, 1989 parcellation scheme (Barbas and Pandya, 1989; Kötter et al., 2001). Efferents of region 32 that target regions 46 and 10 are thought to have inhibitory and excitatory roles respectively (Medala and Barbas, 2010). Thus, hierarchical efferents, as revealed by the $\mathrm{ABH}$ optimization, of region 32 can send regulatory signals to regions 46 and 10. It is noteworthy that in our analysis region 25 is placed high in both the $\mathrm{ABH}$ and $\mathrm{LBH}$ optimization (Fig. 10) and thus its $\mathrm{ABH}$ efferents 
are FB connections (Fig. 11 A). Such features are in line with suggestions that this medial region exerts a tonic influence to lateral PFC regions. Such lateral-medial interplay might be important for multi-tasking and noise suppression in lateral PFC regions during task execution (Medala and Barbas, 2010).

Hierarchy and network metrics

The network metrics in combination with the $\mathrm{ABH}$ optimization offers additional insights about the role of each region. No significant correlations were observed between the $\mathrm{ABH}$ level of the $\mathrm{PFC}$ regions and their $\mathrm{CC}$ and $\mathrm{BC}$. This means that regions placed at higher (lower) parts of the hierarchy are not less (more) segregated/specialized, hence regions high in the hierarchy do not influence loosely connected/mutually disinterested regions (Kötter and Stephan, 2003). Additionally, the absence of correlation with the BC indicates that regions closer to the top of the hierarchy, i.e. close at the "root" of the dominant direction within the network, do not appear to be central. This argues against a "strictly centralized" PFC topology where regions that are ideally embedded to exert directed influence, i.e. placed at the top of the hierarchy, are also topologically central, i.e. exhibit high BC.

Network metrics can differentiate regions placed at the same or similar ABH level. Regions 24, 9 and 11 are placed at the bottom of the hierarchy (Fig. 5) but they exhibit different $\mathrm{CC}$ and $\mathrm{BC}$ values (Fig. $8 \mathrm{~A} \mathrm{~B}$ ). Regions 24 and 9 are the only ones with significant $\mathrm{BC}$ and hence they serve as "intermediate stations" that lie in a large fraction of the shortest paths that connect the PFC regions. This feature along with the fact that they lay at the lowest level of the hierarchy means that they integrate information through hierarchical efferents, since they are the "end point" of the dominant directed influence, and hence can resemble to "receivers" (Kötter and Stephan, 2003), and distribute information through their extensive anti-hierarchical efferents (Fig. 7). Hence, they constitute an important set of regions that facilitate the traffic of information among the PFC regions. Different techniques than the ones used here, i.e. Shapley ratings and multidimensional scaling, highlight regions 9 and 24 as important for the global PFC connectedness and the facilitation of communication of the orbitomedial and lateral PFC groups (Kötter et al., 2001, 2007). On the contrary, region 11 which is also placed at the lowest level of the hierarchy, does not exhibit a significant BC (Fig. 5 8). Instead it exhibits a significant CC (Fig. 8). Hence, region 11 appears to be part of a segregated/specialized neural circuitry and the information that it receives as the end point of the dominant directed influence, seems to directly influence a more segregated set of regions. The anti-hierarchical diagram of regions 9, 24 and 11 also reflect some of the above differences (Fig. 7). Regions 24 and 9 exhibit diffuse anti-hierarchical efferents influencing all PFC groups, while anti-hierarchical efferents of region 11 are restricted to the more segregated orbitomedial group. 

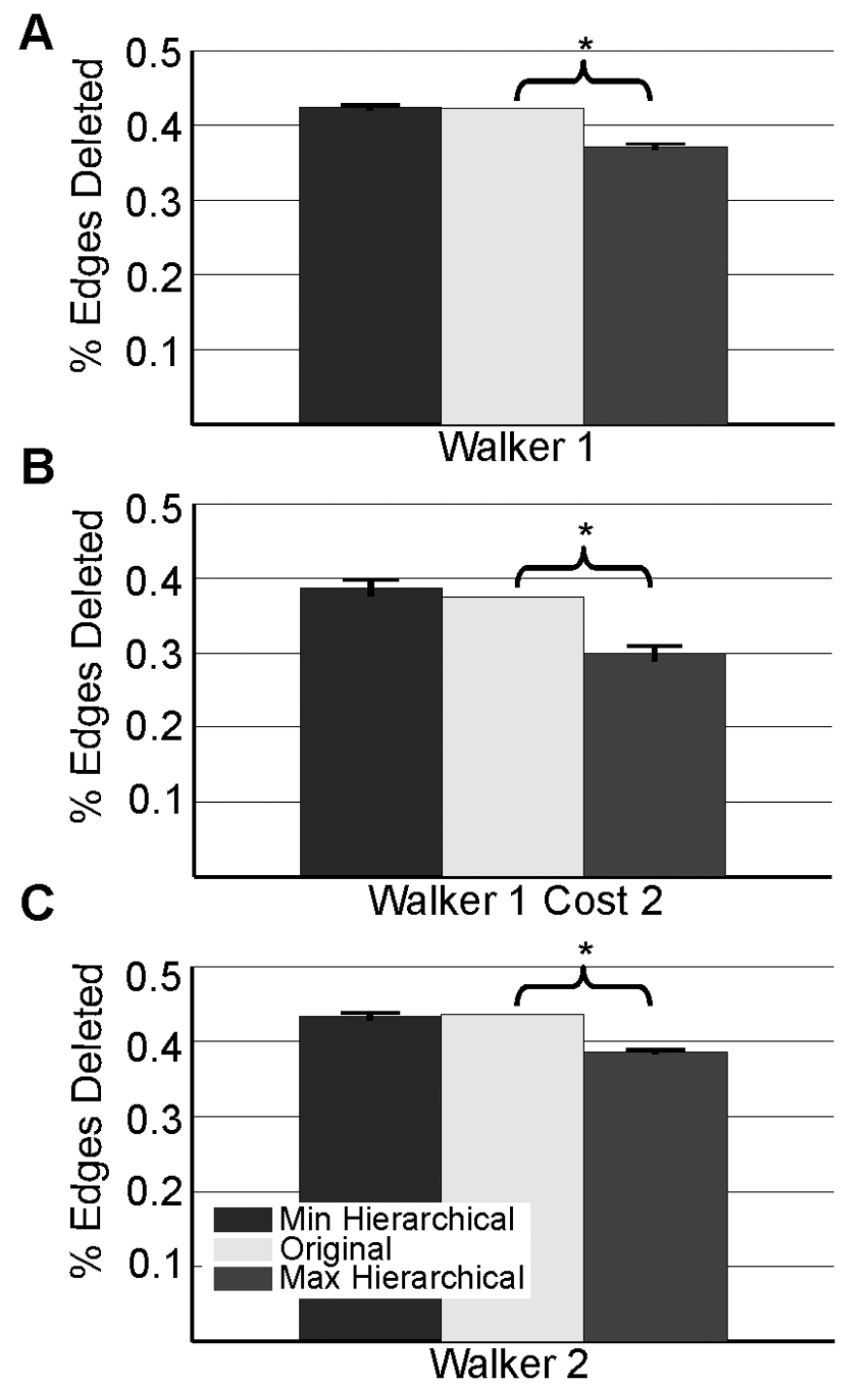

Figure 9. The PFC network exhibits overabundance of anti-hierarchical connections as defined by the ABH analysis. Comparison of the total number of edges (expressed as a percentage over the total number of edges in the network) that must be deleted in order to convert the directed graph to an acyclic one, for the original and the "maximally" and "minimally" hierarchical networks. The original network has significantly more anti-hierarchical connections than the maximally hierarchical networks but does not exhibit significant difference with the minimally hierarchical ones. Thus, the PFC topology could be considered as minimally hierarchical. Asterisks indicate significance ( $\mathrm{z}$-score $>2, \mathrm{p}<0.01)$. Note that the results hold for both datasets based on Walker's parcellation and cost functions. See sections Materials and Methods and Results for details.

\section{"Overrepresentation" of anti-hierarchical connections}

Our analysis indicates that the PFC is wired in such a way that there is an "overrepresentation" of anti-hierarchical connections (Fig. 9). The hierarchical and antihierarchical diagrams resulting from the $\mathrm{ABH}$ (Fig. 6, 7) indicate direct communication between the levels. The overrepresentation of anti-hierarchical connections might add to the multiplicity of pathways available for transmission of information from lower to higher regions. Such architecture permits communication between the hierarchical levels, not through the presence of only a few dedicated anti-hierarchical connections, but in a rather direct, decentralized and distributed way. Moreover, such features 
indicate that the PFC exhibits "overrepresentation" of anti-hierarchical connections that form loops/cycles. What can be the role subserved by such a feature? Features as such seem to be essential for the robustness of a network (Kwon and Cho, 2007). Moreover, feedback loops with excitatory and inhibitory components are important for sustained oscillations and signal amplification (Kim et al., 2008). We speculate that such features might also give rise to analogous properties when it comes to PFC function. The detailed analysis of such issues can constitute the topics of future research.

In sum, the results of the quantification of the principles of the hierarchical anteriorposterior model dictate that regardless of adopting or not the contiguity principle, the PFC is not wired in a way to "optimally" comply with the asymmetry principle dictated by the $\mathrm{ABH}$. In other words, the PFC is wired in a non-optimal way, from the view point of the asymmetry principle, since it exhibits an "overabundance" of antihierarchical connections when compared to maximally ABH networks.

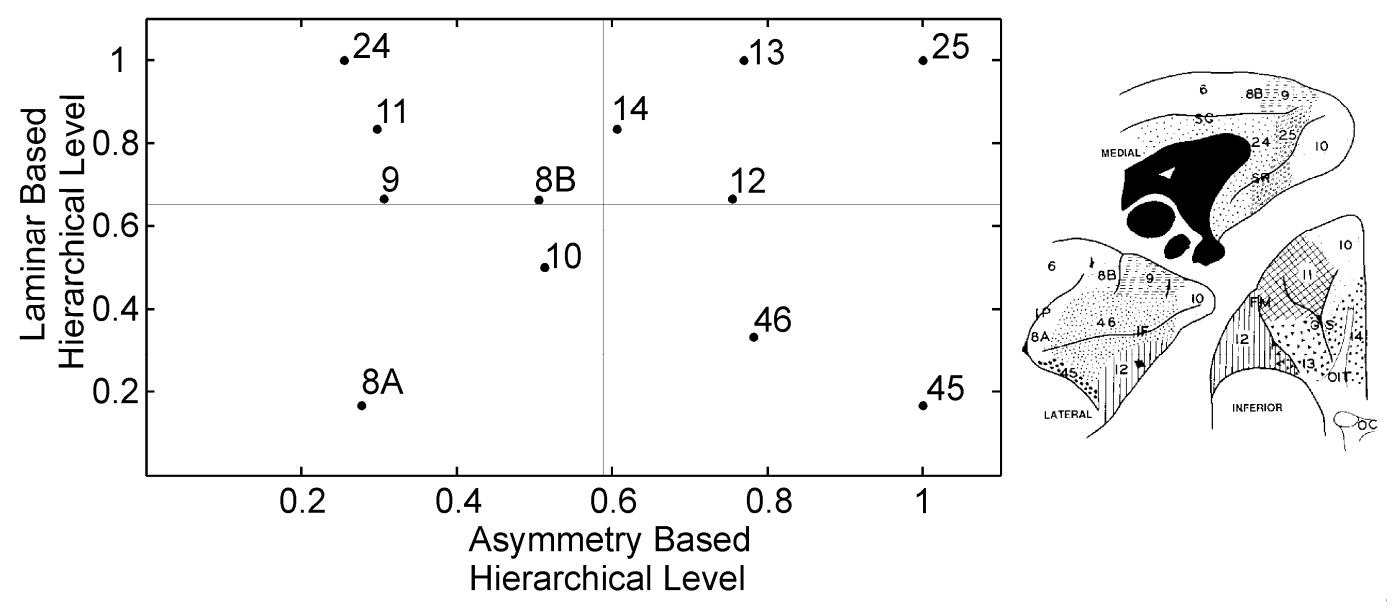

Figure 10. Scatter plot of the $\mathrm{ABH}$ and $\mathrm{LBH}$ results. The scatter plot depicts the PFC regions according to their mean $\mathrm{ABH}$ and $\mathrm{LBH}$ level. The $\mathrm{ABH}$ and $\mathrm{LBH}$ levels are scaled by dividing by the maximum value obtained during each hierarchy optimization. The planes dividing the scatterplot in 4 quartiles are centred at the mean values of the mean $\mathrm{ABH}$ and $\mathrm{LBH}$ levels. The scatterplot offers a visual inspection of the position that each PFC region occupies in both the $\mathrm{ABH}$ and $\mathrm{LBH}$ layouts and also functions as a "classification scheme". For instance, regions located in the upper right quartile occupy high levels of both ABH and LBH layouts, while regions located in the lower right quartile occupy high levels of the ABH layout but low ones of the LBH layout.

Relation between asymmetry based hierarchy and laminar based hierarchy

The $\mathrm{ABH}$ and $\mathrm{LBH}$ optimization procedures resulted in uncorrelated hierarchical layouts (Fig. 10). Instead of focusing on the "best" or more "realistic" definition of hierarchy, something that could be dictated by the pertinent questions at hand, our analyses demonstrate that the two definitions of hierarchy can be combined and offer a richer picture of the PFC connectivity.

More specifically, while our ABH analysis reveals broadcasting structures within the PFC, along the lines of findings from previous analysis (Kötter et al., 2001), the LBH results reveal additional information about the regions that function as broadcasters and receivers. The $\mathrm{ABH}$ designates regions 25, 45, 46 and 13 as broadcasters and thus are more influencing rather than getting influenced by other PFC regions. The dominant 
exerted influence of regions 25 and 13, through their hierarchical connections revealed by the ABH optimization, involves FB connections, while that of region 45 involves FF connections. Region 46 exhibits both FF and FB connections with a prevalence of FF ones (Fig. $11 \mathrm{~A}$ ). The differentiation of these regions is also reflected in the fact that they are located in different quartiles of the ABH and LBH plot (Fig. 10). Hence, while all regions are broadcasters, their efferents target preferentially different laminae and might have different impact on the dynamics of the PFC microcircuit. The exerted influence from regions 25 and 46 target predominantly supragranular layers where calbindin positive neurons are primarily located and target distant dendrites of pyramidal neurons (Barbas and Hilgetag, 2002). On the contrary, the exerted influence from region 45 is characterized by FF connections and thus might mostly interact with another class of neurons i.e., presumed inhibitory parvalbumin positive interneurons, primarily located in middle layers, targeting proximal pyramidal neurons (Barbas and Hilgetag, 2002). These connections appear to have different functional roles and might be important for the co-ordination of the intrinsic and associational neural circuitry involving PFC regions (Pucak et al., 1996; Barbas and Zikopoulos, 2007). Such interpretations are rendered possible by combining the results from the $\mathrm{ABH}$ and $\mathrm{LBH}$. Assigning LB values to efferents that were deemed hierarchical (Fig. $11 \mathrm{~A}$ ) or antihierarchical (Fig. $11 \mathrm{~B}$ ) by the $\mathrm{ABH}$ optimization offers hints for the role of the efferents that constitute the dominant direction, or go against it, within the PFC. We should note that despite that the laminar termination of a connection is informative about its role, additional analysis is needed in order to decipher the exact synaptic targets of a FF or FB connection. For instance, connections from region 32 to regions 10 and 46 terminate predominantly to layers I-III, and thus constitute FB connections, but there is a higher number of synapses with presumed inhibitory neurons associated with the connections from region 32 to 46 when compared with the ones from region 32 to 10 (Medala and Barbas, 2010). Hence, quantitative histological data are needed for uncovering the exact synaptic targets of a connection and inferring a more precise functional role of FB (or FF) PFC connections. 

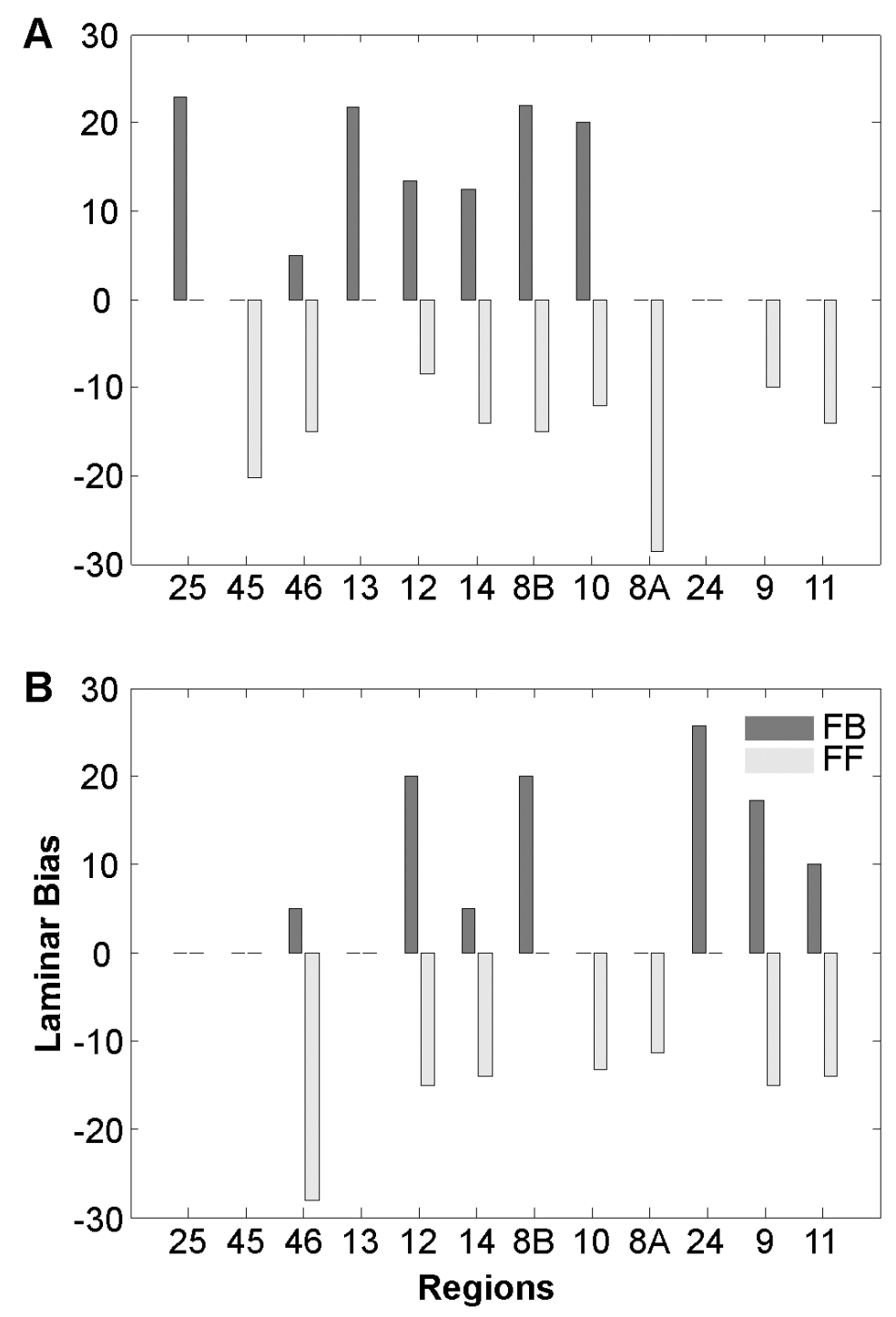

Figure 11. Summary of the laminar bias index of the efferents that were more frequently hierarchical or antihierarchical during the $\mathrm{ABH}$ optimization. Mean laminar bias index for A. The most frequent hierarchical efferents (see Fig. 6) and B. The most frequent anti-hierarchical efferents (see Fig. 7) of each PFC region. The PFC regions are arranged from left to right with a decreasing order of hierarchy based on the ABH results with dataset Walker 1 cost function 1 (see Fig. 5 A). The mean laminar index was calculated separately for positive (FB) and negative values (FF). These values indicate the prelevance of FF or FB type of connections, or an intermediate profile, for the most frequent hierarchical and anti-hierarchical connections, and thus highlight their potential diverge role in PFC communication (see Discussion for details).

Moreover, the results from $\mathrm{ABH}$ and $\mathrm{LBH}$ can be used for developing complementary hypothesis and predictions related to functional studies, e.g. electrophysiology in multiple macaque PFC regions. More specifically, the $\mathrm{ABH}$ that quantifies the dominant direction within the structural PFC network can be used for assessing which regions are anatomically favoured to exhibit directed influence during a task, i.e. effective connectivity. Regions in high $\mathrm{ABH}$ levels seem more suitable candidates to function as the regions around which more "outflow" effective connectivity configurations can be articulated. Hence, $\mathrm{ABH}$ is informative about the directionality of the flow of information. LBH can be informative about the way that the dialogue between the PFC 


\section{Chapter 3}

regions takes place. Laminar based recordings from the macaque V1 reveals a preference of gamma activity in upper layers (Maier et al., 2010). Additionally, effective connectivity from V1 to V4 is primarily observed in the gamma band whereas effective connectivity from V4 to V1 does not show such a gamma preference (Bosman et al., 2012). V1 is located lower than V4 in the LBH hierarchy of the visual system and thus V1 to V4 connections can be considered as FF and connections from V4 to V1 as FB (Hilgetag et al., 2000). FF connections predominantly originate from upper layers whereas FB from lower layers (Felleman and Van Essen, 1991). Hence, it is hypothesized that the functional dialogue between regions of the visual system is shaped from the laminar patterns of their connections, determining the frequency bandwidth that the two regions "use" for exerting influence to each other (Bosman et al., 2012). In other words, the preference of gamma band for upper layers in conjunction with the laminar origin of FF and FB connections is reflected in the functional dialogue between V1 and V4. These structure-function relations might also hold for the PFC. Our LBH results can be used for generating hypothesis for the functional dialogue among the PFC regions, e.g. the frequency bandwidth preferences of inter-regional influences. Such investigation is important since the demonstration of a comparable structure-function correspondance for the PFC, as the aforementioned preliminary evidence suggest for the visual system, would suggest a general structure-function relation pertaining to the macaque cerebral cortex as a whole. In sum, ABH and LBH offer distinct layouts of the anatomical skeleton of the macaque PFC that are useful for predictions and hypothesis of distinct functional aspects of the PFC.

With respect to the hierarchical anterior-posterior model, while the $\mathrm{ABH}$ results do not seem to support its principles and predictions, the LBH results reveal that region 10 is located in higher levels than more posterior regions, i.e. 46, 45, 8A, as expected from the differences of their structural types. This suggests that a $\mathrm{LBH}$ is a more plausible working hypothesis for PFC models advocating an anterior-posterior hierarchical gradient that is based on the anatomy of the PFC.

Finally, we should note that the classification of connections as FF and FB constitutes a broad distinction and can mask out further fine grained differentiations and modes of communication (Rockland, 2004). Such differences can include subdifferentiations of FB connections based on the caliber size of the axons and the size of the buttons, features that can give rise to different degrees of conduction velocity and efficiency (Rockland, 2004; Medala and Barbas, 2010).

Does a structural hierarchical architecture imply a functional hierarchical architecture?

Despite that evidence from empirical and modelling studies suggest that the way a region is embedded within a structural network constraints its function (Kötter and Sommer, 2000; Passingham et al., 2002; Smith et al., 2006) this relation is far from a simple 1:1 mapping. For instance, while the anatomical architecture of the visual system is hierarchical (Hilgetag et al., 2000) computational modelling demonstrates that functional properties, i.e. neural response latencies to visual stimulation, are not sequential and thus an anatomical hierarchy does not necessarily entail a functional hierarchy (Petroni et al., 2001). Moreover, structure-function deviations are evident in the discrepancy between the structural hierarchy and the signal timing of the regions of the visual system (Schmolesky et al., 1998; Capalbo et al., 2008).

An analogous picture can also hold for the PFC and hierarchical diagrams obtained from structural connectivity might not coincide with the flow of information during the 
execution of a task (Hegdé and Felleman, 2007). Such structure-function mismatches seem reasonable when considering findings illustrating the dynamic nature of interactions of brain regions modulated by context and task (Rowe et al., 2005). Additionally, various "functional motifs" can be embedded within a "structural skeleton" of a brain structure, and each functional motif can arise at a particular context or phase of a task (Sporns and Kötter, 2004). Hence, a structural hierarchical architecture does not necessarily entail a functional hierarchical architecture, nor does the existence of a particular orientation along an axis, e.g. anterior-posterior, of a structural hierarchy, or non-optimal hierarchy, entails the same orientation for a possible functional hierarchy. Ultimately, structural connectivity estimates should be combined with functional measurements (see e.g. Smith et al., 2006; Vanduffel et al., 1997) from the same brain in order to examine the PFC architecture at multiple levels and be able to unravel the rules determining structure-function correspondences and deviations.

\section{Limitations and perspectives}

While currently we examined the PFC as a whole, future analyses can focus on a specific surface. To this end, more fine grained parcellation schemes can be adopted, instead of the rather coarse Walker scheme (e.g. Petrides and Pandya, 1994; Carmichael and Price, 1996). Such fine grained parcellations might be more suitable for the examination of the PFC organization not only along the anterior-posterior but also the medial-lateral or dorsal-medial axis (O'Reilly, 2010).

In the current study we only considered PFC regions and premotor and motor regions (e.g. BA 6 and 4) were not included in the analysis. This exclusion allowed us to focus on the PFC and exploit the rich published datasets for this brain structure of the macaque. Future analysis can investigate the PFC within a broader context by including fronto-parietal, fronto-temporal and fronto-thalamic connections.

An intriguing future question is if and how the ABH PFC hierarchical layout is related to architectonic/developmental features of the PFC regions. For instance, the ABH level of a region could be related to its ontogeny. Early development in ontogeny of the ACC favours the diffuse and extensive establishment of its connections (Zikopoulos and Barbas, 2010). Hence, such regions could be favoured to evolve into a broadcasting structure, i.e. a highly influential region with extensive connections. Future studies could e.g. correlate the $\mathrm{ABH}$ level of each $\mathrm{PFC}$ region with the corresponding onset in ontogeny in order unveil a potential link. Moreover, an interesting future direction would be to relate the hierarchy results and phylogenetic characteristics of the PFC regions. The gradations from the two primordial moieties, i.e. archicortex and paleocortex, forming the basoventral and dorsomedial trends (Pandya \& Yeterian, 1990) have also been interpreted within an evolutionary context (Sanides, 1964; but see Preus $\&$ Goldman-Rakic, 1991). Hence, the dominant direction within the PFC, as revealed by the $\mathrm{ABH}$, could be related with the direction of the aforementioned gradations, and consequently, the progression from older to newer phylogenetic regions. Since the LBH optimization is highly dependent on the direction of gradations of the basoventral and dorsomedial trends, i.e. the progressive eulamination of PFC regions (Barbas and Rempel-Clower, 1997), the resulting hierarchical levels are closely linked to such direction. This in turn, in conjunction with the fact that the $\mathrm{ABH}$ and $\mathrm{LBH}$ results do not coincide, would mean that the $\mathrm{ABH}$ arrangements of the $\mathrm{PFC}$ regions do not relate to the direction of the gradations. The explicit quantification of the relation of the $\mathrm{ABH}$ 


\section{Chapter 3}

and LBH results with ontogeny and phylogeny features constitutes a potential future research topic.

While many meaningful cost functions can be used during the optimization procedure (Krumnack et al., 2010; Hilgetag et al., 2000), we currently used these particular cost functions for the $\mathrm{ABH}$ optimization since they correspond to the principles of the hierarchical anterior-posterior model. Despite that the results were not identical, the main conclusions of the study are supported by the results of both cost functions and different data sets (Table 3). In the future, other cost functions that e.g. penalize connections that cross many hierarchical levels can be adopted. Since the strength of the connections between the PFC regions is graded, cost functions that employ weighted information can also be adopted. We employed a cost function that takes into account the weight of the anti-hierarchical connections and obtained highly similar results with the other cost functions (data not shown). Hence, decisions on graded versus binary connectivity do not influence the current $\mathrm{ABH}$ results.

The LBH optimization was based on estimated values of \% AL IV-VI by using the Delta index as predictor. Since the values of \% AL IV-VI and the Delta index are characterized by an almost perfect correlation, in principle the hierarchical arrangement could also be based on the Delta index. In the current study we used the values of \% AL IV-VI for the hierarchical optimization in order to directly link the approach with laminar based hierarchical arrangements previously applied to other parts of the cortex, e.g. visual cortex (Hilgetag and Grant, 2010) and illustrate how a laminar based hierarchical arrangement of the PFC regions can be performed in the future by using directly the region to region \% AL IV-VI values that might slightly deviate from the ones estimated from the Delta index. Since the Delta index is the best predictor of laminar patterns of connections (Hilgetag and Grant, 2010), we predict that such an arrangement will be highly similar with the one obtained in our study.

Despite similarities between the PFC of human and non-human primates (Ongür and Price, 2000; Petrides et al., 2012) differences also exist. Interestingly, pronounced differences are reported for region 10 which became enlarged and more specialized in humans (Semendeferi et al., 2001). Such potential changes might have an impact on the wiring of this region and affect its hierarchical role within the human PFC.

Lastly, the analytic tools employed can be used for uncovering similarities in the topologies of the structural networks of e.g. the PFC of rodents and higher primates in order to assess preservation of the roles of putative homologous regions (see e.g. Uylings and Van Eden, 1990).

\section{Conclusions}

In sum, we have examined the structural connectivity of the macaque PFC by using tools from network science and an exhaustive dataset. On the one hand, the principles and predictions of the $\mathrm{ABH}$ do not support the connectivity principles of the hierarchical anterior-posterior model. On the other hand, the LBH seem to be a more tenable hypothesis for models incorporating evidence on the connectivity of the macaque PFC and advocating a hierarchical anterior-posterior gradient.

Approaches as the current one can inform models of the human PFC and highlight the power of formal analysis for the investigation of PFC organization. Such approaches can be helpful for understanding how integrated behaviour is accomplished and what structural alterations might underlie mental diseases. 


\section{Chapter 3}

\section{$\underline{\text { References }}$}

Averbeck BB, Seo M (2008) The statistical neuroanatomy of frontal networks in the macaque. PLoS Comput Biol 4:e1000050.

Badre D, D’Esposito M (2007) Functional magnetic resonance imaging evidence for a hierarchical organization of the prefrontal cortex. J Cogn Neurosci 19:2082-99.

Badre D, D'Esposito M (2009) Is the rostro-caudal axis of the frontal lobe hierarchical? Nat Rev Neurosci. 10:659-69.

Barbas H, Pandya DN (1989) Architecture and intrinsic connections of the prefrontal cortex in the rhesus monkey. J Comp Neurol 286:353-375.

Barbas H (1995) Anatomic basis of cognitive-emotional interactions in the primate prefrontal cortex. Neurosci and Biobehav Rev 19:499-510.

Barbas H, Rempel-Clower N (1997) Cortical structure predicts the pattern of corticocortical connections. Cereb Cortex 7:635-646.

Barbas H (2000) Connections underlying the synthesis of cognition, memory, and emotion in primate prefrontal cortices. Brain Res 52:319 -330.

Barbas H, Hilgetag CC (2002) Rules relating connections to cortical structure in primate prefrontal cortex. Neurocomputing 44-46:301-308.

Barbas H (2007) Specialized elements of orbitofrontal cortex in primates. Ann NY Acad Sci 1121:10-32.

Barbas H, Zikopoulos B (2007) The prefrontal cortex and flexible behavior. The Neuroscientist 13:532-545.

Bond AH (2004) An information-processing analysis of the functional architecture of the primate neocortex. J Theor Biol 227:51-79.

Bosman CA, Schoffelen J-M, Brunet N, Oostenveld R, Bastos AM, Womelsdorf T, Rubehn B, Stieglitz T, De Weerd P, Fries P (2012) Attentional stimulus selection through selective synchronization between monkey visual areas. Neuron 75:875-888.

Capalbo M, Postma E, Goebel R (2008) Combining structural connectivity and response latencies to model the structure of the visual system. PLoS Comput Biol 4:e1000159.

Carmichael, ST, Price JL (1994) Architectonic subdivision of the orbital and medial prefrontal cortex in the macaque monkey. J Comp Neurol 346: 366-402.

Carmichael ST, Price JL (1996) Connectional networks within the orbital and medial prefrontal cortex of macaque monkeys. J Comp Neurol 371:179-207.

Dombrowski SM, Hilgetag CC, Barbas H (2001) Quantitative architecture distinguishes prefrontal cortical systems in the rhesus monkey. Cereb Cortex 11:975-88.

Eades P, Lin X, Smyth WF (1993) A fast and effective heuristic for the feedback arc set problem. Inform Process Lett 47:319-323.

Felleman DJ, Van Essen DC (1991) Distributed hierarchical processing in the primate cerebral cortex. Cereb Cortex 1:1-47.

Ghashghaei HT, Hilgetag CC, Barbas H (2007) Sequence of information processing for emotions based on the anatomic dialogue between prefrontal cortex and amygdala. NeuroImage 34:905-23.

Groenewegen HJ, Uylings HBM (2000) The prefrontal cortex and the integration of sensory, limbic and autonomic information. In: Cognition, emotion and autonomic responses: The integrative role of the prefrontal cortex and limbic structures. Progress in brain research vol.126. (HBM Uylings CG van Eden JPC de Bruin MGP Feenstra CMA Pennartz eds) Amsterdam Elsevier. p.3-19. 


\section{Chapter 3}

Hegdé J, Felleman DJ (2007) Reappraising the functional implications of the primate visual anatomical hierarchy. The Neuroscientist 13(5):416-421.

Hilgetag CC, O’Neill M Young MP (1996) Indeterminate organization of the visual system. Science 271:7767.

Hilgetag CC, O’Neill M, Young MP (2000) Hierarchical organization of macaque and cat cortical sensory systems explored with a novel network processor. Philos Trans R Soc Lond B Biol Sci 355:71-89.

Hilgetag CC, Kötter R, Stephan KE, Sporns O (2002) Computational methods for the analysis of brain connectivity, In: Computational Neuroanatomy - Principles and methods (Ascoli GA Ed.) Humana Press, p. 295-335.

Hilgetag CC, Grant S. 2010. Cytoarchitectural differences are a key determinant of laminar projection origins in the visual cortex. NeuroImage 51:1006-17.

Honey CJ, Kötter R, Breakspear M, Sporns O (2007) Network structure of cerebral cortex shapes functional connectivity on multiple time scales. Proc Natl Acad Sci U S A 104:10240-5.

Ispolatov I, Maslov S (2008) Detection of the dominant direction of information flow and feedback links in densely interconnected regulatory networks. BMC Bioinformatics 9:424.

Kaiser M, Hilgetag CC (2006) Nonoptimal component placement, but short processing paths, due to longdistance projections in neural systems. PLoS Comput Biol 2:e95.

Kim JR, Yoon Y, Cho KH (2008) Coupled feedback loops form dynamic motifs of cellular networks. Biophys J 94:359-365.

Kirkpatrick S, Gelatt CD, Vecchi MP (1983) Optimization by simulated annealing. Science 220:671-680.

Koechlin E, Ody C, Kouneiher F (2003) The architecture of cognitive control in the human prefrontal cortex. Science 302:1181-5.

Koechlin E, Summerfield C (2007) An information theoretical approach to prefrontal executive function. Trends Cogn Sci 11:229-35.

Kouneiher F, Charron S, Koechlin E (2009) Motivation and cognitive control in the human prefrontal cortex. Nat Neurosci 12:939-45.

Kötter R, Sommer FT (2000) Global relationship between anatomical connectivity and activity propagation in the cerebral cortex. Philos Trans R Soc Lond B Biol Sci 355:127-34.

Kötter R (2001) Connectional characteristics of areas in Walker's map of primate prefrontal cortex. Neurocomputing 38-40:741-746.

Kötter R, Stephan KE (2003) Network participation indices: characterizing component roles for information processing in neural networks. Neural Networks 16:1261-75.

Kötter R (2004) Online retrieval, processing, and visualization of primate connectivity data from the CoCoMac Database. Neuroinformatics 2:127:144.

Kötter R, Reid AT, Krumnack A, Wanke E, Sporns O (2007) Shapley ratings in brain networks. Front Neuroinf 1:1-9.

Krumnack A, Reid AT, Wanke E, Bezgin G, Kötter R (2010) Criteria for optimizing cortical hierarchies with continuous ranges. Front Neuroinf 4:7. 
Kwon YK, Cho KH (2007) Analysis of feedback loops and robustness in network evolution based on Boolean models. BMC Bioinformatics 9:1-9.

Maier A, Adams GK, Aura C, Leopold DA, Radman T, Kline N, Roberts M (2010) Distinct superficial and deep laminar domains of activity in the visual cortex during rest and stimulation. Front Syst Neurosci 4:1-11.

Maslov S, Sneppen K, Zaliznyak A (2007) Detection of topological patterns in complex networks: Correlation profile of the Internet. Physica A. 333:529-540.

Medalla M, Barbas H (2010) Anterior cingulate synapses in prefrontal areas 10 and 46 suggest differential influence in cognitive control. J Neurosci 30:16068-81.

Metropolis N, Rosenbluth AW, Rosenbluth MN, Teller AH, Teller E (1953) Equation of state equations by fast computing machines. J. Chem. Phys.21:1087-1092.

Miller EK, Cohen JD (2001) An integrative theory of prefrontal cortex function. Ann Rev Neurosci 24:167202.

Modha DS, Singh R (2010) Network architecture of the long-distance pathways in the macaque brain. Proc Natl Acad Sci U S A 107:13485-90.

Morecraft RJ, Geula C, Mesulam M (1992) Cytoarchitecture and neural afferents of orbitofrontal cortex in the brain of the monkey. J Comp Neurol 358:341-358.

Murray EA (2007) The amygdala, reward and emotion. Trends Cogn Sci 11:489-497.

Ongür D, Price JL (2000) The organization of networks within the orbital and medial prefrontal cortex of rats, monkeys and humans. Cereb Cortex 10:206-19.

O’Reilly RC (2010) The What and How of prefrontal cortical organization. Trends Neurosci 33:355-61.

Pandya DN, Yeterian EH (1990) Prefrontal cortex in relation to other cortical areas in rhesus monkey: architecture and connections. In: The prefrontal cortex: its structure, function and pathology. Progress in brain research vol. 85. (HBM Uylings CG van Eden JPC de Bruin MGP Feenstra CMA Pennartz Eds) Amsterdam Elsevier. p.63-94.

Passingham RE, Stephan KE, Kötter R (2002) The anatomical basis of functional localization in the cortex. Nat Rev Neurosci 3:606-16.

Petroni, F, Panzeri, S, Hilgetag, CC, Scannell, JW, Kötter, R, Young, MP (2001) Hierarchical organisation and neuronal response latencies in the primate visual system. Neurocomputing 38-40:1519-1523.

Petrides M, Pandya, DN (1994) Comparative architectonic analysis of the human and the macaque frontal cortex. In: Handbook of neuropsychology (F. Boller J. Grafman eds), vol. 9, Amsterdam Elsevier. p. 17-58.

Petrides M, Tomaiuolo F, Yeterian EH, Pandya DN (2012) The prefrontal cortex: Comparative architectonic organization in the human and the macaque monkey brains. Cortex 48:46-57.

Preuss TM, Goldman-Rakic PS (1991) Myelo- and cytoarchitecture of the granular frontal cortex and surrounding regions in the strepsirhine primate Galago and the anthropoid primate Macaca. J Comp Neurol 310:429-74.

Pucak ML, Levitt JB, Lund JS, Lewis DA (1996) Patterns of intrinsic and associational circuitry in monkey prefrontal cortex. J Comp Neurol 376: 614-630.

Rockland K (2004) Feedback connections: splitting the arrow In: The Primate Visual System (Kaas JH, Collins CE Eds.) CRC Press, p. 387-405. 


\section{Chapter 3}

Rowe JB, Stephan KE, Friston K, Frackowiak RSJ, Passingham RE (2005) The prefrontal cortex shows context- specific changes in effective connectivity to motor or visual cortex during the selection of action or colour. Cereb Cortex 15:85-95.

Rubinov M, Sporns O (2010) Complex network measures of brain connectivity: uses and interpretations. NeuroImage 52:1059-69.

Sanides F (1964) The cyto-myeloarchitecture of the human frontal lobe and its relation to phylogenetic differentiation of the cerebral cortex. J. Hirnforsch 6:269-282.

Schmolesky MT, Wang Y, Hanes DP, Thompson KG, Schall JD, Leventhal AG (1998) Signal timing across the macaque visual system J Neurophysiol 79:3272-3278.

Semendeferi K, Armstrong E, Schleicher A, Zilles K, Van Hoesen GW (2001) Prefrontal cortex in humans and apes: a comparative study of area 10. Am J Phys Anthro 114:224-41.

Smith VA, Yu J, Smulders TV, Hartemink AJ, Jarvis ED (2006) Computational inference of neural information flow networks. PLoS Comput Biol 2:e161.

Soranzo N, Ramezani F, Iacono G, Altafini C (2012) Decompositions of large-scale biological systems based on dynamical properties. Bioinformatics 28:76-83.

Sporns O, Kötter R (2004) Motifs in brain networks. PloS Biol 2:e369.

Sporns O, Honey CJ, Kötter R (2007) Identification and classification of hubs in brain networks. PloS One 2:e1049.

Stephan KE, Zilles K, Kötter R (2000) Coordinate-independent mapping of structural and functional data by objective relational transformation (ORT). Philos Trans R Soc Lond B Biol Sci 355:37-54.

Stephan KE, Kamper L, Bozkurt A, Burns GAPC, Young MP, Kötter R (2001) Advanced database methodology for the collation of connectivity data on the macaque brain (CoCoMac). Philos Trans R Soc Lond B Biol Sci 356:1159-1186.

Suzuki W, Amaral DG (1994) Perirhinal and parahippocampal cortices of the macaque monkey: cortical afferents. J Comp Neurol 350:497-533.

Taren A, Venkatraman V, Huettel S (2011) A parallel functional topography between medial and lateral prefrontal cortex: evidence and implications for cognitive control. J Neurosci 31:5026-31.

Uylings HBM and Van Eden, CG (1990) Qualitative and quantitative comparison of the prefrontal cortices in rat and in primates, including humans. Prog. Brain Res 85:31-62.

Uylings HBM, Rajkowska G, Sanz-Arigita E, Amunts K, Zilles K (2005) Consequences of large interindividual variability for human brain atlases: converging macroscopical imaging and microscopical neuroanatomy. Anat Embryol 210:423-31.

Vanduffel W, Payne BR, Lomber SG, Orban GA (1997) Functional impact of cerebral connections Proc Natl Acad Sci U S A 94:7617-7620.

Walker AE (1940) A cytoarchitectural study of the prefrontal area of the macaque monkey. J Comp Neurol 73: 59-86.

Zikopoulos B, Barbas H (2010) Changes in prefrontal axons may disrupt the network in autism. J Neurosci 30:14595-14609. 


\section{Supplementary material}

Table S1. ABH optimization for Modha \& Singh PFC

\begin{tabular}{|c|c|c|}
\hline PFC region & Hierachical level & Frequency of assignement \\
\hline 12 & 14 & 0,958 \\
\hline $45 \mathrm{~B}$ & 14 & 0,955 \\
\hline 45 & 14 & 0,653 \\
\hline $\mathrm{Gu}$ & 14 & 0,451 \\
\hline OFap & 14 & 0,299 \\
\hline 8 & 14 & 0,250 \\
\hline $46 \mathrm{vr}$ & 14 & 0,219 \\
\hline $46 \mathrm{v}$ & 13 & 0,908 \\
\hline 46 & 13 & 0,903 \\
\hline $10 \mathrm{~m}$ & 13 & 0,377 \\
\hline PFCorb & 13 & 0,321 \\
\hline 13 & 12 & 0,670 \\
\hline $13 a$ & 12 & 0,385 \\
\hline 9 & 11 & 0,431 \\
\hline 32 & 11 & 0,231 \\
\hline $45 \mathrm{~A}$ & 11 & 0,137 \\
\hline FEF & 10 & 0,089 \\
\hline ProM\#2 & 9 & 0,148 \\
\hline 10 & 8 & 0,347 \\
\hline 120 & 8 & 0,339 \\
\hline $46 \mathrm{~d}$ & 8 & 0,332 \\
\hline $12 \mathrm{~m}$ & 8 & 0,198 \\
\hline $11 \mathrm{~m}$ & 7 & 0,277 \\
\hline $13 \mathrm{M}$ & 7 & 0,209 \\
\hline $10 \mathrm{~d}$ & 7 & 0,103 \\
\hline $46 \mathrm{dr}$ & 7 & 0,094 \\
\hline 14 & 6 & 0,564 \\
\hline $47 / 12$ & 6 & 0,367 \\
\hline $10 v$ & 6 & 0,329 \\
\hline PS & 6 & 0,139 \\
\hline $8 \mathrm{~B}$ & 5 & 0,649 \\
\hline 100 & 5 & 0,500 \\
\hline $12 r$ & 5 & 0,474 \\
\hline $46 f$ & 5 & 0,089 \\
\hline 11 & 4 & 0,709 \\
\hline 111 & 4 & 0,518 \\
\hline 121 & 3 & 0,730 \\
\hline $9 / 46 \mathrm{v}$ & 3 & 0,490 \\
\hline 140 & 3 & 0,168 \\
\hline $14 r$ & 2 & 0,733 \\
\hline M9 & 2 & 0,480 \\
\hline $9 / 46 d$ & 2 & 0,403 \\
\hline $13 \mathrm{~L}$ & 1 & 0,843 \\
\hline $8 \mathrm{Ad}$ & 1 & 0,696 \\
\hline D9 & 1 & 0,479 \\
\hline $8 \mathrm{~A}$ & 1 & 0,299 \\
\hline L9 & 1 & 0,197 \\
\hline
\end{tabular}




\section{Chapter 3}

(Table S1) Hierarchical arrangement of the PFC regions. Regions are sorted according to their hierarchical level (high to low) and within each level according to their frequency of assignment (high to low). Note that region 10 and its subdivisions, with the exception of $10 \mathrm{~m}$, do not occupy high levels of the hierarchy and is surpassed by more posterior regions e.g. 45B, 46. The number of levels was selected empirically (see main text Materials and Methods). For information on the acronyms used for the PFC regions see Modha \& Singh, 2010 .

Table S2. ABH optimization for lateral regions + region 10 for dataset Walker 1

\begin{tabular}{|c|c|c|}
\hline PFC region & Hierarchical level & Frequency of assignement \\
\hline 45 & 4 & 0,388 \\
\hline $8 \mathrm{~A}$ & 4 & 0,263 \\
\hline 46 & 3 & 0,387 \\
\hline 10 & 1 & 0,750 \\
\hline
\end{tabular}

Selection of a subset of PFC regions (lateral +10 ) and the application of the hierarchy optimization illustrate that no anterior-posterior gradient is observed with region 10 on top of the hierarchy. The hierarchy optimization was performed with cost function 1, but similar results were obtained with cost function 2 . The number of levels was selected empirically (see main text Materials and Methods). Arrangement of regions as described in Table S1. 
Table S3. ABH optimization for lateral regions for dataset Modha \& Singh PFC

\begin{tabular}{|c|c|c|}
\hline $\begin{array}{c}\text { PFC } \\
\text { regions }\end{array}$ & Hierarchical level & Frequency of assignement \\
\hline $45 \mathrm{~B}$ & 5 & 1 \\
\hline 45 & 5 & 0,618 \\
\hline $45 \mathrm{~A}$ & 5 & 0,513 \\
\hline $46 \mathrm{~d}$ & 5 & 0,47 \\
\hline FEF & 5 & 0,266 \\
\hline $46 \mathrm{f}$ & 5 & 0,239 \\
\hline $46 \mathrm{dr}$ & 5 & 0,228 \\
\hline 46 & 4 & 1 \\
\hline $46 \mathrm{v}$ & 4 & 0,545 \\
\hline $9 / 46 \mathrm{v}$ & 4 & 0,469 \\
\hline $10 \mathrm{v}$ & 3 & 1 \\
\hline $10 \mathrm{~d}$ & 3 & 0,344 \\
\hline 121 & 2 & 1 \\
\hline $8 \mathrm{~A}$ & 2 & 0,51 \\
\hline L9 & 2 & 0,38 \\
\hline $9 / 46 \mathrm{~d}$ & 2 & 0,319 \\
\hline D9 & 1 & 1 \\
\hline $12 \mathrm{r}$ & 1 & 0,695 \\
\hline $8 \mathrm{Ad}$ & 1 & 0,508 \\
\hline PS & 1 & 0,347 \\
\hline
\end{tabular}

Selection of only the lateral PFC regions and the application of the hierarchy optimization illustrate that no anterior-posterior gradient is observed with lateral subdivisions of region 10 on top of the hierarchy. The number of levels was selected empirically (see main text Materials and Methods). Arrangement of regions as described in Table S1. 


\section{Chapter 3}

Table S4. Structural type values for each region of Walker's parcellation scheme.

\begin{tabular}{|c|c|}
\hline Walker Region & Structural Type \\
\hline 10 & 4 \\
\hline 11 & 3 \\
\hline 12 & 3,5 \\
\hline 13 & 2 \\
\hline 14 & 3 \\
\hline 24 & 2 \\
\hline 25 & 2 \\
\hline 45 & 5 \\
\hline 46 & 4,5 \\
\hline $8 \mathrm{~A}$ & 5 \\
\hline $8 \mathrm{~B}$ & 3,5 \\
\hline 9 & 3,5 \\
\hline
\end{tabular}

Each region of Walker's parcellation scheme was matched to a region of the (Barbas \& Pandya, 1989) parcellation. Subsequently, a structural type number was assigned according to the structural model described in (Barbas \& Rempel-Clower, 1997).

Table S5. LBH optimization results.

\begin{tabular}{|c|c|}
\hline Walker regions & Hierarchical level \\
\hline 13 & 6 \\
\hline 24 & 6 \\
\hline 25 & 6 \\
\hline 11 & 5 \\
\hline 12 & 4 \\
\hline 9 & 4 \\
\hline $8 \mathrm{~B}$ & 3,98 \\
\hline 10 & 3 \\
\hline 46 & 2 \\
\hline 45 & 1 \\
\hline $8 \mathrm{~A}$ & 1 \\
\hline
\end{tabular}

Walker regions are arranged in descending order (top to bottom) according to the hierarchical level that the LBH optimization assigned. 


\section{Chapter 3}

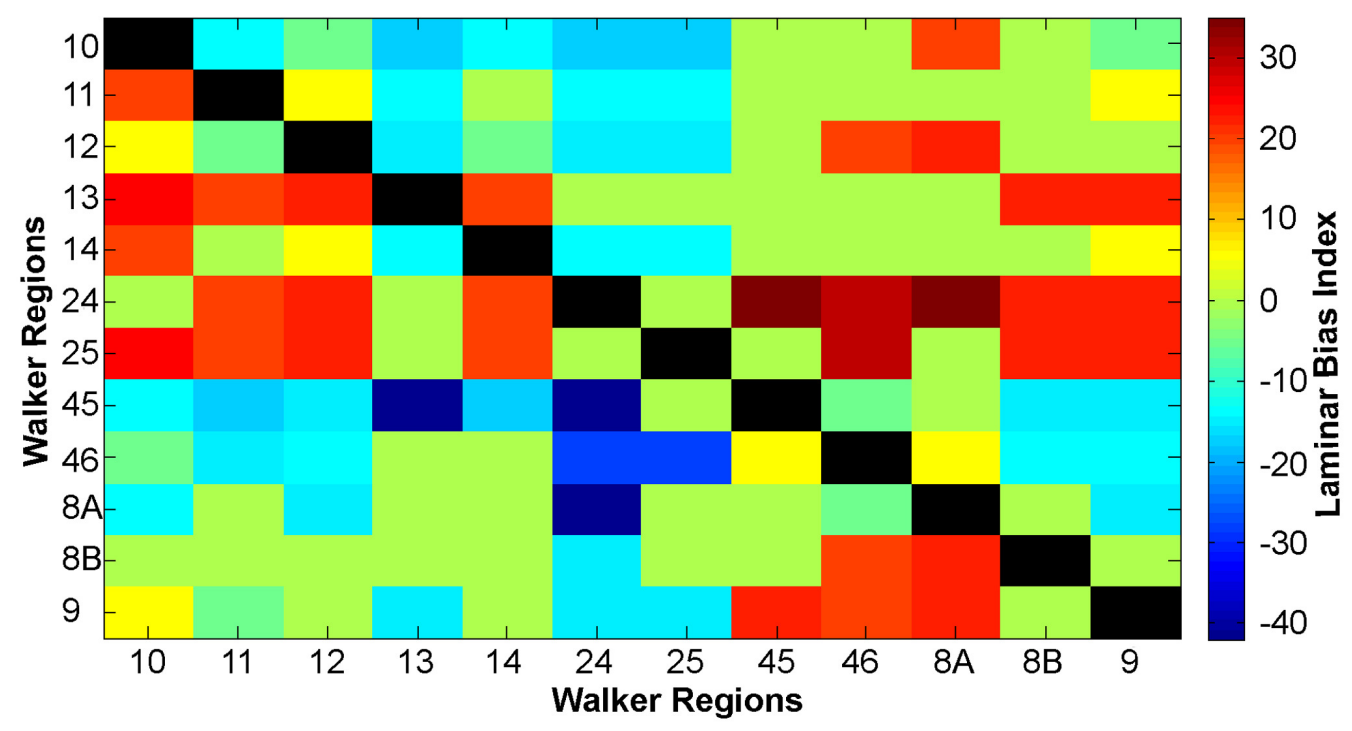

Figure S1. Matrix depicting the estimated Laminar Bias Index between the regions of the Walker's parcellation scheme. These values were subsequently used for the LBH optimization. Intraregional connections are not taken into account and therefore are not assigned a value (black boxes). 
Chapter 4

\section{Quantitative comparative analysis of structural connectivity patterns in the macaque and human brain}

Goulas $A^{*}$, Bastiani $\mathrm{M}^{*}$, Bezgin $\mathrm{G}$, Uylings HBM, Roebroeck A, Stiers P (in preparation) Quantitative comparative analysis of structural connectivity patterns in the macaque and human brain.

* Shared first authors 


\begin{abstract}
The brain consists of distinct interconnected regions and can be conceived as a complex network. Connectivity constraints function and hence large scale connectivity can be a guide to cognition. Due to methodological and ethical constraints, our most detailed knowledge about the brain stems from animal research. Specifically, the macaque brain functions as a model for the human brain, but its suitability is challenged by unique human features that emerged during primate evolution. Here we perform a quantitative comparative analysis of the whole brain connectivity of the two species. Our findings suggest that the human and macaque brain as a whole are similarly wired, while a region-wise analysis also indicates discrepancies between the species. We unravel a common structural backbone in both species involving a highly overlapping set of regions. This structure, important for mediating information across the brain, appears to be a feature of the primate brain persevering evolution. While our study argues for the suitability of the macaque brain as a model for the human brain, some discrepancies seem to exist and constrain certain macaque-human extrapolations. Our findings illustrate novel evolutionary aspects of the connectional architecture of the primate brain and offer a translational bridge between macaque and human research.
\end{abstract}




\section{$\underline{\text { Introduction }}$}

Over a century of research has revealed that the brain is inhomogeneous and can be parcellated based on topological, macro- and micro- structural criteria (Campbell, 1905; Brodmann, 1909; Walker, 1940; Tzourio-Mazoyer et al., 2002; Kötter and Wanke, 2005). The regions resulting from such a parcellation are linked through fibre bundles that constitute the neural substrate for the exchange of information between the regions (Hagmann et al., 2008). Early investigators linked these fibre bundles to the function associated with a region, thus establishing the ground of structure-function dependencies and highlighting the importance of brain connectivity for fundamental and clinical research (Campbell, 1905; Ffytche and Catani, 2005). In recent years, computational and empirical studies offered a quantitative basis for the relation of structural connectivity and function in primate and rodent brains, suggesting that the connectivity of a brain region constraints its function (Kötter and Sommer, 2000; Burns and Young, 2000; Passingham et al., 2002). Hence, regions with similar connectivity might be involved in similar functions, and large scale connectivity can be used as a guide to cognition (Bressler and Menon, 2010).

Due to ethical and methodological limitations our most detailed knowledge of the brain originates from animal research. Specifically, the macaque brain serves as a model for the human brain, but such extrapolations might be inaccurate due to rewiring and/or expansion of the brain during primate evolution (Semendeferi et al., 2002; Krubitzer and Kaas, 2005; Van Essen and Dierker, 2007) masking out unique features of the human brain (Preuss, 2000). This has important consequences for translating macaque research to humans, which is valuable for cognitive, systems and clinical neuroscience. Hence, when possible, there is the need for direct comparative examination of properties of the brain of the two species, such as their connectivity (Passingham, 2009).

Advancements like diffusion weighted magnetic resonance imaging (dwMRI) pave the way for the in vivo tracing of the structural connections between the different regions of the brain. This allows the comparison of structural connections of the human brain with those of the macaque unravelling similarities, but also differences, between the two species (Frey et al., 2008; Croxson et al., 2005; Thiebaut de Schotten et al., 2012; Petrides et al., 2012). However, such studies up to date focus on a subset of brain regions, examining particular fasciculi, or lack direct quantitative comparisons of the connections between the two species. Hence, interspecies structural connectivity differences and similarities at the global whole brain level remain concealed.

Recently techniques like dwMRI are used for the examination of the whole brain structural connectivity of the human brain, i.e. the human connectome (Sporns et al., 2005). Connectome analysis treats the brain as a complex network and employs tools from network science in order to unravel key properties that are pivotal for its proper function. Such properties include the identification in the human brain of regions that are topologically central and thus important for information integration (Hagmann et al., 2008), the so called small-world property allowing a combination of segregation and integration of information (Achard et al., 2006), and the presence of a tightly interwoven structural core forming the so called rich-club (van den Heuvel and Sporns, 2011). The usefulness of such approaches is also reflected by the novel insights obtained from studies examining the human connectome during development (Supekar et al., 2009) and clinical populations (van den Heuvel et al., 2010). Additionally, a recent network analysis unravels key properties of the macaque brain connectivity (Modha and Singh, 2010) offering hints about differences and similarities between the 
"connectome properties" of the two species, but without direct comparisons taking place.

In the current study, in order to extend and surpass limitations of previous comparative studies, we perform a direct comparative quantitative analysis of the structural connections of the human, as assessed with dwMRI, and macaque brain, as revealed by a meta-analysis of tracing studies which constitute the gold standard for estimating connectivity in the brain. By adopting a whole brain approach, we construct species-specific connectomes and quantify the similarity of connectivity patterns, global topological features, and embedding of brain regions in order to gain insight in their possible perseverance or change during primate evolution.

\section{Materials and Methods}

Whole brain parcellation scheme

For the whole brain examination of both species we employed a map specifically designed for this purpose, the Regional Map (RM) (Kötter and Wanke, 2005; Bezgin et al., 2012). This map consists of assumed homologues between the two species based on structural, topological and functional criteria. Its level of coarseness is dictated from the size of regions that are generally accepted to be discernible and homologous in both human and macaque brains (Kötter and Wanke, 2005). No connectivity criteria were used for the delineation of the various regions constituting the RM. The RM was delineated on the F99 standard brain which is based on an MRI scan of one macaque brain. Subsequently, the RM was deformed to match the human brain, in MNI space, by using macroscopic and functional landmarks (Van Essen, 2004; Orban et al., 2004). In total 82 regions (41 for each hemisphere) constituted the RM that we used (Supp Table $1)$.

\section{Macaque whole brain connectome}

We used the RM and the CoCoMac database (http://cocomac.g-node.org) to assemble the whole brain connectome of the macaque. Briefly, the CoCoMac database consists of entries describing the presence or absence of a structural connection between two regions, as revealed by tracing studies, and have the format: region $\mathrm{A}$ has an efferent connection with region $\mathrm{B}$. The database contains over 400 studies spanning several decades and thus represents our current best estimate of the connectivity of the macaque brain. Different researchers use different maps with divergent nomenclature. In order to link the different maps the database contains relation codes with the format: Region A of map $\mathrm{X}$ is identical with/smaller, larger than/partially overlapping with region B of map Y. Dedicated algorithms and algebra is used to map regions of one map to regions of a "reference" map (Stephan et al. 2000; Bezgin et al., 2008). In the current study, the $\mathrm{RM}$ functioned as the "reference" map and thus available connectivity information contained in the database was represented as an $\mathrm{NxN}$ connectivity matrix, where $\mathrm{N}=82$ the regions constituting the RM. The matrix entry Aij denotes the presence or absence of a connection from region $\mathrm{i}$ and $\mathrm{j}$ (connection $0=$ absent, $1=$ weak, $2=$ medium, 3 =strong) and constitutes the whole brain connectome of the macaque brain (for more details see Bezgin et al., 2012). Entries in the connectivity matrix with unknown status were treated as absent. In order to compare the connectivity of the human and macaque brain (see below), and since directionality of structural connections cannot be inferred 
in vivo with dwMRI in the human brain, the directed connectivity matrix of the macaque was symmetrized and binarized by taking into account all connections irrespective of their strength. Thus, the macaque connectome (MC) consisted of 1857 undirected connections/edges between 82 regions/nodes, resulting in a density of 0.559 . Density expresses the number of existing connections divided by the number of all possible connections in a connectivity matrix with $\mathrm{N}$ nodes. It ranges from 0 (no connections present) to 1 (all possible connections present).

Human whole brain connectome

Data acquisition Whole brain scans of five healthy subjects ( 2 females, age mean: 29.4 std: 3.2) were acquired after obtaining written informed consent. The experiment was approved by the local ethical committee. Data acquisition and preprocessing are described in Bastiani et al. 2012.

Voxel-wise diffusion model Multiple possibly non-orthogonal orientations of neuronal fibers were estimated per voxel. Fiber orientation distributions (FODs) were reconstructed by constrained spherical deconvolution (Tournier et al., 2007) over a fivefold tessellated icosahedron. This technique was selected for its robustness in estimating orientational distributions from high angular resolution diffusion imaging data. Moreover, FODs represent actual fiber orientation distributions rather than water-bound spin displacements, which leads to stable and accurate local orientations that are very beneficial for both local and global tractography purposes (Fillard et al., 2011).

Tractography algorithm and parameters Probabilistic local multi-direction tractography was performed using the MRtrix package (Tournier et al., 2007). The employed algorithm uses orientations sampled from the FODs at each step and initializes a great number of streamlines per seed point. Per seed point, 3000 streamlines were initiated within a sphere whose center corresponds to the center of every white matter (WM) boundary voxel and whose radius has been set to half the voxel size $(1.25 \mathrm{~mm})$. The step size was set to $1 \mathrm{~mm}$ and the angular thresholds to $30^{\circ}$. Fractional anisotropy (FA) maps were thresholded at a value of 0.1 in order to obtain the WM waypoint masks. These are binary masks containing only those voxels where fibers are allowed to propagate. The tractography algorithm used in the present study was run in original diffusion data space. Therefore, we have chosen to use median filtered FA masks computed in that same space, instead of white matter masks obtained from T1-weighted volumes segmentation, in order to achieve maximum integrity and alignment of WM masks to the diffusion data. To avoid influences on FA such as partial volume effects at the WM and grey matter boundary and in those voxels where more than two diffusion directions are reconstructed a 3-dimensional median filter was applied to the thresholded WM volumes to fill holes in the masks. Furthermore, in the WM binary masks, WM boundary voxels were always included in the volume after having thresholded the FA mask and used the median filter. Fibers shorter than $10 \mathrm{~mm}$ or longer than $200 \mathrm{~mm}$ were removed. Moreover, looping fibers, i.e. fibers that return to already explored voxels, were excluded from the analysis. To move from a very high resolution tractography result that connects all $\sim 30000$ voxels in the WM boundary voxel set to the weighted connectivity matrix based on the 82 regions of the RM, we employed the connectivity index defined in (Iturria-Medina et al. 2011). This index of connectivity between two regions is given by the sum of the weights connecting all the 


\section{Chapter 4}

voxels between region $\mathrm{A}$ and region $\mathrm{B}$ and vice versa, normalized by the sum of the number of seeds used in each region. Thus, any non-zero weight connecting any voxel in one parcel to any voxel in another in either of the two directions connects the two parcels in the final symmetric adjacency matrix (Iturria-Medina et al., 2011). Such step reconstructs the weighted $82 \times 82$ connectivity matrix by eliminating the effect of patcharea size. To create the human connectome (HC) the symmetrized weighted matrices obtained from each individual were averaged. Subsequently, in order to render the MC and $\mathrm{HC}$ comparable, only the strongest weights were selected and their number was chosen to match the number of connections of the MC. Finally, the HC was binarized. Hence, the $\mathrm{MC}$ and $\mathrm{HC}$ are binary undirected matrices with the same number of nodes and connections.

Comparison of macaque and human connectomes

Interspecies whole brain connectivity similarity The first question to be addressed is maybe the most general and simple one: Are the connectomes of the two species significantly similar in a statistical sense? To this end, we calculated the edit distance (ED) between the matrices derived from the two species. Given two matrices, the ED is defined as the number of insert/delete operations to be performed for the conversion of one matrix to the other (Trusina et al., 2005). More formally the ED between matrices A and $\mathrm{B}$ is defined as:

$$
E D(A, B)=\sum_{i, j}\left|A_{i j}-B_{i j}\right|
$$

Hence, a small (large) ED indicates high (low) similarity of A and B, where A represents the MC and B the HC. Similar techniques have been used for the assessment of the functional similarity of regulatory networks (Trusina et al., 2005). We should note that the ED was normalized in order to range from 0 to 1 by dividing with the maximum possible ED given the matrices at hand. For the sake of simplicity we shall refer to the normalized values simply as ED.

Interspecies region-wise connectivity similarity As a next step, we focused on a more detailed region-wise analysis. The RM consists of assumed homologues based on structural, topological and functional criteria. The aforementioned criteria are commonly used for the establishment of homologies between two species. Additionally, the similarity of connectivity patterns also constitutes a criterion for homologies (e.g. Campbell and Hodos, 1970; Uylings and Van Eden, 1990). Hence, our analysis aimed at establishing if the assumed homologue regions, as dictated by the RM, also exhibit a statistically significant connectivity similarity. To this aim, we calculated the overlap of the connections of the assumed homologues of the macaque and the human brain as dictated by their connectomes. More specifically, we calculated the homologue connectivity similarity (HCS):

$$
\operatorname{HCS}_{i}(A, B)=\frac{A_{i} \bigcap B_{i}}{A_{i} \bigcup B_{i}}
$$


The interspecies overlap/intersection of connections of region $\mathrm{i}$ in the macaque and human brain, represented by $\mathrm{A}$ and $\mathrm{B}$ respectively, is denoted by $A_{i} \cap B_{i}$ and the union with $A_{i} \cup B_{i}$. Hence, for each region $\mathrm{i}=1 \ldots 82$ of the $\mathrm{RM}$ the $\mathrm{HCS}_{\mathrm{i}}$ ranges from 0 to 1 and indicates respectively low and high interspecies connectivity similarity of the assumed homologue region i. The HCS resembles the matching index (Hilgetag et al., 2002) with the only difference that it is calculated between nodes of different connectivity matrices, i.e. A and B representing the $\mathrm{MC}$ and $\mathrm{HC}$ (see Fig. $1 \mathrm{~B}$ ).

A

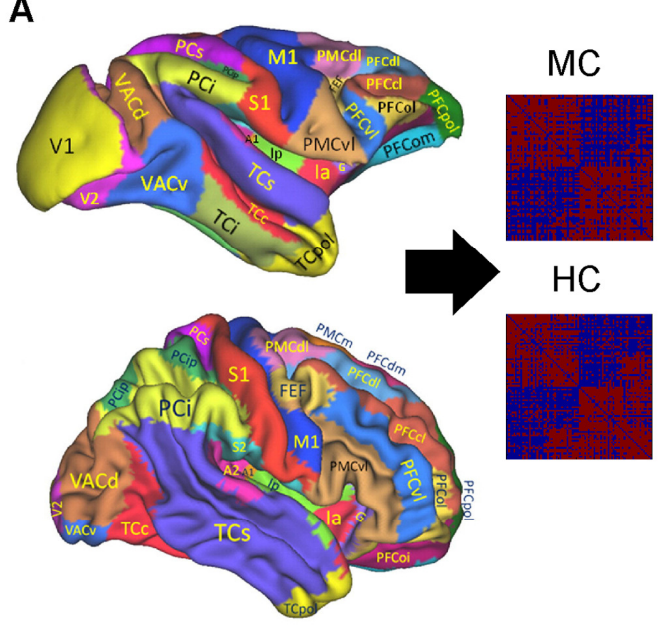

C

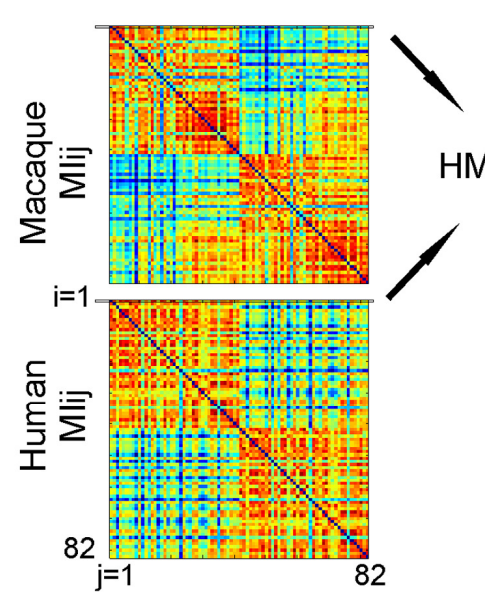

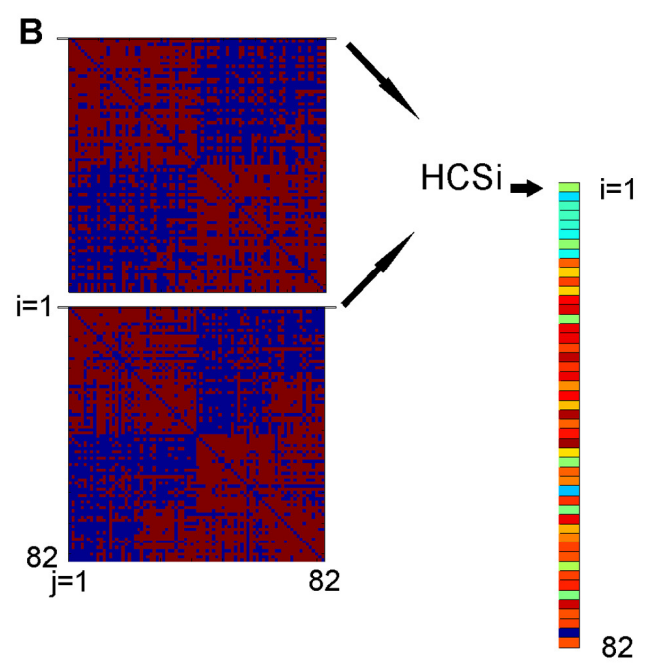

D

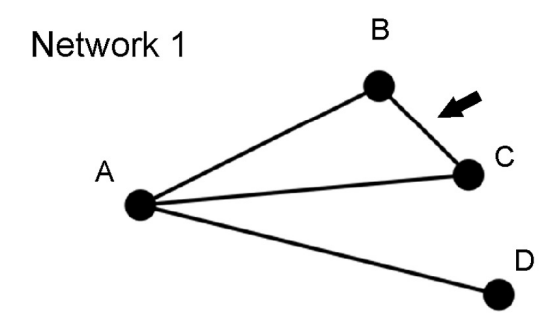

Network 2

B

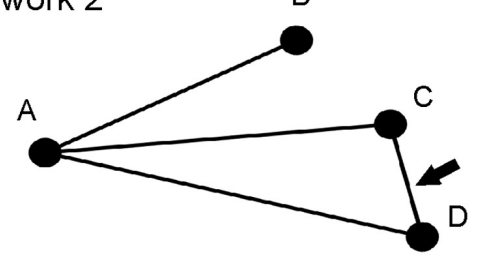

Figure 1. Schematic depiction of the main points of the analysis. A. The RM was used in order to construct the connectivity matrices of the two species, i.e. the $\mathrm{MC}$ and $\mathrm{HC}$. The resulting connectivity matrices have $\mathrm{i}=82$ rows and $j=82$ columns. B. Example of the calculation of the HCS for region $i=1$. To this end the entries of row 1 from the $\mathrm{MC}$ and row 1 from $\mathrm{HC}$ were used, resulting in entry $\mathrm{HCS}_{\mathrm{i}=1}$. The same procedure was used for all regions, resulting in a 1x82 vector HCS containing the HCS indexes of all the regions. C. Example of calculation of the HMIS for region $\mathrm{i}=1$. The $\mathrm{MC}$ and $\mathrm{HC}$ were used for the separate calculation of the matching index matrices $\mathrm{MI}_{\mathrm{ij}}$ (one for each species separately). The HMIS index for region $\mathrm{i}=1$ is calculated from the entries of row 1 of the matching index matrices of the two species. The procedure is repeated for all regions, resulting in a 1x82 vector HMIS containing the HMIS indexes of all the regions. D. Toy networks demonstrating potential differences of the HCS and the HMIS for a region. Given two hypothetical "brains" that form a network with 4 regions and 4 connections the HCS and HMIS indexes are calculated for brain region A. On the one hand, the HCS index is equal to 1 (perfect similarity), since region A in both networks 


\section{Chapter 4}

that could be the brains of two species, is connected exactly to the same regions, i.e. nodes B, C and D. On the other hand, the HMIS index is -0.5 , indicating a divergence of the connectivity similarity profiles of region A in networks/brains 1 and 2. These discrepancies arise from the "rewiring" of the connection marked with an arrow. While in network 1 region A shares neighbours with node B in network 2 no such sharing exists, leading to a change in the connectivity similarity profiles of region A.

Interspecies region-wise matching index similarity The next step of the analysis examined the similarity of the connectivity patterns of homologous regions within the brain of each species. Conceptual arguments and empirical evidence suggest that the connectivity of a region with the rest of the brain constraints its function (Passingham et al., 2002; Passingham and Wise, 2012). Consequently, similarity of connectivity between two regions can entail functional similarity. For instance, when taking into account the connectivity of the cat cortex four different modules are discernible. Such modules correspond to distinct anatomical structures that exhibit relative functional specializations (e.g. visual vs somatosensory). Regions belonging to the same module are characterized by higher similarity of their structural connections with the rest of the brain when compared to regions belonging to different modules (Zamora- López et al., 2010). Hence, we examined if the within species whole brain connectivity similarity of each region in each species was preserved for homologous regions. To this end, we used the matching index (Hilgetag et al., 2002) for a matrix X (i.e. either the MC or the HC):

$$
M I_{i j}=\frac{X_{i} \bigcap X_{j}}{X_{i} \bigcup X_{j}}
$$

The result is the matching index matrix MI whose entry MIij for a given matrix $\mathrm{X}$ quantifies the similarity of connections of region/node $i$ and $j$, not taking into account potential connections between $i$ and $j$, with values ranging from 0 (no similarity) to 1 (identical). Hence, each row $\mathrm{MI}_{\mathrm{i}}$ describes the connectivity similarity of region i with the rest of the brain regions and can be considered as the connectivity similarity profile of region i. It should be noted that the MI was calculated separately for each connectivity matrix $\mathrm{A}$ and $\mathrm{B}$ (representing the $\mathrm{MC}$ and $\mathrm{HC}$ respectively). This resulted in two MI matrices $\mathrm{MI}_{\mathrm{A}}$ and $\mathrm{MI}_{\mathrm{B}}$ for each species. In order to quantify if the connectivity similarity profile of homologous regions was preserved, we calculated the correlation between row $\mathrm{i}=1 \ldots 82$ of matrices $\mathrm{MI}_{\mathrm{A}}$ and $\mathrm{MI}_{\mathrm{B}}$. This resulted in the homologue matching index similarity (HMIS):

$$
H M I S_{i}\left(M I_{A}, M I_{B}\right)=r\left(M I_{A i}, M I_{B i}\right)
$$

with $r$ denoting Pearson's correlation coefficient of row $i$ of $\mathrm{MI}_{\mathrm{A}}$ and $\mathrm{MI}_{\mathrm{B}}$ (Fig. $1 \mathrm{C}$ ). Hence, HMIS ranges from -1 to 1 with high values indicating that the connectivity similarity of region $i$ with the rest of the regions of the macaque brain resembles the connectivity similarity of region $i$ with the rest of the regions of the human brain, thus forming the same "connectivity coalitions" with the rest of the brain in both species. Low and increasingly negative values of HMIS indicate low resemblance of the connectivity similarity of region $i$ with the rest of the brain of each species, suggesting changes of "connectivity coalitions" of region $\mathrm{i}$.

Centrality and clustering of the macaque and human connectomes This step of the analysis aimed at associating certain key network properties of the $\mathrm{MC}$ and $\mathrm{HC}$. One of 
the important properties of the primate brain that network analysis has revealed is the presence of a subset of regions that through the way they are embedded in the structural whole brain network play an important role in integrating and mediating information that flow across the brain. These regions are thus topologically central and are known as "hubs" (Sporns et al., 2007). The importance of such regions is reflected in their high metabolic costs, their depletion in brain pathologies such as Alzheimer's disease and the pronounced global effects accompanying their lesions (Sporns et al., 2007; Buckner et al., 2009; Hagmann et al., 2008). Additionally, network analysis has revealed that the brain also exhibits non-random features like high levels of clustering/segregation, i.e. certain groups of regions are tightly interconnected. Such a feature indicates the presence of a structure supporting specialized information processing (Rubinov and Sporns, 2010).

We aimed at examining to what extent the centrality and segregation of homologous regions was preserved across the species. To this end, we calculated betweenness centrality (BC) (Freeman, 1977), eigenvector centrality (EC) (Bonacich, 1972) and clustering coefficient (CC) (Watts and Strogatz, 1998) for all the regions of the brain of each species separately. Briefly, BC of node i quantifies the amount of the shortest paths that pass through node $\mathrm{i}$ divided by the total number of such possible paths. Hence, regions with high $\mathrm{BC}$ are central way stations of network traffic. The EC of node $\mathrm{i}$ equals the ith entry of the principal eigenvector of the connectivity matrix. The EC of a node is high when the node exhibits many connections, i.e. has high degree, but favours nodes with neighbours that in turn exhibit high EC. Hence, regions with high EC are ideally embedded for integrating information across the brain. The $\mathrm{CC}$ quantifies the connections existing between the neighbours of a region divided by the total possible number of such connections. All the above metrics (BC, EC and CC) were computed by using the formulas for binary undirected networks as specified in (Rubinov and Sporns, 2010; Zuo et al., 2012).

The above metrics are calculated for each region and species separately. Thus each metric results in one $1 \times 82$ vector for each species describing the metric of each of the 82 regions of the RM. In order to assess preservation of the centrality and clustering of the brain of the two species as a whole, the Pearson's correlation coefficient between the two vectors from the two species describing the same metric was computed. A statistically significant (positive) correlation would indicate preservation of the metric across the brain of the two species.

The aforementioned analysis is important in order to unravel topological similarities and differences of the embedding of the brain regions during primate evolution. This in turn can provide heuristics for the usefulness and validity of extrapolation of findings from network analysis in the macaque to the human brain. For instance, such extrapolations can offer indications about possible effects of lesions in the human brain (Alstott et al., 2009) and potential key regions that are related to neurological diseases (van den Heuvel et al., 2010).

Rich clubs in the macaque and human connectomes Recent network analysis of the human and macaque connectome uncovered a tightly interwoven structural core forming the so called rich club (van den Heuvel and Sporns, 2011; Harriger et al., 2012). The presence of a rich club organization is quantified with the rich club coefficient (RCC) (Colizza et al., 2006): 
Chapter 4

$$
\varphi(k)=\frac{2 E_{>k}}{N_{>k}\left(N_{>k}-1\right)}
$$

where $\mathrm{E}_{>\mathrm{k}}$ denotes the number of connections/edges that exist among nodes/regions that exhibit more connections than a given number $\mathrm{k}$ and $\mathrm{N}_{>\mathrm{k}}$ denotes the number of nodes/regions that have degree higher than $\mathrm{k}$, i.e. exhibit more connections than a given number $\mathrm{k}$. The RCC is calculated for a range of $\mathrm{k}$ for a given network and for a number of randomized matched networks in order to estimate the RCC values expected by chance (see Statistical assessment). This results in a normalized RCC:

$$
\varphi_{\text {norm }}(k)=\frac{\varphi(k)}{\varphi_{\text {rand }}(k)}
$$

Consequently, values higher than 1 for a range of values $\mathrm{k}$ indicate that the network is characterized by a rich club structure, with nodes with degree higher than $\mathrm{k}$ linked with more connections expected by chance (Colizza et al., 2006).

This structure functions as a structural backbone mediating the flow of information at the whole brain level and hypothesized to give rise to a "greedy degree-based" routing of information in the brain (van den Heuvel et al., 2012). Moreover, the importance of the rich club structure is reflected in the pronounced deterioration of the efficiency of the $\mathrm{HC}$ after targeted attacks to regions of the rich club. Hence, neurological diseases, strokes or brain injuries affecting such regions might have more widespread effects and disturb neurocognitive functions at multiple levels (van den Heuvel and Sporns, 2011). Additionally, network analysis of the connectome of carnivores, i.e. cat, has revealed the presence and pivotal role of the rich club structure in multisensory integration (Zamora- López et al., 2010).

Prompted by the aforementioned findings illustrating the importance of the rich club structure, we aimed at tracing the rich club structure in the $\mathrm{HC}$ and MC. Detecting a rich club in the $\mathrm{MC}$ and $\mathrm{HC}$ is of secondary importance since recent studies have demonstated its presence. What is of primary interest in the current study is how the rich clubs in the $\mathrm{HC}$ and $\mathrm{MC}$ are related. Analysis conducted separately in the MC and $\mathrm{HC}$ indicates that certain rich club regions are topologically similar in the two species (van den Heuvel and Sporns, 2011; Harriger et al., 2012). However, different parcellation schemes were employed for the analysis of the HC and MC. Such parcellation schemes are not directly comparable since they exhibit different levels of coarseness and are based on either topological criteria or arbitrary parcels, with respect to the $\mathrm{HC}$, and diverse and not always consistent parcellations, mainly based on microstructural criteria, with respect to the MC. Hence, despite evidence that certain rich club regions in the two species are similar, from a topological point of view, such results can not be directly compared. The current approach, by the adoption of the RM that offers a common parcellation scheme adequate for comparative purposes, renders a direct comparison of the rich club in the two species possible.

The above comparative examination of the rich club of the $\mathrm{MC}$ and $\mathrm{HC}$ is of crucial importance in gaining insights into the evolutionary course of the structural backbone of the primate brain. Furthermore, it also provides a translational bridge between macaque and human brain research by offering heuristics of potential deficits and properties of 
brain regions constituting the rich club, e.g. predicting impact of brain lesions/injuries of humans from lesions performed in macaques.

\section{Statistical assessment}

For assessing the statistical significance of the aforementioned analyses we performed each analysis on 10000 random networks matching each $\mathrm{MC}$ and $\mathrm{HC}$ in number of nodes, edges and degree distribution. The degree preserving rewiring algorithm was used for the generation of the random networks (Maslov and Sneppen, 2002). For example, in order to assess the significance of the HCS obtained from the original MC and $\mathrm{HC}$, the HCS was calculated by using random instances of the MC and HC. This results in $10000 \mathrm{HCS}$ indexes indicating for each RM region its corresponding null HCS value. In this way p-values and z-scores can be computed. The z-scores of each metric of interest thus offer a normalized estimate of each metric. The p-values are used for assigning statistical significance to the results and for the region-wise analysis, introducing multiple tests, we used a conservative Bonferroni correction. Similar techniques have been used for the statistical assessment of topological features in the HC (e.g. van den Heuvel and Sporns, 2011).

\section{Control analyses}

In order to verify the robustness of the findings the following procedure was followed. The MC and $\mathrm{HC}$ were perturbed by rewired their connections with probability 0.1 and thus a scrambling of their topology took place. Subsequently, we calculated the ED, HCS, HMIS and RCC of the scrambled MC and $\mathrm{HC}$ and assessed their significance. If this random scrambling does not have a detrimental impact on the results, e.g. a potential rich club structure in the original networks is still present and significant, then we can conclude that the findings are robust. Similar techniques have been used for assessing the robustness of properties of the HC (Hagmann et al., 2008) and the modular structure of the lateral frontal cortex (Goulas et al., 2012).

The CoCoMac database used for assembling the MC, despite that it contains results from numerous studies, is not complete. Moreover, recent tracing studies in the macaque brain indicate the presence of connections never previously reported (Markov et al., 2011). Thus, the following simple control analysis was conducted. We inserted $10 \%$ of the connections of the MC $(1857 * 10 \%=186$ connections $)$ in empty entries of the connectivity matrix describing the $\mathrm{MC}$ at random (excluding the diagonal). Subsequently, the weighted matrix obtained from the human subjects was thresholded as described before but now the threshold was chosen in such a way that the resulting $\mathrm{HC}$ matched the new number of MC connections. All the analysis was conducted again with the new "randomly enriched" MC and the corresponding HC. If the results obtained are still significant we can assume that the presence of "new connections" does not have a profound impact on the initial results. Despite that this control analysis involves the random unstructured insertion of connections, such analysis constitutes a simple "simulation" of the scenario that certain connections denoted as absent might appear to be present.

The construction of the MC and $\mathrm{HC}$ relied on the RM. In order to examine if the choice of this particular parcellation has an effect on our results, we performed all the described analysis on the connectomes assembled based on the parcellation scheme of Von Bonin and Bailey, 1947 (BB47). The way of assembling the MC and $\mathrm{HC}$ was 
performed as described before for the RM, but now the BB47 parcellation scheme functioned as a "reference" map.

\section{$\underline{\text { Results }}$}

Interspecies whole brain connectivity similarity The ED between the MC and $\mathrm{HC}$ was significantly lower than the one obtained between matched random networks (EDoriginal $=0.303 \mathrm{p}<0.0001$, EDnull mean $=0.494 \mathrm{std}=0.007$ ). Thus, the wiring of the macaque and human brain as a whole is more similar than expected by chance.

Interspecies region-wise connectivity similarity The region-wise analysis of the HCS revealed that many structural, topological and functional homologues, as entailed by the RM, also exhibit significant preservation of their connections (Table 1, Fig. 2 A). More specifically, a set of frontal, certain limbic, occipital and temporal regions exhibited significant preservation of their whole brain connectivity across the species. Only a set of regions, mainly parietal and limbic, appeared to lack such preservation (Fig. 2 A). In total, 51 out of 82 RM regions exhibited a significant HCS $(p<0.05$ Bonferroni corrected). Hence, more than half of RM regions showed preservation of connectivity during primate evolution and therefore, in both species, communicate with a significantly overlapping set of brain regions.

Interspecies region-wise matching index similarity The region-wise analysis of the HMIS resulted in a significant HMIS for 45 out of 82 RM regions, mainly involving frontal, temporal, occipital regions (Table 1, Fig. 2 B). 37 out of 82 RM regions exhibited HMIS values below significance $(\mathrm{p}<0.05$ Bonferroni corrected), involving mainly the limbic and parietal regions. Hence, regions reaching significance seem to form the same "connectivity coalitions", i.e. exhibit a statistically significant connectivity similarity profile with the rest of the brain in both humans and macaques. This in turn can entail that their "functional coalitions" might also be preserved. Conversely, certain regions fail to reach a significant HMIS. This discrepancy might suggest that "evolutionary rewiring" occurred in such a way that they formed distinct "connectivity coalitions" with the rest of the brain regions in the two species.

It should be noted that the HCS and HMIS involve different regions exhibiting significant values but also involve a large set of overlapping regions, mainly located at the frontal, occipital and temporal cortices (Table 1, Fig. 2). The above illustrate converging but also distinct aspects of connectional characteristics of the regions of the brain of the two species. 
Table 1. HCS and HMIS values for all the RM regions.

\begin{tabular}{|c|c|c|c|c|c|c|c|c|}
\hline \multirow{3}{*}{$\begin{array}{c}\text { RM } \\
\text { acronyms }\end{array}$} & \multicolumn{4}{|c|}{ Right } & \multicolumn{4}{|c|}{ Left } \\
\hline & \multicolumn{2}{|c|}{ HCS } & \multicolumn{2}{|c|}{ HMIS } & \multicolumn{2}{|c|}{ HCS } & \multicolumn{2}{|c|}{ HMIS } \\
\hline & z-score & p-value & z-score & p-value & z-score & p-value & z-score & p-value \\
\hline Tcpol & \begin{tabular}{|l|l|}
3.18 \\
\end{tabular} & 0.0012 & 2.47 & 0.0066 & 3.66 & 0.0001 & 2.11 & 0.0166 \\
\hline TCs & 4.29 & 0.0001 & 4.13 & 0.0001 & 4.26 & 0.0001 & 4.37 & 0.0001 \\
\hline Amyg & 4.92 & 0.0001 & 4.99 & 0.0001 & 4.86 & 0.0001 & 5.60 & 0.0001 \\
\hline PFCoi & 3.08 & 0.0015 & 2.94 & 0.0014 & 3.31 & 0.0007 & 2.61 & 0.0042 \\
\hline la & 4.65 & 0.0001 & 4.47 & 0.0001 & 4.01 & 0.0001 & 4.78 & 0.0001 \\
\hline PFCom & 3.39 & 0.0003 & 3.57 & 0.0001 & 2.41 & 0.0135 & 5.08 & 0.0001 \\
\hline TCc & 5.13 & 0.0001 & 4.68 & 0.0001 & 4.45 & 0.0001 & 4.51 & 0.0001 \\
\hline PFCol & 3.77 & 0.0002 & 6.29 & 0.0001 & 3.49 & 0.0006 & 6.85 & 0.0001 \\
\hline $\mathrm{TCi}$ & 4.01 & 0.0001 & 6.14 & 0.0001 & 4.41 & 0.0001 & 5.61 & 0.0001 \\
\hline $\mathrm{PHC}$ & 3.96 & 0.0001 & 1.80 & 0.0337 & 2.74 & 0.0058 & 1.70 & 0.0432 \\
\hline G & 2.62 & 0.0046 & \begin{tabular}{|l|l}
3.77 \\
\end{tabular} & 0.0001 & 3.65 & 0.0002 & 5.45 & 0.0001 \\
\hline PMCvl & 3.82 & 0.0001 & 5.62 & 0.0001 & 4.35 & 0.0001 & 5.76 & 0.0001 \\
\hline VACv & 4.27 & 0.0001 & 5.67 & 0.0001 & 4.32 & 0.0001 & 4.92 & 0.0001 \\
\hline Ip & 1.34 & 0.1502 & 0.21 & 0.4200 & 1.18 & 0.1851 & -0.03 & 0.5145 \\
\hline PFCpol & 2.90 & 0.0040 & 3.30 & 0.0006 & 3.62 & 0.0002 & 5.15 & 0.0001 \\
\hline $\mathrm{HC}$ & 2.92 & 0.0025 & 3.64 & 0.0002 & 2.73 & 0.0033 & 3.11 & 0.0009 \\
\hline $\mathrm{CCs}$ & 2.38 & 0.0160 & 0.65 & 0.2650 & 2.53 & 0.0102 & 0.05 & 0.4836 \\
\hline PFCvl & 3.91 & 0.0001 & 4.19 & 0.0001 & 4.73 & 0.0001 & 3.79 & 0.0001 \\
\hline V2 & 3.47 & 0.0007 & 4.10 & 0.0001 & 4.09 & 0.0001 & 4.03 & 0.0001 \\
\hline PFCm & 4.35 & 0.0001 & 3.11 & 0.0007 & 3.73 & 0.0001 & 2.10 & 0.0170 \\
\hline $\mathrm{TCv}$ & 3.33 & 0.0005 & 1.94 & 0.0242 & 3.12 & 0.0010 & 1.87 & 0.0291 \\
\hline VACd & 4.32 & 0.0001 & 4.22 & 0.0001 & 3.94 & 0.0001 & 4.44 & 0.0001 \\
\hline V1 & 3.52 & 0.0001 & 3.39 & 0.0003 & 3.55 & 0.0002 & 4.48 & 0.0001 \\
\hline PFCcl & 4.86 & 0.0001 & 5.09 & 0.0001 & 4.28 & 0.0001 & 4.85 & 0.0001 \\
\hline A2 & 2.89 & 0.0039 & 2.16 & 0.0137 & 3.66 & 0.0003 & 3.34 & 0.0001 \\
\hline $\mathrm{CCr}$ & 2.14 & 0.0239 & 0.12 & 0.4633 & 2.43 & 0.0122 & -0.22 & 0.5884 \\
\hline CCp & 3.26 & 0.0009 & 1.46 & 0.0727 & 3.90 & 0.0001 & 2.09 & 0.0164 \\
\hline $\mathrm{CCa}$ & 2.27 & 0.0271 & -0.79 & 0.7836 & 1.84 & 0.0646 & -0.33 & 0.6341 \\
\hline S2 & 4.11 & 0.0001 & 3.20 & 0.0007 & 4.05 & 0.0001 & 3.07 & 0.0006 \\
\hline S1 & 3.94 & 0.0001 & 2.57 & 0.0046 & 3.33 & 0.0009 & 3.27 & 0.0004 \\
\hline A1 & 3.07 & 0.0015 & 2.58 & 0.0036 & 3.53 & 0.0006 & 3.26 & 0.0002 \\
\hline M1 & 3.67 & 0.0004 & 3.86 & 0.0001 & 3.84 & 0.0003 & 3.91 & 0.0001 \\
\hline $\mathrm{PCi}$ & 4.21 & 0.0001 & 0.01 & 0.5032 & 4.03 & 0.0001 & 0.48 & 0.3214 \\
\hline $\mathrm{PCm}$ & 2.63 & 0.0089 & 2.17 & 0.0136 & 2.91 & 0.0058 & 2.49 & 0.0052 \\
\hline PFCdm & 2.04 & 0.0360 & 1.07 & 0.1390 & 2.12 & 0.0310 & 3.36 & 0.0001 \\
\hline PCip & 3.67 & 0.0003 & 0.54 & 0.2936 & 3.24 & 0.0019 & 0.87 & 0.1910 \\
\hline PCs & 1.96 & 0.0441 & 1.90 & 0.0284 & 1.54 & 0.1036 & 1.94 & 0.0253 \\
\hline FEF & 3.37 & 0.0006 & 3.43 & 0.0001 & 2.59 & 0.0071 & 3.75 & 0.0001 \\
\hline PFCdl & 3.72 & 0.0003 & 3.82 & 0.0001 & 4.26 & 0.0001 & 3.14 & 0.0004 \\
\hline $\mathrm{PMCm}$ & 3.80 & 0.0003 & 2.57 & 0.0040 & 4.04 & 0.0001 & 2.64 & 0.0036 \\
\hline PMCdl & 4.26 & 0.0001 & 3.42 & 0.0001 & 5.30 & 0.0001 & 3.16 & 0.0007 \\
\hline
\end{tabular}

Regions exhibiting significant HCS and HMIS values ( $\mathrm{p}<0.05$ Bonferroni corrected) are in bold. The $\mathrm{p}$-values and $\mathrm{z}$-scores of the aforementioned metrics are also depicted.

Centrality and clustering of the macaque and human connectome The centrality and clustering analysis of the $\mathrm{MC}$ and $\mathrm{MC}$ revealed that, at the whole brain level, the topological embedding of the RM regions in the two species is not significantly 


\section{Chapter 4}

correlated (Supp Fig. 1, Supp Table 2). More specifically, the centrality of the regions in the $\mathrm{MC}$ and $\mathrm{HC}$, as quantified with the $\mathrm{BC}$ and $\mathrm{EC}$, exhibited a non-significant correlation (Supp Fig. 1 A B). This might suggest a lack of preservation of the topological importance, as measured by the $\mathrm{BC}$ and the $\mathrm{EC}$, of the assumed homologues of the macaque and human brain. Moreover, when taking into account only the regions that could be termed as "hubs", i.e. with a centrality measure above the mean +1 std of the totality of the RM regions, little overlap was observed between such hubs in the macaque and the human brain (Supp Table 2). This might suggest that a different set of regions in the two species function as way stations and integrators of information across the primate structural network. It should be noted however, that bilateral posterior cingulate cortex (CCp) and left inferior parietal cortex (PCi) exhibited both high $\mathrm{BC}$ and $\mathrm{EC}$ in both species, above the mean +1 std threshold, indicating the preservation of the topological role of this region as a prominent central node.

The correlation of the $\mathrm{CC}$ between the $\mathrm{MC}$ and $\mathrm{HC}$, which functions as a measure of segregation, did not reach significance either (Supp Fig. 1 C). Therefore, the regions of the brains of the two species seem to exhibit different levels of segregation/specialization (see also Supp Table 2).

Rich clubs in the macaque and human connectomes The rich club analysis revealed the presence of a rich club organization in both the MC and $\mathrm{HC}$ (Fig. $3 \mathrm{~A}$ ), in line with previous studies (van den Heuvel and Sporns, 2011; Harriger et al., 2012). Most importantly, the analysis demonstrates that the regions forming a rich club, at level $\mathrm{k}$ corresponding to the pick of the normalized RCC, exhibit a high and significant overlap $(14 / 20)$, involving the frontal and parietal cortex as well as regions of the cingulate and insular cortex (Fig. 3 B, 4, Supp Table 3). This overlap is observed for a wide range of $\mathrm{k}$ values within the rich club regime (Fig. 4). This indicates that not only the human and macaque brain exhibits a rich club organization, but that this structure constitutes a preserved structural backbone in the primate brain involving a highly overlapping set of regions in both species. 


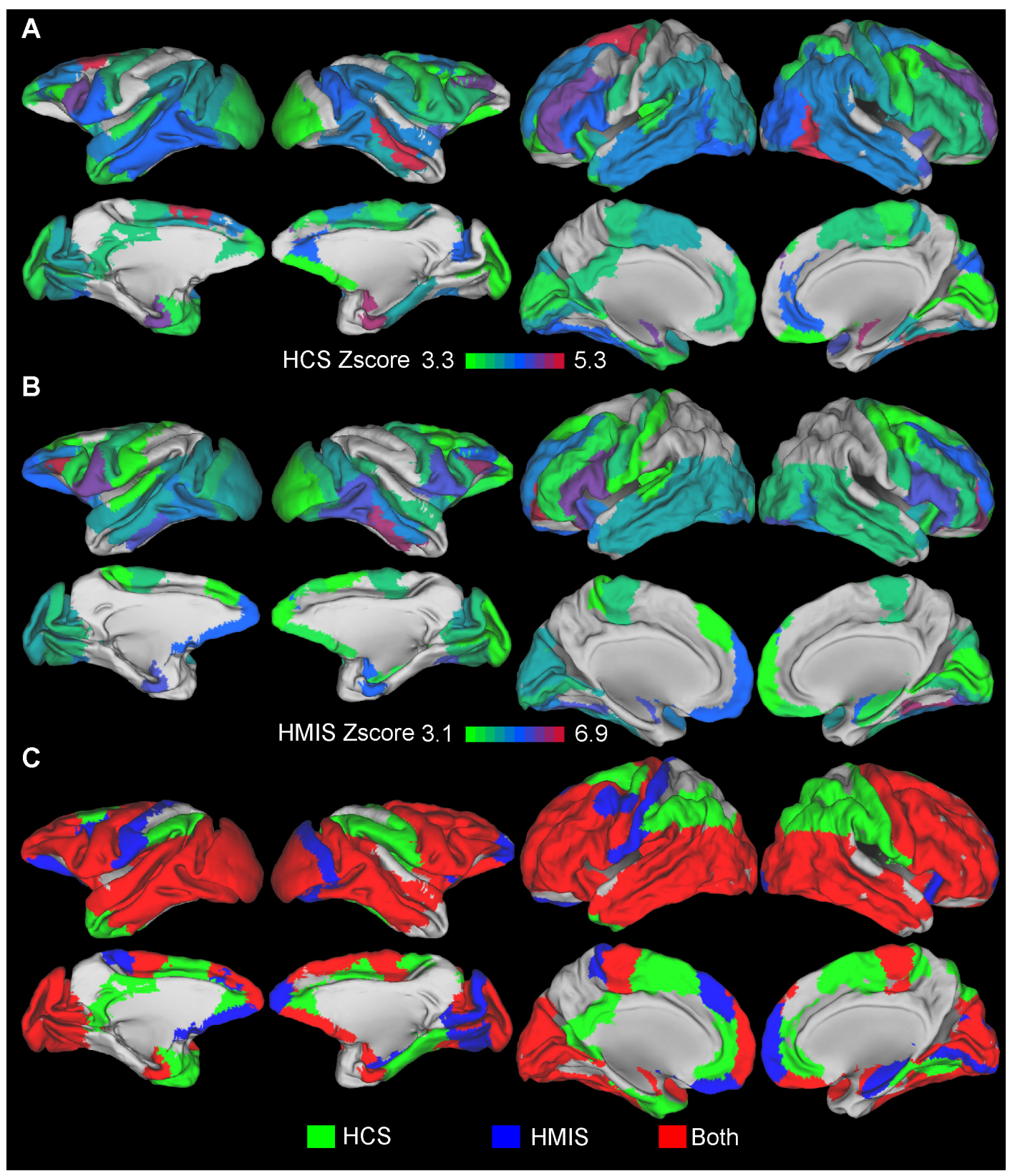

Figure 2. Renderings of the macaque and human regions exhibiting a significant A. HCS and B. HMIS index. In both panels only regions reaching a significance level of 0.05 (Bonferroni corrected) are depicted. Colour coding denotes their corresponding z-score. C. Summary of results by colour coding the regions based on the preservation of both HMIS and HCS (red), only HCS (green), only HMIS (blue). 


\section{Chapter 4}

A

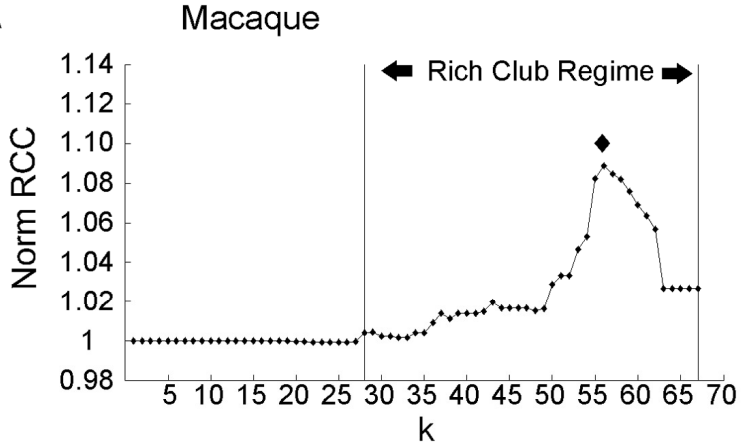

B
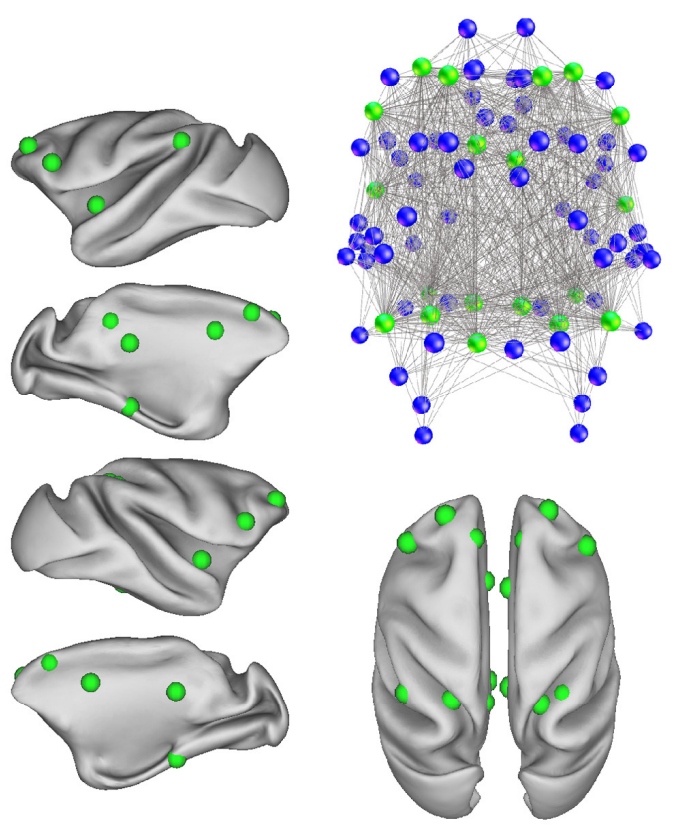

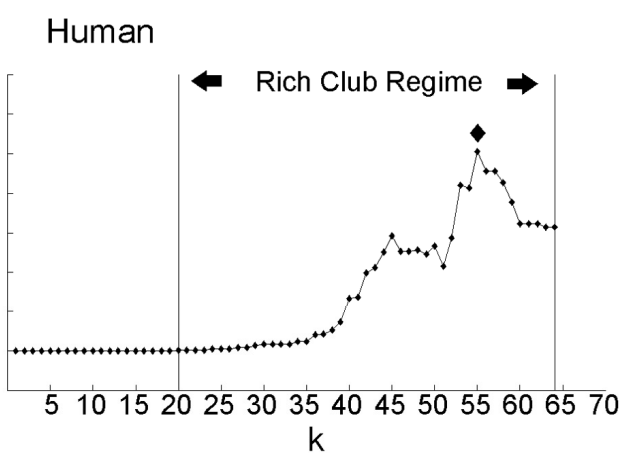

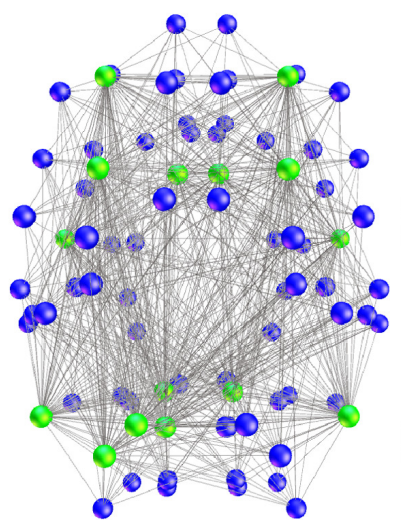
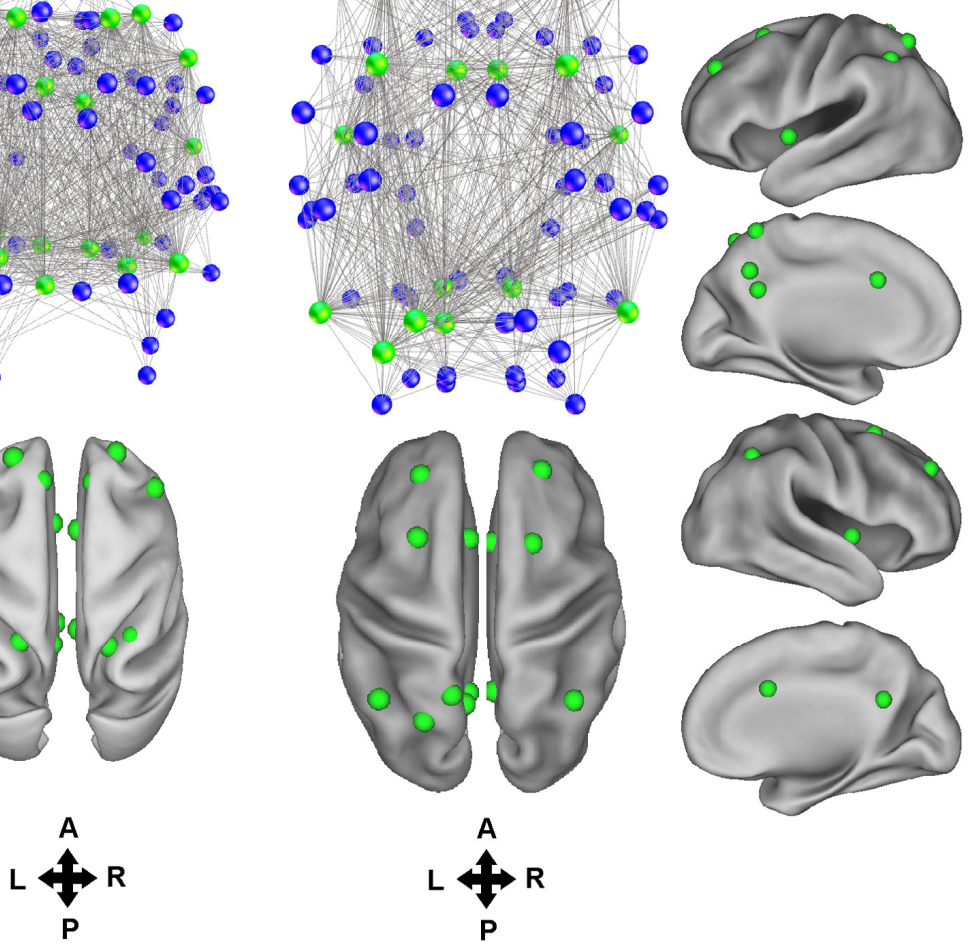

Figure 3. Rich club structure in the macaque and human brain. A. The normalized RCC suggests the presence of a rich club organization in both the human and macaque brains. The normalized RCC obtained within the rich club regime $(>1)$ were significantly higher than the ones obtained from random netwroks matched for node, edge and degree distribution $(\mathrm{p}<0.0001)$. B. Network and anatomical representation of the regions corresponding to the pick of the normalized RCC which is marked with a diamond in panel A $(\mathrm{k}=56$ for macaque and $\mathrm{k}=55$ for human). In the network representation, green and blue nodes represent rich club and non rich club regions at level k respectively. Only connections involving at least one rich club region are depicted. The anatomical representation depicts the regions constituting the rich club on the inflated fiducials of both hemispheres of the brains of the two species. The spheres represent the centre of mass of the involved regions. Note the convergence of the rich club analysis to a highly overlapping set of regions in the two species. The observed overlap is significant (see Fig. 4). 


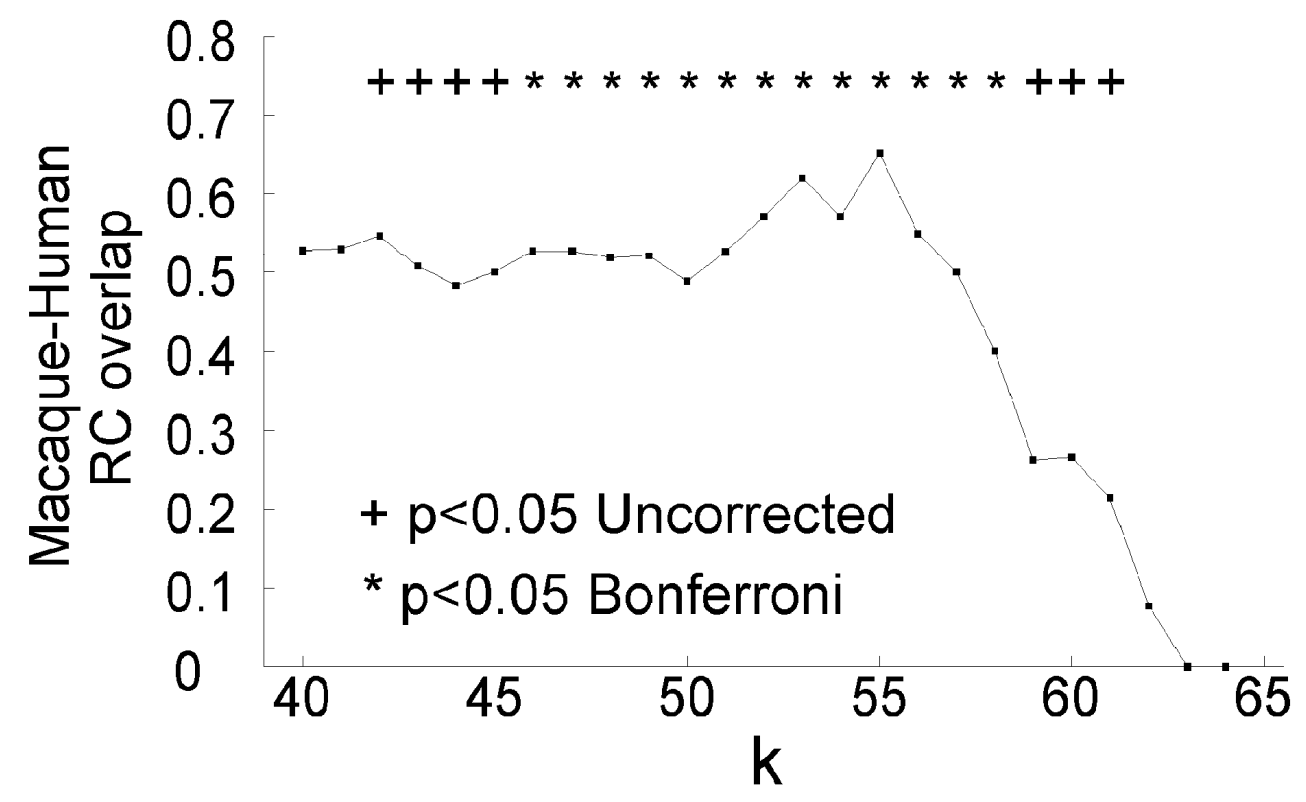

Figure 4. Overlap of the regions constituting the rich club in the two species over a range of $\mathrm{k}$ values. At each level $\mathrm{k}$, and given $\mathrm{X}$ and $\mathrm{Y}$ regions constituting the rich club in the macaque and the human respectively, the overlap was calculated as the ratio of the intersection and the union of regions $\mathrm{X}$ and $\mathrm{Y}$. Significance was assessed with randomization statistics. For each level $\mathrm{k}$ a number of regions equal to the number of $\mathrm{X}$ and $\mathrm{Y}$ was drawn randomly with a uniform probability from the $82 \mathrm{RM}$ regions for the macaque and the human separately. Subsequently, the overlap between this randomly drawn set of regions was calculated. This procedure was repeated 10000 times. Note that the highest overlap is observed for $\mathrm{k}$ values corresponding to the pick of the normalized $\mathrm{RCC}$ for the $\mathrm{MC}$ and $\mathrm{HC}$ ( $\mathrm{k}=56$ for macaque and $\mathrm{k}=55$ for human) (see Fig. 3).

Control analyses The calculation of ED, HCS, HMIS, and RCC from the scrambled MC and $\mathrm{HC}$ revealed that the results are robust. More specifically, the ED remained significant, and the HCS and HMIS remained significant for a large amount of regions (Supp Fig 2). Lastly, the RCC remained significant and closely following the RCC values obtained from the unscrambled networks (Supp Fig 3).

The control analysis of random insertion of connections also led to similar results as the aforementioned control analysis pinpointing the robustness of the initial analysis (data not shown).

The usage of a different parcellation scheme, i.e. BB47, for assembling the MC and $\mathrm{HC}$ led to significant and highly converging results as the ones obtained for the RM since all the analysis results were comparable (data not shown). Conjointly, the above indicate the robustness and independence of the results from particular parcellation schemes.

\section{$\underline{\text { Discussion }}$}

In the current study we adopted a rigid quantitative approach for the comparative examination of the whole brain connectivity of the macaque and the human brain. While similarities between the species were observed, lack thereof might suggest the rewiring of certain regions. Moreover, our analysis revealed topological evolutionary precursors of the human brain. Below we elaborate on key aspects of our results. 


\section{Chapter 4}

Association cortices of the macaque and human brain

Frontal and parietal cortices are commonly placed under the term "association cortices" (Mesulam, 1998, Achard et al., 2007). Our results suggest different evolutionary aspects of their connectivity.

An early view on the frontal cortex (FC) suggests that it has been expanded in the lineage leading to humans (Campbell, 1905; Brodmann, 1912). Expansion of the human FC relative to the macaque FC is supported by contemporary investigations (Van Essen and Dierker, 2007) and is linked to unique human cognitive processes (Barbey et al., 2012). Moreover, FC connectivity changes have also been proposed to underlie unique cognitive processes in humans (Semendeferi et al., 2002). What evidence for the FC connections of the human and macaque exist and do they suggest connectivity reconfigurations between the species? On the one hand, pronounced changes are reported for the arcuate and inferior fronto-occipital fasciculi of humans and macaques (Rilling et al., 2008; Thiebaut de Schotten et al., 2012). On the other hand, a recent review (Petrides et al., 2012) as well as functional (Goulas et al., 2012) and structural (Thiebaut de Schotten et al., 2012) connectivity studies, also suggest comparable connections of the FC of the two species. Previous quantitative analysis has revealed similar connectivity of macaque and human FC regions with a small set of cortical regions (Croxson et al., 2005). Our study extends such finings by taking into account the whole cortex in both species and hence revealing the similarity of connections of FC by examining the totality of the $\mathrm{MC}$ and $\mathrm{HC}$. Our results complement the aforementioned evidence and through a direct whole brain quantitative analysis suggest a statistically significant preservation of the connectivity of, especially lateral, FC regions across the species (Fig. 2). Hence, the relation between the human FC and unique human cognitive processes/intelligence, if any, does not seem to rely on extensive reconfigurations of its connectivity in humans when compared to macaques.

The parietal cortex in macaques and humans consists of distinct subregions that are discernible on functional, connectional and microstructural criteria (von Bonin and Bailey, 1947; Mars et al., 2011; Orban et al., 2006). Qualitative data from fMRI and connectivity studies reveal similarities but also some differences of the functional and connectional architecture of the parietal cortex of the two species (Orban et al., 2006; Mars et al., 2011; Caspers et al., 2011). More specifically, functional differences of intraparietal subregions are discernible and such differences are associated with threedimensional structure from motion (Orban et al., 2006). Additionally, the functional connectivity of the superior parietal and intraparietal regions in the two species with a subset of target regions appears comparable in both humans and macaques, while discrepancies are observed for the inferior lobule (Mars et al., 2011). Additionally, based on a review of lesion, single cell recordings and connectivity data, it has been proposed that while the superior parietal lobule seems to be homologous between the two species, the inferior lobule exhibits several discrepancies (Caminiti et al., 2010). Our whole brain quantitative analysis offer complementary evidence by revealing that the parietal regions fail to reach consistently statistically significant connectivity pattern similarity (Table 1, Fig. 2). More specifically, only the HCS appears significant for the inferior parietal and intraparietal cortex, while the rest of the parietal cortex fails to reach significance for both HCS and HMIS, suggesting a possible rewiring during primate evolution. This lack of similarity can underlie the fact that parietal lesions in the macaque often deviate from effects in humans (Caminiti et al., 2010). Hence, caution is needed when findings from the macaque parietal cortex are translated to humans. 
Centrality and clustering of the macaque and human brain

Our study reveals that the regions in both species do not seem to exhibit a significant level of similarity of their centrality, functioning as a measure of integration, and their clustering, functioning as a measure of segregation (Supp Table 2, Supp Fig. 1). More specifically, the fact that both the $\mathrm{BC}, \mathrm{EC}, \mathrm{CC}$ of the regions in both species do not significantly correlate, indicate that the topological embedding of the regions is not preserved across the species. This is also evident when taking into account the "hubs", the most central regions, where a large mismatch is observed between the MC and $\mathrm{HC}$ (Supp Table 2). However, such discrepancy might arise due to methodological limitations (see Limitations and future directions).

Interestingly, the bilateral CCp, along with the left PCip, was consistently ranked as a hub in both species (Supp Table 2). The CCp has been identified as a major node of the default mode network in both species (Andrews-Hanna, 2010; Mantini et al., 2011). Recent evidence from a functional study in humans suggests that this region exhibits dynamic properties subserving the integration of information from regions of distinct large scale networks (de Pascuale et al., 2012). The centrality of CCp, as well as the fact that it is part of the rich club in both species (Fig. 2, Supp Table 3), might be the structural ground for such a functional property. Consequently, it could be hypothesized that such functional property will also hold for the macaque. However, the CCp, despite being central in both the $\mathrm{MC}$ and $\mathrm{HC}$, also appears to have not retained its connectivity with the rest of the brain across the species (Fig. 2, Table 1). Multimodal imaging of the macaque and human brain might be used to directly address if the aforementioned functional properties of such "topological homologues" are common in the two species or a unique property of the human brain.

Rich clubs: A common structural backbone in the macaque and human brain

Our analysis demonstrates the presence of a rich club organization in both the MC and HC (Fig. 3) in line with previous findings (van den Heuvel and Sporns, 2011; Harriger et al., 2012). Importantly, the results demonstrate that the regions forming a rich club are highly converging across the two species with a significant overlap within the rich club regime (Fig. 3 B, 4). Thus, our comparative approach suggests that this structural backbone is not only present in both macaques and humans, but it also involves highly overlapping regions in the two species. This indicates that it is a structure preserved across primate evolution.

Previous network analysis in the macaque (Harriger et al., 2012) and the human brain (van den Heuvel et al., 2012) revealed that the rich club connections are the most "costly", i.e. span long distances, and mediate traffic between distant regions, through a sequence of short-long-short range structural pathways. Interestingly, studies in the human brain indicate that inter-regional functional interactions depend on physical distance and take place within specific frequency bandwidths (Von Stein and Sarnthein, 2001; Salvador et al., 2005). Additionally, macaque studies have offered preliminary evidence that the dialogue between two cortical regions takes place within specific frequency bandwidths that depend on the laminar structure of the involved regions (Bosman et al., 2012). Our comparative rich club analysis can guide invasive techniques for the functional examination of the regions that appeared to be members of the rich club in the macaque. Such investigation is crucial for assessing if and how the aforementioned factors co-shape the functional dialogue within rich-club and between 


\section{Chapter 4}

rich club and non-rich club regions and the principles that shape the flow of information through this structural backbone. Additionally, such functional investigation might unlock the exact mechanisms underlying the proposed role of rich-club regions in multisensory integration (Zamora- López et al., 2010). Our results, by demonstrating that common regions constitute the rich club in the two species, can be used for translating such functional findings to the human brain and develop hypothesis that ideally would be tested with the use of e.g. electrocorticography. In that way, it could be assessed if "homologous rich club" regions also exhibit comparable functional properties in the two species.

Lesions of rich-club regions have a profound negative impact on the efficiency of the whole brain network and consequently can affect multiple cognitive domains as well as functional aspects like synchronization of large scale functional networks (van den Heuvel and Sporns, 2011). The presence of a rich club structure involving highly overlapping regions in both $\mathrm{MC}$ and $\mathrm{HC}$ suggest that the macaque brain might be used as a model for developing heuristics and predictions, e.g. studying the effects of lesions of "homologous rich club" regions. However, certain common rich club regions lack significant interspecies connectivity similarity (Fig. 2, Fig. 3 B). Computational modelling shows that lesioning a brain region, apart from leading to the expected effects in regions directly connected to it, also leads to global effects (Young et al., 2000). Thus, the same lesion can lead to different global effects, and consequently possibly different behavioural effects, if the wiring of the involved regions differs. By taking into account the above conjointly, we hypothesize that while lesioning rich club regions will have detrimental global effects in both species, the nature and severity of such effects might depend on the degree of preservation of the connectivity of the involved regions.

Factors responsible for connectivity discrepancies between the species

Both genetic and environmental factors underlie the system-level changes, including connectivity, of the cortex of mammals (Krubitzer and Seelke, 2012). For instance, functional connectivity differences observed between the inferior parietal lobule and anterior prefrontal cortex of macaques and humans can be the result of different foraging styles of the two species, dictated by different ecological factors which entail different challenges in decision making (Rushworth et al., 2012; Passingham and Wise, 2012). Our results revealed statistically significant connectivity similarities between humans and macaques while absence thereof might suggest a rewiring caused by the aforementioned factors.

Limitations and future directions

Several limitations should be taken into account when interpreting the findings of our study. First, an a priori parcellation, i.e. the RM, and the expansion model (Van Essen and Dierker, 2007) were used for constructing the MC and HC. Consequently, all the results and interpretations are bound by such a priori assumptions. Our control analysis performed with an alternative parcellation scheme (BB47) revealed highly converging and significant results with the ones obtained from the RM-based analysis. Hence, the choice of the parcellation does not seem to affect the results and conclusions of our study. While the expansion model is used extensively for interspecies comparisons, recent evidence suggests the presence of interspecies functional correspondences not predicted by it (Mantini et al., 2012). In order to perform interspecies comparisons 
without using the expansion model, dwMRI and/or resting-state fMRI data collected in both species in conjunction with sophisticated techniques like network alignment (Berg and Lässig, 2006) can be used for an interspecies connectivity-based region-to-region match. The aforementioned approaches can work in a complementary way since they start from premises that are complementary to the ones constituting the starting point of the current study.

Second, the MC was assembled through a meta-analysis of tracing studies, while the $\mathrm{HC}$ with the aid of dwMRI. The MC was assembled in such a way since tracing studies constitute the gold-standard of structural connectivity and we wanted to exploit the wealth of data available in CoCoMac. Very good correspondence exists between the structural connections as revealed by tracers and diffusion imaging (Hagmann et al., 2008; Schmahmann et al., 2007), but some inconsistencies are also discernible ( $\mathrm{Li}$ et al., 2012), and thus we predict that the usage of a dwMRI for assembling the MC will lead to comparable results.

Third, tractography methods have several limitations. For instance, it has been demonstrated that there is a relation of false-positives and false negatives and connection distance, with longer connection distances appearing more prone to false negatives ( $\mathrm{Li}$ et al., 2012). Hence, connections between distant regions might be underrepresented and might lead to lack of interspecies connectivity similarity. Future studies employing a different modality for the estimation of connectivity in the two species, e.g. resting-state fMRI, will complement the current results.

Fourth, in the current study we compared the large scale inter-regional connectivity of the two species and thus our approach concerns a macroscale level of connectivity (Sporns et al., 2005). Apart from similarities and changes occurring at this level, studies demonstrate that connectivity changes between the species occur at a mesoscale, i.e. connectivity at the laminar level (Preuss, 2000). For instance, while our result indicate preservation of the large scale connectivity of the lateral parts of the FC, reconfigurations at the laminar level seem to have occurred during primate evolution since increased dopaminergic afferents in layers III and V/VI of prefrontal regions were observed for humans and apes relative to macaques (Raghanti et al. 2008). Hence, quantitative comparative studies at multiple levels must take place.

\section{Conclusions}

In summary, we examined in a rigorous way and at the global whole brain level the inter-regional structural connections of the macaque and human brain. While arguing for the suitability of the macaque brain as a model for the human brain, our study also pinpoints discrepancies that should be taken into account. This approach, which can be termed "comparative connectomics", offers closer interspecies comparisons, bringing forth insights concerning evolution of the primate brain at the systems level, and functions as a translational bridge between macaque and human research. Such translational bridge is valuable for clinical, cognitive and systems neuroscience. 


\section{Chapter 4}

\section{$\underline{\text { References }}$}

Achard S, Salvador R, Whitcher B, Suckling J, Bullmore E (2006) A resilient, low-frequency, small-world human brain functional network with highly connected association cortical hubs. J Neurosci 26:63-72.

Alstott J, Breakspear M, Hagmann P, Cammoun L, Sporns O (2009) Modeling the impact of lesions in the human brain. PloS Comput Biol 5:e1000408.

Andrews-Hanna JR, Reidler JS, Sepulcre J, Poulin R, Buckner RL (2010) Functional-anatomic fractionation of the brain's default network. Neuron 65:550-62.

Barbey AK, Colom R, Solomon J, Krueger F, Forbes C (2012) An integrative architecture for general intelligence and executive function revealed by lesion mapping. Brain 135:1154-1164.

Bastiani M, Jon Shah N, Goebel R, Roebroeck A (2012) Human cortical connectome reconstruction from diffusion weighted MRI: The effect of tractography algorithm. NeuroImage 62:1732-1749.

Berg J, Lässig M (2006) Cross-species analysis of biological networks. Proc Nat Acad Sci U S A 103:1096972.

Bezgin G, Vakorin VA, Opstal AJV, Mcintosh AR, Bakker R (2012) Hundreds of brain maps in one atlas: registering coordinate-independent primate neuro-anatomical data to a standard brain. NeuroImage 62:67-76.

Bezgin G, Wanke E, Krumnack A, Kötter R (2008) Deducing logical relationships between spatially registered cortical parcellations under conditions of uncertainty. Neural Networks 21:1132-1145.

Bonacich P (1972) Factoring and weighting approaches to clique identification. J Math Sociol. 2:113-120.

Bosman CA, Schoffelen JM, Brunet N, Oostenveld R, Bastos AM, Womelsdorf T, Rubehn B, Stieglitz T, Weerd PD, Fries P (2012) Attentional stimulus selection through selective synchronization between monkey visual areas. Neuron 75:875-888.

Bressler SL, Menon V (2010) Large-scale brain networks in cognition: emerging methods and principles. Trends Cog Sci 14:277-290.

Brodmann, K (1909) Vergleichende Localisationslehre der Grosshirnrinde in ihren Prinzipien dargestellt auf Grund des Zellenbaues. Leipzig: Barth.

Brodmann K (1912) Neue Ergebnisse über die vergleichende histologische Lokalisation der Grosshirnrinde mit besonderer Berücksichtigung des Stirnhirns. Anat. Anzeiger 41:157-216.

Buckner RL, Sepulcre J, Talukdar T, Krienen FM, Liu H, Hedden T, Andrews-Hanna JR, Sperling R, Johnson K (2009) Cortical hubs revealed by intrinsic functional connectivity: mapping, assessment of stability, and relation to Alzheimer's disease. J Neurosci 29:1860-73.

Burns G, Young MP (2000) Analysis of the connectional organization of neural systems associated with the hippocampus in rats. Philos Trans R Soc Lond B Biol Sci 355:55-70.

Caminiti R, Chafee MV, Battaglia-Mayer A, Averbeck BB, Crowe DA, Georgopoulos AP (2010) Understanding the parietal lobe syndrome from a neurophysiological and evolutionary perspective. Eur $\mathrm{J}$ Neurosci 31:2320-2340.

Campbell, AW (1905) Histological studies on the localisation of cerebral function. Cambridge University Press.

Campbell CBG, Hodos W (1970) The concept of homology and the evolution of the nervous system. Brain Behav. Evol. 3:353-67.

Croxson PL, Johansen-Berg H, Behrens TEJ, Robson MD, Pinsk M, Gross CG, Richter W, Richter MC, Kastner S, Rushworth MFS (2005) Quantitative investigation of connections of the prefrontal cortex in the human and macaque using probabilistic diffusion tractography. J Neurosci 25:8854-66. 
Colizza V, Flammini A, Serrano MA, Vespignani A (2006) Detecting rich-club ordering in complex networks. doi:10.1038/nphys209.

de Pasquale F, Penna SD, Snyder AZ, Marzetti L, Pizzella V, Romani GL, Corbetta M (2012) A cortical core for dynamic integration of functional networks in the resting human brain. Neuron 74:753-764.

Ffytche DH, Catani M (2005) Beyond localization: from hodology to function. Philos Trans R Soc Lond B Biol Sci 360:767-779.

Fillard P, Descoteaux M, Goh A, Gouttard S, Jeurissen B, Malcolm J, Ramirez- Manzanares A, Reisert M, Sakaie K, Tensaouti F, Yo T, Mangin JF, Poupon C (2011) Quantitative evaluation of 10 tractography algorithms on a realistic diffusion MR phantom. NeuroImage 56:220-234.

Freeman LC (1978) Centrality in social networks: conceptual clarification. Soc. Netw. 1:215-239.

Frey S, Campbell JSW, Pike GB, Petrides M (2008) Dissociating the human language pathways with high angular resolution diffusion fiber tractography. J Neurosci 28:11435-44.

Goulas A, Uylings HBM, Stiers P (2012) Unravelling the intrinsic functional organization of the human lateral frontal cortex: a parcellation scheme based on resting state fMRI. J Neurosci 32:10238 -10252.

Hagmann P, Cammoun L, Gigandet X, Meuli R, Honey CJ, Wedeen VJ, Sporns O (2008) Mapping the structural core of human cerebral cortex. PloS Biol 6:e159.

Harriger L, van den Heuvel MP, Sporns O (2012) Rich club organization of macaque cerebral cortex and its role in network communication. PloS One 7:e46497.

Hilgetag CC, Kötter R, Stephan KE, Sporns O (2002) Computational methods for the analysis of brain connectivity, In: Computational Neuroanatomy - Principles and methods (Ascoli GA Ed.) Human Press, pp. 295-335.

Kötter R, Sommer FT (2000) Global relationship between anatomical connectivity and activity propagation in the cerebral cortex. Philos Trans R Soc Lond B Biol Sci 355:127-34.

Kötter R, Wanke E (2005) Mapping brains without coordinates. Philos Trans R Soc Lond B Biol Sci 360:75166.

Krubitzer L, Kaas J (2005) The evolution of the neocortex in mammals: how is phenotypic diversity generated? Curr Opin Neurobiol 15:444-453.

Krubitzer LA, Seelke AMH (2012) Cortical evolution in mammals: The bane and beauty of phenotypic variability. Proc Nat Acad Sci U S A 109:10647-10654.

Li L, Rilling JK, Preuss TM, Glasser MF, Damen FW, Hu X (2012) Quantitative assessment of a framework for creating anatomical brain networks via global tractography. NeuroImage 61:1017-1030.

Mantini D, Gerits A, Nelissen K, Durand J-baptiste, Joly O, Simone L, Sawamura H, Wardak C, Orban GA, Buckner RL, Vanduffel W (2011) Default mode of brain function in monkeys. J Neurosci 31:12954 -12962.

Mantini D, Hasson U, Betti V, Perrucci MG, Romani GL, Corbetta M, Orban GA, Vanduffel W (2012) Interspecies activity correlations reveal functional correspondence between monkey and human brain areas. Nat Methods 9:277-82.

Markov NT, Misery P, Falchier A, Lamy C, Vezoli J, Quilodran R, Gariel MA, Giroud P, Ercsey-Ravasz M, Pilaz LJ, Huissoud C, Barone P, Dehay C, Toroczkai Z, Van Essen DC, Kennedy H, Knoblauch K (2011) Weight consistency specifies regularities of macaque cortical networks. Cereb Cortex 21:1254-72.

Mars RB, Jbabdi S, Sallet J, O’Reilly JX, Croxson PL, Olivier E, Noonan MP, Bergmann C, Mitchell AS, Baxter MG, Behrens TEJ, Johansen-Berg H, Tomassini V, Miller KL, Rushworth MFS (2011) Diffusionweighted imaging tractography-based parcellation of the human parietal cortex and comparison with human and macaque resting-state functional connectivity. J Neurosci 31:4087-4100. 


\section{Chapter 4}

Maslov S, Sneppen K (2002) Specificity and stability in topology of protein networks. Science 296:910-913.

Mesulam MM (1998) From sensation to cognition. Brain 121:1013-52.

Modha DS, Singh R (2010) Network architecture of the long-distance pathways in the macaque brain. Proc Nat Acad Sci U S A 107:13485-90.

Orban G, Van Essen DC, Vanduffel W (2004). Comparative mapping of higher visual areas in monkeys and humans. Trends Cog Sci 8:315-24.

Orban G, Claeys K, Nelissen K, Smans R, Sunaert S, Todd J, Wardak C, Durand J, Vanduffel W (2006). Mapping the parietal cortex of human and non-human primates. Neuropsychologia 44:2647-2667.

Passingham RE, Stephan KE, Kötter R (2002) The anatomical basis of functional localization in the cortex. Nat Rev Neurosci 3:606-16.

Passingham RE (2009) How good is the macaque monkey model of the human brain? Curr Opin Neurobiol 19:6-11.

Passingham RE, Wise SP (2012) The neurobiology of the prefrontal cortex: anatomy, evolution and the origin of insight. Oxford Press.

Petrides M, Tomaiuolo F, Yeterian EH, Pandya DN (2012) The prefrontal cortex: Comparative architectonic organization in the human and the macaque monkey brains. Cortex 48:46-57.

Preuss TM (2000) Taking the measure of diversity: comparative alternatives to the model-animal paradigm in cortical neuroscience. Brain Behav Evol 55:287-299.

Rubinov M, Sporns O (2010) Complex network measures of brain connectivity: uses and interpretations. NeuroImage 52:1059-69.

Raghanti MA, Stimpson CD, Marcinkiewicz JL, Erwin JM, Hof PR, Sherwood CC (2008) Cortical dopaminergic innervation among humans, chimpanzees and macaque monkeys: a comparative study. Neuroscience 155:203-220.

Rilling JK, Glasser MF, Preuss TM, Ma X, Zhao T, Hu X (2008) The evolution of the arcuate fasciculus revealed with comparative DTI. Nat Neurosci 11:2007-2009.

Rushworth MFS, Kolling N, Mars RB (2012) Valuation and decision-making in frontal cortex: one or many serial or parallel systems? Curr Opin Neurobiol 22:1-10.

Salvador R, Suckling J, Schwarzbauer C, Bullmore E (2005) Undirected graphs of frequency-dependent functional connectivity in whole brain networks. Philos Trans R Soc Lond B Biol Sci 360:937-46.

Schmahmann JD, Pandya DN, Wang R, Dai G, D'Arceuil HE, Crespigny AJ de, Wedeen VJ (2007) Association fibre pathways of the brain: parallel observations from diffusion spectrum imaging and autoradiography. Brain 130:630-53.

Semendeferi K, Lu A, Schenker N, Damasio H (2002) Humans and great apes share a large frontal cortex. Nat Neurosci 5:272-6.

Sporns O, Tononi G, Ko R (2005) The Human Connectome: a structural description of the human brain. PloS Comput Biol 1:e42.

Stephan KE, Zilles K, Kötter R (2000) Coordinate-independent mapping of structural and functional data by objective relational transformation (ORT). Philos Trans R Soc Lond B Biol Sci 355:37-54.

Supekar K, Musen M, Menon V (2009) Development of large-scale functional brain networks in children. PloS Biol 7:e1000157.

Sporns O, Zwi J (2004) The small world of the cerebral cortex. Neuroinformatics 2:145-162. 
Sporns O, Honey CJ, Kötter R (2007) Identification and classification of hubs in brain networks. PloS One $10: 31049$.

Thiebaut de Schotten M, Dell'Acqua F, Valabregue R, Catani M (2012) Monkey to human comparative anatomy of the frontal lobe association tracts. Cortex 48:82-96.

Tournier JD, Calamante F, Connelly A (2007) Robust determination of the fibre orientation distribution in diffusion MRI: non-negativity constrained super-resolved spherical deconvolution. NeuroImage 35:14591472 .

Trusina A, Sneppen K, Dodd IB, Shearwin KE, Egan JB (2005) Functional alignment of regulatory networks: a study of temperate phages. PloS Comput Biol 7:e74.

Tzourio-Mazoyer N, Landeau B, Papathanassiou D, Crivello F, Etard O, Delcroix N, Mazoyer B, Joliot M (2002) Automated anatomical labelling of activations in SPM using a macroscopic anatomical parcellation of the MNI MRI single-subject brain. NeuroImage 15:273-89.

Uylings HBM, Van Eden CG (1990) Qualitative and quantitative comparison of the prefrontal cortices in rat and in primates, including humans. Prog Brain Res 85:31-62.

van den Heuvel MP, Mandl RCW, Stam CJ, Kahn RS, Hulshoff Pol HE (2010) Aberrant frontal and temporal complex network structure in schizophrenia: a graph theoretical analysis. J Neurosci 30:15915-26.

van den Heuvel MP, Sporns O (2011) Rich-club organization of the human connectome. J Neurosci 31:1577515786.

van den Heuvel MP, Kahn RS, Goñi J, Sporns O (2012) High-cost, high-capacity backbone for global brain communication. Proc Nat Acad Sci U S A 109:11372-11377.

Van Essen DC (2004) Surface-based approaches to spatial localization and registration in primate cerebral cortex. NeuroImage 23:97-107.

Van Essen DC, Dierker DL (2007) Surface-based and probabilistic atlases of primate cerebral cortex. Neuron 56:209-25.

Von Bonin GV, Bailey P (1947) The neocortex of macaca mulatta. The University of Illinois Press, Urbana, Illinois.

Von Stein A, Sarnthein J (2000) Different frequencies for different scales of cortical integration: from local gamma to long range alpha/theta synchronization. Intern J Psychophysiol 38:301-13.

Walker AE (1940) A cytoarchitectural study of the prefrontal area of the macaque monkey. J Comp Neurol 73: $59-86$.

Watts DJ, Strogatz SH (1998) Collective dynamics of “small-world” networks. Nature 393:440-442.

Young MP, Hilgetag C-c, Scannell JW (2000) On imputing function to structure from the behavioural effects of brain lesions. Philos Trans R Soc Lond B Biol Sci 355:147-161.

Zamora-López G, Zhou C, Kurths J (2010) Cortical hubs form a module for multisensory integration on top of the hierarchy of cortical networks. Front Neuroinf 4:1-12.

Zuo XN, Ehmke R, Mennes M, Imperati D, Castellanos FX, Sporns O, Milham MP (2012) Network centrality in the human functional connectome. Cereb Cortex 22:1862-1875. 
Chapter 4

\section{Supplementary material}

A

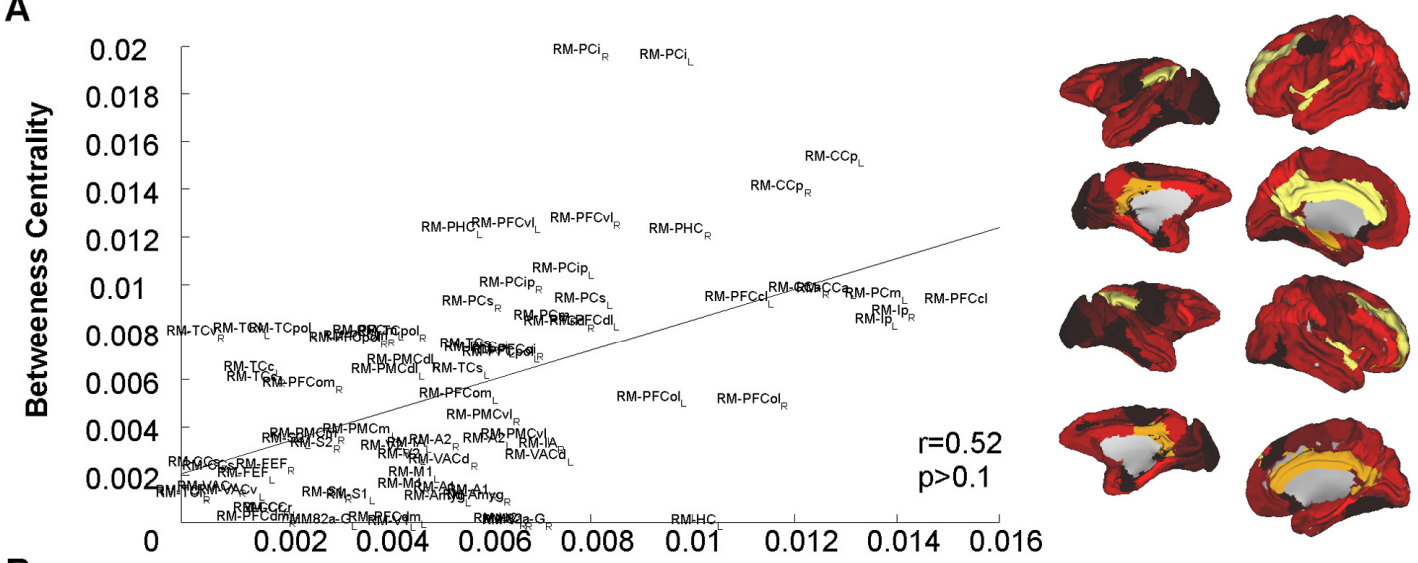

B

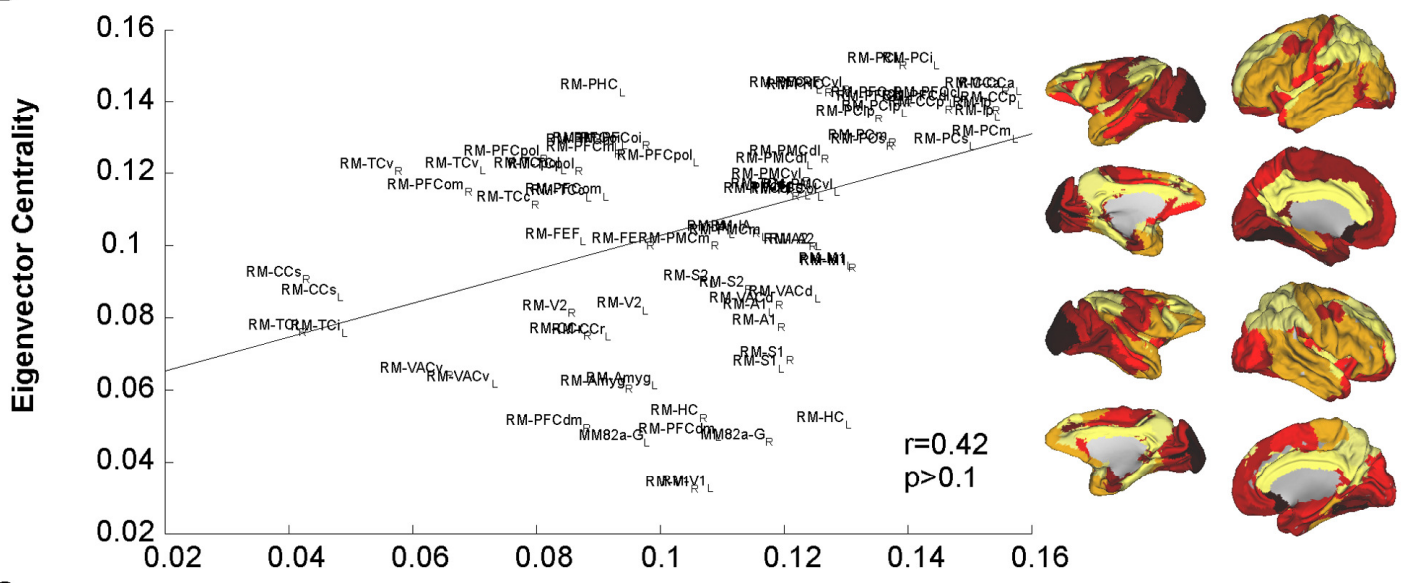

C

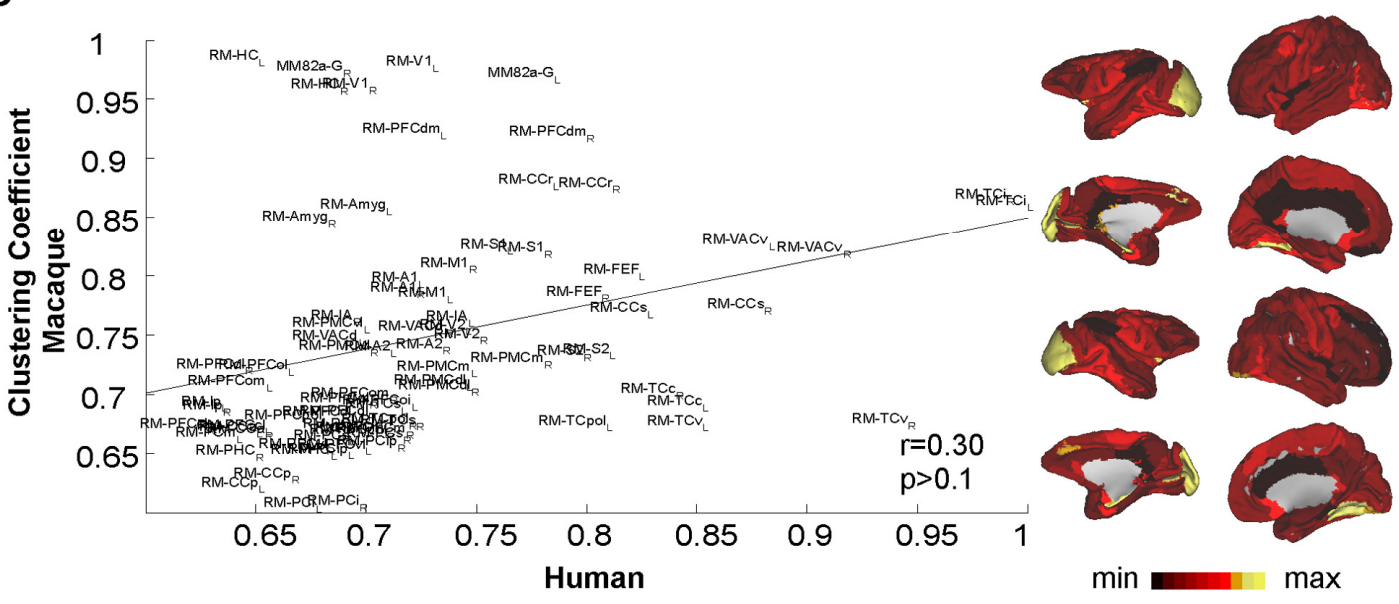

Supp Figure 1. Scatter plots and renderings depicting A-B. The centrality, quantified with the BC and EC, and C. the segregation, quantified with the $\mathrm{CC}$, of the brain regions in the two species. Despite a medium positive correlation, all correlations were not significant when compared to the values obtained from random networks matched for node, edge and degree distribution. 
A

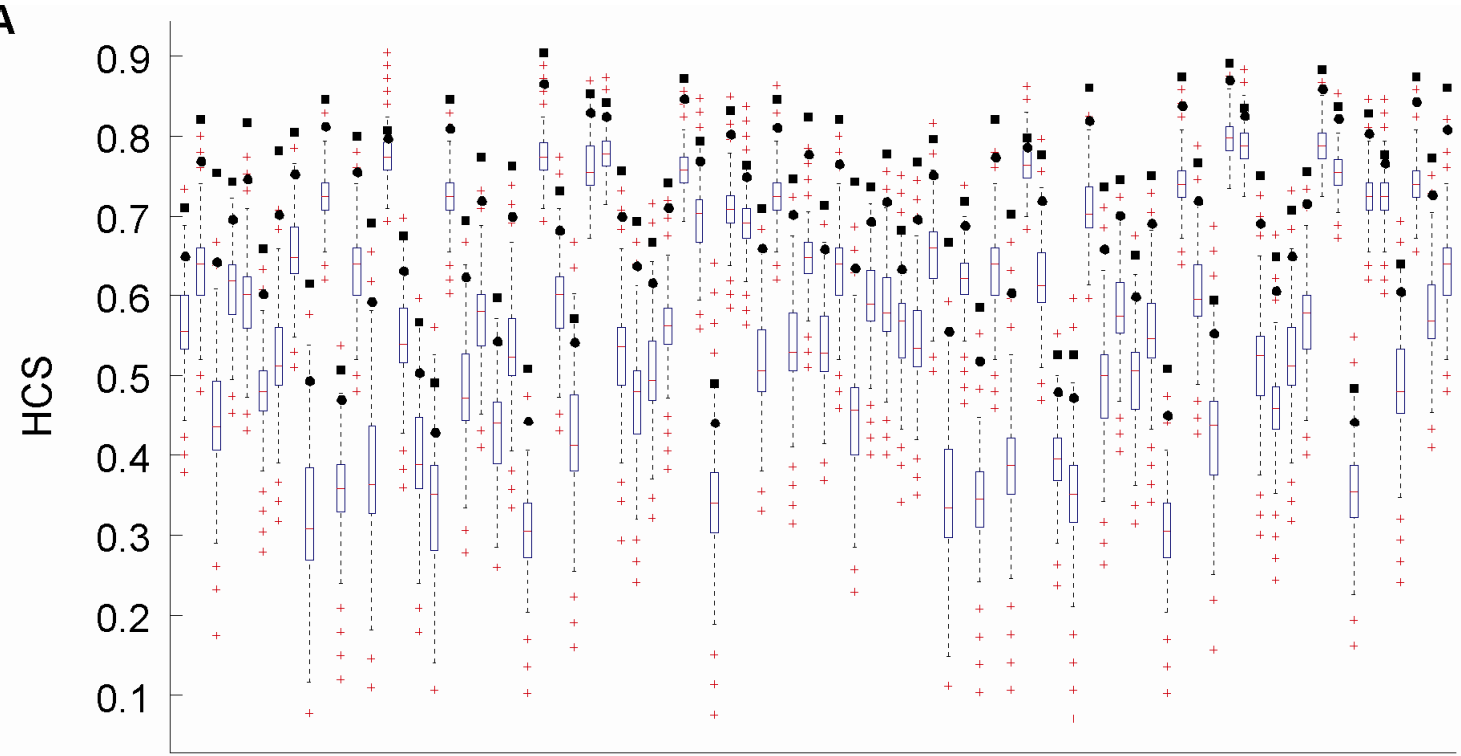

B

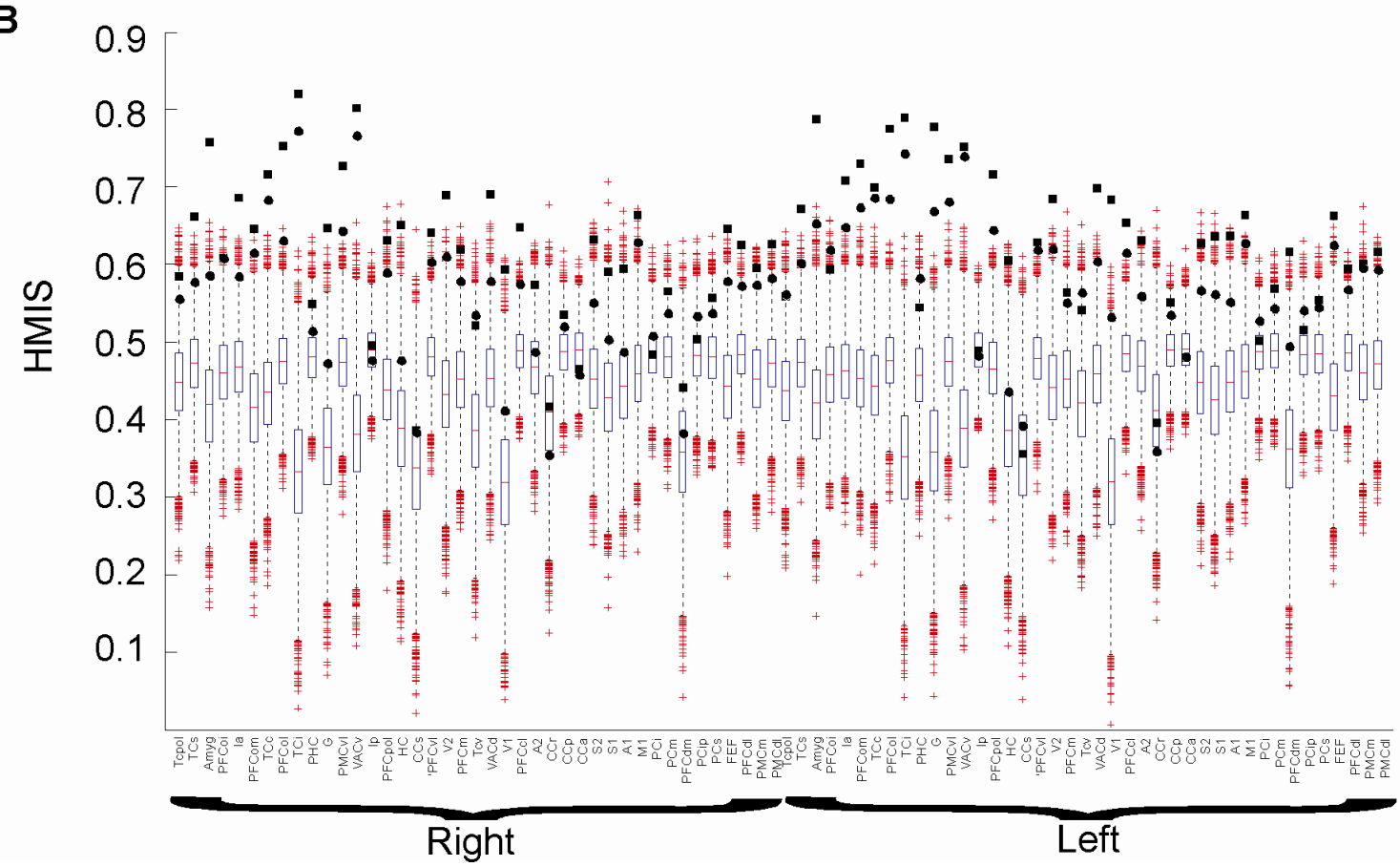

Supp Figure 2. Region-wise values obtained for A. HCS and B. HMIS. Black squares and black circles represent the values obtained from the unscrambled and scrambled MC and HC respectively. Boxplots represent the null values obtained from matched random networks and depict the median, $25 \%$ and $75 \%$ quintiles, and outliers of the null values. 
Chapter 4
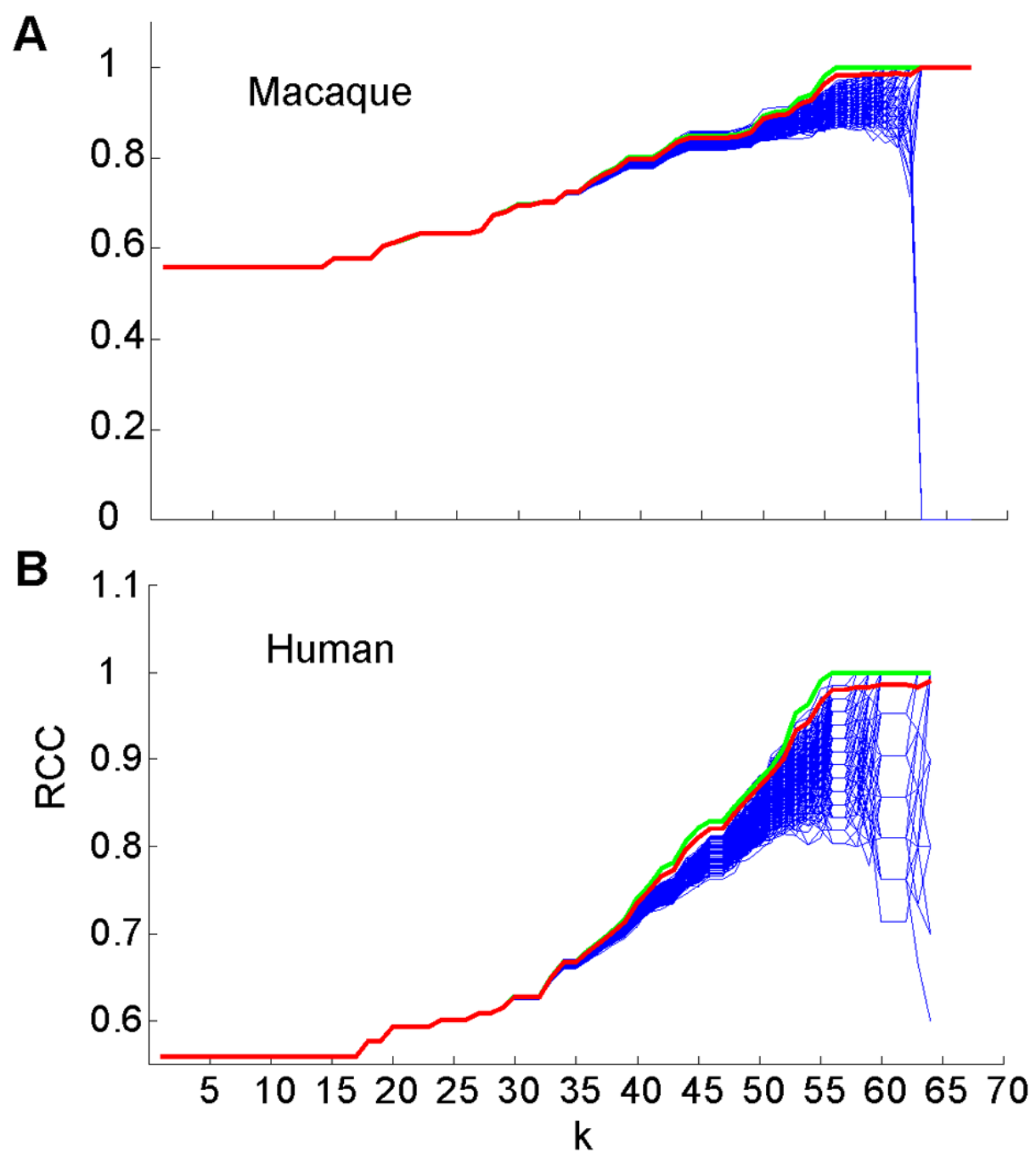

Supp Figure 3. Curves depicting the values for the RCC for a range of k values for both A. macaque and B. human. Green curves and red curves correspond to values obtained from the unscrambled and scrambled networks respectively. Blue curves correspond to values obtained from matched random networks. Note that both the green and red curves lie above the blue ones that represent the null values for the RCC. 
Supp Table 1. RM acronyms and full names of the regions constituting the whole brain parcellation used in the current study.

\begin{tabular}{|c|c|c|c|}
\hline $\begin{array}{l}\text { RM } \\
\text { acronyms }\end{array}$ & Full name & $\begin{array}{c}\text { Cortical } \\
\text { Region }\end{array}$ & Cortical Type \\
\hline Tcpol & temporal polar cortex & $\mathrm{T}$ & Iso \\
\hline $\mathrm{TCs}$ & superior temporal cortex & $\mathrm{T}$ & Iso \\
\hline Amyg & amygdala & $\mathrm{L}$ & Sub \\
\hline PFCoi & orbito intermediate prefrontal cortex & $\mathrm{F}$ & Iso \\
\hline la & anterior insula & $\mathrm{I}$ & Iso \\
\hline PFCom & orbitomedial prefrontal cortex & $\mathrm{F}$ & Iso \\
\hline $\mathrm{TCc}$ & central temporal cortex & $\mathrm{T}$ & Iso \\
\hline PFCol & orbitolateral prefrontal cortex & $\mathrm{F}$ & Iso \\
\hline $\mathrm{TCi}$ & inferior temporal cortex & $\mathrm{T}$ & Iso \\
\hline $\mathrm{PHC}$ & parahippocampal cortex & $\mathrm{L}$ & Allo \\
\hline G & gustatory cortex & $\mathrm{F}$ & Iso \\
\hline PMCvl & ventrolateral premotor cortex & $\mathrm{F}$ & Iso \\
\hline VACV & anterior visual area, ventral part & 0 & Iso \\
\hline Ip & posterior insula & I & Iso \\
\hline PFCpol & prefrontal polar cortex & $\mathrm{F}$ & Iso \\
\hline $\mathrm{HC}$ & hippocampus & $\mathrm{L}$ & Allo \\
\hline $\mathrm{CCs}$ & subgenual cingulate cortex & $\mathrm{L}$ & Allo \\
\hline PFCvl & ventrolateral prefrontal cortex & $\mathrm{F}$ & Iso \\
\hline V2 & visual area 2 (secondary visual cortex) & 0 & Iso \\
\hline $\mathrm{PFCm}$ & medial prefrontal cortex & $\mathrm{F}$ & Iso \\
\hline TCV & ventral temporal cortex & $\mathrm{T}$ & Iso \\
\hline VACd & anterior visual area, dorsal part & $\mathrm{O}$ & Iso \\
\hline V1 & visual area 1 (primary visual cortex) & 0 & Iso \\
\hline $\mathrm{PFCcl}$ & centrolateral prefrontal cortex & $\mathrm{F}$ & Iso \\
\hline A2 & secondary auditory cortex & $\mathrm{T}$ & Iso \\
\hline $\mathrm{CCr}$ & retrosplenial cingulate cortex & $\mathrm{L}$ & Allo \\
\hline $\mathrm{CCp}$ & posterior cingulate cortex & $\mathrm{L}$ & Allo \\
\hline $\mathrm{CCa}$ & anterior cingulate cortex & $\mathrm{L}$ & Allo \\
\hline S2 & secondary somatosensory cortex & $\mathrm{P}$ & Iso \\
\hline S1 & primary somatosensory cortex & $\mathrm{P}$ & Iso \\
\hline A1 & primary auditory cortex & $\mathrm{T}$ & Iso \\
\hline M1 & primary motor cortex & $\mathrm{F}$ & Iso \\
\hline $\mathrm{PCi}$ & inferior parietal cortex & $\mathrm{P}$ & Iso \\
\hline $\mathrm{PCm}$ & medial parietal cortex & $\mathrm{P}$ & Iso \\
\hline PFCdm & dorsomedial prefrontal cortex & $\mathrm{F}$ & Iso \\
\hline PCip & intraparietal cortex & $\mathrm{P}$ & Iso \\
\hline $\mathrm{PCs}$ & superior parietal cortex & $\mathrm{P}$ & Iso \\
\hline FEF & frontal eye field & $\mathrm{F}$ & Iso \\
\hline PFCdl & dorsolateral prefrontal cortex & $\mathrm{F}$ & Iso \\
\hline $\mathrm{PMCm}$ & medial premotor cortex & $\mathrm{F}$ & Iso \\
\hline PMCdl & dorsolateral premotor cortex & $\mathbf{F}$ & Iso \\
\hline
\end{tabular}

Each region is classified as allocortical (allo), isocortical (iso) or subcortical (sub). An assignment is also provided based on a 5 way division $(\mathrm{F}=$ frontal, $\mathrm{T}=$ temporal, $\mathrm{P}=$ parietal, $\mathrm{O}=$ occipital, $\mathrm{L}=$ limbic, $\mathrm{I}=$ insular). 


\section{Chapter 4}

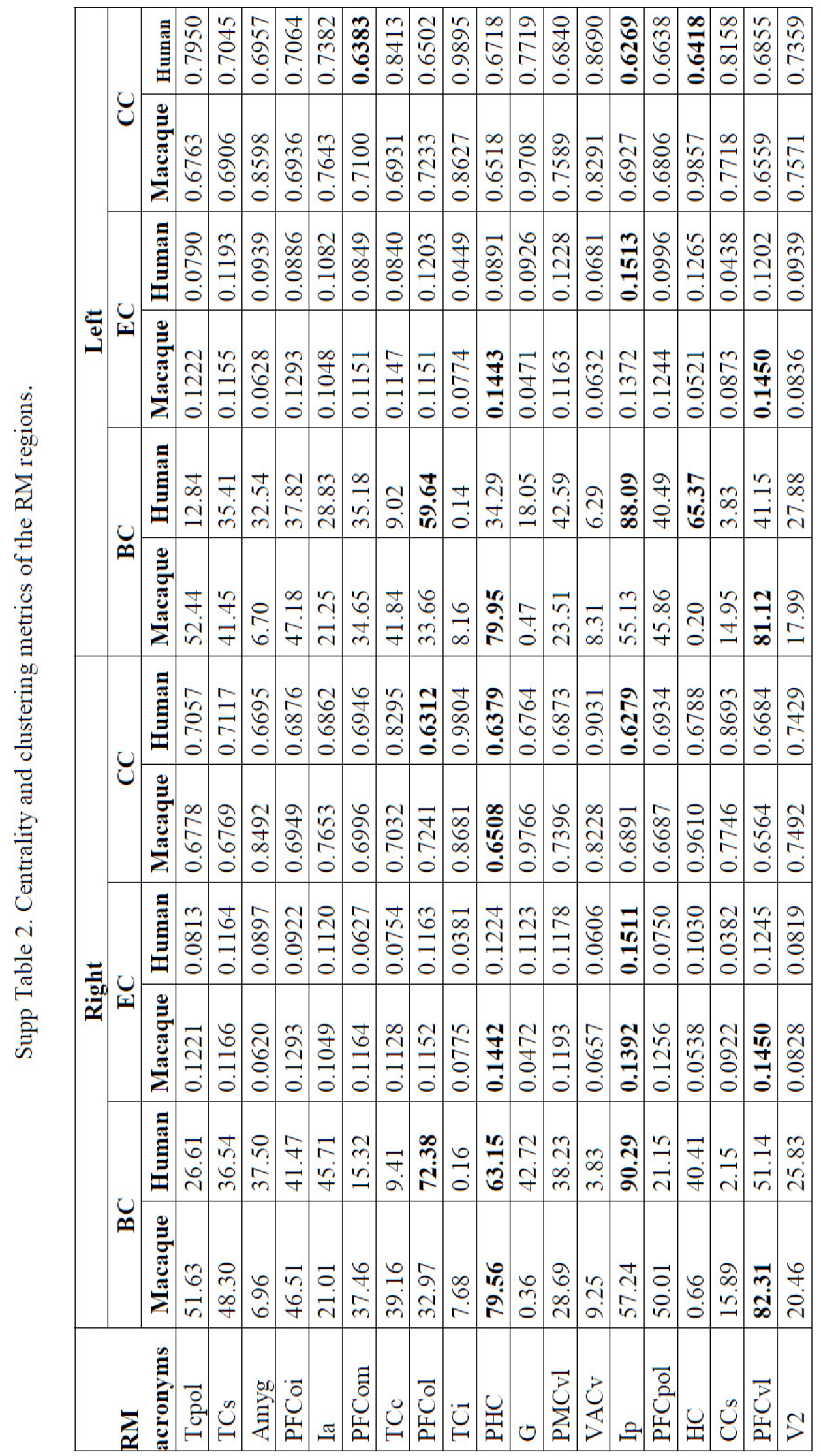




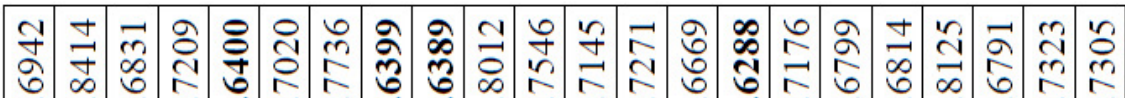

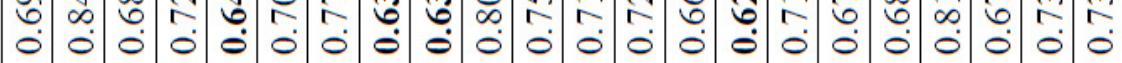

-

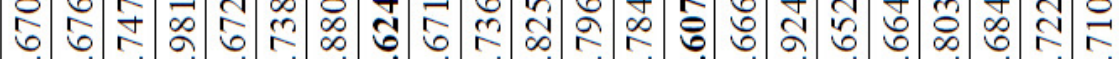
$\begin{array}{llllllllllllllllllllll}0 & 0 & 0 & 0 & 0 & 0 & 0 & 0 & 0 & 0 & 0 & 0 & 0 & 0 & 0 & 0 & 0 & 0 & 0 & 0 & 0 & 0\end{array}$

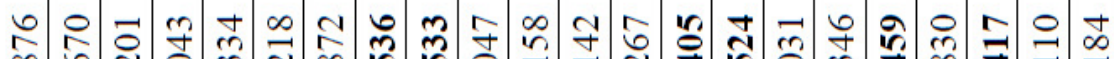

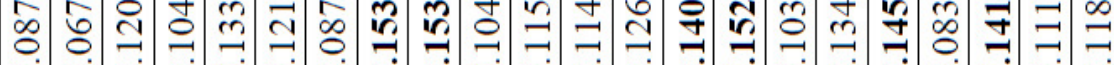

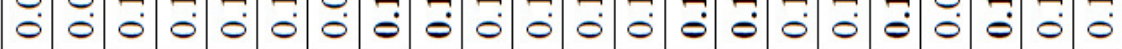

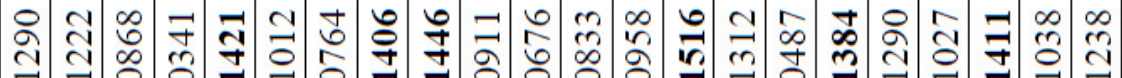

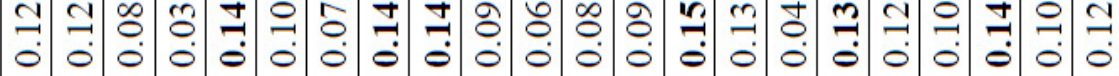

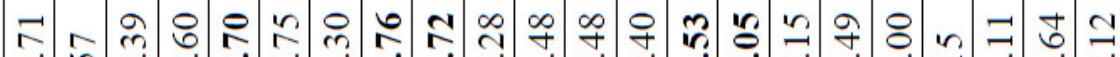
๗઼

实去。 म

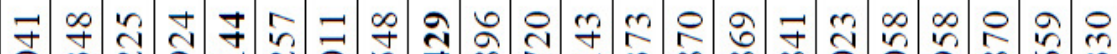
实

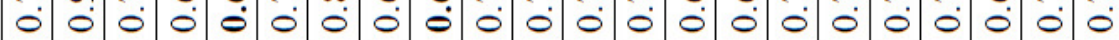

ஓ 6.

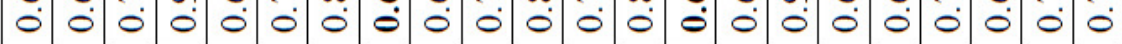
수일 立

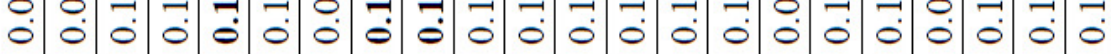

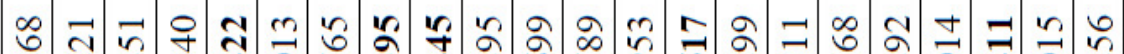

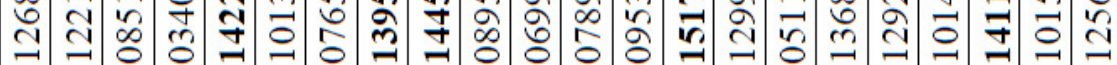

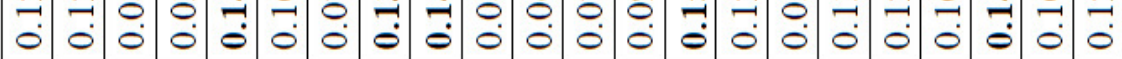

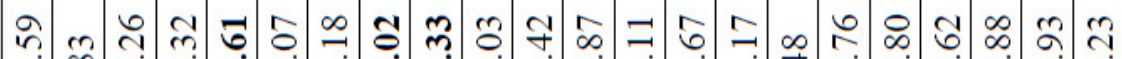

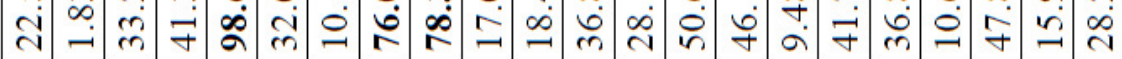

ஸิ

nं

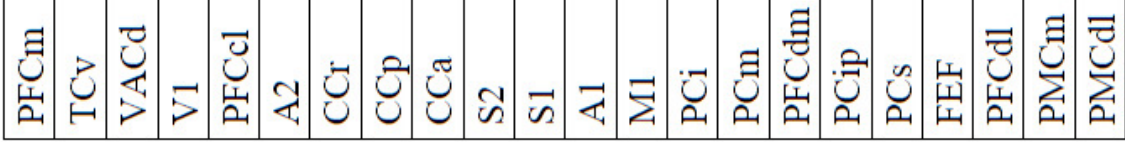

$\neg \widetilde{Q}$

켤

记

(1)

决

킁키

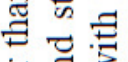

-

햌용

\%

祍寻

릉표

今正

응 긍

붕의

当은

的茫

$\pm-2$

를

紊寻安

그음

잏

要

品㤩总

गु 늬

के च

으눙

름

氠

๑ 0.

可灵

胥讪䒕

킄일

능 讪冚

๑)

쿜.

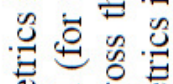

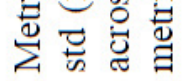




\section{Chapter 4}

Supp Table 3. List of regions constituting the rich club in macaque and human.

\begin{tabular}{|c|c|c|c|c|}
\hline & \multirow[b]{2}{*}{$\begin{array}{l}\text { RM } \\
\text { acronyms }\end{array}$} & \multicolumn{3}{|c|}{ Coordinates of centre of mass } \\
\hline & & $\mathrm{x}$ & $\mathrm{y}$ & $\mathbf{z}$ \\
\hline Macaque & PHC_R & 13.26 & -20.92 & -7.10 \\
\hline & Ip_R & 21.30 & -4.81 & $-1,10$ \\
\hline & PFCVl R & 19.31 & 9.24 & 7.95 \\
\hline & PFCcl_R & 11.41 & 15.55 & 12.90 \\
\hline & CCp_R & 3.52 & -21.30 & 9.10 \\
\hline & CCa_R & 2.46 & 1.66 & 12.80 \\
\hline & PCi_R & 16.73 & -22.96 & 16.80 \\
\hline & PCip_R & 9.15 & -23.75 & 13.90 \\
\hline & PFCdI_R & 6.40 & 13.91 & 18.30 \\
\hline & PHC_L & -13.02 & -20.78 & -7.91 \\
\hline & Ip_L & -21.42 & -2.39 & -1.19 \\
\hline & PFCvI_L & -20.10 & 9.86 & 8.90 \\
\hline & PFCcl_L & -12.10 & 16.47 & 12.10 \\
\hline & CCp_L & -4.23 & -20.98 & 8.44 \\
\hline & CCa_L & -3.54 & 4.11 & 13.00 \\
\hline & PCi_L & -17.30 & -23.33 & 16.10 \\
\hline & PCm_L & -3.40 & -26.52 & 14.90 \\
\hline & PCip_L & -10.65 & -22.37 & 13.80 \\
\hline & PFCdl_L & -7.58 & 14.20 & 17.40 \\
\hline Human & Ip_R & 42.83 & -4.10 & 1.42 \\
\hline & PFCcl_R & 25.43 & 40.83 & 34.79 \\
\hline & CCp_R & 9.92 & -48.92 & 26.33 \\
\hline & CCa_R & 6.00 & 13.65 & 30.61 \\
\hline & PCi_R & 41.62 & -53.39 & 42.95 \\
\hline & PFCdl_R & 24.80 & 12.89 & 53.77 \\
\hline & Ip_L & -42.92 & -4.18 & 1.28 \\
\hline & PFCcl_L & -25.44 & 40.87 & 34.80 \\
\hline & CCp_L & -9.86 & -48.90 & 26.30 \\
\hline & CCa_L & -5.68 & 13.53 & 30.18 \\
\hline & PCi_L & -41.67 & -53.46 & 43.03 \\
\hline & PCm_L & -8.34 & -56.88 & 39.65 \\
\hline & PCip_L & $-22,64$ & -62.00 & 56.03 \\
\hline & PCs_L & -13.93 & -52.85 & 60.79 \\
\hline & PFCdl_L & -24.70 & 13.16 & 53.91 \\
\hline
\end{tabular}

The set of regions corresponds to the level $\mathrm{k}$ for which the maximum normalized $\mathrm{RCC}$ was observed $(\mathrm{k}=56$ for macaque and $\mathrm{k}=55$ for human) (see Fig. 3). Regions in bold denote rich club regions common in both species. Coordinates for the macaque correspond to the 199 space and for the human to MNI space. The suffixes $\mathrm{R}$ and $\mathrm{L}$ denote the right and left hemisphere. 


\section{Chapter 5}

\section{Maturation of task-induced brain activation and long range functional connectivity in adolescence revealed by multivariate pattern classification}

Keulers EHH, Goulas A, Jolles J, Stiers P (2012) Maturation of task-induced brain activation and long range functional connectivity in adolescence revealed by multivariate pattern classification. NeuroImage 60:1250-1265. 


\begin{abstract}
The present study uses multivariate pattern classification analysis to examine maturation in task-induced brain activation and in functional connectivity during adolescence. The multivariate approach allowed accurate discrimination of adolescent boys of respectively 13, 17 and 21 years old based on brain activation during a gonogo task, whereas the univariate statistical analyses showed no or only very few, small agerelated clusters. Developmental differences in task activation were spatially distributed throughout the brain, indicating differences in the responsiveness of a wide range of task-related and default mode regions. Moreover, these distributed age-distinctive patterns generalized from a simple gonogo task to a cognitively and motivationally very different gambling task, and vice versa. This suggests that functional brain maturation in adolescence is driven by common processes across cognitive tasks as opposed to taskspecific processes. Although we confirmed previous reports of age-related differences in functional connectivity, particularly for long range connections $(>60 \mathrm{~mm})$, these differences were not specific to brain regions that showed maturation of task-induced responsiveness. Together with the task-independency of brain activation maturation, this result suggests that brain connectivity changes in the course of adolescence affect brain functionality at a basic level. This basic change is manifest in a range of tasks, from the simplest gonogo task to a complex gambling task.
\end{abstract}




\section{$\underline{\text { Introduction }}$}

Adolescence is a developmental period, between childhood and adulthood, of profound changes in body and behaviour. These changes also include increased risk taking behaviour, leading to rising numbers of accidents and early death, and an increased incidence of behavioral disorders in adolescence (Casey et al., 2008; Dahl, 2004; Steinberg, 2008). Although the neurobiological mechanisms underlying these changes are not yet well understood, it is likely that prolonged myelination and synaptic pruning play a role. Post-mortem studies have shown that these neural maturation processes continue well into young adulthood (Glantz et al., 2007; Huttenlocher and Dabholkar, 1997; Yakovlev and Lecours, 1967). Synaptic pruning is thought to underlie the decrease in gray matter density and cortical thickness observed in MRI studies, particularly in more complex association cortices (Giedd, 2004; Gogtay et al., 2004; Sowell et al., 2004). Myelination, on the other hand, is commonly associated with the linear increase in white matter volume seen in structural MRI studies (Giedd, 2004) and the protracted maturation of white matter bundles measured with diffusion weighted MRI (Lebel et al., 2008).

These anatomical changes are paralleled by wide-spread changes in functional brain organization. Brain-wide patterns of low- frequency temporal correlations in the fMRI signal during rest have shown a shift from childhood to adulthood in the strength of functional connectivity from short range toward long range connections (Fair et al., 2009; Kelly et al., 2009). Graph Theory analyses of whole brain networks have shown that network efficiency does not change with age, but that in childhood brain networks are organized more locally, based on anatomical proximity, whereas adult brain networks comprise regions spread over different brain lobes (Fair et al., 2009; Power et al., 2010; Vogel et al., 2010). Many developmental fMRI studies have shown that taskrelated brain activity also undergoes changes during the transition from childhood to adulthood (for reviews, see Berl et al., 2006; Durston and Casey, 2006; Luna et al., 2010).

The most consistent finding for cognitive tasks in the time window of adolescence is a maturation- related increase of BOLD response strength in the brain structures that are associated with task performance in adults (e.g. Crone et al., 2006; Keulers et al., 2011; Rubia et al., 2006). In other brain regions, hypothesized to be uncorrelated to task performance, the magnitude of brain activation tends to decrease or even disappear with age (e.g. Brown et al., 2005; Durston et al., 2006; Rubia et al., 2006). These age-related differences in response strength of particular brain areas during task execution are thought to be the consequence of brain-wide maturational changes in the way of interactions between neuron populations (e.g. Luna et al., 2010; Vogel et al., 2010).

A question fundamental to understanding functional brain maturation is then how the differences in task-induced activation relate to the changes in functional brain organization. It is well-established that there is a close correspondence between functional connectivity patterns, as evidenced from low-frequency BOLD fluctuations during rest as well as task performance, and the patterns of brain activity that emerge contingent upon task execution (e.g. Calhoun et al., 2008; Fox et al., 2006b; Smith et al., 2009). Specifically, the distributed functional organization of cortical areas that emerges during adolescent development largely overlaps with the clusters of commonly activated and deactivated brain areas that are consistently found during performance of a wide range of cognitive tasks (Cole and Schneider, 2007; Dosenbach et al., 2006, 2007; Sridharan et al., 2008). Moreover, in adults there is a close relationship between 


\section{Chapter 5}

the strength of functional connectivity between two brain sites during rest and their response strength modulation over trials during the execution of cognitive tasks (Mennes et al., 2010). Based on this close correspondence we hypothesized that particularly those regions that change their task-induced response strength with age will also show maturation of functional connectivity in the same age range.

This general relationship between changes in functional activation and in connectivity in the course of adolescent maturation may not hold for all task-induced activity, however. During adolescence, maturation is assumed to be associated with higher, more complex cognitive functions. Particularly well-studied are, for instance, response inhibition (e.g. Rubia et al., 2006, 2007; Velanova et al., 2009), working memory ability (e.g. Crone et al., 2006; Geier et al., 2009) and risk taking behavior (e.g. Keulers et al., 2011; Steinberg et al., 2008; van Leijenhorst et al., 2010), among others. For each of these abilities maturation may be associated with changes in specific brain structures involved in the ability and at a specific time window. In line with this, Cohen et al. (2010) were able to predict age from functional activation maps depicting the activation differences between successful stop trials and go trials, but not from the activation associated with go trials compared to rest. This suggests that maturation related differences in brain activity may be function specific.

In the present study, we investigated the patterns of maturation in both functional connectivity estimated from low-frequency BOLD signal fluctuations and functional activation related to task execution. Brain-wide maturation patterns will be quantified with multivariate pattern classification (MVP) tools (for reviews, see Haynes and Rees, 2006; Pereira et al., 2009). These tools use machine learning principles to find the pattern in a multidimensional space (e.g. the voxels of the images) that distinguishes two classes of examples. MVP analysis was introduced in the neuroimaging literature to "read" the voxel response patterns in visual cortex associated with particular stimulus categories (e.g. Cox and Savoy, 2003; Haxby et al., 2001), but has since then also been used across subjects, for instance, to find the distributed pattern that characterizes neuropathology (e.g. Fan et al., 2007; Sun et al., 2009). Here we will use MVP to classify functional brain maps according to age. Sensitivity of MVP to developmental patterns has recently been demonstrated (Cohen et al., 2010; Dosenbach et al., 2010). MVP analysis is generally more sensitive than the traditional univariate general linear model (GLM) analysis (e.g. De Martino et al., 2008). Moreover, MVP allows to directly test the generalizability of a distinctive pattern learned from a particular set of examples to a new set of example data, to establish whether they carry the same agediscriminative information. This feature allows us to directly test the functional specificity of maturation patterns by training a classifier on functional maps derived from a particular task and testing on data acquired during another task.

First of all, we established the sensitivity of the MVP classification to characterize maturation processes that take place during adolescence. We made use of brain activation data during the performance of a gonogo task, obtained from participants in three age groups, respectively 13, 17 and 21 years of age. The gonogo task focuses on neural processes related to the inhibition of a preponent response and has been frequently used in neurodevelopmental studies (e.g. Durston et al., 2006; Rubia et al., 2006; Tamm et al., 2002). In the task used here, participants had to press a response key whenever a letter was presented, but had to withhold their response when the infrequent nogo stimulus was presented (letter "A"). Next to the MVP classification analysis, we applied the traditional univariate voxel- wise analysis to establish agerelated functional differences between the different age groups. 
Secondly, we studied the functional specificity of the maturation patterns found in the task-related brain activity. The generalization step of MVP classification allowed us to address this question in a direct way. Different cognitive tasks intend to steer specific neurocognitive processes. By using data from one particular task for training and data from a completely different task for testing, it can be directly investigated whether the pattern learned is associated with a specific cognitive function unique to the training task. Therefore, we also used fMRI data obtained from the same sample of participants while they performed a challenging gambling task (Keulers et al., 2011) and investigated whether the classifier trained on the data from the simple gonogo task was successful in classifying participants according to age from their functional activation maps during the complex gambling task, and vice versa.

Thirdly, we studied the relationship of task-related maturation patterns to the differences in short and long range low-frequency functional connectivity that have been described from childhood to early adulthood (Fair et al., 2007a, 2008, 2009; Kelly et al., 2009). We determined the strength of connectedness (Bullmore and Sporns, 2009) of gray matter voxels based on low frequency signal fluctuations in the gonogo data. Although low-frequency data obtained during task performance yield small differences in the distribution of functional connectivity compared to data obtained during rest, the overall lay-out of networks between the data is very similar (Dosenbach et al., 2010; Fair et al., 2007b). We investigated whether voxels that contributed to task-related age classification in the MVP differed from other voxels in their functional connectedness with the rest of the brain. In addition, in a whole brain exploratory analysis we entered the short and long range connectivity strength maps into a MVP classification analysis to allow a visualization of the maturation in functional connections and to compare these with maturational patterns in brain activity during task execution.

\section{Materials and methods}

Participants and procedure A total of 70 participants from the seventh (12/13 year-olds) and eleventh (16/17 year-olds) grades of pre-university education as well as university students (20/21 year-olds) were included. This sample was also described in a previous study, which examined developmental differences in brain activation during a challenging gambling task (Keulers et al., 2011). Participants were recruited through advertisements and information talks. All participants had normal or corrected-tonormal vision, were free from psychiatric or neurological abnormalities, never repeated or skipped a grade, did not use medication that could influence cognitive functioning and were screened for MRI contra-indications. Written informed consent was obtained from all participants and their parents in case participants aged 17 or less. Participants received travel expenses and a reimbursement of 25 Euro. The study was approved by the ethical committee of the Faculty Psychology and Neuroscience of the Maastricht University. Data from 1 participant were excluded due to a problem of motivation, 2 participants were excluded due to technical problems, 3 participants due to poor behavioural performance (error rate $>30 \%$ ) and 7 participants due to excessive head motion (see Preprocessing section for details). This resulted in a final sample of 57 males, right- handed participants divided into three age groups as follows: 13 year-olds $(\mathrm{N}=19$, mean age $=12.9, \mathrm{SD}=0.3$, range $12.3-13.7), 17$ year-olds $(\mathrm{N}=21$, mean age $=17.0$, $\mathrm{SD}=0.4$, range $16.3-17.7)$ and 21 year-olds $(\mathrm{N}=17$, mean age $=21.0, \mathrm{SD}=0.6$, range 20.1-21.9). 


\section{Chapter 5}

Participants attended a training session on average one week before the scan session. The training session served to familiarize participants with the scanning environment and procedure, and to practice the experimental tasks using a mock scanner. In addition, the youngest age group received a training in the mock scanner to reduce motion (Slifer et al., 1993). Participants viewed a cartoon video- tape in the mock scanner for $2 \times 10$ min, which was halted whenever the participants moved their head more than $4.5 \mathrm{~mm}$. Furthermore, two neuropsychological tests were administered to obtain estimates of intelligence. Age groups did neither differ on an estimate of verbal intelligence $(\mathrm{M}=113.89, \mathrm{SD}=8.23, \mathrm{~F}(2,54)=2.38 ; \mathrm{p}=.102)$ as assessed with the Peabody Picture Vocabulary Test-III-NL (Dunn and Dunn, 2005) nor on an estimate of nonverbal intelligence $(\mathrm{M}=121.96, \mathrm{SD}=5.17, \mathrm{~F}(2,54)=1.40 ; \mathrm{p}=.256)$ as assessed with the Raven Standard Progressive Matrices (Raven et al., 1998). The Child Behaviour Checklist and the Youth Self Report were administered in the two youngest age groups (Achenbach and Rescorla, 2001), whereas the Adult Self Report (Achenbach and Rescorla, 2003) was presented in the student group to screen for behavioural problems. All participants had scores on the total problem scale of these questionnaires within 1 SD of the mean of a normative standardized sample. Furthermore, age groups did not differ on the total problem scale of the self report versions $(\mathrm{F}(2,52)=1.46 ; \mathrm{p}=.242)$. In addition, level of parental educa- tion was inquired with a commonly-used Dutch education scale (Directoraat-Generaal voor de Arbeidsvoorziening, 1989), as higher levels of parental education have been associated with higher performance on executive functioning tasks (Ardila et al., 2005; Klenberg et al., 2001). Fifteen parent-pairs (26.3\%) had a moderate level of education (elementary school to general secondary education) and $42(73.7 \%)$ parent-pairs had a high level of education (high vocational education to doctoral degree). Level of parental education did not differ between age groups $\left(\chi^{2}(2)=2.66\right.$; $\mathrm{p}=0.264)$.

Gonogo task The gonogo task consisted of three letter stimuli ("X", "B" and "A") that were randomly presented using a fast event-related design. Participants were instructed to press the response key every time a letter appeared on the screen, but to withhold their response when an "A" appeared. The letter stimuli corresponded to three conditions: Go ("X"), Go oddball ("B") and Nogo ("A"). In $60 \%$ of all trials, the letter "X" was shown, whereas the letters " $A$ " and " $B$ " both appeared in $20 \%$ of all trials. The higher percentage of Go stimuli allowed for the build up of a prepotent tendency to respond. The Go oddball stimuli required a response comparable to the Go trials, but occurred as often as the Nogo trials to control for the effect of low-frequent occurrence (Chikazoe et al., 2009; Hampshire et al., 2010). Letters were presented visually at the centre of the screen, with a duration of $200 \mathrm{~ms}$. During interstimulus intervals a fixation cross was shown and jittering was applied to these intervals. The interstimulus interval varied between 1000 and $2000 \mathrm{~ms}$, with an average duration of $1500 \mathrm{~ms}$. In addition, null-events with the same duration and interstimulus interval as the letter trials were included (33\% of all trials) in which the fixation cross remained visible, to allow for

better separation of the condition specific BOLD responses. The task was presented in two runs, each consisting of 260 trials, of which 175 were letters. Duration of each run was $7.48 \mathrm{~min}$. The mean and variability of reaction times, errors of omission (misses) and errors of commission (false alarms) were recorded. The task was programmed using E-Prime (version 1.1; Psychology Software Tools Inc.).

Gambling task Participants performed an event-related gambling task in which they could decide to either gamble or pass in order to earn as many points as possible 
(Keulers et al., 2011; Stiers et al., 2010). The age-related differences in brain activation during this gambling task are described elsewhere (Keulers et al., 2011). Here, the gambling data will only be used to test the functional specificity of the multivariate maturation patterns obtained from the gonogo data. In each trial of the gambling task a horizontal bar divided into two colored parts was presented (range from 5\%-95\% to $50 \%-50 \%$ ), indicating the probability of an imaginary token being hidden underneath. Participants could guess under which part a token was hidden, by pressing the corresponding left or right button. Depending on the correctness of their choice, points could be won or lost. The points that could be won were indicated above the bar (range 10-100). The points that could be lost were presented below the bar (range 0-100), with the most ambiguous proportions $(50 \%-50 \%)$ coupled with the highest losses $(80-100$ points). Participants could also choose to pass by withholding their response, which resulted consequently in receiving a small 20 points reward. Four trial types along two dimensions could be distinguished: 1) task difficulty: Exogenous versus Endogenous trials depending on whether stimulus characteristics suggested the appropriate choice or not, and 2) task response: Gambling versus Passing trials depending on whether a button was pressed or not. All participants started with 100 points. Feedback about the trial and an update of their total score was provided in $67 \%$ of the trials, in order to disentangle the effects related to feedback processing from those related to the decision making process.

Behavioural analyses The statistical package SPSS 16.0 was used for the analyses. Developmental differences in speed (i.e. mean reaction time), intra- individual speed variability (i.e. standard deviation of reaction time/ mean reaction time (Stuss et al., 2003)) and accuracy (i.e. percentage of false alarms and misses) on the gonogo task were examined. Regarding the mean and variability of reaction times, two analyses of variance (ANOVAs) were conducted with age group (3 levels) as between-subject variable and trial type ( 2 levels: Go vs Go oddball) as within-subject variable. The reaction time data did not violate the parametric assumption of ANOVA. The number of false alarms (i.e. failure to withhold response on Nogo trials) and misses (i.e. failure to react on Go and Go oddball trials) was analyzed with a non- parametric Kruskal-Wallis test due to non-normality. If statistically significant effects were found, post hoc comparisons were performed. The alpha value was set at .05.

Functional magnetic resonance imaging data Image acquisition A Siemens MAGNETOM Allegra 3 T MRI head-only scanner was employed. Head motion was constrained by the use of foam padding. A total number of 32 axial slices covering the whole brain including the cerebellum were imaged by using a T2*-weighted gradient echo planner pulse sequence $(\mathrm{TR}=2000 \mathrm{~ms}, \mathrm{TE}=30 \mathrm{~ms}, \mathrm{FA}=90, \mathrm{FOV}=224$, slice thickness $=4 \mathrm{~mm}$, matrix size $=64 \times 64$, flip angle $=90^{\circ}$ ). Voxel size was $3.5 \times 3.5 \times 4 \mathrm{~mm}$. A T1-weighted anatomical scan was acquired to aid with spatial normalization $\left(\mathrm{TR}=2250 \mathrm{~ms}, \mathrm{TE}=2.6 \mathrm{~ms}\right.$, flip angle $=9^{\circ}, \mathrm{FOV}=256 \mathrm{~mm}$, slice thickness $=1 \mathrm{~mm}$, matrix size $=256 \times 256$, number of slices $=192$ ). Voxel size was $1 \times 1 \times 1 \mathrm{~mm}$. Slice scanning order was ascending interleaved.

Preprocessing Data were preprocessed using BrainVoyager QX, version 1.9 (Brain Innovation, Maastricht, The Netherlands). Images were corrected for slice scan time differences, followed by rigid body motion correction, high-pass temporal filtering, and spatial data smoothing using a Gaussian kernel with a $4 \mathrm{~mm}$ full width at half maximum. 


\section{Chapter 5}

Functional data were coregistered with the anatomical volume using rigid body transformation and manual adjustments to obtain optimal fit by visual inspection if necessary. Subsequently, both functional and structural volumes were transformed into standard stereotaxic space using Talairach normalization. Functional data were resliced to $3 \mathrm{~mm}$ isotropic voxels.

The degree of head motion during scanning was greater in the 13 year-olds compared with the two older age groups on the two most affected parameters (Mayer et al., 2007; Yoo et al., 2005), viz translation in the $\mathrm{z}$ direction $(\mathrm{Tz} ; \mathrm{F}(2,61)=5.89 ; \mathrm{p}=.005)$ and rotation in the $\mathrm{x}$ direction $(\mathrm{Rx} ; \mathrm{F}(2,61)=6.62 ; \mathrm{p}=.002)$. In order to control for the confounding effect of head motion we applied 3 corrections to our data. Firstly, head motion within one scan may distort the measured fMRI signal by spatial misallocation. Therefore, we identified scans during which head motion exceeded a particular threshold, viz absolute motion difference between 2 successive scans in z-direction greater than $0.4 \mathrm{~mm}(1 / 10$ th of the voxel size), and rotation in the $\mathrm{x}$ dimension greater than $0.26^{\circ}$ (angle corresponding to $0.4 \mathrm{~mm}$ z-displacement of frontopolar voxels, assuming the rotation point in middle of brain is $88 \mathrm{~mm}$ from the anterior end of the brain frontal pole (Talairach and Tournoux, 1988)). Task trials (taking into account the hemodynamic response function delay) cause signal intensity changes with peak BOLD values 5 to $6 \mathrm{~s}$ after their occurrence. These signal changes may overlap in time with head motion a few scans later. Consequently, these signal changes are contaminated with within-scan head motion. Therefore, trials in a preceding time window of 1 to $8 \mathrm{~s}$ of the identified scan were modelled as an additional event of no interest. Because this procedure reduced the number of trials available in the events of interest, we set an upper limit of $25 \%$ to the number of trials discarded in this way. As a consequence, 1 young adolescent and 1 old adolescent were excluded because they exceeded this upper limit. After applying this procedure, age groups still differed on the motion parameters for the remaining scans, viz $\operatorname{Tz}(\mathrm{F}(2,59)=6.94 ; \mathrm{p}=.003)$ and $\operatorname{Rx}(\mathrm{F}(2,59)=5.70 ; \mathrm{p}=.005)$. Therefore, as a second measure to equate groups on the motion parameters it was unavoidable to exclude an additional 5 young adolescents with the largest head motion on the $\mathrm{Tz}$ and $\mathrm{Rx}$ parameters. This was effective in eliminating head motion differences between the 3 age groups, viz $\mathrm{Tz}(\mathrm{F}(2,54)=2.53 ; \mathrm{p}=.089)$ and $\mathrm{Rx}(\mathrm{F}(2,54)=2.87$; $\mathrm{p}=.065)$. Thirdly, head motion between successive scans causes signal intensity changes due to inhomogeneities in the magnetic field which are unrelated to the task (Friston et al., 1996). Therefore, we modelled this source of signal variance by included the 6 motion parameters as covariates of no interest in our general linear model (Friston et al., 1996; Johnstone et al., 2006).

Imaging analyses An overview of the image analyses steps and the data flow is provided in Fig. 1. Statistical analyses were performed on individual participant's data by using the general linear model in SPM5 (Welcome Department of Cognitive Neurology, London, UK). The fMRI single subject time series data were modelled as series of events convolved with a canonical hemodynamic response function. A design matrix was set up to model all task conditions of interest (Go, Go oddball and Nogo trials) and the 6 motion parameters. Error trials and trials contaminated with head motion were modelled as two separate predictors that were ignored in the further analyses. Individual percent signal change maps were created for each condition of interest, based on the estimated weights for each of the conditions. These signal change maps were used as the raw data for a second level univariate analysis with subjects as a random effect and for the multivariate pattern classification analysis. 
Univariate analysis A whole brain, voxel-wise general linear model (GLM) with task condition (3 levels) as within-subject variable and age group (3 levels) as betweensubject variable was performed. We identified brain areas underlying the execution of the gonogo task in a contrast that pooled over task conditions and age groups as well as the inhibition contrast Nogo $>$ Go oddball, at the Family Wise Error corrected level (FEW .05) combined with a cluster threshold of 5 voxels. Additionally, we examined age group differences using the univariate voxel-wise GLM. In order to make the GLM results directly comparable with the MVP results, we examined developmental effects for each task condition and each pair-wise age group combination separately (i.e. Go trials 13 vs. 17 year-olds; Go trials 13 vs. 21 year-olds; Go trials 17 vs. 21 year-olds; Go oddball trials 13 vs. 17 year-olds; Go oddball trials 13 vs. 21 year-olds; Go odd- ball trials 17 vs. 21 year-olds; Nogo trials 13 vs. 17 year-olds; Nogo trials 13 vs. 21 yearolds; Nogo trials 17 vs. 21 year-olds). Results are evaluated at the False Discovery Rate (FDR .05) error corrected level, which is a less stringent whole brain correction for multiple comparisons (Genovese et al., 2002). In addition, only activation clusters of 5 or more voxels were considered.

Multivariate pattern classification between age groups We applied MVP classification analysis to the percent signal change maps from the first level analysis in order to examine differences between pair-wise age groups, for each gonogo condition. A whole brain approach was applied, in which all gray matter voxels were examined simultaneously. The analysis was confined to GM voxels to constrain the number of dimensions for computational reasons. Gray matter voxels were selected across participants within each pair-wise age group comparison, first by creating an individual spatially smoothed gray matter mask (Gaussian kernel with $9 \mathrm{~mm}$ full width at half maximum), and second by finding the common voxels across these individual masks that had a gray matter density of at least 0.2 in all masks. The voxels included in the analysis are referred to as features. For each participant, the values of the included voxels in the percent signal change map were converted to a vector/exemplar and labelled according to the age group a participant belonged to. For the MVP classification analysis a version of Support Vector Machines called least squares Support Vector Machines (Suykens et al., 2002) was used and a linear kernel was adopted. This multivariate classification method was combined with recursive feature elimination (RFE) (De Martino et al., 2008; Formisano et al., 2008; Guyon et al., 2002; Staeren et al., 2009). This strategy belongs to the so-called wrapper methods, in which the classifier itself is used to select features that appear to be relevant for the classification at hand. First, for each gonogo task condition and each pair-wise age group combination the dataset was split into $\mathrm{N}$ folds ( $\mathrm{N}=10$ for comparisons between 13 and 17 year-olds, $\mathrm{N}=9$ for 13 versus 21 year-olds, and $\mathrm{N}=19$ for 17 versus 21 year-olds). A cross- validation approach was adopted leaving out data from one fold, which were used as the test set. The remaining data were used as training samples and were further divided in $S$ overlapping splits $(S=3$ for comparisons between 13 and 17 year-olds, $S=4$ for 13 versus 21 year-olds, and $S=3$ for 17 versus 21 year-olds). The classifier was trained separately in each of these splits in order to establish a mathematical function that best discriminates between the age groups. Absolute weights were calculated for each feature/voxel, which coded for the importance of each voxel to the classification. Subsequently, the average absolute weight across the splits was computed. Classification accuracy for this iteration was assessed on the independent test set, not used in the training. Iteratively, the process of training and testing the classifier was 


\section{Chapter 5}

repeated 15 times, i.e. 15 iterations. After each iteration the $30 \%$ features/voxels with the smallest weights were discarded, after which the remaining features/ voxels were used as training samples in the next iteration. This sequence of training and testing over splits and iterations was repeated $\mathrm{N}$ times, each time leaving out data from a different fold as a test set. This cross-validation strategy ensures that the classification result is independent of the choice of training and test set. To arrive at a single accuracy result to report, we first averaged accuracies over the $\mathrm{N}$ folds for each of the 15 iterations. Secondly, to arrive at a final "best iteration" we selected the iteration with the highest classification accuracy. Selecting this iteration is just by way of summary, without intending to imply that it is the only iteration that allows above chance classification, or that it is a global maximum. The $\mathrm{N}$ weight vectors obtained at this best iteration were mapped to the 3D space and averaged as well. In this way a single discriminative map was obtained with voxels that appear most (consistently) discriminative at the majority of the $\mathrm{N}$ folds, having the highest weights. In visualizations of the results only the $30 \%$ highest weighted voxels in a mean weight map are shown. To evaluate the significance of the acquired accuracy values from the described cross-validation, we performed randomization statistics with 1000 permutations and cross-validation (Mourao-Miranda et al., 2005). In each permutation, the analysis was performed as before, but now the age group class labels were randomly assigned to the vectors. Accuracies from these permutations that exceeded or were equal to the accuracy obtained from the best iteration of the original analysis were counted, and their number was converted to a pvalue by adding 1 and dividing by 1000 .

Because parameters in the MVP classification procedure have an effect on the results obtained, we performed the MVP analysis again with different settings for initial and subsequent iterative feature selection in case of insignificant results. Initially, we used multivariate feature selection by discarding the $30 \%$ least contributing voxels at each iteration (total number of iterations $=15$ ), whereas there was no initial voxel selection. It has been shown, however, that initial univariate reduction of the number of voxels can significantly improve the performance of the recursive feature elimination procedure (e.g. De Martino et al., 2008; Ryali et al., 2010). To this end, we repeated the classification analysis with the following settings: 50 iterations and discarding the $10 \%$ least contributing voxels on each iteration. Moreover, we performed an initial voxel selection based on the consistency of responsiveness of the voxels in the current training set (De Martino et al., 2008). Since the optimal number of voxels for initial reduction is not known in advance, we established the optimal number empirically. We ran a crossvalidation within the training set of each fold and thus the estimation was independent from the test. This consists of repeating the same classification with a different number of voxels ranging from the 4000 to the 8000 univariate most responsive voxels, in steps of 2000 voxels. The voxel number yielding the highest accuracy was selected for each task condition, and subsequently the algorithm was run with this optimal amount of initial voxels.

Lastly, all the MVP classification analyses described above were repeated while using a different classifier, namely the Support Vector Machine as implemented by the Spider machine learning toolbox (www.kyb.mpg.de/bs/people/spider/main.html) to make sure that the results were not dependent on the classifier used. Since this yielded comparable accuracies as well as discriminative maps as the initially used classifier, these results are not discussed in the present paper. 
Multivariate pattern generalization across cognitive domains To examine whether functional brain maturation is specific to a particular cognitive function or not, we first established the accuracy with which the age discriminative pattern found by training the classifier on the gonogo data, allowed to classify the same subjects while performing a very different cognitive task, i.e. the gambling task (Keulers et al., 2011). For each pairwise age group combination, the percent signal change maps from all three gonogo conditions were used as training set. The percent signal change maps from the four different task conditions within the gambling task were used as test set. Second, we explored the reversed generalization by using the four percent signal change maps from the gambling task as training set, while the three maps from the gonogo task were used as test set. As both tasks included trials in which a motor response was given as opposed to trials in which a response had to be withheld, we performed an additional MVP analysis to make sure that the generalization is not driven by this similarity in task trial types. Therefore, the classifier was trained on the percent signal change maps from both the Go and Go oddball trials of the gonogo task in which a motor response was executed, while the maps of the Passing trials of the gambling task, in which no motor response was given, were used as test set. The reverse analysis, with training on pass trials from the gambling task and testing on the Go and Go oddball trails from the gonogo task, was also performed. In all these analyses the $30 \%$ least contributing features were eliminated at each iteration over 15 iterations. No initial univariate feature reduction was applied.

There were small differences in participants included in either the gonogo or gambling data. Of the current participants included in the gonogo data $(\mathrm{N}=57)$, three 13 year-olds, two 17 year-olds and two 21 year-olds were not included in the gambling data. In addition, two 13 year-olds, two 17 year-olds and two 21 year-olds were included in the gambling but not in the gonogo data, resulting in 56 datasets for the gambling task.

Post hoc Regions Of Interest based age group analysis Since the MVP classification analysis looks for discriminating voxel response patterns between age groups, this approach gives no directional information of age-related differences in brain activation. Therefore, we performed post hoc Regions Of Interest (ROI) based age group analyses to examine the direction as well as the specific pattern of developmental differences between ages 13 and 21 in BOLD response. A selection of regions that contributed to the age group classification in the MVP analyses was picked out. The final ROIs were identified based on the local maximum of a cluster combined with a radius of $6 \mathrm{~mm}$, based on the MVP results for the pooled gonogo conditions between 13 and 21 yearolds. For each ROI, the average percent signal change (PSC) was extracted for each of the 3 gonogo conditions. Only the $20 \%$ voxels with the highest PSC were included to make the amplitude assessments maximally independent of the extent of activation. For each ROI, the PSC was compared between age groups by conducting an ANOVA with task condition (3 levels) as within- subject variable and age group (3 levels) as betweensubject variable. The assumption of equal error variances across groups (Levene's test) was met for all analyses. Alpha was set at .05 for each ANOVA to give maximal sensitivity. If statistically significant effects were found, post hoc comparisons were performed.

Low frequency functional connectivity analysis Functional connectivity (FC) strength was investigated on the low frequency aspect of the fMRI data collected during performance of the gonogo task. Task- induced modulations of the signal were removed by regressing out the signal variance associated with the task-related GLM model (Fair 


\section{Chapter 5}

et al., 2007b; Fox et al., 2006b). The residual time courses were further preprocessed for FC analysis by detrending (whitening filter implemented in SPM5), regressing out averaged white matter and cerebrospinal fluid signals and temporal band-pass filtering $(0.01-0.1 \mathrm{~Hz})$. We calculated correlation coefficients between each voxels time series and the time series of all other voxels in the gray matter mask already used for selecting MVP features, yielding a voxel by voxel correlation matrix for each participant. The Fischer $\mathrm{z}$ transformation was applied to the correlation coefficients to improve normality. To take into account the spatial scale of connectivity we computed the Euclidean distance between the voxels and categorized voxel pairs in short distance bins of $0-30 \mathrm{~mm}, 30-60 \mathrm{~mm}$ and long distance bins of $60-90 \mathrm{~mm}, 90-120 \mathrm{~mm}$ and beyond $120 \mathrm{~mm}$ (Fair et al., 2007a; Kelly et al., 2009). Subsequently, the mean strength of functional connectivity (Cole et al., 2010; Sepulcre et al., 2010) was calculated within each distance class by averaging the positive correlation coefficients between voxel pairs in each distance bin.

Firstly, we examined whether age-related differences in FC were different for voxels that did or did not contribute to age group discrimination as revealed by the MVP analyses based on task activation. To select voxels for functional connectivity strength analysis, we created a mask that included the $30 \%$ most discriminating voxels for all pair-wise age group combinations as well as for all gonogo conditions as revealed by the MVP analyses. This yielded a contributing mask of 1443 voxels. In addition, we created a non-contributing mask by selected 1443 voxels randomly from the gray matter mask, excluding any voxels in the contributing mask. We quantified the mean strength of functional connectivity of the voxels within each mask located at a distance between 0-30 $\mathrm{mm}, 30-60 \mathrm{~mm}, 60-90 \mathrm{~mm}, 90-120 \mathrm{~mm}$ and more than $120 \mathrm{~mm}$ away, as described above. To examine age differences in FC strength between the voxels in the contributing and non-contributing mask, we performed a repeated measures ANOVA with distance ranges (5 levels) and mask (2 levels) as within subject factors and age group (3 levels) as between subject factor. If statistically significant effects were found, post hoc comparisons were performed. Additionally, polynomial trend analyses were performed for the mean strength of each mask and each distance bin to determine whether the developmental patterns showed a linear and/or a quadratic course.

Secondly, we applied MVP classification analysis to the whole brain connectivity strength maps in order to examine differences between 13 versus 17 year-olds and 17 versus 21 year-olds. For this analysis all gray matter voxels were selected. The whole brain voxel by voxel correlation matrix was divided in only two distance bins, short (0$60 \mathrm{~mm}$ ) and long (>60 mm) ranges, and for each voxel its mean connectivity with short range and long range other voxels was computed. For each participant, the whole brain functional connectivity strength maps for short and long range distances respectively were converted to vectors and labelled according to the age group of a participant. The same cross-validation and selection parameters were used as described for the MVP classification analysis: recursive feature elimination over 15 iterations, discarding the $30 \%$ least contributing voxels, and without initial univariate feature elimination. The classification performance reported is that of the iteration with the highest classification accuracy and the corresponding mean discriminative map, separately for each pair-wise age group combination as well as separately for short and long ranges. 


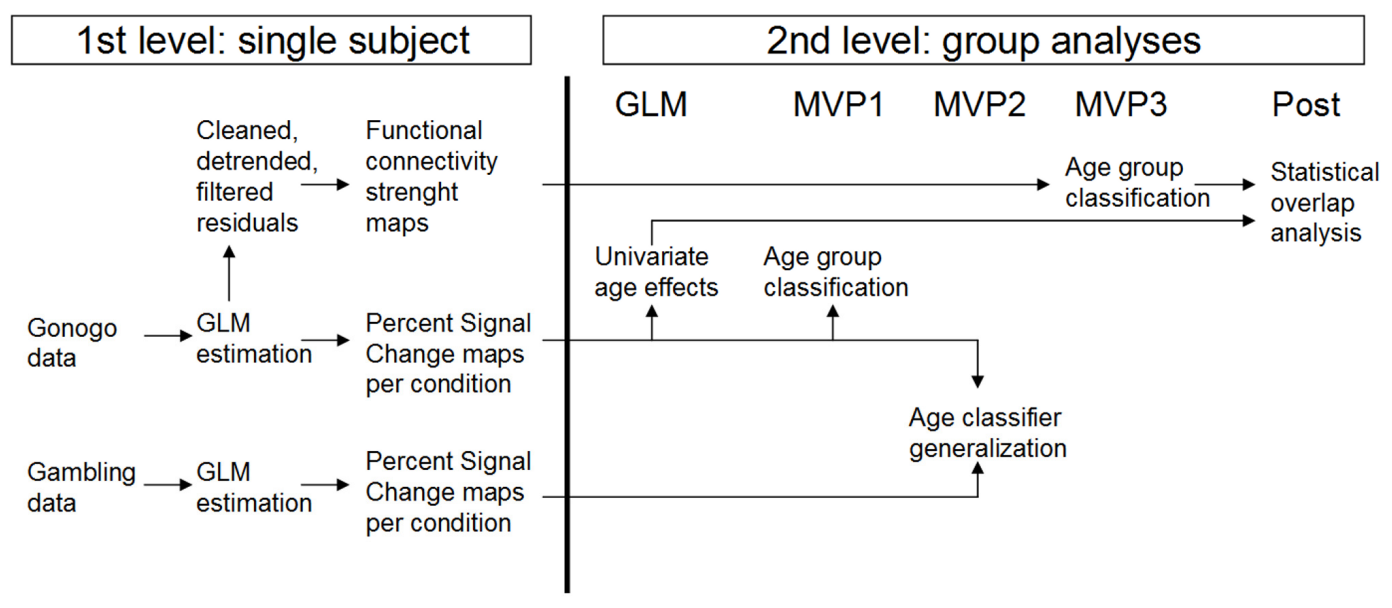

Figure 1. Overview of image analysis steps. At the first level of analysis (left side of figure) preprocessed gonogo data of a single participant are statistically analyzed with general linear model estimation. This estimation is the starting point both for creating functional activation maps and for generating low frequency time series data for functional connectivity analysis. The latter are generated from the GLM residuals by regressing out white matter and CSF signal and band-pass filtering $(0.01-0.1 \mathrm{~Hz})$ (upper line of figure). The functional activation maps are generated as condition specific percent signal change maps from the estimated betas (middle line of figure). Similar percent signal change maps were available from a previous study (Keulers et al., 2011) for the conditions of a gambling task (lower line in figure). At the second level (right side of figure) the single participant data were integrated into several group level analyses. First, a univariate estimation of age effects was performed on the gonogo percent signal change maps (GLM; see Univariate analysis and Task and developmental effects revealed by the univariate voxel-wise analysis sections). These age effects were also estimated in a multivariate pattern analysis from the same percent signal change maps (MVP1; see Multivariate pattern classification between age groups, Age classification performance and Pattern of brain areas contributing to age group classification sections). In addition, the gonogo percent signal change maps are used together with the gambling percent signal change map in a multivariate pattern analysis as a test of the specificity of age discriminating patterns (MVP2; see Multivariate pattern generalization across cognitive domains and Generalizability of age-distinctive functional patterns sections). A third multivariate pattern analysis was performed on the functional connectivity strength maps, also to look for age effects (MVP3; see Low frequency functional connectivity analysis and Relation to developmental effects in low frequency functional connectivity sections). Lastly, a post hoc analysis of the overlap in the weight maps resulting from MVP1 and MVP3 was performed (see Relation to developmental effects in low frequency functional connectivity section).

\section{$\underline{\text { Results }}$}

Behavioural performance The behavioural results showed an overall decrease in mean reaction time with age $(\mathrm{F}(2,54)=13.13 ; \mathrm{p}<.001)$. The 13 year-olds reacted slower compared with $17(\mathrm{p}=.022)$ and $21(\mathrm{p}<.001)$ year-olds, and 17 year-olds in their part were slower than 21 year-olds $(\mathrm{p}=.043)$. In addition, the ANOVA showed a main effect of trial type with longer reaction times on Go oddball compared with Go trials ( $\mathrm{F}(2$, $54)=14.25 ; \mathrm{pb} .001)$. This suggests that reacting on less frequent Go odd- ball trials is more difficult compared with frequent Go trials. There was no significant interaction between age group and trial type.

Regarding the speed variability, the ANOVA revealed a main effect for age group $(F(2,54)=15.15 ; \mathrm{pb} .001)$ and an age group $\times$ trial type interaction effect $(\mathrm{F}(2,54)=3.67$; $\mathrm{p}=.032)$. On Go trials the 13 year-olds showed more variability in reaction times than 17 $(\mathrm{p}<.001)$ and $21(\mathrm{p}<.001)$ year-olds, whereas on Go oddball trials both 13 and 17 year- 


\section{Chapter 5}

olds showed more speed variability compared with 21 year- olds (respectively $\mathrm{p}<.001$ and $\mathrm{p}=.004)$.

Task and developmental effects revealed by the univariate voxel-wise analysis Across age groups, positive responding areas were found during performance of the gonogo task in the anterior cingulate cortex, anterior insula, inferior frontal sulcus, subcortical and occipital regions, which have been described as part of a large task-related network (e.g. Dosenbach et al., 2007; Sridharan et al., 2008; Stiers et al., 2010). In contrast, deactivation during task execution was observed in several areas, including the posterior cingulate cortex, superior temporal and parahippocampal gyri, among others, which have been described as part of the default mode network (e.g. Dosenbach et al., 2007; Greicius et al., 2003; Stiers et al., 2010). Task related activations and deactivations are visualized in Fig. 2 (white and dark gray clusters, respectively). A list of all significant activation clusters during general gonogo performance is provided in Supplementary Table 1. With respect to inhibition specific activation, we found significant clusters in the bilateral insula (Talairach coordinates: 30, 15, 3 and -36, 21, 3), dorsal part of the anterior cingulate cortex (Talairach coordinates: 0, 12, 42 and 0, 30, 21), and right inferior part of the precentral sulcus (Talairach coordinates: 39, -6, 45) (contrast Nogo $>$ Go oddball; FWE $<.05 ; \mathrm{T}=4.81$; see Fig. 2, yellow clusters). These findings confirm that our gonogo task was effective in modulating the BOLD signal in distributed functional brain networks. The identified task activated and deactivated clusters, which will be referred to as the "task positive" and "task negative" areas or regions, will be used as a reference frame to interpret developmental differences in brain activation.

The univariate GLM analysis revealed only a small number of voxel clusters where brain activation differed at the FDR error corrected significance level between age groups during the execution of the gonogo task (Table 1). The largest effect was found between 13 and 21 year-olds, in all task conditions, with the largest difference in the go condition comprising 195 voxels divided over 11 clusters. For the other age group comparisons, there was only one cluster that significantly differed between 13 and 17 year-olds (6 voxels in the go condition), and one between 17 and 21 year-olds (26 voxels, also in the go condition). Of the 22 voxel clusters that showed an effect in any of the comparisons, the majority fell within or partially overlapped (i.e. located at the border) with the earlier identified task positive and task negative responding areas. Only 4 age-related clusters did not overlap with task related areas (see Table 1, last column). To further explore the univariate effects of age on brain activation, we visualized in Fig. 2 the age group related statistical maps, thresholded for improved visualization at the uncorrected significance level of $\mathrm{p}=.001$. This lower threshold yielded for each task condition and pair-wise age group combination only a few, small additional clusters.

Because age groups also differed in their performance on the gonogo task we repeated the above analyses with the relevant performance parameters (mean reaction time, speed variability and number of misses) as covariates. This yielded nearly identical results. In fact, the statistical maps depicting brain activity across age groups that were associated with task performance covariates revealed no significant voxel clusters at the FDR error corrected significance level. 


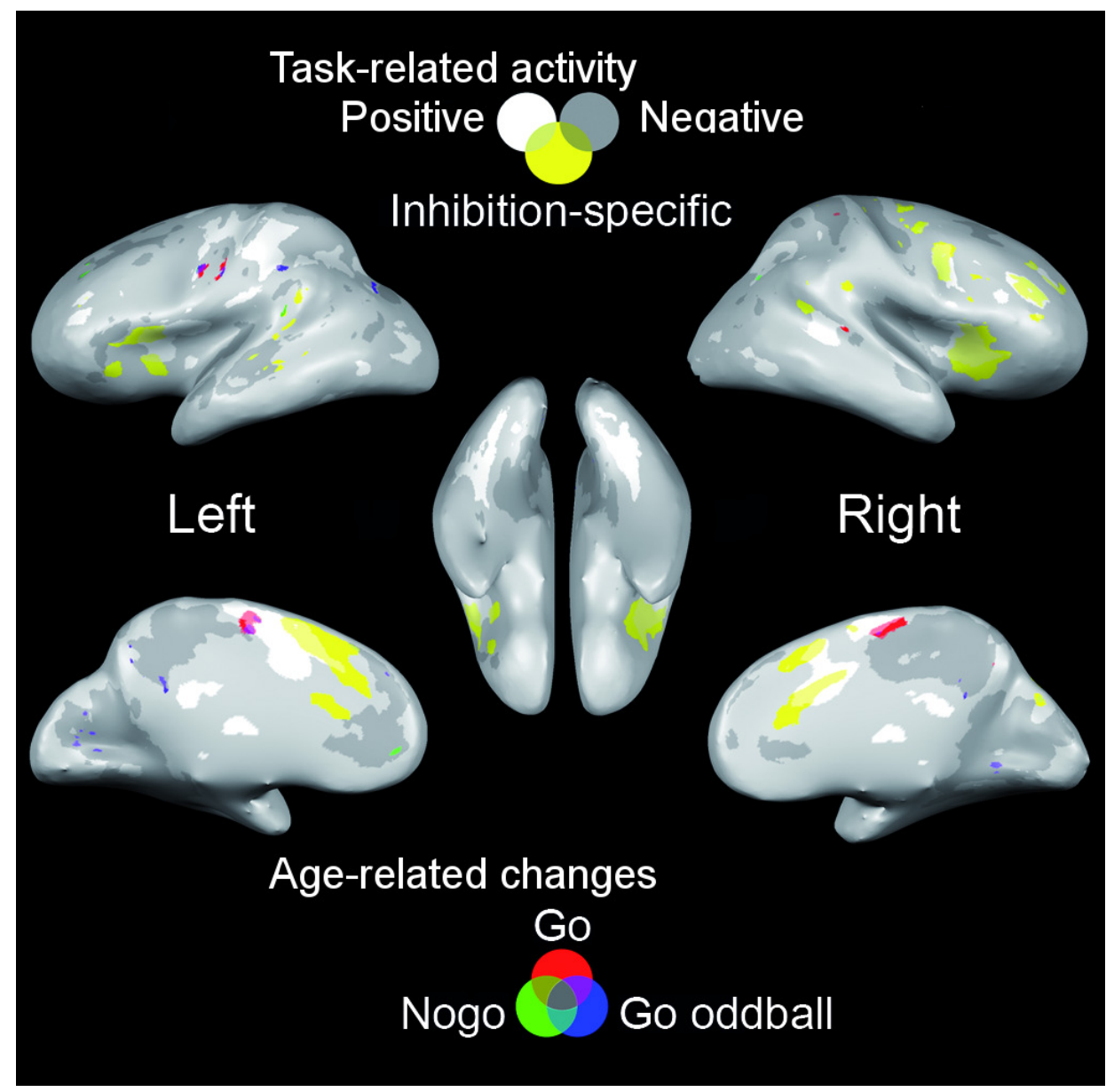

Figure 2. Differences in brain activation between 13 and 21 year-olds during gonogo performance revealed by the univariate voxel-wise analysis. Age group differences are presented separately for each task condition: Go (red), Go oddball (blue) and Nogo (green) trials. F-Maps are thresholded at the less stringent uncorrected significance level of $\mathrm{p}=.001(\mathrm{~F}=11.23)$ for the sake of visualization. Age-related differences are overlaid on the age-independent task-responsive activations thresholded at the False Discovery Rate (FDR) corrected significance level: task positive responding areas (white, $\mathrm{T}=2.44$ ), task negative responding areas (dark gray, $\mathrm{T}=2.23$ ), and inhibition specific areas (Nogo $>$ Go oddball, yellow, $\mathrm{T}=2.84$ ) are visualized.

Developmental effects revealed by the multivariate pattern classification analysis

The MVP classification analysis was applied to the percent signal change maps from the first level analysis in order to examine differences between pair-wise age groups, for each gonogo task condition.

Age classification performance Classification accuracies per iteration for each task condition and pair-wise age group combination are shown in Fig. 3, and the best iteration results in Table 2. The highest accuracies were obtained in dis- criminating 13 from 21 year-olds, for all task conditions (Go: 80.8\%; Go oddball: 80.1\%; Nogo: $84.0 \%$ ). Of 1000 random permutations only $0.1-0.3 \%$ resulted in higher accuracy values than the observed ones. The actual classification results can therefore be considered statistically highly significant (Go: $\mathrm{p}=.001$; Go oddball: $\mathrm{p}=.003$; Nogo: $\mathrm{p}=.001)$. Categorizing 13 against 17 year-olds was correct in $77.5 \%(p=.004)$ based on 


\section{Chapter 5}

signal changes during Go trials, in $68.9 \%(\mathrm{p}=.031)$ during Go oddball trials and in $70.0 \%(p=.020)$ during Nogo trials. Because initially non-significant accuracies were observed in discriminating between 17 and 21 year-olds (Go: 62.3\%, p>.1; Go oddball: $58.8 \%, \mathrm{p}>.1$; Nogo: $50.9 \%, \mathrm{p}>.1$ ), we repeated the classification analysis with different settings for initial and iterative feature selection (see Multivariate pattern classification between age groups section and Table 2). With these new settings, accuracies reached a statistical significant level also for the 17 versus 21 year-olds classification, with the highest accuracies emerging only in the last iteration steps, i.e. step 49 or 50 (Go: 70.2\%, $\mathrm{p}=.023$; Go oddball: 72.8\%, $\mathrm{p}=.019$; Nogo: 67.5\%, $\mathrm{p}=.055$ ). These results suggest that the functional maturation pattern between 17 and 21 year-olds is confined to smaller numbers of voxels, making the analysis more susceptible to non-age related and/or noise patterns in the data (De Martino et al., 2008). When these nondiscriminating voxels are eliminated by the initial feature reduction step, classification accuracy increases to the range obtained for the other age comparisons.

We performed a control analysis to investigate the possible effect of task performance differences between age groups on the MVP results. For this purpose, we used the model estimates from the GLM analysis that included the three performance measures as covariates (see Task and developmental effects revealed by the univariate voxel-wise analysis section) to remove the estimated covariance effects across participants. The classification accuracies obtained with these cleaned data were overall higher than with the original data, with accuracies for different age group and gonogo condition comparisons ranging from 72 to $97 \%$. The better results after cleaning the data indicate that task performance related BOLD signal differences constitute a meaningful activation pattern that interferes with age group classification. Despite the improved accuracies, we will report the results from the uncleaned data only, to preserve the continuity with the generalization analyses (see Multivariate pattern generalization across cognitive domains section), where we compare gonogo data with data from the gambling task in which there was no performance difference between age groups.

Pattern of brain areas contributing to age group classification The discriminative weight maps showing the spatial pattern by which age groups significantly differ for each task condition are shown in Fig. 4. Voxel clusters collectively contributing to age group classification were distributed throughout the whole brain, rather than being confined to one or more individual regions. Additionally, a significant portion of the contributing voxels overlapped with either task positive or task negative responding areas, i.e., $51.1 \%$ in 13 versus 17 year-olds $\left(\chi^{2}=67.9, \mathrm{p}<.001\right)$ and $55.6 \%$ in 17 versus 21 year-olds $\left(\chi^{2}=6.5, p<.01\right)$. Firstly, some of the brain regions that contributed to age group classification are associated with the default mode network, as they fall within or partially overlap with task-induced deactivated areas (Fig. 4). These are evident in ventral medial prefrontal cortex, posterior cingulate cortex and anterior calcarine sulcus. In addition, discriminating regions were found in bilateral temporal and parahippocampal gyri, areas that also have been included in the default mode network (Beckmann et al., 2005; Dosenbach et al., 2007; Greicius et al., 2003). Secondly, several contributing regions fall within or partially overlap with activated areas during execution of the gonogo task (Fig. 4). For both the go and go oddball conditions, discriminating clusters emerged in supplementary motor area, precentral and postcentral sulci, and posterior insula, all of which are included in the sensory-motor network as described in resting state imaging studies (Beckmann et al., 2005; Damoiseaux et al., 2006; De Luca et al., 2006). In addition, voxels in attention related areas of the right 
intraparietal sulcus and frontal eye field (Corbetta and Shulman, 2002; Fox et al., 2006a) contributed to age discrimination. In the visual processing network (Beckmann et al., 2005; Damoiseaux et al., 2006; De Luca et al., 2006), areas in bilateral fusiform and lingual gyri contributed to age group classification. Furthermore, voxel clusters in areas of the prefrontal cortex associated with salience and central executive networks (Dosenbach et al., 2007; Sridharan et al., 2008) were found to contribute to age group discrimination.

Table 1. Results of the general linear model pair-wise age group comparisons for each gonogo condition compared to rest

\begin{tabular}{|c|c|c|c|c|c|c|c|}
\hline $\begin{array}{l}\text { Task } \\
\text { condition }\end{array}$ & $\begin{array}{l}\text { Age group } \\
\text { comparison }\end{array}$ & Brain area & $\begin{array}{l}\text { TAL } \\
\begin{array}{ll}x \quad y \quad z\end{array}\end{array}$ & Size & $\begin{array}{l}\text { F- } \\
\text { value }\end{array}$ & $\begin{array}{l}\mathrm{P}_{\mathrm{FDR}} \\
\text { corrected }\end{array}$ & $\begin{array}{l}\text { Task } \\
\text { pos/ } \\
\text { neg }\end{array}$ \\
\hline \multirow[t]{3}{*}{ Go } & 13 vs 17 & 1 precentral sulcus & $-36-2451$ & 6 & 23.96 & .042 & pos \\
\hline & 13 vs 21 & $\begin{array}{l}\text { r STG } \\
\text { m SMA } \\
\text { r cerebellum culmen } \\
1 \text { inf. precentral sulcus } \\
\text { r thalamus VMP } \\
\text { r postcentral sulcus } \\
\text { l parahip. gyrus } \\
\text { l postcentral sulcus } \\
1 \text { cerebellum culmen } \\
1 \text { trans. temp. gyrus } \\
\text { m posterior cingulate }\end{array}$ & $\begin{array}{l}57-3315 \\
0-1557 \\
21-63-24 \\
-51-933 \\
9-183 \\
27-3651 \\
-24-48-3 \\
-57-2436 \\
-36-54-24 \\
-45-3012 \\
-6-4536\end{array}$ & $\begin{array}{l}28 \\
49 \\
34 \\
26 \\
10 \\
7 \\
6 \\
8 \\
9 \\
8 \\
10\end{array}$ & $\begin{array}{l}33.04 \\
28.95 \\
26.70 \\
19.69 \\
18.98 \\
17.39 \\
16.68 \\
16.26 \\
16.02 \\
15.77 \\
15.24\end{array}$ & $\begin{array}{l}.001 \\
.003 \\
.003 \\
.018 \\
.020 \\
.027 \\
.033 \\
.036 \\
.038 \\
.040 \\
.046\end{array}$ & $\begin{array}{l}\text { pos } \\
\text { pos } \\
\text { pos } \\
\text { neg } \\
\text { neg } \\
\text { pos } \\
\text { pos } \\
\text { neg } \\
\text { neg }\end{array}$ \\
\hline & 17 vs 21 & r cerebellum culmen & $21-63-24$ & 26 & 26.99 & .017 & pos \\
\hline Go & 13 vs 17 & no significant clusters & & & & & \\
\hline \multirow[t]{2}{*}{ oddball } & 13 vs 21 & $\begin{array}{l}\text { r lingual gyrus } \\
\mathrm{r} \text { cerebellum }\end{array}$ & $\begin{array}{l}18-510 \\
24-66-24\end{array}$ & $\begin{array}{l}78 \\
12\end{array}$ & $\begin{array}{l}23.13 \\
21.64\end{array}$ & $\begin{array}{l}.048 \\
.048\end{array}$ & $\begin{array}{l}\text { neg } \\
\text { pos }\end{array}$ \\
\hline & 17 vs 21 & no significant clusters & & & & & \\
\hline \multirow[t]{3}{*}{ Nogo } & 13 vs 17 & no significant clusters & & & & & \\
\hline & 13 vs 21 & $\begin{array}{l}\mathrm{r} \text { IPS } \\
1 \text { SFS } \\
1 \text { inf. precentral sulcus } \\
\mathrm{m} \text { ventral PFC } \\
\mathrm{r} \text { inf. precentral } \\
1 \text { STG } \\
\mathrm{r} \text { cuneus }\end{array}$ & $\begin{array}{l}27-6633 \\
-243633 \\
-51-639 \\
3453 \\
39-630 \\
-60-3918 \\
27-7215\end{array}$ & $\begin{array}{l}43 \\
8 \\
9 \\
42 \\
16 \\
6 \\
7\end{array}$ & $\begin{array}{l}25.33 \\
24.20 \\
24.15 \\
23.99 \\
23.27 \\
18.78 \\
17.47\end{array}$ & $\begin{array}{l}.011 \\
.011 \\
.011 \\
.011 \\
.013 \\
.031 \\
.046\end{array}$ & $\begin{array}{l}\text { pos } \\
\text { neg } \\
\text { pos } \\
\text { neg } \\
\text { pos }\end{array}$ \\
\hline & 17 vs 21 & no significant clusters & & & & & \\
\hline
\end{tabular}

Note. ${ }^{\text {a }}$ Indication whether activation clusters fall within and/or overlap with task positive responding areas (=pos), task negative responding areas $(=n e g)$, or do not overlap with task-related areas at all (empty). Size in number of voxels. TAL $=$ Talairach coordinates; $F D R=$ False Discovery Rate error corrected level; $r=$ right hemisphere; $1=$ left hemisphere; $\mathrm{m}=$ medial; $\mathrm{STG}=$ Superior Temporal Gyrus; $\mathrm{SMA}=$ Supplementary Motor Area; inf. $=$ inferior; $\mathrm{VMP}=$ ventral medial nucleus of the thalamus; parahip. $=$ parahippocampal; trans. $=$ transverse; temp. $=$ temporal; IPS $=$ Intraparietal Sulcus; SFS = Superior Frontal Sulcus; PFC $=$ Prefrontal Cortex.

These included the anterior cingulate cortex and anterior insula, although the latter only contributed in the 13 versus 17 year-old comparison. In addition, a cluster was found in the left inferior frontal junction that contributed as well to age group differentiation, 


\section{Chapter 5}

although other areas of the central executive network did not contribute to age group classification. Thirdly, some of the regions contributing to age group discrimination did not overlap with positive or negative task responding areas, namely in the precuneus and left inferior frontal sulcus.

These results show that both task positive and task negative brain networks as well as brain regions not directly implicated in gonogo performance by univariate analysis, collectively differentiate between activation patterns of 13, 17 and 21 year-olds.

Generalizability of age-distinctive functional patterns To investigate the generalizability of the distributed discriminating age patterns over cognitive functions, we established the accuracy with which the pattern found by training the classifier on the current gonogo data, allowed to classify the same participants while performing a very different cognitive task, i.e. a gambling task (Keulers et al., 2011). This analysis established a high generalization ability as the classifier could discriminate with high accuracies between different age groups in the test data: 70.1\% accuracy for 13 versus 17 yearolds (p .05), 78.1\% accuracy for 13 versus 21 year-olds ( $\mathrm{p} .01$ ), and $82.9 \%$ for 17 versus 21 year-olds ( $\mathrm{p} .01$ ). The reversed generalization from gambling to gonogo task was equally successful: $69.9 \%$ accuracy for 13 versus 17 year-olds (p .05), 76.7\% accuracy for 13 versus 21 year-olds (p .01), and $77.0 \%$ for 17 versus 21 year-olds (p .01). The ability to generalize age discriminating activation patterns across different cognitive tasks cannot be explained by simple similarities between these tasks, such as performing a motor response to a stimulus or inhibiting a response set. This is evident from the fact that a classifier trained on Go and Go oddball trials from the gonogo task, in which participants have to give a motor response, is equally able to classify age groups based on activation maps from gambling trials in which no motor response was given: $71.5 \%$ accuracy for 13 versus 17 year-olds ( $\mathrm{p} .05), 79.4 \%$ accuracy for 13 versus 21 year-olds ( $\mathrm{p} .01$ ), and $78.6 \%$ for 17 versus 21 year-olds (p .01). Again, similar results were obtained when switching train and test sets.

These results suggest that developmental maturation in brain functioning constitutes a general pattern of activity change, that is not specific to the particular cognitive task being executed or the specific cognitive processes invoked by such a task. This is because the selection of features/voxels after each training iteration is based only on their contribution to age group differentiation in the training task data. Hence, these differentiating characteristics can be related to neural or cognitive processes that are specific to the demands of the training task as well as to unspecific central processes that are common to both tasks. The fact that the characteristic pattern for the training data was equally distinctive for the test task data indicates that the classification is not dependent on activation that is unique to the training task but mostly depends on activation that the two tasks have in common. 
A) 13 versus 17 year-olds

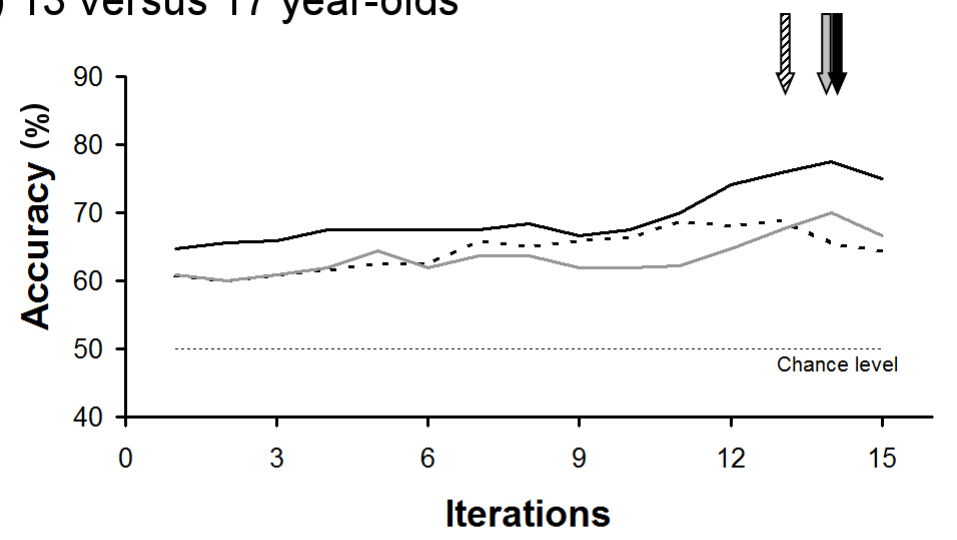

B) 13 versus 21 year-olds

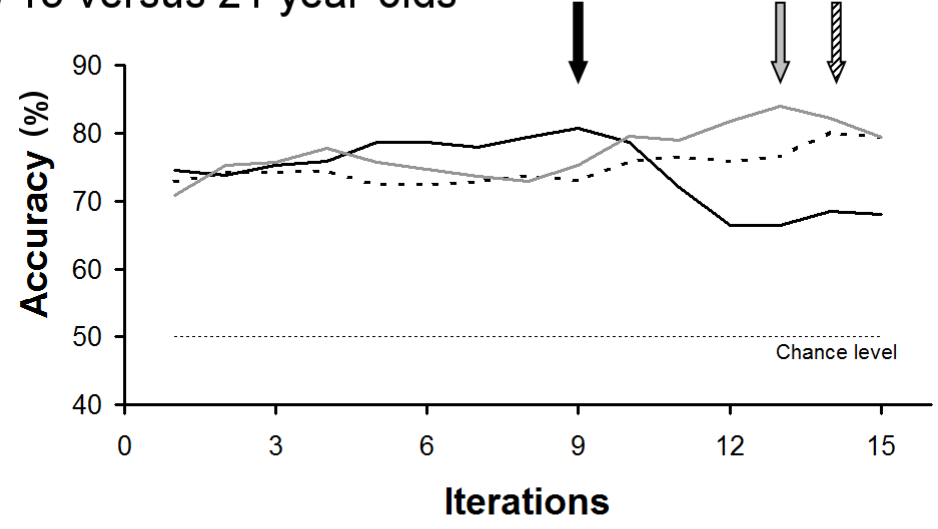

\section{C) 17 versus 21 year-olds}

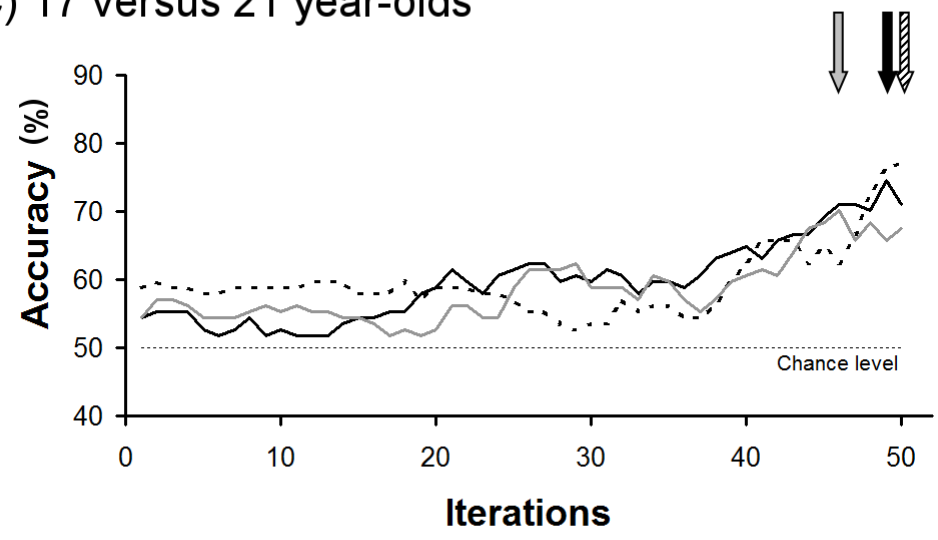

Figure 3. Multivariate pattern classification accuracies per task condition and pair-wise age group comparison in relation to the progress of the recursive feature elimination process. Each graph shows the mean accuracies over the folds on the data set at each iteration of the recursive process for discriminating between respectively 13 and 17 year-olds (A), 13 and 21 year-olds (B), and 17 and 21 year-olds (C). The three lines in each graph represent the classification result for activation maps derived from different task conditions: Go (black), Go oddball (dotted) and Nogo (gray) trials. The horizontal line at 50\% accuracy represents chance level. The arrows indicate the best iteration, i.e., with the highest accuracy over folds, for each task condition. This best iteration accuracy is reported as the classification result in the text and Table 2. 
Chapter 5

Table 2. Multivariate pattern age classification performance at the best iteration, for two sets of feature elimination parameters.

\begin{tabular}{|c|c|c|c|c|}
\hline \multirow{2}{*}{\multicolumn{5}{|c|}{15 iterations, $30 \%$ discarding }} \\
\hline & & & & \\
\hline \multicolumn{2}{|c|}{\begin{tabular}{l|l}
15 iterations, 30\% discarding \\
\end{tabular}} & \multirow{2}{*}{\begin{tabular}{|r}
$13-17 \mathrm{yr}$. \\
77.5
\end{tabular}} & \multirow{2}{*}{\begin{tabular}{|r|}
$13-21 \mathrm{yr}$. \\
80.8
\end{tabular}} & \multirow{2}{*}{$\begin{array}{r}17-21 \mathrm{yr} . \\
62.3\end{array}$} \\
\hline Go & Accuracy (\%) & & & \\
\hline & Randomisation $\mathrm{p}$ & 0.004 & 0.001 & ns. * \\
\hline & Best iteration & 14 & 9 & 11 \\
\hline & & & & \\
\hline Go oddball & Accuracy (\%) & 68.90 & 80.10 & 58.80 \\
\hline & Randomisation $\mathrm{p}$ & 0.031 & 0.003 & ns. \\
\hline & Best iteration & 13 & 14 & 7 \\
\hline & & & & \\
\hline Nogo & Accuracy (\%) & 70.00 & 84.00 & 50.90 \\
\hline & Randomisation $\mathrm{p}$ & 0.020 & 0.001 & ns. \\
\hline & Best iteration & 14 & 13 & 5 \\
\hline \multicolumn{5}{|c|}{50 iterations, $10 \%$ discarding and initial voxel selection } \\
\hline & & $13-17 \mathrm{yr}$. & $13-21 \mathrm{yr}$ & $17-21 \mathrm{yr}$ \\
\hline Go & Accuracy (\%) & 75.0 & 75.5 & 74.6 \\
\hline & Randomisation $\mathrm{p}$ & na.\# & na. & 0.023 \\
\hline & Best iteration & 34 & 18 & 49 \\
\hline & Initial voxels & 4000 & 4000 & 4000 \\
\hline Go oddball & Accuracy (\%) & 71.7 & 97.2 & 77.2 \\
\hline & Randomisation $\mathrm{p}$ & na. & na. & 0.019 \\
\hline & Best iteration & 50 & 49 & 50 \\
\hline & Initial voxels & 4000 & 6000 & 8000 \\
\hline & & & & \\
\hline Nogo & Accuracy (\%) & 69.4 & 81.5 & 70.2 \\
\hline & Randomisation $\mathrm{p}$ & na. & na. & 0.005 \\
\hline & Best iteration & 50 & 40 & 46 \\
\hline & Initial voxels & 4000 & 6000 & 4000 \\
\hline
\end{tabular}

Note.

* ns.=not significant; \# na. =not available.

Post hoc ROI analyses MVP classification analysis does not provide information about the direction of age-related differences in brain activation. To explore the differences underlying age classification we performed post hoc ROI-based age group analyses for a selection of regions that contributed to age group discrimination in the MVP analyses. 
In two task-induced deactivated clusters that contributed to age group discrimination, i.e. ventral-anterior medial prefrontal and posterior cingulate cortex, PSC decreased with age (respectively $F(2,53)=8.05 ; p=.001$ and $F(2,54)=8.40 ; p=.001$ ). The 21 yearolds deactivated these regions more compared with 13 year-olds (respectively $p<.001$ and $\mathrm{p}<.001$ ), with an intermediate position for the 17 year-olds which failed to reach significance (Fig. 5A). From the task positive areas that contributed to age group discrimination, we selected several regions from the different positive networks for ROI analysis (Fig. 5B): viz supplementary motor area, postcentral gyrus, intraparietal sulcus, frontal eye field, anterior cingulate cortex and anterior insula. PSC in the two motor network areas increased with age, viz supplementary motor area $(\mathrm{F}(2,54)=4.56$; $\mathrm{p}=.015)$ and postcentral gyrus $(\mathrm{F}(2,54)=5.97 ; \mathrm{p}=.005)$. Postcentral gyrus was activated more by 21 and 17 year-olds compared with 13 year-olds (respectively $p=.018$ and $\mathrm{p}=.009$ ), whereas only 21 year-olds activated supplementary motor area stronger compared with 13 year-olds $(\mathrm{p}=.021)$, with an intermediate, non- significant position for the 17 year-olds. In the attention-related frontal eye field, the BOLD response increased with age as well $(\mathrm{F}(2,54)=6.07 ; \mathrm{p}=.004)$, with higher PSC for 21 and 17 year-olds compared with 13 year-olds (respectively $p=.009$ and $p=.016$ ). In the second attentionrelated ROI, right intraparietal sulcus, the interaction of age and task condition was significant $(\mathrm{F}(2,54)=6.45 ; \mathrm{p}=.003)$. The age effect was strongest during Nogo trials $(\mathrm{F}(2,54)=9.18 ; \mathrm{pb} .001)$, followed by Go oddball trials $(\mathrm{F}(2,54)=3.94 ; \mathrm{p}=.025)$ and only borderline significant during Go trials $(\mathrm{F}(2,54)=3.01 ; \mathrm{p}=.058)$. Furthermore, enhanced intraparietal activation during Nogo compared with Go trials $(p=.004)$ with an intermediate, non-significant position for Go oddball trails was only seen in 21 yearolds and not in 13 and 17 year-olds. The effect of age group on PSC failed to reach significance in the areas of the salience network, viz anterior cingulate cortex and anterior insula. Thus, task-induced deactivation of default mode areas increased with age. At the same time, activation of motor and attention related areas increased, while there was no difference in typical response control areas. Only in the attention-related right intraparietal sulcus the age effect was modulated by task conditions, with enhanced activation during inhibition only in 21 year-olds.

Relation to developmental effects in low frequency functional connectivity To directly relate our MVP findings on age-related differences in task-induced activation to the development of functional connectivity, we quantified the low frequency functional connectivity strength between individual voxels located at distances between $0-30 \mathrm{~mm}$, $30-60 \mathrm{~mm}, 60-90 \mathrm{~mm}, 90-120 \mathrm{~mm}$, and more than $120 \mathrm{~mm}$ away. First, we examined whether age-related differences in short and long range functional connectivity were different for voxels that did and did not contribute to the age group discrimination as revealed by the MVP based on task activation. Contributing voxels were identified by an image mask that grouped the $30 \%$ most contributing voxels across all MVP comparisons. The non-contributing mask contained a similar number of voxels that were randomly selected from the gray matter mask, excluding all voxels that contributed to task- related age classification. The developmental relationship between functional connectivity and task-related activation was addressed with a three-way ANOVA ( 5 distance ranges $\times 2$ MVP contribution masks $\times 3$ age groups) on the average functional connectivity strength of voxels. The analysis revealed a highly significant interaction between the distance of voxels and whether they contributed to the MVP analysis or not $(\mathrm{F}(2,54)=190.50 ; \mathrm{p}<.001)$. Post-hoc analyses revealed that the mean functional connectivity strength was higher in voxels that contributed to the MVP 


\section{Chapter 5}

classification compared with non contributing voxels for all distances but the shortest $(0-30 \mathrm{~mm})$, which showed the reverse effect (see Fig. 6F). This means that voxels contributing to the maturation of brain activation during task performance have on average stronger long range connections and less short range connections. This is consistent with the location of many of these voxels in the vicinity of the major largescale functional brain networks. The hypothesized interaction of this effect with age (i.e. that the strength of long range connections in the contributing voxels increases with age) was borderline significant $(\mathrm{F}(2,54)=1.95 ; \mathrm{p}=.061)$. Therefore, we repeated the ANOVA for each distance separately. This revealed that the interaction was due to the 0-30mm voxel pairs, for which the higher connectivity in the non-contributing compared with contributing voxels was strongest in 13 year-olds followed by 17 and 21 year-olds $(F(2,54)=3.50 ; p=.038$; see Fig. $6 \mathrm{~A})$. In none of the other distances was a significant age by MVP contribution mask interaction. More sensitive linear trend analyses for each distance did reveal a significant linear increase of connectivity strength in the MVP contributing voxels at the range of $90-120 \mathrm{~mm}(\mathrm{~F}(2,54)=4.07$; $\mathrm{p}=.049$ ). The same effect in the non-contributing voxels was only borderline significant, as was the effect at the $60-90 \mathrm{~mm}$ and $>120 \mathrm{~mm}$ distance for the contributing voxels. This result of a mild increase in long range connectivity with age confirms previous reports (Fair et al., 2007a, 2008, 2009; Kelly et al., 2009). However, as illustrated in Figs. 6B-E this effect was similar in both voxel masks, albeit somewhat less significant in the mask of voxels not contributing to the MVP classification. These results do not support the hypothesis that brain activation maturation is associated with functional connectivity maturation simply by both taking place in the same voxels, at least not if connectivity is considered at the whole brain level. As a second step in the investigation of the relationship between task-induced and low-frequency connectivity maturation, we applied MVP classification analysis directly on the whole brain connectivity strength maps, separately for short $(0-60 \mathrm{~mm})$ and long $(>60 \mathrm{~mm})$ range connections. This yielded significant discrimination accuracies between 13 and 17 year-olds for short $(78.3 \% ; \mathrm{p}<.01)$ and long $(74.4 \% ; \mathrm{p}<.01)$ range connections. In contrast, categorizing 17 against 21 year-olds was significantly above chance only for long range connections $(72.8 \% ; \mathrm{p}<.05)$, while the accuracy obtained at the short range were at chance level (43\%; this remained non-significant for different MVP parameter settings). The three significant discriminative weight maps are visualized in Fig. 7. The weight maps for short range FC are concentrated in somatosensory and motor regions, which preferentially show short range connectivity (Sepulcre et al., 2010). The weight maps only partially overlapped with the weight maps based on task activation, in that the most contributing voxels in the task-related weight map overlapped only $23.8 \%$ with the most contributing voxels in the long range FC weight map for 13 versus 17 year-old comparison $\left(\chi^{2}=1.54, \mathrm{p}=.30\right)$, and $6.9 \%$ in the 17 versus 21 year-old comparison $\left(\chi^{2}=1.9\right.$, $\mathrm{p}=.20$ ). This result again suggests that particularly long range connectivity matures drastically during adolescence, and while this maturation is likely to underlie changes in brain activity in the same period, their distribution does not coincide with the distribution of brain activity maturation. 
Chapter 5

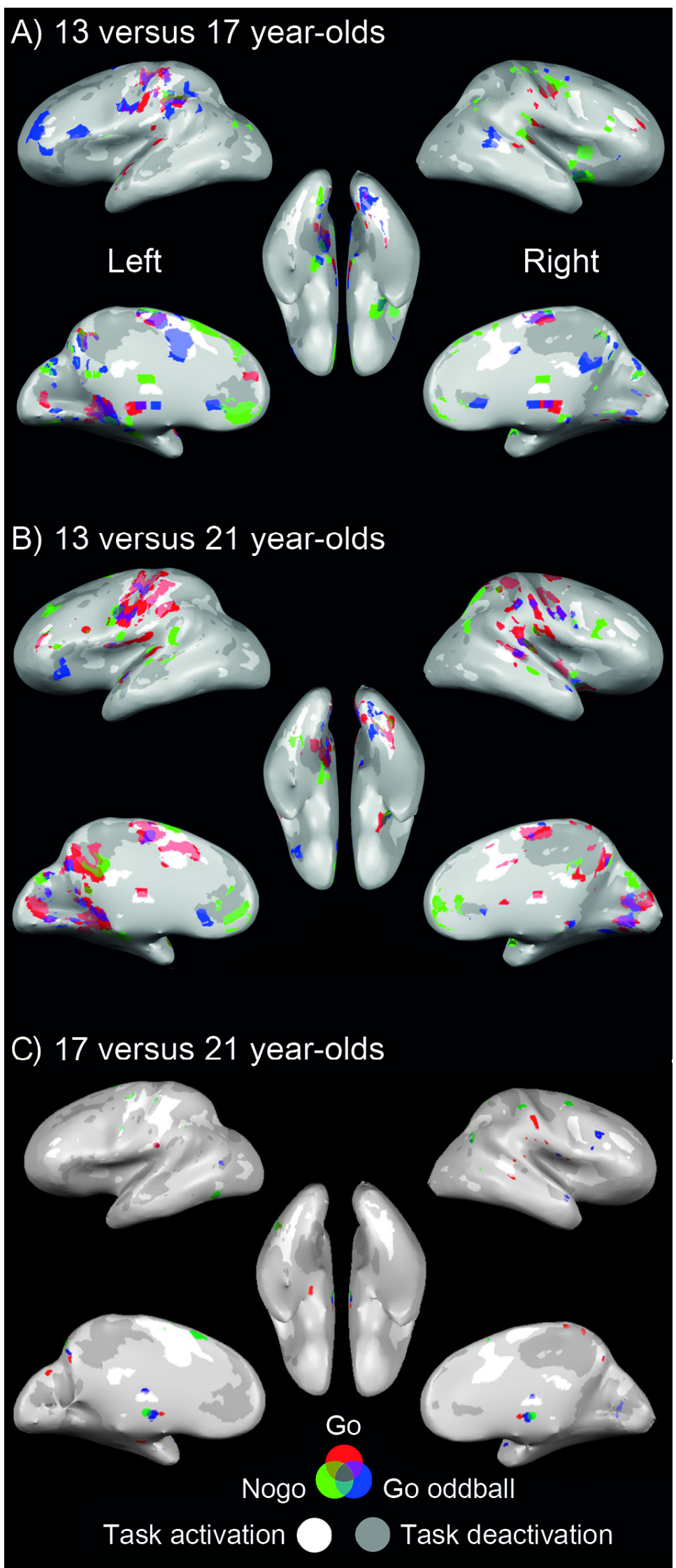




\section{Chapter 5}

Figure 4. Multivariate pattern classification voxel weight maps showing the spatial distribution of brain areas contributing to age group discrimination based on task-induced activation. Of all the voxels retained in the best iteration averaged weight maps for a particular comparison, the $30 \%$ most discriminating voxels (i.e., with the highest weights) are visualized per age-group and task condition comparisons. Weight maps are grouped per age group comparison for 13 versus 17 year-olds (A), 13 versus 21 year-olds (B), and 17 versus 21 year-olds (C), respectively. Within each age group comparison different colors represent the results for different task conditions: Go (red), Go oddball (blue) and Nogo (green) trials. The coloured weight maps are presented superimposed on the functional (Fig. 4 legend continued) activation maps that visualize the task positive (white, thresholded at $\mathrm{t}=2.44$, FDR-pb0.05) and task negative (dark gray, thresholded at $\mathrm{t}=2.23$, FDRpb0.05) responding areas independent of task condition and age group (cf. Fig. 2). This lay-out of task activated and deactivated regions serves as reference frame for interpreting the location of developmental findings. 


\section{A) ROls that overlapped with task negative responding areas}

vmPFC (9 45 9)

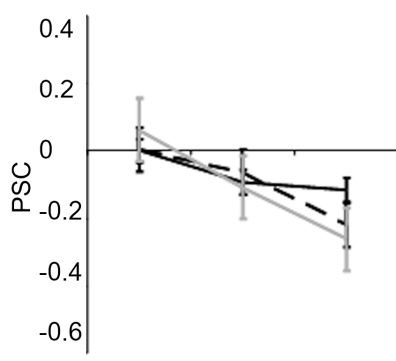

$\operatorname{PCC}(-6-4233)$

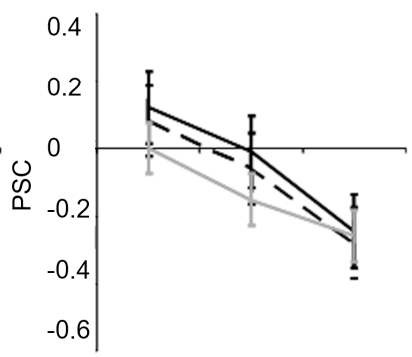

B) ROIs that overlapped with task positive responding areas

SMA $(0-951)$

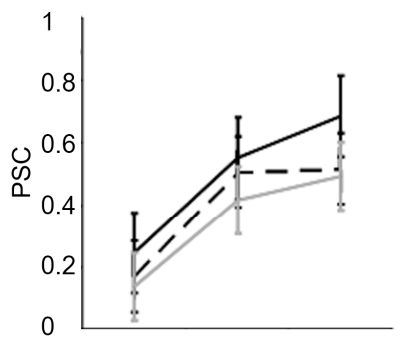

IPS (27 -66 33)

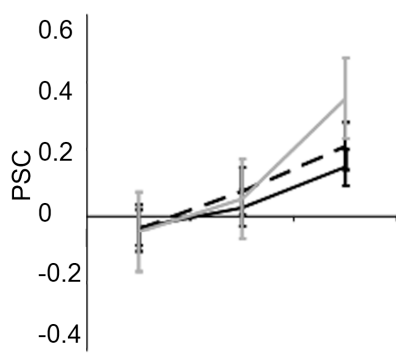

dACC (0 18 42)

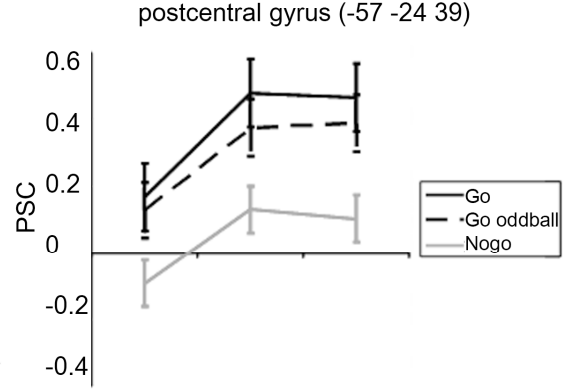

FEF (27 -15 57)

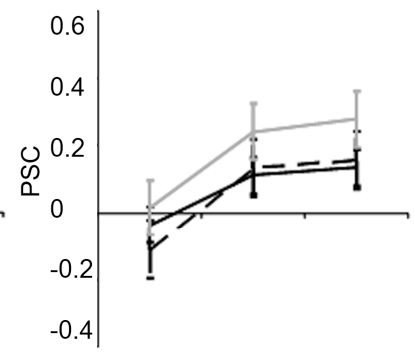

anterior insula (30 18 6)

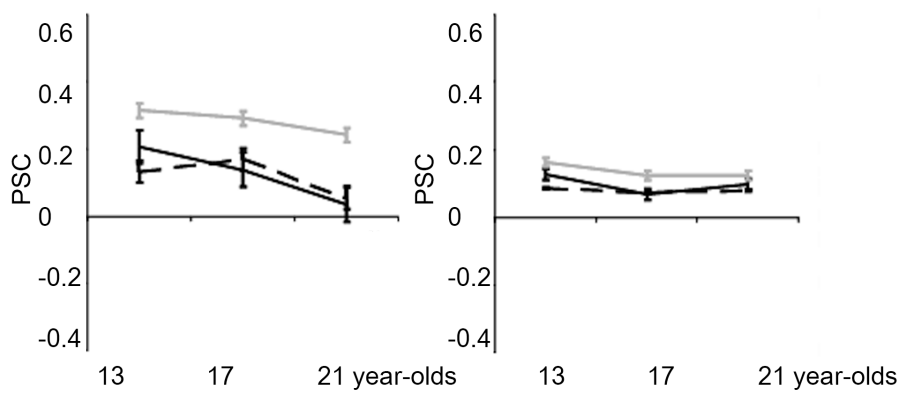

Figure 5. Percent signal changes in ROIs that contributed to age group classification. In each ROI mean percent signal changes are shown with error bars indicating standard error of the mean, separately for each trial type (Go (black), Go oddball (striped), Nogo (gray)). vmPFC = ventral medial prefrontal cortex; PCC = posterior cingulate cortex; SMA = supplementary motor area; IPS = intraparietal sulcus; FEF $=$ frontal eye field; $\mathrm{dACC}=$ dorsal part of anterior cingulate cortex. 


\section{$\underline{\text { Discussion }}$}

Multivariate pattern (MVP) analysis allowed a reliable classification of adolescent age groups of respectively 13, 17 and 21 year-olds based on their activation pattern during a simple gonogo task. The MVP results showed that developmental differences in task activation are spatially distributed throughout the brain, affecting the responsiveness of a wide range of cortical and subcortical structures. We showed that these distributed age-distinctive patterns generalize from one cognitive task to a very different challenging decision making task. This suggests that the maturation in task-induced activation observed here is driven by common cognitive processes that span across cognitive tasks, as opposed to exclusive task-specific processes. When we looked at differences in the wiring of the brain that may underlie these differences in brain function we could confirm the recent suggestion that long range connectivity strength tends to increase within the time window of adolescence. We also found that voxels contributing to age group classification based on task-induced activation were more involved in long range communication than voxels that did not contribute. However, we did not find a strong relationship between task-related and connectivity maturation, as the voxels showing functional maturation did not significantly overlap with voxels showing stronger connectivity maturation. Consequently, if functional rewiring of the brain is the driving force behind the changed functional responsiveness of the brain, it is not so in a simple and direct way.

Widespread age-related differences in task-induced activation

The MVP classification of task-induced brain activation maps allowed establishing well above chance to what age group a particular brain map belonged, with accuracies mostly in the 70-80\% range. For the 17-21 year old comparison, this level of significant classification was reached only by applying initial voxel selection in combination with more fine-grained MVP parameters. This result suggests that a larger portion of the brain contains at least some developmental information between 13 and 17 years, than between 17 and 21 years. However, when the same adjusted parameters were used in the younger age comparison the results were very similar to the older age comparison, both in terms of accuracies and the number of features/voxels yielding the best result. The age-related difference in the number of voxels that can contribute to age classification was mirrored by the stronger improvement in performance efficiency from 13 to 17 years of age than from 17 to 21 years of age and agrees with the estimates in other studies that mature levels of response inhibition are attained around 14-15 years of age (e.g. Luna et al., 2004). Despite the developmental slowing after 17 years, the classification accuracies were as high for the 17-21 year-olds as for the other age group comparisons without initial voxel selection, and their contributing voxels were located as widely spread across the brain.

The spatial distribution of contributing voxels over the whole brain is in line with structural MRI studies of development, that typically find widespread differences in subcortical as well as cortical structures. Cerebral changes mostly involve association cortex in the major lobes, and the white matter tracts between them (Giedd, 2004; Giedd et al., 2009; Giorgio et al., 2010; Gogtay et al., 2004). Also, functional connectivity changes with age mostly take place between spatially remote areas in widespread brain networks, such as anterior cingulate-insular connections in the task related networks and posterior cingulate- ventral medial prefrontal connections in the default mode 
network (Fair et al., 2007a, 2008; Kelly et al., 2009). The voxels that contributed to task-activation based age group classification considerably overlapped with brain areas that were either activated or deactivated during execution of the gonogo task. Some voxels that contributed to age group classification are included in the default mode network (e.g. Greicius et al., 2003), whereas other contributing voxels can be localized to the task-activated functional networks, namely sensory- motor, attention, visual processing, salience and central executive networks (e.g. Damoiseaux et al., 2006). These functional networks have been described during task execution and resting state functional imaging and are consistent between subjects and sessions (Beckmann et al., 2005; Damoiseaux et al., 2006; De Luca et al., 2006; Dosenbach et al., 2007; Fox et al., 2006b; Raichle et al., 2001; Smith et al., 2009). In age-sensitive voxels located in default mode regions, task-induced deactivation increased systematically with age from 13 to 21 years. In age-affected voxels located in task positive regions, the BOLD response increased with age, although the exact developmental trajectory differed somewhat for different regions. In the motor and visual motor related areas investigated (post-central gyrus, supplementary motor area, frontal eye fields) percent signal change values increased between 13 and 21 years. However, in the salience network related anterior cingulate cortex and anterior insula (Sridharan et al., 2008), no significant percent signal change was observed in the post hoc ROI analyses. Although these areas did contribute to age group discrimination, this effect was not strong enough and/or consistent enough throughout the voxels in each ROI to be detected in the post hoc ROI analyses. The spatially distributed age-related increase in functional responsiveness of both task-related and default mode brain networks is in line with previous developmental fMRI studies (e.g. Keulers et al., 2011; Luna et al., 2010; Rubia et al., 2006) as well as with maturational differences in functional connectivity between these areas in this age period (Fair et al., 2007a, 2008; Kelly et al., 2009).

In contrast to the good age discrimination ability of the MVP analysis, the univariate GLM analysis revealed very few, small clusters of voxels with age-related differences in activation, almost all of which occurred in the 13 to 21 year-old comparison. The difference in sensitivity of the two techniques arises because the voxels whose signal change with age is large enough to reach statistical significance on its own are only a small portion of all the voxels whose signal is modulated by age (Poldrack et al., 2009). In line with this, the regions showing maturation according to the GLM analysis all fell within the larger regions highlighted in the MVP analyses. This result shows that a univariate voxel-wise approach lacks the sensitivity to reveal subtle and distributed age differences in brain activation, for which a multivariate approach is better suited. 


\section{Chapter 5}

A) Short range $0-30 \mathrm{~mm}$

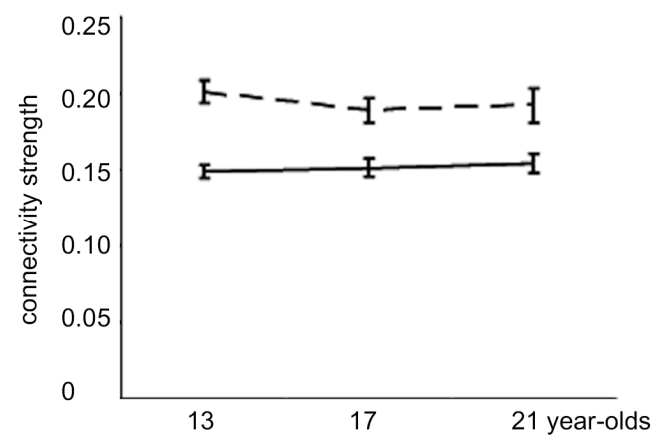

B) Short range $30-60 \mathrm{~mm}$

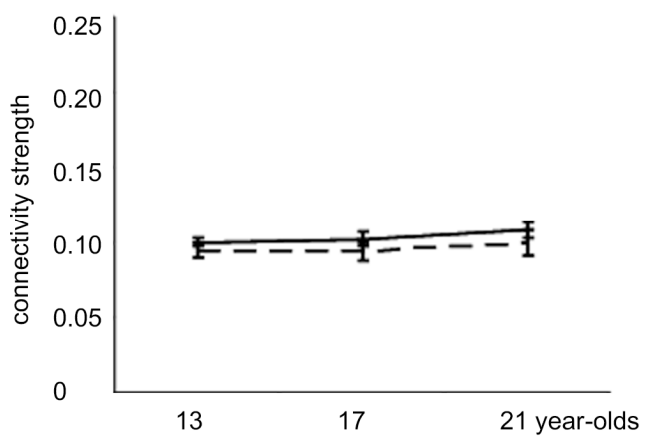

C) Long range $60-90 \mathrm{~mm}$

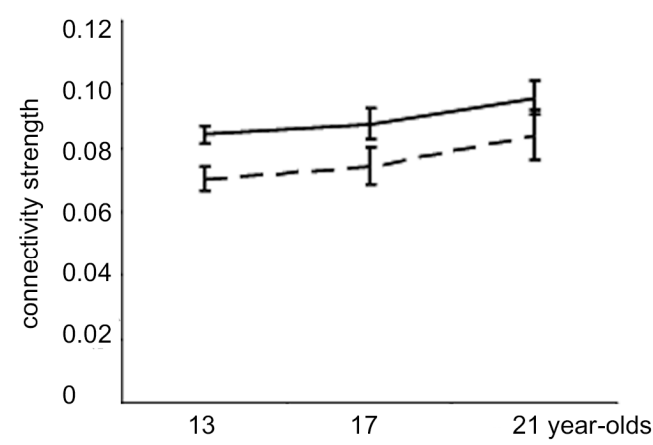

D) Long range $90-120 \mathrm{~mm}$

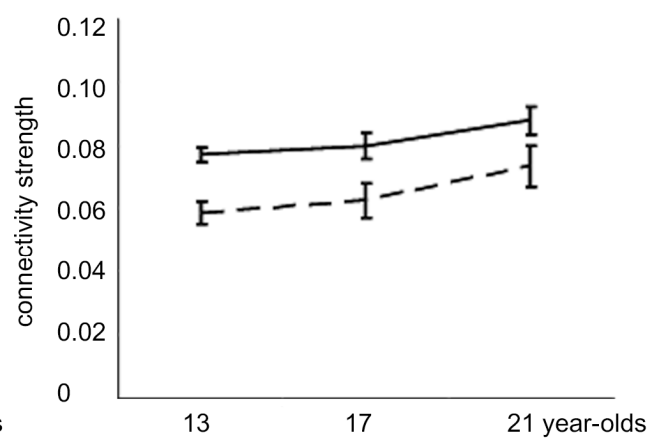

E) Long range $>120 \mathrm{~mm}$

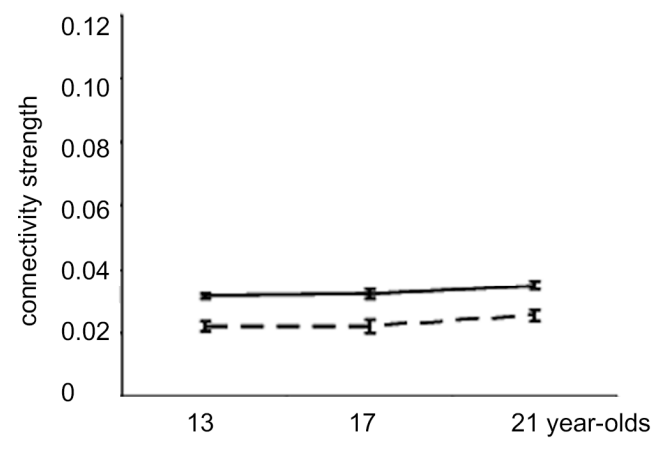

F) Interaction effect mask $x$ distance range

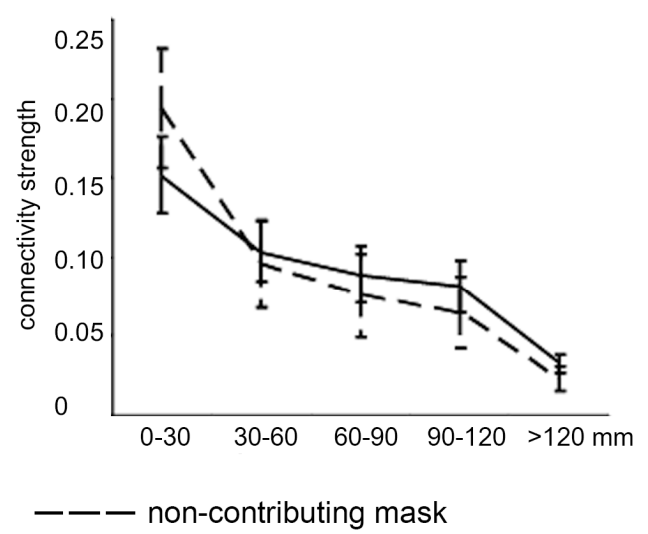

Figure 6. Short and long range functional connectivity strengths for 13, 17 and 21 year-olds adolescents. The mean connectivity strength is shown for each age group, for different short $(A, B)$ and long $(C, D, E)$ range distances. (F) Shows the interaction effect of mask $\times$ distance range. Error bars indicate the standard error of the mean. The black lines represent the contributing mask, consisting of voxels that contributed to age group discrimination as revealed by the MVP analyses based on task-induced (de)activation. The gray lines represent the non-contributing mask consisting of randomly selected voxels that did not contribute to age group classification. 
Generalization of age-distinctive functional patterns

The age-distinctive patterns revealed by the MVP analyses proved to generalize from one cognitive task to a totally different task in the same group of participants. The classifier trained on the gonogo data was able to accurately predict adolescent age groups based on brain activation maps during a very different gambling task, and vice versa. These results suggest that the age group classification is driven by patterns of difference in activation that are common across different cognitive tasks, as opposed to specific properties of a particular task or condition. Even stronger evidence for this generality of functional brain maturation comes from the classification of response inhibition trials in the gambling task (none-gambling trials), by a classifier trained on response execution trials in the gonogo task (Go and Go oddball trials), and vice versa. Thus, brain maturation as revealed by task-induced activity involves a pattern of differences that persists over task paradigms or task-specific cognitive demands. This task-independency is reminiscent of the multiple demand property of some prefrontal areas that are activated during the execution of a wide range of cognitive tasks (e.g. Derrfuss et al., 2004; Duncan, 2010; Duncan and Owen, 2000; Poldrack et al., 2009; Stiers et al., 2010). The dominance of this network in task execution is illustrated by the strong task-related signal change observed in these areas in any task condition, which is much stronger than the subtle modulations that arise within the task-related functional networks in response to the specific task demands (Stiers et al., 2010; Woolgar et al., 2010). The generality of functional maturation patterns across cognitive tasks suggests that the developmental changes that take place during adolescence also affect the brain at this basic level of functioning, as opposed to changes restricted to specific processes. Consequently, the functional repercussions of maturation are visible in the activation patterns of a range of tasks, and in a wide range of brain areas beyond this central network that are also engaged during task execution (e.g., sensory and motor networks). That the age- related patterns of functional maturation also include many voxels than do not converge with the task-related brain areas may seem inconsistent with this interpretation. This apparent inconsistency emerges from the fact that the task-related areas were also identified with a univariate GLM analysis. It is likely that also for taskrelated fMRI the number of significantly activated voxels is just a portion of all the voxels whose signal strength is modulated by task execution. Hence, many more voxels are involved in task execution than are made visible by univariate statistical analysis. Both of the tasks included in the present study are cognitive stimulus-response tasks. The functional operations common to these attentive performance tasks are response control, attention mechanisms, interference handling, error monitoring, memory retrieval, etc. To further test the generality of adolescent functional maturation it would be interesting in future research to include very different tasks, for example, less structured tasks such as listening to music (Sridharan et al., 2008), or self-reflective tasks as have been associated with the default mode network (Buckner et al., 2008), which is complementary to the task-performance network activated by the Gonogo and Gambling task. The generality observed in the current study does not exclude the possibility that functional maturation may also be evident in specific neurocognitive functions. The generality tested here merely investigates whether a particular brain-wide developmental pattern found in one task is also present in another task, and does not exclude the possibility of additional patterns confined to brain regions associated with particular neurocognitive functions. Cohen et al. (2010), for instance, using multivariate pattern analysis on data from a stop- task, could only predict age from a response 


\section{Chapter 5}

inhibition specific activation map and not from the basic activation map derived from the go-trials. This selectivity could indicate that particularly the neural mechanisms underlying the ability to abort an already ongoing response are susceptible to maturation changes. However, their different results could also reflect differences in methodological choices (age range investigated (9-30 years), heterogeneity of age groups, participant exclusion procedures, etc.). Future studies will have to include a wider range of tasks, both in terms of the neurocognitive function addressed and the task paradigms to invoke them, to address the question of how general functional maturation really is, in terms of central executive of specific cognitive demands, operation modes of the brain, the age range for which it holds, etc.

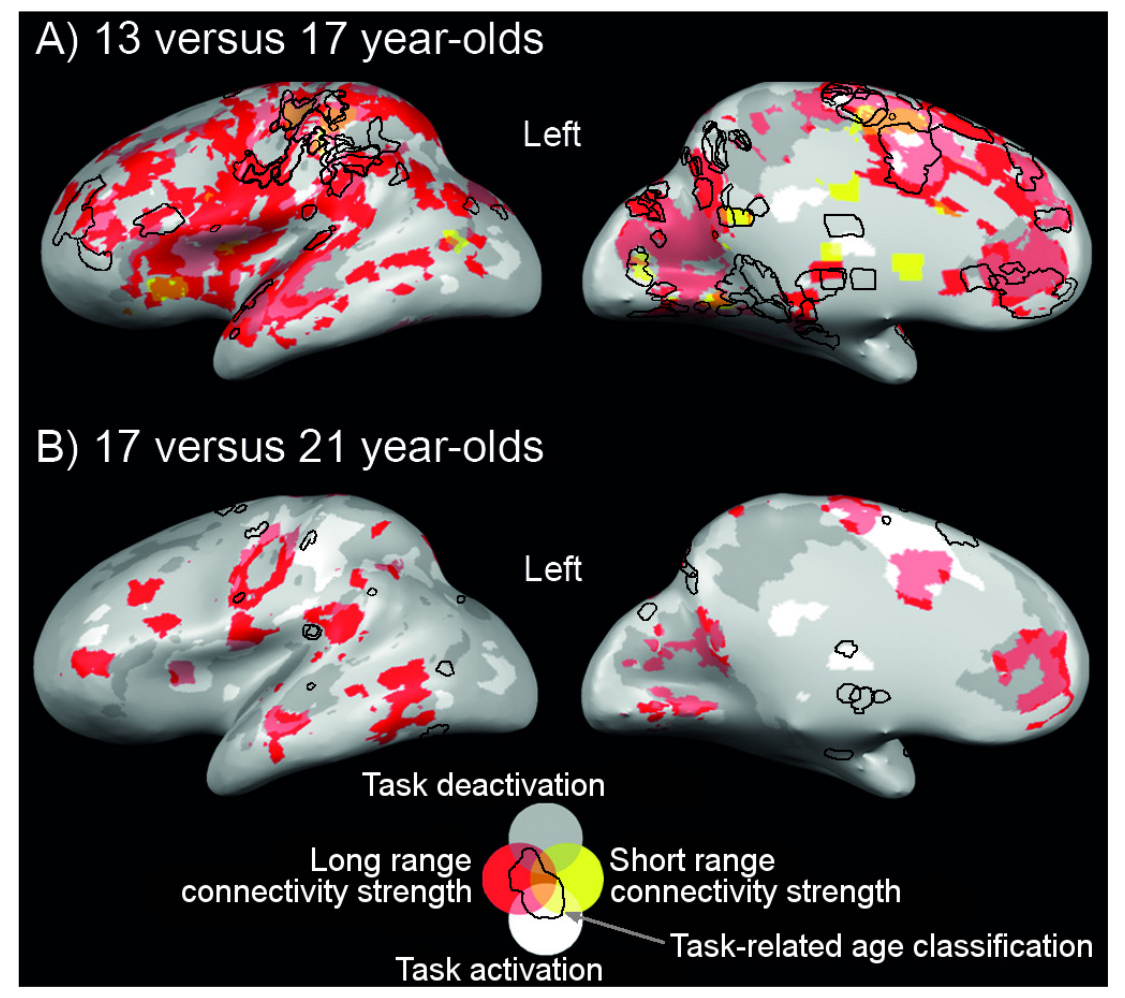

Figure 7. Pattern of brain areas contributing to age group discrimination revealed by multivariate pattern classification analyses based on functional connectivity. Spatial pat- tern of voxels contributing to age group discrimination revealed by multivariate pattern classification analyses based on functional connectivity strength, separately for short $(0-60 \mathrm{~mm}$; presented in yellow) and long $(>60 \mathrm{~mm}$; presented in red) range distances. The $30 \%$ most discriminating voxels of the weight maps corresponding to the best iteration are visualized here. Weight maps are presented for, respectively, 13 versus 17 year-olds (A) and 17 versus 21 year-olds (B).

The relation between maturation of task-induced activation and functional connectivity

Recent resting state imaging studies revealed maturational differences in functional connectivity within several brain networks defined by ROIs. These comprised increased long range and/or decreased short range connectivity strength between childhood and adulthood, with an intermediate, however often not significant, position for adolescents (Fair et al., 2007a, 2009; Kelly et al., 2009). We extended these previous findings on functional connectivity maturation by showing similar patterns within the time window 
of adolescence, using confined, homogeneous age groups and a whole brain approach based on the low- frequency background fluctuations in task-related time series data. Our univariate analyses, which averaged connectivity measures over voxels, suggests that the shift from local to long range connectivity in this adolescent age window is subtle and not always statistically significant. In contrast, MVP classification analysis on whole-brain long range connectivity strength values obtained high accuracies in differentiating between the adolescent age groups of respectively 13, 17 and 21 years. This once more confirms the higher sensitivity of multivariate over univariate methods for developmental data. Maturational changes in short range connectivity tend to occur only before age 17 and seem to confine to areas with a preference for local connectivity, such as SMA, visual and somatosensory regions (Sepulcre et al., 2010). The whole brain approach of the present study in examining functional connectivity, as opposed to the ROI based approach, with ROIs derived from adult imaging studies (Fair et al., 2007a, 2008, 2009; Kelly et al., 2009), shows how wide spread throughout the brain the maturation of long range functional connections is. The advantage of the whole brain approach is that it is data- driven and prevents a priori exclusion of relevant information, for example regions that are included in functional networks in adolescents but not in adults. The trend of increased long range connectivity strength with age was, however, the same for voxels that did and did not contribute to age group classification based on task-induced activation. Correspondingly, the MVP results based on whole brain functional connectivity strength showed that age-related differences affect a large number of voxels distributed throughout the brain, and are not at all confined to regions that showed task activation maturation. In fact, the extent of overlap between voxels contributing to age group classification based on task-induced activation on the one hand and long range functional connectivity on the other hand was small relative to the total amount of voxels contributing to long range connectivity classification. This suggests that at least at the whole brain level the altered responsiveness of the brain observed during the execution of cognitive tasks does not directly go back to a difference in functional connectivity: many voxels that do mature in their task-response do not play an important role in connectivity-based age discrimination, while even more voxels that do not substantially contribute to task-response based age discrimination do show strong maturation in functional connectivity. It should be noted that task execution invokes subtle differences in the low frequency functional connectivity, compared to a state of rest, during execution itself (Fair et al., 2007b; Fox et al., 2006b) as well as in periods of rest following it (Dale et al., 2010; Hasson et al., 2009). While these differences may have affected the observed relationship between task-activity and functional connectivity maturation in our study, the effect can be expected to be small given that the reported task-related changes in functional connectivity are relatively small. A last finding was that voxels that contribute to task-related age group classification have more connections over long ranges and less short range connections compared with non contributing voxels. This only means, however, that voxels that show an age-related difference in their responsiveness during cognitive task execution are particularly involved in long range communication, i.e., overlap with the large-scale brain networks. A difficult question to answer, and a challenge for future research, is how developmental changes in task-induced functional responsiveness of the brain are related to changes in brain anatomy, and particularly in large scale functional interactions between areas. The present results make clear that this relationship is not simply that voxels that mature in whole brain functional connectivity are also the voxels that will differ in their responsiveness. Rather, our results suggest that connectivity 


\section{Chapter 5}

maturation during adolescence takes place at the brain wide scale, affecting sensory as well as association areas. Because long-range functional connectivity only reflects communication between areas, this result does not necessary disagree with anatomical imaging studies that localize adolescent brain maturation more to association cortex (e.g. Gogtay et al., 2004; Huttenlocher and Dabholkar, 1997). The functional connectivity results merely show that communication in the brain has become different at a large, brain-wide scale. The net effect is improved behavioural performance as well as changed brain-induced activation in almost all levels of cognitive functioning from simple response control to complex decision making.

\section{$\underline{\text { References }}$}

Achenbach TM, Rescorla LA (2001) Manual for the ASEBA School-age Forms \& Profiles. University of Vermont, Research Centre for Children, Youth and Families, Burlington, VT.

Ardila A, Rosselli M, Matute E, Guajardo S (2005) The influence of the parents' educational level on the development of executive functions. Dev Neuropsychol 28:539-560.

Beckmann CF, De Luca M, Devlin JT, Smith SM (2005) Investigations into resting state connectivity using independent component analysis. Philos Trans R Soc B 360:1001-1013.

Berl MM, Vaidya CJ, Gaillard WD (2006) Functional imaging of developmental and adaptive changes in neurocognition. NeuroImage 30:679-691.

Brown TT, Lugar HM, Coalson RS, Miezin FM, Petersen SE, Schlaggar BL (2005) Developmental changes in human cerebral functional organization for word generation. Cereb Cortex 15:275-290.

Buckner RL, Andrews-Hanna JR, Schacter DL (2008) The brain's default network: anatomy, function, and relevance to disease. Ann N Y Acad Sci 1124:1-38.

Bullmore E, Sporns O (2009) Complex brain networks: graph theoretical analysis of structural and functional systems. Nat Rev Neurosci 10:186-198.

Calhoun VD, Kiehl KA, Pearlson GD (2008) Modulation of temporally coherent brain networks estimated using ICA at rest and during cognitive tasks. Hum Brain Mapp 29:828-838.

Casey BJ, Getz S, Galvan A (2008) The adolescent brain. Dev Rev 28:62-77.

Chikazoe J, Jimura K, Asari T, Yamashita K, Morimoto H, Hirose S, et al. (2009) Functional dissociation in right inferior frontal cortex during performance of go/ no-go task. Cereb Cortex 19:146-152.

Cohen JR, Asarnow RF, Sabb FW, Bilder RM, Bookheimer SY, Knowlton BJ et al. (2010) Decoding developmental differences and individual variability in response inhibition through predictive analyses across individuals. Front Hum Neurosci 4:1-12.

Cole MW, Schneider W (2007) The cognitive control network: integrated cortical regions with dissociable functions. NeuroImage 37:343-360.

Cole MW, Pathak S, Schneider W (2010) Identifying the brain's most globally connected regions. NeuroImage 49:3132-3148.

Corbetta M, Shulman GL (2002) Control of goal-directed and stimulus-driven attention in the brain. Nat Rev Neurosci 3:201-215.

Cox D, Savoy R (2003) Functional magnetic resonance (fMRI) "brain reading": detecting and classifying distributed patterns of fMRI activity in human visual cortex. NeuroImage 19:261-270. 
Crone EA, Wendelkern C, Donohue S, van Leijenhorst L, Bunge SA (2006) Neurocognitive development of the ability to manipulate information in working memory. Proc Natl Acad Sci U S A 103:9315-9320.

Dahl RE (2004) Adolescent brain development: a period of vulnerabilities and opportunities. Keynote address. Ann N Y Acad Sci 1021:1-22.

Dale SW, Buckner RL, Schacter DL (2010) Correlated low-frequency BOLD fluctua- tions in the resting human brain are modulated by recent experience in category-preferential visual regions. Cereb Cortex 20:1997-2006.

Damoiseaux JS, Rombouts SARB, Barkhof F, Scheltens P, Stam CJ, Smith, SM, et al. (2006) Consistent resting-state networks across healthy subjects. Proc Natl Acad Sci U S A 103:13848-13853.

De Luca M, Beckmann CF, De Stefano N, Matthews PM, Smith SM (2006) fMRI resting state networks define distinct modes of long-distance interactions in the human brain. NeuroImage 29:1359-1367.

De Martino F, Valente G, Staeren N, Ashburner J, Goebel R, Formisano E (2008) Combining multivariate voxel selection and support vector machines for mapping and classification of fMRI spatial patterns. NeuroImage 43:44-58.

Derrfuss J, Brass M, von Cramon DY (2004) Cognitive control in the posterior frontolateral cortex: evidence from common activations in task coordination, interference control, and working memory. NeuroImage 23:604-612.

Directoraat-Generaal voor de Arbeidsvoorziening, 1989. Handleiding voor de functiea- nalyse [Function analyses manual]. SDU uitgeverij, Den Haag.

Dosenbach NUF, Visscher KM, Palmer ED, Miezin FM, Wenger KK, Kang HC, et al. (2006) A core system for the implementation of task sets. Neuron 50:799-812.

Dosenbach NU, Fair DA, Miezin FM, Cohen AL, Wenger KK, Dosenbach RAT, et al. (2007) Distinct brain networks for adaptive and stable task control in humans. Proc Natl Acad Sci U S A 104:11073-11078.

Dosenbach NUF, Nardos B, Cohen AL, Fair DA, Power JD, Church JA, et al. (2010) Prediction of individual brain maturity using fMRI. Science 329:1358-1361.

Duncan, J (2010) The multiple-demand (MD) system of the primate brain: mental programs for intelligent behaviour. Trends Cogn Sci 14:172-179.

Duncan J, Owen AM (2000) Common regions of the human frontal lobe recruited by diverse cognitive demands. Trends Neurosci 23:475-483.

Dunn LM, Dunn LM (2005) Peabody Picture Vocabulary Test-III-NL. Handleiding [Manual]. Harcourt Assessment B.V, Amsterdam.

Durston S, Casey BJ (2006) What have we learned about cognitive development from neuroimaging? Neuropsychologia 44:2149-2157.

Durston S, Davidson MC Tottenham N Galvan A, Spicer J, Fossella JA et al. (2006) A shift from diffuse to focal cortical activity with development. Dev Sci 9:1-20.

Fair DA, Dosenbach NUF, Church JA, Cohen AL, Brahmbhatt S, Miezin FM, et al. (2007a) Development of distinct control networks through segregation and integration. Proc Natl Acad Sci U S A 104:13507-13512.

Fair DA, Schlaggar BL, Cohen AL, Miezin FM, Dosenbach NUF, Wenger KK, et al. (2007b) A method for using blocked and event-related fMRI data to study "resting state" functional connectivity. NeuroImage 35:396-405.

Fair DA, Cohen AL, Dosenbach NUF, Church JA, Miezin FM, Barch DM, et al. (2008) The maturing architecture of the brain's default network. Proc Natl Acad Sci U S A 105:4028-4032.

Fair DA, Cohen AL, Power JD, Dosenbach NUF, Church JA, Miezin FM, et al. (2009) Functional brain networks develop from a "Local to Distributed" organiza- tion. PLoS Comput Biol 5:1-14. 


\section{Chapter 5}

Fan Y, Rao H, Hurt H, Giannetta J, Korczykowski M, Shera D, et al. (2007) Multivariate examination of brain abnormality using both structural and functional MRI. NeuroImage 36:1189-1199.

Formisano E, De Martino F, Bonte M, Goebel R (2008) "Who" is saying “what"? Brain-based decoding of human voice and speech. Science 322:970-973.

Fox MD Corbetta M, Snyder AZ, Vincent JL, Raichle ME (2006a) Spontaneous neuronal activity distinguishes human dorsal and ventral attention systems. Proc Natl Acad Sci U S A 103:10046-10051.

Fox MD, Snyder AZ, Zacks JM, Raichle ME (2006b) Coherent spontaneous activity accounts for trial-to-trial variability in human evoked brain responses. Nat Neurosci 9:23-25.

Friston KJ, Williams S, Howard R, Frackowiak RSJ, Turner R (1996) Movement- related effects in fMRI time-series. Magn Reson Med 35:346-355.

Geier CF, Garver K, Terwilliger R, Luna B (2009) Development of working memory maintenance. J. Neurophysiol. 101:84-99.

Genovese CR, Lazar NA, Nicols T (2002) Thresholding of statistical maps in functional neuroimaging using the false discovery rate. NeuroImage 15:870-878.

Giedd JN (2004) Structural magnetic resonance imaging of the adolescent brain. Ann N Y Acad Sci 1021:7785 .

Giedd JN, Lalonde FM, Celano MJ, White SL, Wallace GL, Lee NR, et al. (2009) Anatomical brain magnetic resonance imaging of typically developing children and adolescents. J Am Acad Child Adolesc Psychiatry 48:465-470.

Giorgio A, Watkins KE, Chadwick M, James S, Winmill L, Douaud G, et al. (2010) Longitudinal changes in grey and white matter during adolescence. NeuroImage 49: 94-103.

Glantz LA, Gilmore JH, Hamer RM, Lieberman JA, Jarskog LF (2007) Synaptophysin and postsynaptic density protein 95 in the human prefrontal cortex from mid-gestation into early adulthood. Neuroscience 149:582-591.

Gogtay N, Giedd JN, Lusk L, Hayashi KM, Greenstein D, Vaituzis AC et al. (2004) Dynamic mapping of human cortical development during childhood through early adulthood. Proc Natl Acad Sci U S A 101:81748179 .

Greicius MD, Krasnow B, Reiss AL, Menon V (2003) Functional connectivity in the resting brain: a network analysis of the default mode hypothesis. Proc Natl Acad Sci U S A 100:253-258.

Guyon I, Weston J, Barnhill S, Vapnik V (2002) Gene selection for cancer classification using support vector machines. Mach Learn 46:389-422.

Hampshire A, Chamberlain SR, Monti MM, Duncan J, Owen AM (2010) The role of the right inferior frontal gyrus: inhibition and attentional control. NeuroImage 50:1313-1319.

Hasson U, Nusbaum HC, Small SL (2009) Task-dependent organization of brain regions active during rest. Proc Natl Acad Sci U S A 106:10841-10846.

Haxby JV, Gobbini MI, Furey ML, Ishai A, Aschouten JL, Pietrini P (2001) Distributed and overlapping representations of faces and objects in ventral temporal cortex. Science 293:2425-2430.

Haynes JD Rees G (2006) Decoding mental states from brain activity in humans. Nat Rev Neurosci 7:523534.

Huttenlocher PR, Dabholkar AS (1997) Regional differences in synaptogenesis in human cerebral cortex. J Comp Neurol 387:167-178. 
Johnstone T, Ores Walsh KS, Greischar LL, Alexander AL, Fox AS, Davidson R, et al. (2006) Motion correction and the use of motion covariates in multiple-subject fMRI analysis. Hum Brain Mapp 27:779-788.

Kelly AMC, Di Martino A, Uddin LQ, Shehzad Z, Gee1 DG, Reiss PT, et al. (2009) Development of anterior cingulate functional connectivity from late childhood to early adulthood. Cereb Cortex 19:640-657.

Keulers EHH, Stiers P, Jolles J (2011) Developmental changes between ages 13 and 21 years in the extent and magnitude of the BOLD response during decision making. NeuroImage 54:1442-1454.

Klenberg L, Korkman M, Lahti-Nuuttila P (2001) Differential development of attention and executive functions in 3- to 12-year-old Finnish children. Dev Neuropsychol 20:407-428.

Lebel C, Walker L, Leemans A, Phillips L, Beaulieu C (2008) Microstructural maturation of the human brain from childhood to adulthood. NeuroImage 40:1044-1055.

Luna B, Garver KE, Urban TA, Lazar NA, Sweeney JA (2004) Maturation of cognitive processes from late childhood to adulthood. Child Dev 75:1357-1372.

Luna B, Velanova K, Geier CF (2010) Methodological approaches in developmental neuroimaging studies. Hum Brain Mapp 31:863-871.

Mayer AR, Franco AR, Ling J, Canive JM (2007) Assessment and quantification of head motion in neuropsychiatric functional imaging research as applied to schizophrenia. J Int Neuropsychol Soc 13:839845 .

Mennes M, Kelly C, Zuo XN, Di Martino A, Biswal B, Castellanos XF, et al. (2010) Inter-individual differences in resting state functional connectivity predict task- induced BOLD activity. NeuroImage 50:1690-1701.

Mourao-Miranda J, Bokde ALW, Born C, Hampel H, Stetter M (2005) Classifying brain states and determining the discriminating activation patterns: Support Vector Machine on functional MRI data. NeuroImage 28:980-995.

Pereira F, Mitchell T, Botvinick M (2009) Machine learning classifiers and fMRI: a tutorial overview. NeuroImage 45:S199-S209.

Poldrack RA, Halchenko Y, Hanson SJ (2009) Decoding the large-scale structure of brain function by classifying mental states across individuals. Psychol Sci 20:1364-1372.

Power JD, Fair DA, Schlaggar BL, Petersen SE (2010) The development of human functional brain networks. Neuron 67:735-748.

Raichle ME, MacLeod AM, Snyder AZ, Powers WJ, Gusnard DA, Shulman GL (2001) A default mode of brain function. Proc Natl Acad Sci 98:676-682.

Raven J, Raven JC, Court JH (1998) Manual for Raven's Progressive Matrices and Vocabulary Scales. Section 1: General Overview. Harcourt Assessment, San Antonio TX.

Rubia K, Smith AB, Woolley J, Nosarti C, Heyman I, Taylor E, et al. (2006) Progressive increase of frontostriatal brain activation from childhood to adulthood during event-related tasks of cognitive control. Hum Brain Mapp 27:973-993.

Rubia K, Smith AB, Taylor E, Brammer M (2007) Linear age-correlated functional development of right inferior fronto-striato-cerebellar networks during response inhibition and anterior cingulate during errorrelated processes. Hum Brain Mapp 28:1163-1177.

Ryali S, Supekar K, Abrams DA, Menon V (2010) Sparse logistic regression for whole brain classification of fMRI data. NeuroImage 51:752-764.

Sepulcre J, Liu H, Talukdar T, Martincorena I, Yeo BT, Buckner RL (2010) The organization of local and distant functional connectivity in the human brain. PLoS Comp Biol 10:e1000808. 


\section{Chapter 5}

Slifer KJ, Cataldo MF, Cataldo MD, Llorente AM, Gerson AC (1993) Behavior analysis of motion control for pediatric neuroimaging. J Appl Behav Anal 269:469-470.

Smith SM, Fox PT, Miller KL, Glahn, DC, Fox PM, Mackay CE, et al. (2009) Correspondence of the brain's functional architecture during activation and rest. Proc Natl Acad Sci U S A 106:13040-13045.

Sowell ER, Thompson PM, Leonard CM, Welcome SE, Kan E, Toga AW (2004) Longitudinal mapping of cortical thickness and brain growth in normal children. J Neurosci 24:8223-8231.

Sridharan D, Levitin DJ, Menon V (2008) A critical role for the right fronto-insular cortex in switching between central-executive and default-mode networks. Proc Natl Acad Sci U S A 105:12569-12574.

Staeren N, Renvall H, De Martino F, Goebel R, Formisano E (2009) Sound categories are represented as distributed patterns in the human auditory cortex. Curr Biol 19:498-502.

Steinberg L (2008) A social neuroscience perspective on adolescent risk-taking. Dev Rev 28:78-106.

Steinberg L, Albert D, Cauffman E, Banich M, Graham S, Woolard J (2008) Age differences in sensation seeking and impulsivity as indexed by behaviour and self report: evidence for a dual systems model. Dev Psychol 44:1764-1778.

Stiers P, Mennes M, Sunaert S (2010) Distributed task coding throughout the multiple demand network of the human frontal-insular cortex. NeuroImage 52:252-262.

Stuss DT, Murphy KJ, Binns MA, Alexander MP (2003) Staying on the job: the frontal lobes control individual performance variability. Brain 126:2363-2380.

Sun D, van Erp TGM, Thompson PM, Bearden CE, Daley M, Kushan L, et al. (2009) Elucidating a magnetic resonance imaging-based neuroanatomic biomarker for psychosis: classification analysis using probabilistic brain atlas and machine learning algorithms. Biol Psychiatry 66:1055-1060.

Suykens JAK, Van Gestel T, De Barbanter J, De Moor B, Vanderwalle J (2002) Least Squares Support Vector Machines. World Scientific Publishing.

Talairach J, Tournoux P (1988) Co-planar Stereotaxic Atlas of the Human Brain. Thieme Medical Publishers Inc, New York.

Tamm L, Menon V, Reiss AL (2002) Maturation of brain function associated with response inhibition. J Am Acad Child Adolesc Psychiatry 41:1231-1238.

van Leijenhorst L, Zanolie K, Van Meel CS, Westenberg PM, Rombouts SARB, Crone EA (2010) What motivates the adolescent? Brain regions mediating reward sensitivity across adolescence. Cereb Cortex 20:6169.

Velanova K, Wheeler ME, Luna B (2009) The maturation of task set-related activation supports late developmental improvements in inhibitory control. J Neurosci 29:12558-12567.

Vogel AC, Power JD, Petersen SE, Schlaggar BL (2010) Development of the brain's functional network architecture. Neuropsychol Rev 20:362-375.

Woolgar A, Thompson R, Bor D, Duncan J (2010) Multi-voxel coding of stimuli, rules, and responses in human frontoparietal cortex. NeuroImage 56:744-752.

Yakovlev PI, Lecours A (1967) The myelogenetic cycles of regional maturation in the brain. In: Minkowski, A. (Ed.), Regional Development of the Brain in Early Life. Blackwell, pp. 3-65.

Yoo S, Choi B, Juh R, Pae C, Lee C (2005) Headmotion analysis during cognitive fMRI examination: application in patients with schizophrenia. Neurosci Res 53:84-90. 


\section{Supplementary material}

Supp Table 1. Overview of task positive and negative responding areas during a pooled gonogo task contrast independent of age group.

\begin{tabular}{|c|c|c|c|c|c|c|}
\hline Brain area & & $\begin{array}{l}\text { TAL } \\
x \quad y \quad z \\
\end{array}$ & Size & T value & $\begin{array}{l}\text { Dosenbach } \\
\text { et al., } 2007\end{array}$ & $\begin{array}{l}\text { Sridharan } \\
\text { et al., } 2008\end{array}$ \\
\hline \multicolumn{7}{|c|}{ POSITIVE RESPONDING AREAS } \\
\hline $\begin{array}{l}\text { supplementary motor area } \\
\text { anterior cingulate cortex }\end{array}$ & \begin{tabular}{|l|}
$\mathrm{L}$ \\
$\mathrm{R}$ \\
\end{tabular} & $\begin{array}{l}-9-648 \\
61233 \\
\end{array}$ & 320 & $\begin{array}{l}11.80 \\
7.49 \\
\end{array}$ & -11046 & 43030 \\
\hline anterior insula & $\mathrm{R}$ & $\begin{array}{l}30129 \\
42123\end{array}$ & 60 & $\begin{array}{l}6.84 \\
6.62\end{array}$ & 36164 & $3725-4$ \\
\hline anterior insula & $\mathrm{L}$ & -33915 & 28 & 6.54 & -35144 & $-3424-6$ \\
\hline posterior insula & $\bar{L}$ & $\begin{array}{lll}-48 & -6 & 12 \\
-42 & -9 & 18\end{array}$ & 29 & $\begin{array}{l}8.18 \\
5.20\end{array}$ & & \\
\hline inferior frontal sulcus & $\mathrm{R}$ & 332730 & 18 & 5.54 & 432234 & 451645 \\
\hline $\begin{array}{l}\text { thalamus } \\
\text { putamen }\end{array}$ & $\overline{\mathrm{L}}$ & $\begin{array}{l}-15-189 \\
-2433 \\
-18315\end{array}$ & 107 & $\begin{array}{l}9.23 \\
7.56 \\
6.17\end{array}$ & $-12-157$ & \\
\hline $\begin{array}{l}\text { thamalus } \\
\text { putamen }\end{array}$ & $\mathrm{R}$ & $\begin{array}{l}9-312 \\
1833\end{array}$ & 32 & $\begin{array}{l}6.26 \\
6.20\end{array}$ & $10-158$ & \\
\hline postcentral gyrus & $\bar{L}$ & $\begin{array}{llll}-42 & -21 & 54 \\
-54 & -21 & 45 \\
-51 & -36 & 48\end{array}$ & 207 & $\begin{array}{l}10.19 \\
8.89 \\
5.73\end{array}$ & & \\
\hline fusiform gyrus & L & $\begin{array}{lll}-39 & -51 & -21 \\
-39 & -60 & -15 \\
-27 & -78 & -9\end{array}$ & 138 & $\begin{array}{l}11.14 \\
9.20 \\
6.65\end{array}$ & $-32-66-29$ & \\
\hline fusiform gyrus & $\mathrm{R}$ & $\begin{array}{l}27-45-21 \\
33-63-9 \\
18-42-18\end{array}$ & 381 & $\begin{array}{l}10.65 \\
10.28 \\
9.92\end{array}$ & $31-61-29$ & \\
\hline lingual gyrus & $\overline{\mathrm{R}}$ & $21-843$ & 24 & 11.50 & $8-824$ & \\
\hline middle occipital gyrus & $\mathrm{L}$ & $-30-813$ & 11 & 7.27 & & \\
\hline \multicolumn{7}{|c|}{ NEGATIVE RESPONDING AREAS } \\
\hline superior frontal sulcus & $\mathrm{L}$ & -24948 & 5 & 5.26 & & \\
\hline inferior frontal sulcus & $\mathrm{L}$ & -512715 & 10 & 5.42 & & \\
\hline posterior insula & $\mathrm{R}$ & $\begin{array}{lll}33 & -18 & 21 \\
42 & -18 & 9 \\
33 & -15 & 12\end{array}$ & 318 & $\begin{array}{l}10.50 \\
10.05 \\
7.79\end{array}$ & & \\
\hline $\begin{array}{l}\text { superior parietal lob } \\
\text { postcentral sulcus } \\
\text { precentral sulcus }\end{array}$ & & $\begin{array}{lll}21 & -39 & 60 \\
21 & -24 & 57 \\
33 & -21 & 39 \\
\end{array}$ & 913 & $\begin{array}{l}10.61 \\
8.60 \\
8.53 \\
\end{array}$ & & \\
\hline $\begin{array}{l}\text { posterior cingulate cortex/ } \\
\text { anterior calcarine sulcus }\end{array}$ & $\mathrm{L}$ & $\begin{array}{llll}-24 & -57 & 15 \\
-12 & -84 & 21 \\
-18 & -72 & 24\end{array}$ & 234 & $\begin{array}{l}6.79 \\
6.52 \\
6.38\end{array}$ & $-11-5713$ & $7-4333$ \\
\hline lingual gyrus & $\mathrm{R}$ & $\begin{array}{l}3-663 \\
12-6618 \\
3-6012\end{array}$ & 236 & $\begin{array}{l}6.87 \\
6.73 \\
3.60\end{array}$ & $8-824$ & \\
\hline parahippocampal gyrus & $\mathrm{L}$ & $\begin{array}{lll}-30 & -36 & -9 \\
-21 & -39 & -9 \\
-27 & -48 & 6\end{array}$ & 101 & $\begin{array}{l}7.17 \\
6.98 \\
5.11\end{array}$ & $-25-44-12$ & \\
\hline parahippocampal gyrus & $\mathrm{R}$ & $21-36-6$ & 7 & 5.73 & $25-44-12$ & \\
\hline parahippocampal gyrus & $\mathrm{R}$ & $24-30-12$ & 5 & 5.26 & & \\
\hline superior temporal gyrus & $\mathrm{L}$ & $-48-246$ & 79 & 8.22 & & \\
\hline
\end{tabular}

Note. The whole brain GLM results described here are significant at the family wise error corrected level $(\mathrm{F}<.05 ; \mathrm{F}=24.64$; voxels $\geq 5)$. In the last two columns the present results are compared with two (Supp Table 1 legend continued) studies that described areas within task positive networks as well as the default mode network (Dosenbach et al. 2007; Sridharan et al. 2008). Size in number of voxels. TAL= Talairach coordinates; $\mathrm{R}=$ right hemisphere; $\mathrm{L}=$ left hemisphere; $\mathrm{VMP}=$ ventral medial nucleus of the thalamus. 
164 
Chapter 6

\section{Discussion}




\section{Synopsis of the thesis research goals and findings}

The present thesis investigated the architecture and connectivity of the macaque and human brain. Tools from network science were employed alongside with in vivo neuroimaging techniques and data from a neuroinformatics database.

Chapter 2 aimed at delineating in vivo the cortical fields of the human lateral frontal cortex (LFC) and unravelling their functional connectivity. We uncovered neuroanatomically realistic "functional modules" that correspond to various putative anatomical substrates/cortical fields revealed in microsctuctural based parcellation studies. The functional circuitry of each putative cortical field can indicate its contribution to LFC function. Additionally, the functional connectivity of the cortical fields seems largely consistent with the anatomical connections of assumed homologues in the macaque LFC. Our approach revealed an organization schema of the cortical fields of the LFC partially supporting dominant models of the human LFC that postulate an organization along the dorsal-ventral and anterior-posterior axis. Such models largely depend on findings from macaque research. Hence, our results offer a parcellation scheme that can function as a platform for future task based/cognitive studies, guide LFC models and link human and macaque research.

While the study described in the previous chapter was not tailored for testing a specific (pre)frontal model, in Chapter 3 we explicitly examined the hierarchy principles and predictions of a model advocating a hierarchical anterior-posterior gradient in the prefrontal cortex. Our formal approach, by employing network analysis tools previously used in systems biology, revealed two important aspects of the macaque prefrontal connectivity architecture. First, the predictions of the model were not confirmed and the wiring of the macaque prefrontal cortex does not comply with the proposed principles of the model. Second, while our results oppose to an asymmetry based hierarchical gradient oriented along the anterior-posterior axis, a complementary analysis aimed at a hierarchical arrangement of the prefrontal regions based on the laminar patterns of connectivity, i.e. the cortical layers constituting the origin and termination of the connections. Such analysis revealed that a laminar based hierarchy might be a more compatible organization principle suitable for models advocating an anterior-posterior gradient. Conjointly, our results indicate that the different concepts of hierarchy, i.e. asymmetry based and laminar based, capture distinct aspects of the organization of the connectivity of the prefrontal cortex of the macaque. These diverse hierarchical arrangements can be used to develop distinct and complementary hypotheses on the function of the prefrontal cortex.

The previous chapters examined the human and macaque brain by employing data from either species. Such approaches aim at understanding the architecture of the (pre)frontal cortex at the systems level. Additionally, they offer hints and insights into comparative aspects of the connectivity of the brain of the two species. However, the aforementioned approaches lack a direct rigorous comparison of the connectivity of the two species. Chapter 4 aimed at fulfilling such a need. We directly compared the connectivity patterns and topological properties of the brains of the two species. We adopted a quantitative whole brain approach for such comparison. Our results indicated that the wiring of the macaque and the human brain as a whole is significantly similar. A region wise analysis examining distinct aspects of connectivity preservation revealed interspecies similarities for the majority of the brain regions. Some discrepancies were also discernable primarily involving parietal and limbic cortical regions. We also uncovered a common structural backbone that appears preserved across primate 
evolution. Our study, through a quantitative interspecies analysis, offers a translational bridge between macaque and human research and illustrates evolutionary aspects of the connectivity of the primate brain. The results, while arguing for the suitability of the macaque brain as a model, also pinpoint some discrepancies that should be taken into account. Such discrepancies might reflect wiring features that are unique in each species.

In Chapter 5 we examined functional connectivity and task evoked responses of the human brain in a developmental context. Our analysis, illustrates that whole brain short and long range functional connectivity strength and task based activity constitutes information that can discriminate between age groups during adolescence. Moreover, the discriminative task based activity patterns do not appear to be bound to specific cognitive processes. Both functional connectivity and activity discriminative patterns are widespread across the whole brain, pinpointing to large scale changes during adolescence. Our approach allowed us to relate functional connectivity strength and task induced activation. The more pronounced functional connectivity strength differences were not confined to task induced discriminative regions. With the current estimation of functional connectivity strength maps, task induced and functional connectivity strength discriminative patterns seem to follow a segregated "maturation trajectory" during adolescence where different regions carry the most discriminative information. Our study illustrates the fruitfulness of advanced techniques like machine learning in order to uncover subtle functional changes of the maturing human brain.

\section{$\underline{\text { Future directions }}$}

The architecture of the brain is articulated in multiple levels. The complexity of the brain in conjunction with the vast amount of data produced from different labs around the world necessitates methodological and technological advancements that could unlock the rules pertaining to brain architecture.

A detailed mapping of the multilayered architecture of the brain calls for a multimodal approach (Toga et al., 2006). Advancements in neuroimaging protocols and high field MRI scanners (Heidemann et al., 2012) alongside with quantitative observerindependent tools (Schleicher et al., 1999), and advancements in data-mining methods (Lancichinetti et al., 2010) are promising for the generation of fine grained maps from data derived from different modalities and scales. Ideally, the resulting maps would be injected back to the neuroscientific community through neuroinformatics databases. Their incorporation into software suites will offer a means for an objective comparison of the maps derived from different datasets and modalities, as well as from different species (Van Essen and Dierker, 2007). Such directions will help enrich and finally even replace maps that have dominated neuroscience for over a century.

The recent blooming field of connectomics has already brought forth novel findings on brain structure and function. A future direction for comparative studies, valuable for clinical, systems and cognitive neuroscience, is to adopt a rigorous quantitative approach in analogy to other scientific disciplines, e.g. comparative genomics (Berg and Lässig, 2006), facing similar challenges with comparative neuroscience. Such a direction, which could be termed as comparative connectomics, will offer a valuable translational bridge between research in animals and research in humans. The quantitative nature of such approach will gradually unlock preserved and diverging features of the brain of mammals in general and primates in particular. Given the complexity of the problems faced by comparative studies, the detailed evolutionary 
trajectory of the multilayered architecture of the brain is highly unlikely to be uncovered solely with "traditional" observer dependent approaches. Since the connectome of each species is articulated at different levels (Sporns et al., 2005), e.g. macro and micro structures, long range interregional connections and intrinsic local microcircuitry, comparative studies should also compare the brain of two species at different levels. Hence, in accordance with the remarks for the need of multimodal atlases, interspecies comparisons should take place in different "resolution" levels (e.g. long range connectivity and intrinsic microcircuits) and micro-architecture levels (e.g. cyto- and receptoarchitecture). This will result in a high dimensional data space wherein the multilayered architecture of the brain can be studied from an evolutionary point of view. Certain studies indicate that while at a regional level intervention from dopaminergic projections do not differ between humans and macaques, at a laminar level certain organizational differences are discernable (Raghanti et al., 2008). This pinpoints that interspecies differences and similarities might co-exist and articulated at different levels. Hence, the aforementioned future direction in comparative studies is necessary for the development of more accurate heuristics and extrapolations of findings from animal research to humans and the development of models of the human brain.

Hopefully our own studies constitute a step towards the aforementioned future directions.

\section{$\underline{\text { Relevance to clinical applications }}$}

Advancements in fundamental neuroscience are necessary for the improvement of the treatment of mental disorders and clinical diagnosis.

The importance of imaging technologies is reflected for instance in deep brain stimulation treatment of depression (Ressler and Mayberg, 2007). Imaging techniques are important for the proper targeting of specific brain structures linked to pathologies. Given the extensive intersubject variability of the distinct cortical fields, a more accurate targeting, potentially resulting in more effective treatments, can benefit from the subject-wise in vivo parcellation techniques presented in this thesis. Such in vivo parcellation techniques might also appear to be helpful for pre-operative neurosurgery mapping. Task based imaging has been already employed for such purposes, e.g. for resection of brain tumours in the vicinity of the premotor cortex (Amiez et al., 2008). The lack of robust functional localizers for the frontal lobes might pose limitations of the usage of task based imaging techniques. Thus, our parcellation from resting state fMRI might constitute a complementary or alternative approach. The short acquisition time of the procedure, the effortless participation of the subjects and the lack of the need for their training constitute additional advantages of our approach.

Due to ethical and methodological issues the brains of animals serve as models for the human brain. Especially the macaque brain remains the dominant primate brain model (Passingham et al., 2009). By employing a quantitative approach, our studies might help develop more accurate models for the human brain and generate predictions about its function and possible alterations underlying brain pathologies. Our comparative approach offers a translational bridge between macaque and human research by indicating which regions have preserved their connectivity during evolution. Such preservation might entail a relative functional preservation and, at a gross level, the role of these regions, as revealed in macaque research, could be extrapolated to the human brain. Moreover, "network topology homologues", such as regions of the structural backbone, could be used for generating predictions for the human brain. For instance, 
lesions in the macaque brain and their impact on brain dynamics might be useful for developing heuristics for the impact of clinically depleted or lesioned homologous regions in the human brain.

Lastly, neuroimaging techniques along with machine learning methods have already started to be used for clinical diagnosis (e.g. Chang et al., 2012). Classification techniques based exclusively on phenotypic data seem to outperform imaging based ones (Brown et al., 2012). However, an imaging based approach for clinical diagnosis has the benefit of highlighting the neurobiology underlying certain pathologies. Machine learning techniques in combination with multimodal datasets can pinpoint to the most discriminative brain regions and features of brain organization (e.g. structure and connectivity) that are affected in particular pathologies. Our machine learning approach, as well as the adoption of both task based and connectivity information, can also be used in a clinical context.

\section{$\underline{\text { References }}$}

Amiez C, Kostopoulos P, Champod AS, Collins DL, Doyon J, Maestro R Del, Petrides M (2008) Preoperative functional magnetic resonance imaging assessment of higher-order cognitive function in patients undergoing surgery for brain tumors. J Neurosurgery 108:258-68.

Berg J., Lässig M. (2006) Cross-species analysis of biological networks. Proc Natl Acad Sci U S A 103:10969-72.

Brown MRG Sidhu GS, Greiner R, Asgarian N, Bastani M, Silverstone PH, Greenshaw AJ, Dursun SM (2012) ADHD-200 global competition: diagnosing ADH using personal characteristic data can outperform resting state fMRI measurements. Front Syst Neurosci 6:1-22.

Chang CW, Ho CC, Chen JH (2012) ADHD classification by a texture analysis of anatomical brain MRI data. Front Syst Neurosci 6:1-13.

Heidemann RM, Anwander A, Feiweier T, Knösche TR, Turner R (2012) k-space and q-space: Combining ultra-high spatial and angular resolution in diffusion imaging using ZOOPPA at 7 T. NeuroImage 60:967-978.

Passingham R (2009) How good is the macaque monkey model of the human brain? Curr Opin Neurobiol 19:6-11.

Raghanti MA, Stimpson CD, Marcinkiewicz JL, Erwin JM, Hof PR, Sherwood CC (2008) Cortical dopaminergic innervation among humans, chimpanzees and macaque monkeys: a comparative study. Neuroscience 155:203-220.

Ramasco J, Fortunato S, Lancichinetti A, Radicchi F (2011) Finding statistically significant communities in networks. PloS One 6:e18961.

Ressler KJ, Mayberg HS (2007) Targeting abnormal neural circuits in mood and anxiety disorders: from the laboratory to the clinic. Nat Neurosci 10:1116-1124.

Schleicher A, Amunts K, Geyer S, Morosan P, Zilles K (1999) Observer-independent method for microstructural parcellation of cerebral cortex: a quantitative approach to cytoarchitectonics. NeuroImage 177:165-177.

Sporns O, Tononi G, Kötter R (2005) The Human Connectome: a structural description of the human brain. PloS Comput Biol 1:e42.

Toga AW, Thompson PM, Mori S, Amunts K, Zilles K (2006) Towards multimodal atlases of the human brain. Nat Neurosci Rev 7:952-966. 


\section{Chapter 6}

Van Essen DC, Dierker DL (2007) Surface-based and probabilistic atlases of primate cerebral cortex. Neuron 56:209-25. 


\section{Samenvatting}

In dit proefschrift zijn de opbouw en de connectiviteit van het brein van de Makaak en van de mens onderzocht. Hiertoe is gebruikt gemaakt van in vivo beeldvorming van de menselijke hersenen, neuroanatomische data van de Makaak uit een neuroinformatica database en analysetechnieken uit de netwerkwiskunde.

Een eerste studie (hoofdstuk 2) onderzoekt het in vivo afbakenen van de corticale velden van de menselijke laterale frontale cortex (LFC) en het in kaart brengen van de onderlinge functionele connectiviteit. Uitgaande van functionele MRI data tijdens rust hebben we neuroanatomisch realistische "functionele modules" blootgelegd. Ze zijn realistisch omdat ze overeenstemmen met vermeende anatomische corticale substraten / corticale velden, beschreven in eerdere studies over de onderverdeling van de hersenschors op grond van hun microstructuur. De functionele verbindingen? van elk corticaal veld geeft informatie over de bijdrage van dit veld aan het LFC functioneren. De functionele connectiviteit van de gevonden corticale velden lijkt grotendeels overeen te komen met de anatomische connectiviteit van vermeende homologe corticale velden in de LFC van de Makaak. Onze benadering onthult een organisatieschema van corticale velden dat ten dele de gangbare modellen van de menselijke LFC bevestigt. De gangbare modellen zijn grotendeels ontwikkeld op basis van bevindingen uit onderzoek bij Makaken. Derhalve stellen onze resultaten een verbeterd onderverdelingschema voor van de menselijke LFC, dat als platform kan functioneren voor toekomstige taakgebaseerde / cognitieve fMRI studies bij de mens, en leidraad voor LFC modellen, en dat bovendien het onderzoek in het menselijk brein en het Makaak brein verbindt.

Onze volgende studie gaat dieper in op de principes achter de verbindingen in de (pre)frontale hersenschors. In Hoofdstuk 3 testen we expliciet de voorspellingen van een invloedrijk model dat in de organisatie van de prefrontale cortex een hiërarchische anterieure-posterieure gradiënt veronderstelt. Daarbij wordt hiërarchie opgevat als assymmetrie in de verbindingen tussen corticale velden. Onze formele netwerkbenadering, uitgaande van analysettechnieken die eerder al werden gebruikt in systeembiologie, bracht twee belangrijke aspecten aan het licht over de opbouw van prefrontale verbindingen in de Makaak. Ten eerste werden de predicties van het model niet bevestigd: de gekende verbinden van de prefrontale cortex van de Makaak sluiten niet aan bij de voorgestelde principes van het model. Ten tweede toonde een een aanvullende analyse dat met een andere definitie van hiërarchische verbindingen wel een meer plausibel organisatiemodel naar voor komt, waarin een anterieure-posterieure gradiënt zichtbaar is. Deze andere definitie van hiërarchie is gebaseerd op de laminaire patronen van connectiviteit - i.c. de distributie over de corticale lagen van de oorsprong en het einde van de connecties. Samen suggereren onze resultaten dat de verschillende hiërarchische concepten, in dit geval asymmetrisch gebaseerd en laminair gebaseerd, verschillende aspecten van de organisatie van de verbindingen van de prefrontale cortex van de Makaak omvatten. Deze diverse hiërarchische rangschikkingen kunnen gebruikt worden om verschillende afzonderlijke en complementaire hypotheses te ontwikkelen betreffende de functie van de prefrontale cortex.

In de voorgaande hoofdstukken werd ofwel het menselijk brain, ofwel het Makaak brein onderzocht. Dergelijke soortspeciefieke benaderingen zijn gericht op het begrijpen van de neurale architectuur van de prefrontale cortex op systeemniveau. Tevens bieden zij aanwijzingen over vergelijkbare aspecten van die neurale architectuur in beide soorten. Er ontbreekt echter een directe vergelijking van de hersenorganisatie in beide 
soorten. Hoofdstuk 4 komt tegemoet aan deze behoefte. In dit hoofdstuk worden de patronen van connectiviteit en de topologische eigenschappen van het brein van beide soorten direct vergeleken, en dit op een kwantitatieve wijze en over het gehele brein. Onze resultaten tonen dat de neurale verbindingen van de Makaak en de mens, als geheel, significant homoniem zijn. Een analyse per corticaal veld, waarbij afzonderlijke aspecten van connectiviteitsbehoud werden onderzocht, toonde soortovereenkomsten voor de meerderheid van de velden. Daarnaast werden ook enkele markante discrepanties vastgesteld, voornamelijk met betrekking to pariëtale en limbische hersenregio's. We hebben eveneens een gemeenschappelijke structurele ruggengraat bloot gelegd welke behouden lijkt te zijn gebleven gedurende de evolutie van de primaat. Door het gebruik van kwantitatieve vergelijkende analyse vormt onze studie een translationele brug tussen onderzoek in de Makaak en de mens, en belicht ze evolutionaire aspecten van de connectiviteit van het brein van primaten. Hoewel de resultaten de geschiktheid van het brein van de Makaak als model voor de menselijke hersenen bevestigen, tonen zij ook enkele discrepanties waar rekening mee gehouden dient te worden. Dergelijke discrepanties reflecteren mogelijk verbindingskenmerken die uniek zijn in elke soort.

In een laatste studie (hoofdstuk 5) hebben we functionele connectiviteit en taakgerelateerde hersenactiviteit in het menselijk brein onderzocht in de context van ontwikkeling. Onze analyse toonde dat de sterkte van kortreikende en verreikende functionele connecties, alsook van taak gerelateerde activiteit, informatie bieden waarmee onderscheid gemaakt kan worden tussen leeftijdsgroepen gedurende de adolescentie. Bovendien bleken de onderscheidende taak-gebaseerde activiteitspatronen niet gebonden te zijn aan specifieke cognitieve processen. De onderscheidende patronen van zowel functionele connectiviteit als taak-gerelateerde activiteit waren wijdverbreid in het hele brein. Ze wijzen op veranderingen op grote schaal tijdens de adolescentie. Onze benadering maakte het mogelijk om de mate van verandering in functionele connectiviteit en in taak-geinduceerde activatie aan elkaar te relateren. We stelden vast dat de paronen van met de leeftijd gewijzigde connectiviteit en taak-gerelateerde activatie maar beperkte overeebsomst vertonen. Op grond van de gevonden resultaten moeten we besluiten dat functionele connectiviteit en takactivatie een onderscheiden "ontwikkelings-traject" volgen gedurende de adolescentie, waarbij wijzigingen in beide domeinen zich over verspreide gebieden in de hersenen voltrekken. Ons onderzoek illustreert hoe vruchtbaar geavanceerde technieken zoals multivariate patroon analyse kunnen zijn bij het blootleggen van subtiele functionele veranderingen in het volwassen wordende menselijk brein. 


\section{Acknowledgements}

And now for something different, here it is - the forced memoire that has to be done! Well, there is no commemoration of anything in here compared to truly commemorating acts. In the future, that is our times, everyone can be world-famous for 15 minutes well the thesis acknowledgements exist as if to only make everyone a writer for 15 minutes! So I have to grow my beard, light my pipe, and start filling some lines with snapshots of the past and glimpses of the future.

It all started on a sunny afternoon in the land of the demigods when the summer craziness was not enough to soothe some naïve expectations and dreams. The trajectory that they were shaping was primarily fuelled with uneasiness for the "needs of the market", the options running low, the shrieks of the horizon and a wanderer's needs? Maybe it is luck, maybe it can be considered a trademark of our spoiled generation, but some wishful thinking transformed into a concrete plan with the ever present support of my parents - my sister approved as well and now she better understands why I had these tendencies for escaping back then.

So here we are, after some cigarettes at the train station I am walking the streets of a new city, I cross the street where in some months I would be anxiously waiting. Rain, monolithic churches, and a well respected academic environment - all of these looked impressive and promising while I was sitting in the bar where I would meet Ardi, Anna, Vaso, Adrien, random funny guys and newcomers that made the place thrive with fresh perspectives. The Old Europe, the north - back then it could sing a charming song, but the technocrats also inhabit places of idealistic paraphernalia. After one year of work and fun, uncertainty emerged. The voice on the phone says: "The position is yours $\mathrm{Mr}$ Goulas". Delighted to hear from you dr. Stiers. And delighted to be in a new working environment. Frost covers everything on my way here and a sad thought is interrupted by the warm welcoming from my officemates and colleagues. Afterwards I am among strangers around a table. A professor looks like a bust of Zeus. A guy is making jokes and witty remarks about neuroscientific work. An underlying sarcasm wraps everything he utters and he has a penetrating way of thinking. He is one of my promotors. After some weeks we have big plans with Peter. I enjoy freedom and creativity and imagination substitutes the flaws caused by my terrible math teacher. We meet a tall professor, with an imposing appearance and discuss some new ideas. "This is professor Uylings" Peter says. I leave the café at the train station with the thought that with him on our side our work will become classier! Some years afterwards there is some proof that this wishful thinking came true.

I devour the summer holidays and a militant spirit keeps me vigilant. I have to find "enemies", something to surpass, in order to stay awake and ambitious. A vacuum opens and stays there for some time, autumn paves the way for a wasteland. No worries - let the good times roll in...

I touch vinyls and cold glasses of beer, spend time in Utrecht and Amsterdam, catch up with friends at the ramblas of Barcelona. "Here is the cake that I baked. Wanna try?" I have a new neighbour and after a while yet another one. Fortunately - the other guy that was fighting with his girlfriend and listening to de Alla music on Sunday mornings is gone! I go to Zondag and then Take 5. After a while I leave the place in frustration. Joao and the guys suggest going to this party. Well, our plans coincide - let's check it out! Kamil also joins. Sunny BBQs, and long days with cocktails, flip-flops and chats are revitalizing. Some people have to do their laundry and do not join. Mehrdad is 
showing up late as always - "Whaaat's up guyyyss!?!?" Lars is so freaking good at table tennis that it makes me angry during our sporty summer breaks. Ok, never mind we are off to Matteo's and Roberta's restaurant where gourmet dishes are piling up. The bell rings and Marin shows up. But where is Aline? Damn! She's already gone by now, so no after dinner partyyyyyy. Martin likes the squat place so we are frequent visitors there. Time is passing fast and the resume does not grow a lot. I receive the comments for our work and they make me want to land a fast combination a la Yuriorkis Gamboa style on everyone. But it is a learning process - in an imperfect world I should have expected this. Technicalities won't suffice - I need more visual propaganda. After some months Peter enters with a triumphant smile in my office. I take the rest of the day off to sunbath with mojitos. I am hungry again for more scientific stuff. Nikos and Philippos complain about my Skype being out of use. Well, guys after all these years the communication lines are always present and distances cannot (etc etc all these cheesy stuff). Rosanne rushes in, full of intellectual seizures, stating this, stating that. Cerebral overdose reigns and I say: "Truthfully, you are a masterpiece!". Here is a plan that can be executed with Matteo. I run upstairs and after a while we have a deal! I go home and check the blog. Mitsos has uploaded new rips - after all this vinyl re-issue company idea becomes more and more appealing.

Here it comes again, this urge for change. Things are dynamic by their nature, so this is not an awkward feeling. We follow every possible promising event with Joao et al. and sometimes some lyrics of Iggy Pop flash in my mind. We drink some cognac with Lars and Britta and we hack Lars' brain. Popi is bikeless and too far away to join. Summer kicks in and introductions take place. It feels like I already know a person and pretty well. After a while a gate to dreamworlds appears and I am sucked in. Sometimes I speak like Count Dracula.

Business travels enhance my interest in my scientific work. There are many gaps in this field that excite me. Back to the foundations again - this results in one project and an over the top sketch of a task to be undertaken the forthcoming years. But then again...Thoughts, plans, wishes... Here is one more: May the owl of Minerva transform to the eagle of Apollo. And we might be watching this transformation from far away. 


\section{Curriculum Vitae}

Alexandros Goulas was born in Athens, Greece on the $3^{\text {rd }}$ of May 1982. After his highschool studies he enrolled at the Technological Educational Institute of Athens to study Informatics. Alexandros finished his bachelor degree in 2007 and moved to Leuven, Belgium for his master studies in Artificial Intelligence and Cognitive Science. He started his Phd at the Department of Neuropsychology and Psychopharmacology at Maastricht University in January 2009 under the supervision of dr. Peter Stiers. Currently he is working as a post-doc researcher at the same department. 


\section{Publications and Presentations}

\section{Publications}

Goulas A, Uylings HBM, Stiers P (2012) Unravelling the intrinsic functional organization of the human lateral prefrontal cortex: a parcellation scheme based on resting-state fMRI. Journal of Neuroscience 32:10238 -10252.

Goulas A, Uylings HBM, Stiers P (in press) Mapping the hierarchical layout of the structural network of the macaque prefrontal cortex. Cerebral Cortex.

Keulers EHH, Goulas A, Jolles J, Stiers P (2012) Maturation of task-induced brain activation and long range functional connectivity in adolescence revealed by multivariate pattern classification. Neuroimage 60:1250-1265.

\section{Presentations}

\section{Posters}

Goulas A, Stiers P Parcellation of the human lateral frontal cortex from resting-state fMRI. Presented at OHBM 2011.

Goulas A, Uylings HBM, Stiers P Mapping the dominant direction of the structural network of the macaque prefrontal cortex reveals its hierarchical structure. Presented at FENS 2012.

Stiers P, Goulas A., Samara Z, Uylings HBM Intrinsic functional connectivity parcellation and task activation in human lateral frontal cortex. Presented at the $3^{\text {rd }}$ Biennial Conference on Resting State Brain Connectivity 2012 Magdeburg.

Goulas A, Bastiani M, Bezgin G, Roebroeck A, Stiers P Comparative quantitative analysis reveals preserved structural connectivity patterns in the macaque and human brain. Presented at the Society for Neuroscience meeting 2012 New Orleans.

Stiers P, Goulas A, Uylings HBM Task-induced brain activation in relation to intrinsic functional connectivity parcellation of the human lateral prefrontal cortex. Presented at the Society for Neuroscience meeting 2012 New Orleans.

\section{Invited talks}

Mapping the architecture of the prefrontal cortex in human and non-human primates, May 2012, Max Planck Institute for Human Cognitive and Brain Sciences, Leipzig. Germany

Mapping brain networks: cortical fields and connectivity, February 2013, Attention control in neural circuits laboratory, York University, Toronto, Canada 
